\title{
Bildungsverläufe von der
}

Einschulung bis in den

ersten Arbeitsmarkt

Theoretische Ansätze, empirische Befunde und Beispiele 
Bildungsverläufe von der Einschulung bis in den ersten Arbeitsmarkt 
Markus P. Neuenschwander · Christof Nägele (Hrsg.)

\section{Bildungsverläufe von der Einschulung bis in den ersten Arbeitsmarkt}

Theoretische Ansätze, empirische Befunde und Beispiele 
Herausgeber

Markus P. Neuenschwander

Solothurn, Schweiz
Christof Nägele

Solothurn, Schweiz

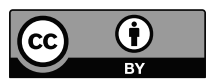

ISBN 978-3-658-16980-0

ISBN 978-3-658-16981-7 (eBook)

DOI 10.1007/978-3-658-16981-7

Die Deutsche Nationalbibliothek verzeichnet diese Publikation in der Deutschen Nationalbibliografie; detaillierte bibliografische Daten sind im Internet über http://dnb.d-nb.de abrufbar.

\section{Springer VS}

(C) Der/die Herausgeber bzw. der/die Autor(en) 2017. Dieses Buch ist eine Open-Access-Publikation. Open Access Dieses Buch wird unter der Creative Commons Namensnennung 4.0 International Lizenz (http://creativecommons.org/licenses/by/4.0/deed.de) veröffentlicht, welche die Nutzung, Vervielfältigung, Bearbeitung, Verbreitung und Wiedergabe in jeglichem Medium und Format erlaubt, sofern Sie den/die ursprünglichen Autor(en) und die Quelle ordnungsgemäß nennen, einen Link zur Creative Commons Lizenz beifügen und angeben, ob Änderungen vorgenommen wurden.

Die in diesem Buch enthaltenen Bilder und sonstiges Drittmaterial unterliegen ebenfalls der genannten Creative Commons Lizenz, sofern sich aus der Abbildungslegende nichts anderes ergibt. Sofern das betreffende Material nicht unter der genannten Creative Commons Lizenz steht und die betreffende Handlung nicht nach gesetzlichen Vorschriften erlaubt ist, ist für die oben aufgeführten Weiterverwendungen des Materials die Einwilligung des jeweiligen Rechteinhabers einzuholen.

Die Wiedergabe von Gebrauchsnamen, Handelsnamen, Warenbezeichnungen usw. in diesem Werk berechtigt auch ohne besondere Kennzeichnung nicht zu der Annahme, dass solche Namen im Sinne der Warenzeichen- und Markenschutz-Gesetzgebung als frei zu betrachten wären und daher von jedermann benutzt werden dürften.

Der Verlag, die Autoren und die Herausgeber gehen davon aus, dass die Angaben und Informationen in diesem Werk zum Zeitpunkt der Veröffentlichung vollständig und korrekt sind. Weder der Verlag noch die Autoren oder die Herausgeber übernehmen, ausdrücklich oder implizit, Gewähr für den Inhalt des Werkes, etwaige Fehler oder Äußerungen. Der Verlag bleibt im Hinblick auf geografische Zuordnungen und Gebietsbezeichnungen in veröffentlichten Karten und Institutionsadressen neutral.

Gedruckt auf säurefreiem und chlorfrei gebleichtem Papier

Springer VS ist Teil von Springer Nature

Die eingetragene Gesellschaft ist Springer Fachmedien Wiesbaden GmbH

Die Anschrift der Gesellschaft ist: Abraham-Lincoln-Str. 46, 65189 Wiesbaden, Germany 


\section{Vorwort}

Kinder und Jugendliche werden über mehrere Schuljahre Schritt für Schritt auf die Berufsausbildung, die Erwerbstätigkeit und das lebenslange Lernen vorbereitet. Allerdings profitieren sie je nach Bildungsverlauf unterschiedlich viel von den schulischen Angeboten. Was Schülerinnen und Schüler lernen, hängt nicht nur von der Qualität des Unterrichts ab, sondern auch ganz wesentlich von der Schulkarriere, die sie durchlaufen. So verläuft zum Beispiel die Leistungsentwicklung über die Schuljahre nicht gradlinig und linear. Wenn sie ein Schuljahr wiederholen oder in Schulniveaus mit geringen Anforderungen zugewiesen werden, ist ihr Lernfortschritt geringer. Entscheidend ist, ob die Schule die Schülerinnen und Schüler in ein passendes schulisches Umfeld zuweist.

Bildungsverläufe werden im Kindergarten vorbereitet und während der obligatorischen Schulzeit, Berufsausbildung und in der Weiterbildung fortgesetzt. Dabei bedingen und beeinflussen sich individuelle, institutionelle und soziale Faktoren. So hängen Bildungsverläufe von den Leistungen, der Motivation und dem Verhalten der Schülerinnen und Schüler ab. Sie werden aber auch - auf gesellschaftlicher Ebene durch das Bildungssystem gesteuert (Selektion und Durchlässigkeit). Wichtig sind soziale Faktoren: Die Eltern und Lehrpersonen beeinflussen als Vorbilder und durch ihre Unterstützung und Förderung und vor allem auch durch ihre Erwartungen die Lernprozesse, Selektionsentscheidungen und somit die Bildungsverläufe der Jugendlichen wesentlich.

Dieses Buch wurde aufgrund des Erfolgs einer wissenschaftlichen Tagung mit dem Titel „Erfolgsfaktoren und Risikosituationen in Bildungsverläufen“, an der viele Expertinnen und Experten aus der Schweiz und Deutschland im Januar 2016 teilgenommen hatten, initiiert. Das hohe Interesse an der Tagung und deren positive Evaluation belegen die hohe Aktualität. Allerdings wurden die Tagungsbeiträge für die geplante Publikation gründlich überarbeitet, so dass hiermit nicht "nur“ ein Tagungsbuch vorliegt, sondern eine eigenständige Darstellung der Thematik. 
Das vorliegende Buch umfasst theoretische und empirische Originalbeiträge aus längsschnittlich angelegten Forschungsprojekten in der Schweiz, die für die Bildungsöffentlichkeit relevant und neuartig sind. Das Buch ist in vielerlei Hinsicht originell: Es vereint übersichtlich Artikel zu ähnlichen Themen, so dass direkte Vergleiche zwischen den Beiträgen möglich sind und ein grosser Fundus an Wissen kompakt und attraktiv präsentiert wird. Es verbindet systematische Theoriearbeit mit Befunden aus grossen Längsschnittstudien. Es schliesst neben der Schule auch die Berufsbildung auf der Sekundarstufe II ein. Es ermöglicht dadurch, Gemeinsamkeiten und Unterschiede, Kontinuitäten und Diskontinuitäten zwischen der obligatorischen Schule und der Berufsbildung aufzuzeigen. Die Einzelbeiträge gerade in der Zusammenschau führen die wissenschaftliche Diskussion zur Thematik weiter. Zudem resultiert eine verdichtete Darstellung von Ergebnissen, die nicht nur für die Bildungs-und Berufsbildungsforschung wichtig ist, sondern auch für die Bildungsadministration, die Berufsberatung und die Praktikerinnen und Praktiker in Schule und Berufsbildung.

Das Buch beginnt mit zwei Theorieartikeln, welche konzeptuelle Grundlagen für Schulübergänge, und den Übergang von der Sekundarstufe I in die berufliche Grundbildung liefern. Diese Modelle bilden theoretische Folien, auf der die nachfolgenden empirischen Beiträge interpretiert werden können. Danach werden diachrone Bildungsverläufe in der Schule thematisiert. Gérard Bless verweist in seinem Beitrag zu irregulären Schullaufbahnen im Rahmen der obligatorischen Schule und zur Klassenwiederholung eindrücklich auf die negativen Folgen der Selektion und deren „Nebenwirkungen“ sowie auf die Gefahr, dass oberflächliche, auf Individualdaten des Bundesamts für Statistik basierende Analysen dazu führen können, diese Problematik zu verharmlosen. Urs Moser, Jeannette Oostlander und Martin J. Tomasik beleuchten in ihrem Beitrag zu sozialen Ungleichheiten im Leistungszuwachs und bei Bildungsübergängen den Informationsvorteil, den Längsschnittstudien bieten. Sie beschreiben längsschnittlich den Leistungszuwachs während der Schule, in Abhängigkeit nicht nur der Schulleistungen, sondern auch der sozialen Herkunft und Erstsprache. Sie folgern, dass Programme zum Nachteilsausgleich möglichst früh in der schulischen Laufbahn der Kinder ansetzen müssen.

In einem zweiten Teil werden Übergänge und deren Vorbereitung in der Schule thematisiert. Sara Benini, Jennifer C. Fräulin und Markus P. Neuenschwander stellen in ihrem Beitrag den FOKUS-Ansatz zum Umgang mit hyperaktiven, impulsiven und unaufmerksamen Kindern auf der Schuleingangsstufe vor. Der FOKUS-Ansatz verfolgt das Ziel, Lehrpersonen für die Problematik der Unaufmerksamkeit und Hyperaktivität/Impulsivität zu sensibilisieren und sie weiterzubilden, so dass die Nachteile dieser Kinder in ihren Bildungsverläufen frühzeitig reduziert werden (Prävention). 
Stephan Rösselet und Markus P. Neuenschwander gehen in ihrem Beitrag zur Akzeptanz und Ablehnung von Schülerinnen und Schülern beim Übertritt in die Sekundarstufe I auf eine in der Forschung oft vernachlässigte Herausforderung ein. Sie beleuchten die Veränderung des Klassenstatus (Soziogramm) am Beispiel der Akzeptanz und Ablehnung beim Übergang in die Sekundarstufe I und zeigen Bezugsgruppeneffekte. Diese führen dazu, dass sich mit dem Übertritt in ein Schulniveau mit hohen Ansprüchen der Klassenstatus verschlechtert.

Im Beitrag von Markus P. Neuenschwander und Edith Niederbacher zu Schulniveau- und Leistungserwartungen von Lehrpersonen und der Leistungsentwicklung beim Übergang in die Sekundarstufe I wird sehr deutlich dargelegt, welch grossen Einfluss die Erwartungen der Lehrpersonen an die Leistungsentwicklung einer Schülerin oder eines Schülers auf die Beurteilung deren oder dessen Leistung hat. Der originäre Aspekt dieses Beitrags ist, dass die Wirkung impliziter (nicht direkt kommunizierter) und expliziter Erwartungen der Lehrpersonen auf die Leistungsentwicklung beim Verbleib in einer Klasse im Vergleich zur Situation eines Schulübertritts gezeigt wird.

Der Beitrag von Markus P. Neuenschwander zu Anpassungsprozessen beim Übergang in die Sekundarstufe I diskutiert die Abnahme der wahrgenommenen Passung einer Schülerin oder eines Schülers zur Schule beim Übertritt in die Sekundarstufe I. Er zeigt, dass sich nach Kontrolle des Alters die Passungswahrnehmung, aber auch die Motivation und die Leistungen, in Deutsch und Mathematik, beim Eintritt in die Sekundarstufe I deutlicher verschlechtern als beim Verbleib in der Primarschule. Obwohl die Schulniveaus in der Sekundarstufe I höhere Leistungshomogenität schaffen, entwickelt sich die Passung nach Eintritt in die Sekundarstufe I ungünstig.

Im dritten Teil werden Übergänge in eine Ausbildung auf der Sekundarstufe II und der Tertiärstufe diskutiert. Albert Düggeli beschreibt die Situation von Schülerinnen und Schülern in einem tieferen Schulniveau, die einen direkten Einstieg in die Lehre geplant hatten, diesen aber nicht realisieren konnten. Es ist die Spannung zwischen Aspiration und Realisierung im letzten obligatorischen Schuljahr. Es wird diskutiert, wie die Zuweisung zu einem bestimmten - in diesem Fall tiefen - Schulniveau die individuellen Aspirationen konterkariert. So sind die Kriterien, die einen direkten Übertritt in eine zertifizierende Ausbildung auf der Sekundarstufe II ermöglichen, je nach Schulniveau differenziert. Dies beeinflusst das Verhalten und die Aktivität bezüglich der Suche einer Anschlusslösung

Im Beitrag von Christof Nägele, Simone Frey und Markus P. Neuenschwander wird die Passung zum Beruf, die berufliche Zufriedenheit, die Verbundenheit und die Wahl einer weiteren Aus- oder Weiterbildung thematisiert. Es zeigt sich, dass teil-objektive Masse der Passung, basierend auf der Erfassung beruflicher 
Interessen, weder die Zufriedenheit und Verbundenheit mit dem Beruf noch die weitere Ausbildung vorhersagen können. Ausschlaggebend ist hingegen die wahrgenommene Passung.

Verdienstvollerweise hat Sandra Hupka in einem abschliessenden Kapitel eine Übersicht mit Schlussfolgerungen für Forschung und Praxis formuliert. In einer Gesamtperspektive benennt sie wichtige Punkte aus den einzelnen Beiträgen und ergänzt Fehlendes. Insbesondere ergänzt sie bildungssoziologische Konzepte, die in der früheren Forschung immer wieder gewinnbringend in die Thematik eingeflossen sind.

Wir hoffen, mit diesen Beiträgen die Diskussion um Bildungsverläufe in wichtigen Aspekten zu befruchten. Auf dieser Grundlage können Hinweise abgeleitet werden, wie Kinder, Jugendliche und junge Erwachsene in ihren Bildungsverläufen unterstützt, soziale Ungleichheit abgebaut und Bildungsprozesse optimiert werden können.

Unser Dank gilt zuerst den Autorinnen und Autoren, die mit substanziellen Beiträgen zur Realisierung dieses Buchprojekts beigetragen haben. Wir danken der Pädagogischen Hochschule der Fachhochschule Nordwestschweiz für den finanziellen Beitrag, so dass das Buch für alle interessierten Personen ohne Kosten (open access) zugänglich ist. Wir danken dem Springer Verlag für die Unterstützung bei der Publikation. Wir würden uns freuen, wenn diese Publikation zu einer fruchtbaren Diskussion von Bildungsverläufen von der Einschulung in die Erwerbstätigkeit beitragen kann.

Solothurn, 28.2.2017

Die Herausgeber:

Markus P. Neuenschwander

Christof Nägele 


\section{Inhalt}

\section{A Theoretische Einbettung}

Schultransitionen - Ein Arbeitsmodell $\ldots . \ldots \ldots \ldots \ldots \ldots \ldots \ldots \ldots \ldots$ Markus P. Neuenschwander

Übergänge in die Berufsbildung - Ein Arbeitsmodell $\ldots \ldots \ldots \ldots \ldots \ldots . \ldots 21$ Christof Nägele und Barbara E. Stalder

\section{B Diachrone Verläufe in der Schule}

Irreguläre Schullaufbahnen im Rahmen der obligatorischen Schule und Forschungsergebnisse zur Klassenwiederholung ............... 39 Gérard Bless

Soziale Ungleichheiten im Leistungszuwachs und bei Bildungsübergängen . . . 59 Urs Moser, Jeannette Oostlander und Martin J. Tomasik

\section{C Übergänge in der Schule}

Der FOKUS-Ansatz: Hyperaktive, impulsive und unaufmerksame Kinder nach der Einschulung fördern $\ldots \ldots \ldots \ldots \ldots \ldots \ldots \ldots \ldots . \ldots \ldots$ Sara Benini, Jennifer C. Fräulin und Markus P. Neuenschwander

Akzeptanz und Ablehnung beim Übertritt in die Sekundarstufe I . . . . . . 103 Stephan Rösselet und Markus P. Neuenschwander 
Schulniveau- und Leistungserwartungen von Lehrpersonen und Leistungsentwicklung beim Übergang in die Sekundarstufe I . . Markus P. Neuenschwander \& Edith Niederbacher

Anpassungsprozesse beim Übergang in die Sekundarstufe I 143 Markus P. Neuenschwander

\section{D Übergänge in der Berufsbildung}

Direkter oder verzögerter Übertritt? Das letzte Schuljahr zwischen Aspiration und Realisierung ............................... 163 Albert Düggeli

Passung zum Beruf und die Wahl einer Aus- oder Weiterbildung $\ldots . \ldots . .181$ Christof Nägele, Simone Frey und Markus P. Neuenschwander

\section{E Ausblick}

Hoffen auf Passung? Homogenitätsfiktion und ihre Folgen $\ldots \ldots \ldots \ldots . . .201$ Sandra Hupka-Brunner 
A

Theoretische Einbettung 


\section{Schultransitionen - Ein Arbeitsmodell}

Markus P. Neuenschwander

\section{Zusammenfassung}

Die erziehungswissenschaftliche Forschung zu Schultransitionen hat wachsende Aufmerksamkeit und Bedeutung erlangt. Um vorliegende Konzepte und Befunde zu systematisieren und neue Forschungsfragen $\mathrm{zu}$ generieren, wird ein neues Arbeitsmodell zur Strukturierung von Schultransitionen vorgeschlagen. Es postuliert, dass Jugendliche gestützt auf ihre Fähigkeiten und askriptiven Merkmale Überzeugungen entwickeln, die zu Bildungsentscheidungen und Schulübergängen führen. Jugendliche passen sich nach dem Übertritt an den neuen Kontext an. Dieser Prozess ist in das Bildungssystem eingebettet und wird von den Eltern begleitet. Bildungsverläufe führen in die Berufsbildung bzw. das Studium und in die Erwerbstätigkeit. Sie werden in Weiterbildungen fortgesetzt. Der Nutzen dieses neuen Modells liegt in der Fokussierung auf Erklärungskonzepte, die das Verständnis von Übertritten und Bildungsverläufen vertiefen, neue Forschung ermöglichen und eine zielgerichtete Begleitung von Jugendlichen bei Übertritten ermöglichen.

\section{Schlagworte}

Transition, Sekundarstufe, Schule, Familie, Berufsbildung 


\section{$1 \quad$ Einleitung und Fragestellung}

In den letzten Jahren erhielt die Transitionsforschung steigende Aufmerksamkeit und etablierte sich allmählich zu einem eigenen interdisziplinär ausgerichteten Forschungszweig. In Weiterführung zu Bronfenbrenners (1981) Konzept des ökologischen Übertritts rückte die Perspektive in den Vordergrund, dass Lern- und Entwicklungsprozesse in Kontexte eingebunden sind (Lerner, 1999) und dass während des Übertritts von einem Kontext in einen anderen Lern- und Entwicklungsprozesse beschleunigt ablaufen. Übertritte treten mit dem Eintritt in eine Kinderspielgruppe, Kindertagesstätte, Kindergarten oder Schule bereits im Vorschulbereich auf. Danach folgen normative Übertritte in die Sekundarstufe I, in die Sekundarstufe II, in die Tertiärstufe bzw. in die Erwerbstätigkeit. Zudem treten non-normative Übergänge beispielsweise beim Wiederholen oder Überspringen einer Klasse, bei Zuweisungen in die Sonderschulung oder bei Schul- oder Lehrabbruch in der Sekundarstufe II auf. Es wird zwischen Schulübertritten, d.h. dem Übertritt von einer Schule in eine andere Schule (z. B. der Übertritt in die Sekundarstufe I oder in das Gymnasium), und dem Übertritt Schule-Beruf (zum Beispiel Übertritt von der Sekundarstufe I in die Berufsbildung) unterschieden. Die vorliegende Analyse konzentriert sich auf den Übertritt in die Sekundarstufe I und den Übertritt in die Sekundarstufe II (allgemeinbildend und berufsbildend).

Im Folgenden wird ausgehend von der einschlägigen Forschung ein Arbeitsmodell präsentiert, das Forschung systematisiert und Bedingungen und Ergebnisse von Übertritten in die Sekundarstufe I und II erklärt. Theoretische Modelle von solchen Übertritten fehlen bisher. Das Modell liefert eine Antwort auf die Frage, wie die Bedingungen und die Ergebnisse von Übertritten von den verschiedenen Akteuren gesteuert werden. Es schliesst Prozesse vor und nach dem Übertritt ein. Es soll Forschung strukturieren, aber auch Grundlagen für Interventionen liefern, wie Jugendliche in ihren Bildungsverläufen in die Berufsbildung oder das Studium unterstützt werden können.

\section{Leitideen}

Im ersten Schritt werden die Grundlagen dargestellt, auf denen das Arbeitsmodell basiert. 


\subsection{Lernen und Entwicklung im Kontext}

Mit Lerner, Theokas \& Jelicic (2005) wird davon ausgegangen, dass Jugendliche in verschiedene soziale Kontexte eingebettet sind, in denen sie lernen und sich entwickeln (Lave \& Wenger, 1991). Der Kontext umfasst verschiedene Personen, die aufgrund gemeinsamer, konstituierender Interessen über längere Zeit miteinander interagieren. Der Begriff Kontext meint sowohl das unmittelbare Umfeld (zum Beispiel die Schulklasse, die Familie) als auch das weitere Umfeld (zum Beispiel das Bildungssystem). Der Bildungskontext bezieht sich auf diejenigen Kontexte, in welchen geplant Lern- und Entwicklungsprozesse von Jugendlichen arrangiert werden. Der Person-in-Kontext-Ansatz impliziert zum Beispiel, dass Jugendliche in Schulklassen integriert sind, in welchen sie schrittweise unter Anleitung von Lehrpersonen neues Wissen erarbeiten (lernen) und sich in Auseinandersetzung mit Aufgaben, welche das Bildungssystem vorgibt, entwickeln (Eccles \& Roeser, 2011a).

Das Bildungssystem schafft Strukturen und Normen, mit denen sich Jugendliche auseinandersetzen und dadurch eigene Positionen und Überzeugungen erarbeiten (Sozialisation; Heinz, 2000). Die Art der Auseinandersetzung ist vielfältig: Jugendliche reflektieren beispielsweise Erwartungen und Werte von Bezugspersonen (Eccles \& Roser, 2011b), vergleichen sich mit Gleichaltrigen im gleichen Kontext (z. B. Bezugsgruppeneffekte, Marsh, 2005) und erwerben gemeinsam und kooperativ neues Wissen (z. B. Reinmann-Rothmeier \& Mandl, 2002).

Mit zunehmendem Alter vollziehen Jugendliche immer neue Schultransitionen. Damit entstehen Bildungsverläufe, die zu entsprechenden Bildungsabschlüssen führen und zu beruflichen Tätigkeiten qualifizieren. Bildungsverläufe resultieren also aus den Lern- und Entwicklungsprozessen in den jeweiligen Bildungskontexten und aus dem Übertritt in neue Bildungskontexte.

\subsection{Normen}

Bedingungen und Ergebnisse von Übertritten, aber auch Bildungsabschlüsse werden aufgrund sozialer Normen, die in Gruppen oder Gesellschaften gelten, bewertet. Normen sind gemeinsam geteilte Werte von Gruppen oder Gesellschaften. Normen bilden Orientierungshilfen für die Individuen bei der Planung und Gestaltung von Bildungsverläufen. Sie sind eine Grundlage für die Bewertung der Entscheidungen bzw. des Erfolgs von Übertritten. Normen leiten zudem Interventionen zur Gestaltung von Übertritten bzw. Selektionsprozessen. Allerdings werden in postmodernen Gesellschaften sehr unterschiedliche Normen vertreten (z.B. Welsch, 1988). Im Folgenden werden Normen genannt, welche aus verschiedenen 
Perspektiven für die Steuerung von Bildungsverläufen und bei der Bewertung von Übertritten diskutiert werden.

- Die Status Norm besagt, dass Jugendliche möglichst statushohe Abschlüsse erreichen sollen. D.h. , Ausbildungen sind attraktiv, die zu statushohen Berufen führen. Damit ist die Hoffnung auf eine interessante, verantwortungsvolle berufliche Tätigkeit verbunden, die öffentlichen Status und hohes Einkommen mit sich bringt. Beispielsweise ist die Attraktivität des Gymnasiums im Vergleich zur Berufsbildung für manche Eltern in der Statusnorm begründet. Es wird angenommen, dass mit dem gymnasialen Weg ein höherer Status einhergeht als mit einem berufsbildenden Weg.

- Manche staatlichen Organisationen vertreten eine Schwellennorm: Junge Menschen sollen mindestens einen Bildungsabschluss erreichen, mit dem sie mit hoher Wahrscheinlichkeit nicht arbeitslos und nicht sozialhilfeabhängig sind, sondern eine selbständige berufliche Karriere planen können, die ihnen Wohlstand ermöglicht. Diese ökonomische Argumentation bildet eine Begründung für die bildungspolitische Forderung, dass möglichst alle Jugendlichen zumindest einen allgemeinbildenden und berufsbildenden Abschluss auf Niveau Sekundarstufe II erreichen (z. B. EDK Richtlinie, 2006).

- In psychologischer Tradition wird oft die Passungs-Norm vertreten (z. B. Holland, 1973; Eccles, 2004; Neuenschwander, in diesem Band): Jugendliche sollen einen Kontext bzw. Bildungsabschluss anstreben, der möglichst stark zu ihren Interessen und Fähigkeiten passt. Es wird postuliert, dass sich Menschen optimal entwickeln, maximale Leistungen erbringen und zufrieden sind, wenn sie eine möglichst hohe Passung zwischen den individuellen Bedürfnissen und Fähigkeiten und den Anforderungen und Angeboten des Kontextes schaffen. Mit der Wahl eines passenden Kontextes finden sie optimale Lern- und Entwicklungsbedingungen vor. Umgekehrt wird gefordert, dass sich die Bildungsangebote am Entwicklungsstand der Jugendlichen ausrichten (Eccles, Midgley, Wigfield, Buchanan, Reuman, Flanagan, et al., 1993).

Diese drei Beispiele zeigen, wie vielfältige Normen postuliert werden, um den Erfolg von Schulübertritten und Bildungsabschlüssen zu bewerten und wie unterschiedlich sie begründet werden. Die Statusnorm und die Schwellennorm minimaler Bildungsabschlüsse resultiert aus einem bildungsbürgerlichen Ideal. Die Passungsnorm orientiert sich hingegen an den Bedürfnissen der Heranwachsenden und den Anforderungen des Kontextes. Sie korrespondiert mit dem eingeführten Person-in-Kontext-Ansatz und beschreibt das Verhältnis des Individuums in seinem Kontext. Daher wird diese Norm in der weiteren Argumentation ins Zentrum gestellt. 


\subsection{Selektion}

Die postmoderne westliche Gesellschaft ist durch eine Vielfalt von Bildungskontexten charakterisiert. In der deutschen und französischen Schweiz ist im Unterschied zum Tessin und zu anderen europäischen Ländern die Sekundarstufe I in Schulniveaus mit unterschiedlich hohen Anforderungen strukturiert. Die Sekundarstufe II unterscheidet allgemeinbildende und berufsbildende Ausbildungsgänge, die je stark ausdifferenziert sind. Mit zunehmendem Alter können Jugendliche zwischen einer wachsenden Zahl von Optionen wählen, die sich in ihren Zielen und Anforderungen unterscheiden.

Die Zuweisung in einen Bildungskontext ist eine Funktion der Schule (Fend, 1981) und basiert auf der Selektion (Auswahl, Gruppierung) von Jugendlichen nach formalen und nicht formalen Kriterien. Diese Kriterien variieren zwischen den einzelnen Stufen (Übertritt in die Sekundarstufe I, Übertritt in Sekundarstufe II, allgemeinbildend bzw. Sekundarstufe II, berufsbildend, usw.) und zwischen den Kantonen (Neuenschwander, 2014).

\subsection{Schultransition}

Die Transition (=Übertritt) bezeichnet den Wechsel von einem (sozialen) Kontext in einen anderen (sozialen) Kontext. Die synchrone Transition meint das Pendeln zwischen zwei Kontexten (z.B. zwischen Schule und Familie). Die diachrone Transition beschreibt das Verlassen eines Kontextes und das Eintreten in einen neuen Kontext (z. B. der Übertritt von der Primarschule in die Sekundarstufe I). Im Unterschied zur Selektionsforschung, in welcher Kriterien der Gruppierung, Chancengleichheit und Statuserwerb durch Bildung diskutiert werden, thematisiert die Transitionsforschung die Belastungen des Übertritts, die Lern - und Entwicklungsprozesse sowie die sozialen Anpassungsprozesse in der Anschlusslösung. Die Transitionsforschung ist daher deutlich von der Forschung zur schulischen Selektion abzugrenzen. Im Anschluss an Bronfenbrenners (1981) Konzept des ökologischen Übertritts umfasst die Transition Veränderungen in vielen Bereichen, etwa der sozialen Rolle, der Beziehungen, der Normierung, der Leistungsanforderungen, der Alltagsgestaltung. Der Kontextwechsel führt insofern zu einer Verdichtung von Entwicklungsprozessen, als er aufgrund der neuen Situation zu einer intensiven Auseinandersetzung mit den eigenen Überzeugungen und dem eigenen Verhalten führt. Entsprechend ist für Bronfenbrenner (1981) ein Übertritt ein Entwicklungsanlass, auch wenn die damit verbundenen Veränderungen mit Belastungen und Unsicherheiten für das Individuum verbunden sind. Damit übereinstimmend 
zeigte zum Beispiel Sirsch (2000), dass die Jugendlichen den Übertritt in die Sekundarstufe I als Herausforderung, aber nicht als Überforderung wahrnehmen. In der Regel genügen die verfügbaren Bewältigungsstrategien und Ressourcen der Jugendlichen zur Bewältigung dieser Herausforderungen.

\subsection{Verhältnis von Individuum und Kontext: Passung}

Das Verhältnis von Individuum und Kontext wurde immer wieder diskutiert (z. B. Heinz, 2000; Lerner, et al., 2005; Eccles \& Roeser, 2011b). Bildungssysteme schaffen Bildungsangebote, zwischen denen Jugendliche im Rahmen ihrer individuellen Möglichkeiten wählen können bzw. zu denen sie aufgrund institutioneller Regeln zugewiesen werden. Mit der Entscheidung bzw. Zuweisung in einen Bildungskontext erhalten sie eine Position und werden mit Normen und Strukturen konfrontiert, an die sie sich anpassen müssen.

Entsprechend resultieren Bildungsverläufe aus einer Dynamik von Gestalten und Anpassen. Diese Dynamik beschreiben Piaget (1947) und Sternberg (1997) als Intelligenzfunktionen, die eine Voraussetzung für das Überleben eines Lebewesens bilden. Nur wenn sich ein Lebewesen an Lebensbedingungen eines Kontextes anpassen kann, kann es seine grundlegenden Bedürfnisse befriedigen und sich entwickeln.

Angewendet auf die Schule heisst das, dass sich Schülerinnen und Schüler optimal entwickeln, wenn die Bildungsangebote auf ihre entwicklungsspezifischen Bedürfnisse, ihre Interessen und ihr Vorwissen abgestimmt sind (Eccles et al., 1993). Diese Passung ist nicht Konformität. Sie ist dynamisch zu verstehen, denn sowohl der Kontext als auch die Kinder verändern sich kontinuierlich. Daher muss die Passung immer neu hergestellt werden. Eine hohe Passung entsteht, wenn die Schule ein Angebot bereitstellt, das möglichst gut mit dem Entwicklungsstand der Schülerinnen und Schüler korrespondiert. Eine hohe Passung ist aber auch eine Leistung der Schülerinnen und Schüler, die soweit sie Wahlmöglichkeiten haben eine Schule bzw. ein Schulniveau nach den individuellen Bedürfnissen wählen, das schulische Umfeld nach ihren Zielen mitzugestalten versuchen und sich an die geltenden Strukturen und Normen der Schule anpassen, d.h. diese übernehmen und in Übereinstimmung damit denken und sich verhalten. 


\section{Arbeitsmodell}

Auf der Grundlage der eingeführten Leitideen wird ein Arbeitsmodell zur Erklärung von Übertritten vorgeschlagen (Abbildung 1). Dieses Arbeitsmodell basiert auf der Annahme, dass mehrere Akteurtypen die Transitionsprozesse steuern: die Jugendlichen, der Kontext Familie, der Kontext Schule und der Kontext Berufsbildung/Beruf. Die Jugendlichen sind die Protagonisten, sie vollziehen aktiv den Übertritt. Der Schulübertritt vollzieht sich in den Strukturen des Bildungssystems. Der Übertritt in die Berufsbildung bzw. den Beruf ist an der Grenze zwischen dem Bildungssystem und dem Berufsbildungssystem angesiedelt. Die Familie ist ein wichtiger, schulergänzender Kontext, der nicht Teil des Bildungs- bzw. Berufsbildungssystems ist, aber Bedingungen und Ergebnisse schulischer Übertritte und Bildungsverläufe wesentlich beeinflusst. Die Steuerung von Schulübertritten durch die Jugendlichen gliedert sich in vier Schritte:

1. Schülerinnen und Schüler verfügen über fachspezifisches Wissen, das sich in entsprechenden schulischen Leistungen, Noten und Verhaltensweisen niederschlägt. Der Wissenserwerb wird durch askriptive Merkmale wie Geschlecht, Schichtzugehörigkeit und allfälligen Migrationshintergrund beeinflusst.

2. Die askriptiven Merkmale und das Wissen führen zu leistungsrelevanten Überzeugungen und Aktivitäten, die den Übertritt vorbereiten.

3. Im dritten Schritt wird die Übertrittsentscheidung gefällt. Danach vollziehen die Jugendlichen den Wechsel in den neuen Kontext.

4. Im vierten Schritt passen sich die Jugendlichen nach dem Übertritt an den neuen Kontext an.

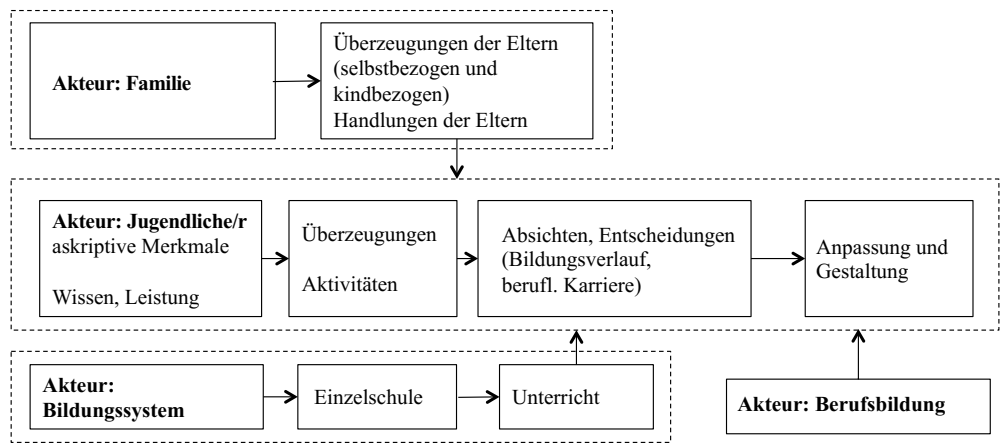

Abb. 1 Arbeitsmodell zur Steuerung von Übertritten 
Im Arbeitsmodell bildet die Familie einen wichtigen ausserschulischen Kontext der Jugendlichen, der die Transitionsprozesse steuert.

Schliesslich postuliert das Modell, dass Bildungsverläufe in die Berufsbildung oder in das Studium und danach in den Beruf münden. Die berufliche Tätigkeit ist also das Ergebnis von Bildungsverläufen. Nach dem Übertritt in die Berufsbildung müssen sich die Jugendlichen an die spezifischen neuen Anforderungen anpassen.

\section{Die Akteure und Akteurinnen}

Im Folgenden werden die verschiedenen Akteure aus Abbildung 1 näher beschrieben.

\subsection{Protagonist: Schüler/in}

Den zentralen Akteur/die zentrale Akteurin (Protagonist) in Schulübertritten bilden die einzelnen Jugendlichen. Sie bereiten sich auf den Übertritt vor, vollziehen diesen und passen sich an die Bedingungen des neuen Kontextes an. Der Übertritt ist institutionell vorstrukturiert, er wird aber durch die praktische Gestaltung durch die Jugendlichen vollzogen. Jugendliche werden dabei von ihren Eltern begleitet. Im Folgenden wird das Arbeitsmodell erläutert.

\subsubsection{Askriptive Merkmale und Wissen}

Die Forschung zur geschlechts- (Eccles, 2007), schichts- und migrationsspezifischen Entwicklung (z. B. Hölscher, 2008, Diehl, Hunkler, Kristen, 2016) ist sehr umfangreich. Geschlecht, Schicht und Migrationshintergrund bilden askriptive Merkmale und Labels, die spezifische Sozialisationserfahrungen indizieren, die früh in der Kindheit beginnen und lebenslang andauern. Sie beeinflussen den Erwerb von fachlichem Wissen, aber auch von Einstellungen der Jugendlichen. Diese Labels beeinflussen aber auch die Einstellungen und Handlungen von Interaktionspartnerinnen und -partnern gegenüber diesen Jugendlichen (Stereotypen), was wesentlich Übertrittsentscheidungen (Maaz, Baumert, Gresch \& McElvany, 2010; Neuenschwander, Gerber, Frank \& Rottermann, 2012) und Anpassungsprozesse nach dem Übertritt steuert. 


\subsection{2 Überzeugungen und Aktivitäten}

Die Überzeugungen von Jugendlichen umfassen erstens Einschätzungen der Schwierigkeit (Erwartungen) und Wertigkeit (Wichtigkeit, Interessantheit, Nutzen, Aufwand) von fachspezifischen und überfachlichen Aufgaben. Sie beeinflussen im Sinne der motivationspsychologisch geprägten Erwartungs-Wert-Theorie von Atkinson \& Raynor (1974), weiterentwickelt von Eccles, Wigfield, und Schiefele (1998), fachspezifische Leistungen, aber auch Bildungsentscheidungen und Bildungsverläufe. Zweitens gehören zu den Überzeugungen auch fachspezifische und soziale Fähigkeitsselbstkonzepte. Drittens bilden allgemeine und fachspezifische Selbstwirksamkeitserwartungen wichtige Überzeugungen in Transitionsprozessen. Damit sind die Einschätzungen gemeint, wie sehr Jugendliche denken, durch das eigene Handeln Wirkungen zu erzeugen und die eigenen Veränderungsprozesse zu gestalten.

Die Überzeugungen von Jugendlichen beeinflussen ihre Handlungen, mit denen sie Übertrittsprozesse gestalten (z. B. Lent et al., 1994). Eine Voraussetzung dafür sind explorative Verhaltensweisen, die Jugendlichen Informationen über die Optionen nach dem Übertritt liefern (Kracke, Olyai, Wesiger, 2008). Dazu gehören beispielsweise das Führen von Gesprächen mit verschiedenen Bezugspersonen, das Lesen von Texten und Sammeln von Informationen usw. Exploratives Verhalten liefert Informationen, auf deren Grundlage zwischen verschiedenen Optionen entschieden und ein Übertritt geplant werden kann (Kracke et al., 2008).

Neben den explorativen Aktivitäten sind auch deviante Verhaltensweisen in Transitionsprozessen zentral. Bedingungen und Ergebnisse von Transitionsprozessen hängen von der Bereitschaft der Jugendlichen ab, die Strukturen und Normen des Kontextes, in dem sie sich befinden bzw. in den sie eintreten werden, zu akzeptieren. Jugendliche mit vielen Unterrichtsstörungen treten bei gleichen Leistungen eher in ein tieferes Schulniveau der Sekundarstufe I über (z. B. Neuenschwander \& Malti, 2009). Beim Übertritt in die Berufsbildung beeinträchtigt deviantes Verhalten die Chancen, eine passende Lehrstelle zu finden, und gefährdet die erfolgreiche Integration in die Ausbildung nach dem Übertritt (Neuenschwander, 2011, Neuenschwander et al., 2012).

\subsubsection{Absichten und Entscheidungen}

Aufgrund von Überzeugungen und Aktivitäten entsteht eine Absicht, ein bestimmter schulischer Abschluss zu erreichen. Diese Absicht ist auf die institutionellen Angebote abgestimmt. Beim Übertritt in die Sekundarstufe I bezieht sich die Absicht auf ein Schulniveau, in welches ein Kind eintritt. Beim Übertritt in die Sekundarstufe II bezieht sich die Absicht auf eine allgemeinbildende oder berufsbildende Ausbildung, 
evtl. auf eine Zwischenlösung. Diese Absicht wird aufgrund der Differenziertheit der Ausbildungsgänge genauer präzisiert.

Die gewählte Ausbildung auf Niveau Sekundarstufe II kann im Hinblick auf (1) den Status (vertikal) und (2) das Berufsfeld bzw. den Berufsinhalt (horizontal) beschrieben werden. Die Entscheidung für die gewählte Ausbildung kann im Hinblick auf das Ausmass der antizipierten Passung zwischen Person und Bildungskontext bzw. Beruf (vgl. Neuenschwander, in diesem Band) und Entscheidungssicherheit (Nägele \& Neuenschwander, 2014) bewertet werden. Die Bewertung der Entscheidung hängt auch davon $\mathrm{ab}$, wie sehr sie mit den eigenen Laufbahnperspektiven korrespondiert bzw. wie sehr sie aus der Zuweisung durch die abgebende oder aufnehmende Institution abweichend zu eigenen Wünschen resultiert.

\subsubsection{Anpassungsprozesse}

Die Übertrittsentscheidung bestimmt, in welchen neuen Bildungskontext Jugendliche eintreten. Diese müssen sich nach dem Übertritt an die neuen Normen und Strukturen anpassen. Dazu gehört, Informationen zur neuen Situation rasch zu verarbeiten. Sie müssen die Anpassungsanforderungen und Gestaltungspielräume im neuen Kontext erkennen und zur Verfolgung der eigenen Ziele nutzen.

Gemäss vorliegendem Arbeitsmodell schliesst der Anpassungsprozess die soziale Integration in den neuen Kontext und den Aufbau von guten Beziehungen zu den neuen Personen mit ein. Wenn die Anpassung misslingt, besteht das Risiko, dass die Jugendlichen die Normen nicht einhalten (z. B. Unterrichtsstörungen, Absenzen, Gewalt u.a. ) und/oder gegebenenfalls diesen Kontext wieder verlassen müssen (Wiederholung eines Schul- oder Ausbildungsjahres, Wechsel in eine Ausbildung mit tieferen Anforderungen, Ausbildungsabbruch oder ähnlich).

\subsection{Kontext: Schule}

\subsubsection{Schulauftrag, Bildungssystem, Lehrplan}

Schultransitionen und Bildungsverläufe sind in das Bildungssystem eingebettet und werden durch dieses gesteuert (Eccles \& Roser, 2011b). Gestützt auf seinen gesetzlichen und pädagogischen Auftrag werden Normen, Strukturen und Lehrpläne geschaffen, die Schultransitionen und Bildungsverläufe strukturieren. Ausgehend von Fend $(1981 ; 2006)$ hat das Bildungssystem den Auftrag, den Schülerinnen und Schülern inhaltliche Bildung (Bildungsfunktion) und Normen (Sozialisations- und Integrationsfunktion) zu vermitteln, sie in die Gemeinschaft aufzunehmen und bei schwerwiegenden Problemen (z. B. Gewalt, familiäre Verwahrlosung, Krankheit 
o.ä.) zu intervenieren (Aufsichts- und Kontrollfunktion). Zudem gruppiert das Bildungssystem gemäss der Selektions- und Allokationsfunktion Schülerinnen und Schüler in unterschiedlich anspruchsvolle Schulformen.

Das Bildungssystem gliedert sich auf der horizontalen Ebene in verschiedene Schulniveaus (Sekundarstufe I) und Ausbildungsgänge (Sekundarstufe II). Die Schulniveaus auf der Sekundarstufe I und die allgemeinbildenden Ausbildungsgänge auf der Sekundarstufe II haben eigene Profile. Sie unterscheiden sich insbesondere in den Leistungsanforderungen, Eintrittsbedingungen und Anschlussoptionen. Bildungssysteme unterscheiden sich in der Durchlässigkeit zwischen Ausbildungsgängen. Beispielsweise zeigte Neuenschwander (2015) grosse Unterschiede in der Durchlässigkeit zwischen verschiedenen Schulniveaus der Sekundarstufe I zwischen den Kantonen. In der Sekundarstufe II ist die Durchlässigkeit zwischen vollzeitschulischen Angeboten (Gymnasium) und berufsbildenden Angeboten formal vorhanden. Eine hohe Durchlässigkeit vergrössert die Vielfalt von Bildungsverläufen, so dass Bildungskontexte stärker auf den individuellen Entwicklungsstand bzw. die Fähigkeiten und Motivation eines Jugendlichen zu einem bestimmten Zeitpunkt abgestimmt werden können. Ein durchlässiges Bildungssystem erlaubt zudem die Korrektur vorangegangener Selektionsentscheidungen.

\subsubsection{Einzelschule}

Das Bildungssystem differenziert sich auf der vertikalen Ebene in Einzelschulen (Fend, 1998). In der Einzelschule wird lokal der kantonale Schulauftrag umgesetzt. Die Art dieser Umsetzung wird durch die Schulführung, das Lehrkollegium mit seinen spezifischen Merkmalen und durch die Zusammensetzung der Schülerschaft bestimmt. Die Einzelschule spielt bei Übertritten eine wichtige Rolle. Die Übertrittsquote in ein Schulniveau der Sekundarstufe I variiert zwischen Primarschulen beträchtlich (Allraum, 2012). Auch die Gymnasialquote variiert je nach Schule der Sekundarstufe I deutlich (z. B. Allraum, 2014). Dies kann teilweise auf die schulspezifische Schülerzusammensetzung zurückgeführt werden. Schulen unterscheiden sich aber auch im Ausmass, wie sie ihre Schülerinnen und Schüler auf einen Übertritt vorbereiten und nach den Standards, die sie bei der Leistungsbewertung anlegen.

Aufnehmende Schulen unterscheiden sich in der Strategie, wie sie neue Schülerinnen und Schüler begrüssen. Sie unterscheiden sich aber auch in inhaltlichen und kindbezogenen Absprachen zwischen den abgebenden und aufnehmenden Schulen (vgl. z. B. Neuenschwander, Fräulin, Belaid, Muischneek-Feissli, 2013, zum Übertritt in die Sekundarstufe I). 


\subsubsection{Unterricht}

Der Schulauftrag wird im Unterrichtssystem realisiert. Das Unterrichtssystem ist ein soziales System, in welchem Schülerinnen und Schüler aufgrund der Interaktion mit den Lehrpersonen und den Gleichaltrigen fachliches Wissen, Kompetenzen und Fertigkeiten erarbeiten und soziale Normen übernehmen (Neuenschwander, 2005). Entsprechend sind die Lernzuwächse je nach Klasse sehr unterschiedlich, so dass die durchschnittlichen Klassentestleistungen in Deutsch und Mathematik im 5. Schuljahr zwischen den Klassen bis zu drei Standardabweichungen variieren, was dem Lernzuwachs von ca. 3 Schuljahren entspricht (Neuenschwander, 2016a). Der Wissenszuwachs in leistungsstarken Klassen ist höher als in leistungsschwachen Klassen (Baumert et al., 2006). Diese unterschiedlichen Klassentestleistungen widerspiegeln sich aber nicht in unterschiedlichen Zeugnisnoten, weil Lehrpersonen je nach Klasse sehr unterschiedliche Beurteilungsstandards verwenden. Je nach Klasse werden die Schülerinnen und Schüler also unterschiedlich stark gefördert, aber nach sehr unterschiedlichen Standards beurteilt, was sich für Schülerinnen und Schüler in leistungsstarken Klassen in tieferen Übertrittschancen in weiterführende Schulen mit höheren Anforderungen auswirkt.

Nach Schulübertritten wechseln die Schülerinnen und Schüler in neue Unterrichtskontexte, an deren Strukturen und Normen sie sich anpassen. Die damit verbundenen Vergleichs- und Lernprozesse schlagen sich beispielsweise in der Position in der Klasse (Rösselet \& Neuenschwander, in diesem Band), im Fähigkeitsselbstkonzept (Marsh, 2005) und in den Noten und Leistungen (Neuenschwander, 2016b) nieder.

\subsection{Familie als ausserschulische Bezugsgruppe}

Die Eltern und Geschwister sind nicht direkt in Schulübertritte involviert, doch begleiten sie als nahe und konstante Bezugspersonen die Schülerinnen und Schüler bei Schulübertritten. Viele Studien belegen den starken Einfluss der Eltern auf die Schülermotivation (Neuenschwander, im Druck), die Schülerleistungen (z.B. Henderson \& Berla, 2004; Neuenschwander \& Goltz, 2008) und das Schülerverhalten im Unterricht (Neuenschwander, Balmer, Gasser, Goltz, Hirt, Ryser \& Wartenweiler, 2005). Gut dokumentiert ist auch der starke Einfluss der Familie auf die Übertrittsentscheidung in die Sekundarstufe I (Neuenschwander et al., 2015) und auf die Berufswahl beim Übertritt in die Sekundarstufe II (z. B. Herzog, Neuenschwander \& Wannack, 2005).

Diese Einflüsse der Familie auf die Schülerinnen und Schüler hängen mit der Schicht der Familie, aber auch mit der Familienstruktur (Zusammensetzung der 
Familie), dem Familienklima und den Beziehungen innerhalb der Familie zusammen. Im Arbeitsmodell wird angenommen, dass der Einfluss dieser Familienmerkmale durch Überzeugungen der Eltern gegenüber sich selber (z. B. berufliche Aspirationen) und gegenüber dem Kind vermittelt wird. Auf der Basis der strukturellen und normativen Merkmale des Familienkontextes und der Überzeugungen der Eltern zeigen die Eltern ein spezifisches Verhalten gegenüber dem Kind: Gut untersucht sind Folgen des Erziehungsstils auf die Schülermotivation (z. B. Wild \& Remy, 2001; Gonzalez \& Wolters, 2006) und die Schülerleistungen (Sy, Gottfried \& Gottfried, 2013). Die Eltern schaffen einen Alltag, in welchem ihre Kinder Einstellungen aufbauen und Gewohnheiten entwickeln, die sich auf die Lern- und Entwicklungsprozesse von Schülerinnen und Schülern in der Schule auswirken (Lord, Eccles, McCarthy, 1994). Im Zentrum stehen nicht intentional arrangierte didaktische Settings im Familienalltag, sondern die Einstellungen, welche Eltern ihren Kindern im Alltag vermitteln und von diesen übernommen werden.

\subsection{Abnehmerinnen: Berufsbildung, Betriebe}

Bildungsverläufe werden mit dem Eintritt in die berufliche Grundbildung unterbrochen oder abgeschlossen. Am Ende der Sekundarstufe I erfüllen die meisten Schülerinnen und Schüler die Voraussetzungen, ein Bewerbungsverfahren in die Berufsbildung erfolgreich zu durchlaufen. Die berufliche Grundbildung definiert Ausbildungsgänge mit unterschiedlichen inhaltlichen Profilen, Anforderungen und spezifischen Zugangsvoraussetzungen, die in einem Selektionsverfahren überprüft werden (z.B. Bewerbungsunterlagen und Bewerbungsgespräche). Diese Selektionsverfahren unterscheiden sich von den innerschulischen Selektionsverfahren in mehrerer Hinsicht (Neuenschwander, 2014). Sie basieren nicht primär auf der Beurteilung schulischer Leistungen, sondern auf Anforderungen im jeweiligen Berufsfeld. In vielen Berufen spielen überfachliche Kompetenzen eine zentrale Rolle (Neuenschwander \& Wismer, 2010; Neuenschwander \& Hermann, 2013). Die Selektionsverfahren sind nicht staatlich, sondern privat organisiert. Die Betriebe können im Rahmen der geltenden Gesetzgebung Lehr- und Arbeitsstellen nach ihren eigenen Regeln vergeben und müssen ihre Entscheidungen nicht transparent treffen oder gar rechtfertigen. Während bei schulischen Übertritten in der Regel nur wenige Ausbildungsoptionen zur Verfügung stehen, aber alle Jugendlichen einer dieser Optionen zugeordnet werden können, stehen im Lehrstellen- und Arbeitsmarkt viele Optionen zur Auswahl, wobei kein Anrecht auf Zugang zu einer oder mehrerer dieser Optionen besteht. In der Regel begünstigen vorgängige einschlägige 
betriebliche Erfahrungen (z. B. Schnupperlehren) und berufsspezifisches Wissen die Chancen auf eine Lehr- oder Arbeitsstelle (vgl. Nägele \& Stalder, in diesem Band).

Mit dem Eintritt in die berufliche Grundbildung sind Jugendliche mit vielfältigen Änderungen konfrontiert: Arbeitszeiten, Leistungsanforderungen, Rolle in der Arbeitsgruppe, Arbeitsinhalte usw. Allerdings gelingt den meisten Jugendlichen mit Direktübertritt die Anpassung an die neue Ausbildung gut (Nägele \& Neuenschwander, 2015). Die Anpassungsprozesse nach Eintritt in den Lehrbetrieb werden wesentlich durch Feedback und Integrationsanforderungen in den Ausbildungsbetrieb gesteuert (Nägele \& Neuenschwander, 2014). In der Berufsfachschule steht die Auseinandersetzung mit hohen Leistungsanforderungen und neuen Vergleichsgruppen im Zentrum.

\section{$4 \quad$ Schlussfolgerungen}

Es wird ein Arbeitsmodell zur Analyse von Schultransitionen vorgestellt, aus dem zahlreiche Forschungsfragen und Unterstützungsmassnahmen abgeleitet werden können. Das Modell bildet auf einem mittleren Abstraktionsniveau ein Analyseraster, das zentrale Begriffe und Prozessaussagen enthält, mit denen Bedingungen und Ergebnisse von Übertritten in die Sekundarstufe I und II beschrieben und erklärt werden können. Eine Besonderheit besteht darin, dass das Modell annimmt, dass Menschen in einem sozialen Kontext situiert sind, weshalb es nicht nur kognitive, sondern auch soziale Dimensionen enthält.

Der Nutzen des Modells liegt in der Fokussierung auf ausgewählte, aber wichtige Erklärungskonzepte, die das Verständnis von Übertritten vertiefen, neue Forschung ermöglichen und eine zielgerichtete Begleitung von Jugendlichen bei Übertritten ermöglichen. D. h. , das Erklärungsmodell liefert eine Basis für Interventionsprogramme, wie beispielsweise benachteiligte oder im Berufswahlprozess verzögerte Jugendliche unterstützt werden können. Es liefert zudem Hinweise, wie Lehrpersonen und Eltern wirksam Jugendliche bei Übertritten und beim Eintritt in die Berufsbildung begleiten können.

Das Modell kann nicht mit einer einzelnen empirischen Studie bestätigt werden. Teile des Modells bewährten sich in früheren empirischen Studien (vgl. die zitierte Literatur). Andere Teile müssen in zukünftigen Studien überprüft und gegebenenfalls modifiziert werden. Dadurch kann das Modell weiter entwickelt und präzisiert werden. Die gewählte Abstraktionshöhe erlaubt aber einerseits eine gewisse Allgemeingültigkeit, andererseits die Formulierung konkreter Hypothesen, die überprüft werden können. 


\section{Literatur}

Allraum, J. (2012). Bildungsstatistik Kanton Bern: Sekundarschulanteil im 7. Schuljahr nach Wohnort 2011. Bern: Erziehungsdirektion des Kantons Bern.

Allraum, J. (2014). Bildungsstatistik Kanton Bern: Übertrittsquoten ins Gymnasium nach Wohnort 2013. Bern: Erziehungsdirektion Bern.

Atkinson, J. W. \& Raynor, J. D. (1974). Motivation and achievement. Washington: Winston.

Baumert, J., Stanat, P. \& Watermann, R. (2006). Schulstruktur und die Entstehung differenzieller Lern- und Entwicklungsmilieus. In J. Baumert, P. Stanat \& R. Waterman (Hrsg.), Herkunftsbedingte Disparitäten im Bildungswesen: Differenzielle Bildungsprozesse und Probleme der Verteilungsgerechtigkeit. (S.95-188). Wiesbaden: VS-Verlag.

Bronfenbrenner, U. (1981). Die Ökologie der menschlichen Entwicklung. Natürliche und geplante Experimente. Stuttgart: Klett-Cotta.

Diehl, C., Hunkler, C. \& Kristen, C. (Hrsg.). (2016). Ethnische Ungleichheiten im Bildungsverlauf. Wiesbaden: Springer.

Eccles, J. S. (2004). Schools, academic motivation, and stage-environment fit. In R. M. Lerner \& L. Steinberg (Eds.), Handbook of adolescent psychology (2nd ed., Vol. 2, pp. 125-153). Hoboken, NJ: Wiley.

Eccles, J. S. (2007). Where are all the women? Gender differences in participation in physical science and engineering. In S. J. Ceci \& W. M. Williams (Eds.), Why are not more women in science? (pp. 199-210). Washington: American Psychological Association.

Eccles, J. S., \& Roeser, R. W. (2011a). Schools as developmental contexts during adolescence. Journal of Research on Adolescence, 21(1), 225-241.

Eccles, J. S., \& Roeser, R. W. (2011b). School and community influences on human development. In M. H. Bornstein \& M. E. Lamb (Eds.), Developmental science: An advanced textbook (6 ed., pp. 571-644). New York and Hove: Psychology Press.

Eccles, J. S., Midgley, C., Wigfield, A., Buchanan, C. M., Reuman, D., Flanagan, C., et al. (1993). Development during adolescence: The impact of stage-environment fit on young adolescents' experiences in schools and in families. American Psychologist, 48, 90-101.

Eccles, J. S., Wigfield, A. \& Schiefele, U. (1998). Motivation to succeed. In W. Damon \& N. Eisenberg (Eds.), Handbook of child psychology: Social, emotional, and personality development (Vol. 3, pp. 1017-1095). New York: Wiley.

Fend, H. (1981). Theorie der Schule (2. Aufl.). München: Urban \& Schwarzenberg.

Fend, H. (1998). Qualität im Bildungswesen. Schulforschung zu Systembedingungen, Schulprofilen und Lehrerleistung. Weinheim: Juventa.

Fend, H. (2006). Neue Theorie der Schule. Einführung in das Verstehen von Schulsystemen. Wiesbaden: VS-Verlag.

Gonzalez, A.-L. \& Wolters, C. A. (2006). The relation between perceived parenting practices and achievement motivation in mathematics. Journal of Research in Childhood Education, 21(2), 203-217. doi:10.1080/02568540609594589

Henderson, A. T. \& Berla, N. (Eds.). (2004). A new generation of evidence. The family is critical to student achievement. US: National committee for citizens in education.

Heinz, W. R. (2000). Selbstsozialisation im Lebenslauf. Umrisse einer Theorie biografischen Handelns. In E. M. Hoerning (Hrsg.), Biografische Sozialisation (S. 165-186). Stuttgart: Lucius \& Lucius Verlagsgemeinschaft.

Herzog, W., Neuenschwander, M. P. \& Wannack, E. (2006). Berufswahlprozess. Bern: Haupt. 
Holland, J. L. (1973). Making vocational choices, a theory of careers. Englewood Cliffs: Prentica-Hall.

Hölscher, B. (2008). Sozialisation, Sozialisationskontexte, schichtspezifische Sozialisation. In H. Willems (Hrsg.), Lehr(er)buch Soziologie (Vol. 2, pp. 747-772). Wiesbaden: VS-Verlag für Sozialwissenschaften.

Kracke, B., Olyai, N. \& Wesiger, J. (2008). Stand der Berufswahl und Qualität des berufsbezogenen Explorationsverhaltens im Jugendalter. Psychologie in Erziehung und Unterricht, 55(1), 51-60.

Lave, J. \& Wenger, E. (1991). Situated learning. Legitimate peripheral participation. Cambridge: Cambridge University Press.

Lent, R. W., Brown, S. D. \& Hackett, G. (1994). Toward a unifying social cognitive theory of career and academic interest, choice, and performance. Journal of Vocational Behavior, 45(1), 79-122.

Lerner, R. M. \& Walls, T. (1999). Revisiting individuals as producers of their development: From dynamic interactionism to developmental systems. In J. Brandtstädter \& R. M. Lerner (Eds.), Action and self-development: Theory and Research through the life span (pp. 3-36). Thousand Oaks: Sage.

Lerner, R. M., Theokas, C. \& Jelicic, H. (2005). Youth as active agents in their own positive development: A developmental systems perspective. In K. Rothermund, D. Wentura \& W. Greve (Eds.), The adaptive self: Personal continuity and intentional self-development (pp. 31-47). Ashland, OH: Hogrefe \& Huber Publisher.

Lord, S., Eccles, J. S. \& McCarthy, K. (1994). Surviving the junior high school transition: Family processes and self-perceptions as protective and risk factors. Journal of Early Adolescence, 14, 162-199.

Maaz, K., Baumert, J., Gresch, C. \& McElvany, N. (Hrsg.). (2010). Der Übertritt von der Grundschule in die weiterführende Schule: Leistungsgerechtigkeit und regionale, soziale und ethnisch-kulturelle Disparitäten. Zusammenfassung der zentralen Befunde. Bonn: Bundesministerium für Bildung und Forschung, Referat Bildungsforschung.

Marsh, H. W. (2005). Big-fish-little-pond effect on academic self-concept. Zeitschrift für Pädagogische Psychologie, 19(3), 119-127.

Nägele, C. \& Neuenschwander, M. P. (2014). Adjustment processes and fit perceptions as predictors of organizational commitment and occupational commitment of young workers. Journal of Vocational Behavior, 85, 385-393. doi:dx.doi.org/10.1016/j.jvb.2014.08.011

Nägele, C. \& Neuenschwander, M. P. (2015). Passt der Beruf zu mir? In K. Häfeli, M. P. Neuenschwander, \& S. Schumann (Hrsg.), Berufliche Passagen im Lebenslauf(S. 49-74). Wiesbaden: VS Verlag.

Nägele, C. \& Stalder, B. E. (2017). Übergänge in die Berufs- und Allgemeinbildung: ein Arbeitsmodell. In M. P. Neuenschwander \& C. Nägele (Hrsg.), Bildungsverläufe von der Einschulung in die Erwerbstätigkeit: Theoretische Ansätze - Befunde - Beispiele (S. 21-36). Wiesbaden: Springer VS.

Neuenschwander, M. P. \& Gerber, M. (2014). Schulische Vorbereitung auf die berufliche Sozialisation im Lehrbetrieb. Unterrichtswissenschaft, 42(3), 244-260.

Neuenschwander, M. P. \& Goltz, S. (2008). Familiäre Bedingungen von Schülerleistungen: Ein typologischer Ansatz. Psychologie in Erziehung und Unterricht, 55(4), 265-275.

Neuenschwander, M. P. \& Hermann, M. (2013). Der richtige Mix aus Kompetenzen und Persönlichkeit. Panorama, 27(4), 23. 
Neuenschwander, M. P. \& Malti, T. (2009). Selektionsprozesse beim Übergang in die Sekundarstufe I und II. Zeitschrift für Erziehungswissenschaft, 12(2), 216-232.

Neuenschwander, M. P. \& Wismer, N. (2010). Selektionskriterien: Wichtige Rolle der überfachlichen Kompetenzen. Panorama(1), 16-17.

Neuenschwander, M. P. (2005). Unterrichtssystem und Unterrichtsqualität. Bern: Haupt.

Neuenschwander, M. P. (2011). Determinanten der Passungswahrnehmung nach dem Übergang in die Sekundarstufe II. Schweizerische Zeitschrift für Bildungswissenschaften, 33(3), 401-419.

Neuenschwander, M. P. (2014). Selektionsentscheidungen beim Übergang in die Sekundarstufe I und in den Arbeitsmarkt im Vergleich. In M. P. Neuenschwander (Hrsg.), Selektion in Schule und Arbeitsmarkt (S. 63-98). Zürich: Rüegger.

Neuenschwander, M. P. (2015). Schulformwechsel - Thesen zur Durchlässigkeit in der Sekundarstufe I. Schulverwaltung, 20(4), 110-112.

Neuenschwander, M. P. (2016a). Beurteilung und Beurteilungsverzerrungen. Profil(3), 5-9.

Neuenschwander, M. P. (2016b). Bildungsungleichheit am Beispiel der Leistungsentwicklung in Deutsch und Mathematik beim Übergang in die Sekundarstufe I. In B. Ziegler (Hrsg.), (Un-)Gleichheiten in der Demokratie (S.95-118). Zürich: Schulthess-Verlag.

Neuenschwander, M. P. (2017). Anpassungsprozesse beim Übergang in die Sekundarstufe I. In M. P. Neuenschwander \& C. Nägele (Eds.), Bildungsverläufe von der Einschulung in die Erwerbstätigkeit (pp. 143-161). Wiesbaden: VS Verlag für Sozialwissenschaften.

Neuenschwander, M. P. (2017). Lern- und Leistungszielorientierung beim Übergang in die Sekundarstufe I: Längsschnittliche Befunde zur Bedeutung von Belastungen und Erziehungsverhalten von Eltern. Schweizerische Zeitschrift für Bildungswissenschaften, 39(2).

Neuenschwander, M. P., Balmer, T., Gasser, A., Goltz, S., Hirt, U., Ryser, H., \& Wartenweiler, H. (2005). Schule und Familie - was sie zum Schulerfolg beitragen. Bern: Haupt.

Neuenschwander, M. P., Fräulin, J., Belaid, S. \& Muischneek-Feissli, B. (2013). Wirkungen der Selektion - qualitative Begleitstudie. 3. Zwischenbericht. Solothurn: PH FHNW.

Neuenschwander, M. P., Gerber, M., Frank, N. \& Rottermann, B. (2012). Schule und Beruf. Wiesbaden: VS-Verlag.

Piaget, J. (1947). La psychologie de l'intelligence. Paris: Colin.

Reinmann-Rothmeier, G. \& Mandl, H. (2002). Analyse und Förderung kooperativen Lernens in netzbasierten Umgebungen. Zeitschrift für Entwicklungspsychologie und Pädagogische Psychologie, 34, 44-57.

Rösselet, S., \& Neuenschwander, M. P. (2017). Akzeptanz und Ablehnung von Schülerinnen und Schülern beim Übertritt in die Sekundarstufe I. In M. P. Neuenschwander \& C. Nägele (Eds.), Bildungsverläufe von der Einschulung in die Erwerbstätigkeit: Theoretische Ansät$z e$ - Befunde - Beispiele (pp. 103-121). Wiesbaden: VS Verlag für Sozialwissenschaften.

Sirsch, U. (2000). Probleme beim Schulwechsel. Münster: Waxmann.

Sternberg, R. J. (1997). The concept of intelligence and its role in lifelong learning and success. American Psychologist, 52(2), 1030-1038.

Sy, S. R., Gottfried, A. W. \& Gottfried, A. E. (2013). A transactional model of parental involvement and children's achievement from early childhood through adolescence. Parenting, 13(2), 133-152. doi:10.1080/15295192.2012.709155

Welsch, W. (1988). Unsere postmoderne Moderne. Weinheim: VCH Verlagsgesellschaft.

Wild, E. \& Remy, K. (2001). Affektive und motivationale Folgen der Lernhilfen und lernbezogenen Einstellungen der Eltern. Unterrichtswissenschaft, 30, 27-51. 
Open Access Dieses Kapitel wird unter der Creative Commons Namensnennung 4.0 International Lizenz (http://creativecommons.org/licenses/by/4.0/deed.de) veröffentlicht, welche die Nutzung, Vervielfältigung, Bearbeitung, Verbreitung und Wiedergabe in jeglichem Medium und Format erlaubt, sofern Sie den/die ursprünglichen Autor(en) und die Quelle ordnungsgemäß nennen, einen Link zur Creative Commons Lizenz beifügen und angeben, ob Änderungen vorgenommen wurden.

Die in diesem Kapitel enthaltenen Bilder und sonstiges Drittmaterial unterliegen ebenfalls der genannten Creative Commons Lizenz, sofern sich aus der Abbildungslegende nichts anderes ergibt. Sofern das betreffende Material nicht unter der genannten Creative Commons Lizenz steht und die betreffende Handlung nicht nach gesetzlichen Vorschriften erlaubt ist, ist für die oben aufgeführten Weiterverwendungen des Materials die Einwilligung des jeweiligen Rechteinhabers einzuholen.

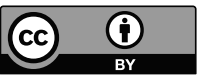




\section{Übergänge in die Berufsbildung - Ein Arbeitsmodell}

Christof Nägele und Barbara E. Stalder

\section{Zusammenfassung}

In diesem Kapitel wird ein Arbeitsmodell zum Übergang von der obligatorischen Schule in die Berufsbildung vorgestellt. Das Modell erlaubt eine Systematisierung und Integration bestehender Phasen- und Strukturmodelle der Wahl einer nachobligatorischen Ausbildung, des Übergangs und des Ausbildungsbeginns. Das Modell strukturiert den Übergang anhand von Themen, Aufgaben und Rollen verschiedener Akteurinnen und Akteure. Auf der Sekundarstufe I beschäftigen sich die Jugendlichen im Rahmen der Schul- und Berufsorientierung mit den drei Themen Orientieren, Bewerben/Anmelden und Vorbereiten, auf der Sekundarstufe II mit den Themen Ankommen, Lernen und Leisten sowie Weiterkommen. Jedes dieser Themen beinhaltet typische Aufgaben, die Jugendliche zu bearbeiten haben. Je nach Thema und Aufgabe haben individuelle und institutionelle Akteurinnen und Akteure unterschiedliche Rollen. Vor Beginn der Allgemein- oder Berufsbildung ist ein wichtiges Ziel, ein Gefühl der Passung mit der zukünftigen Ausbildung zu entwickeln (pre-entry fit). Nach Beginn der Ausbildung sind die Anpassung und das Finden der neuen Passung (post-entry fit) sowie die soziale Integration wichtig.

\section{Schlagworte}

Obligatorische Schule, Allgemeinbildung, Berufsbildung, Arbeitsmodell, Passung, Soziale Integration 


\section{Arbeitsmodell von der obligatorischen Schule in die Berufsbildung}

Die systematische Auseinandersetzung mit der Schul- und Berufswahl erfolgt gemäss aktueller Lehrpläne in der Schweiz auf der Sekundarstufe I (Nägele \& Schneitter, 2016). Aus einer Lebensverlaufsperspektive werden bei der Wahl der nachobligatorischen Ausbildung für die weitere Gestaltung der Laufbahn richtungsweisende Weichen gestellt. Damit sind lebenslange persönliche und berufliche Entwicklungsprozesse angesprochen, die von Individuen vor dem Hintergrund der aktuellen wirtschaftlichen, politischen und sozialen Situation, individueller Fähigkeiten, Werte und Ziele sowie der Eingebundenheit einer Person in ihr soziales Umfeld aktiv gestaltet werden (Stalder \& Nägele, 2015; Stringer, Kerpelman, \& Skorikov, 2011; Vondracek, Lerner, \& Schulenberg, 1986).

Zur Entwicklung des Arbeitsmodells zum Übergang von der obligatorischen Schule in die Berufsbildung wird erstens dargestellt, welche Bildungswege im Übergang von der obligatorischen Schule in die Ausbildung auf der Sekundarstufe II, in die Ausbildung auf Tertiärstufe (Höhere Bildung, Tertiär B und A) und Weiterbildung möglich sind (Nägele \& Bierschenk, 2014), da diese die Alternativen der Jugendlichen im Übergang strukturieren. Zweitens wird das Arbeitsmodell von der obligatorischen Schule in die Berufsbildung eingeführt. Das Arbeitsmodell integriert Ansätze eines Phasenmodells der Berufswahl (Herzog, Neuenschwander, \& Wannack, 2001; Neuenschwander, 2008), eines Modells zu Selektion, Übergang und Eintritt in die Sekundarstufe II (Stalder \& Schmid, 2016) und Überlegungen zur Wahl eines Berufs (Parsons, 1909). Dazu werden im Arbeitsmodell Themen, Aufgaben sowie Akteurinnen und Akteure identifiziert. Themen sind übergeordnete Gegenstände, mit denen sich die Jugendlichen im Übergang beschäftigen müssen, Aufgaben sind einzelne zu einem Thema gehörende Tätigkeiten. Eltern, Lehrpersonen, Berufsbildner/innen und institutionelle Akteurinnen und Akteure (Wettstein, Schmid, \& Gonon, 2014) lenken, unterstützen und begleiten die Schüler/ innen und Lernenden, indem sie direkt intervenieren oder das Bildungssystem gestalten. Zudem werden im Arbeitsmodell dem Laufbahnprozess übergeordnete Prozesse beschrieben. Es sind dies die Prozesse der Anpassung und des Findens einer Passung der Person zum Beruf und zum Betrieb (Edwards \& Shipp, 2007; Nägele \& Neuenschwander, 2015; Stalder \& Schmid, 2016) sowie der Entwicklung der sozialen Zugehörigkeit zu einer Gruppe und einer eigenen Identität (Nägele, 2015; Wenger, 2008). Selbst-regulative Prozesse, in denen die aktuelle Situation mit dem gewünschten Ziel verglichen wird, lösen Aktivitäten im Denken und Handeln aus und sind die treibende Kraft für Veränderungen (Carver \& Scheier, 2002; Locke \& Latham, 2002). Die Suche nach Passung, das Verlieren der Passung und Wieder- 
finden derselben kann so viele berufliche Veränderungen und Übergange erklären (Kristof-Brown, Zimmerman, \& Johnson, 2005). Die soziale Integration ist eine Voraussetzung für das Lernen (Crosnoe, 2011; Wenger, McDermott, \& Snyder, 2002).

\section{$2 \quad$ Bildungswege}

Die Bildungswege durch die obligatorische und nachobligatorische Ausbildung sind durch das Bildungssystem vorgegeben. Das Bildungssystem auf der Sekundarstufe I zeichnet sich in den meisten Schweizer Kantonen durch eine starke Gliederung in unterschiedliche Schulniveaus aus. Im Gegensatz dazu bieten sich auf der Sekundarstufe II, vor allem auch in der Berufsbildung, vielfältige Möglichkeiten, die Bildungswege an individuelle, fachliche, familiäre oder soziale Voraussetzungen anzupassen. Dies wird durch eine grundsätzlich offene und flexible Gestaltung des Bildungssystems ermöglicht (Dubs, 2006) (Abbildung 1, siehe auch www.edudoc. ch). Allgemeinbildende Ausbildungen führen zu einer Maturität. Das gymnasiale Maturitätszeugnis eröffnet grundsätzlich einen Zugang zur Hochschulbildung (Tertiär A). Die berufliche Grundbildung mit Berufsmaturität und die Wirtschaftsmittelschulen führen zu einer Doppelqualifikation, auch hybride Qualifikation

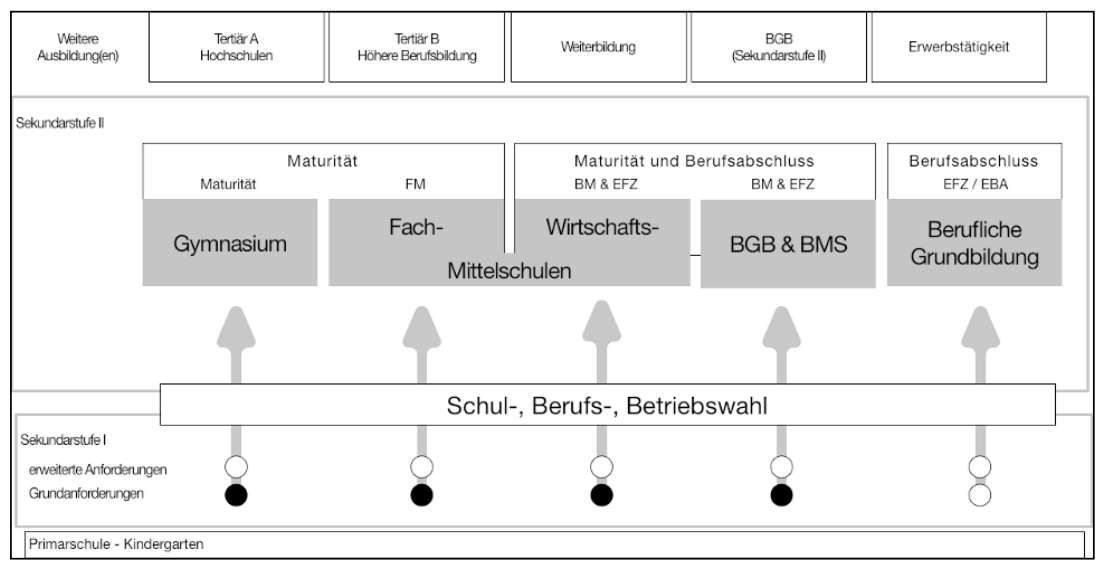

Abb. 1 Bildungswege

Bemerkung. Schwarz = kein Zugang, FM Fachmaturität, BM= Berufsmaturität, BGB = Berufliche Grundbildung, EFZ = Eidgenössisches Fähigkeitszeugnis, EBA = Eidgenössisches Berufsattest 
genannt (Gonon, 2013), und ermöglichen den Zugang zu Fachhochschulen und Pädagogischen Hochschulen (Tertiär A). Für Jugendliche mit einer beruflichen Grundbildung ohne Berufsmaturität stehen im Bereich der höheren Berufsbildung Entwicklungsmöglichkeiten offen (Tertiär B) (Nägele \& Bierschenk, 2014). Aktuelle Studien zeigen, dass sich viele junge Erwachsene nach der beruflichen Grundbildung weiter ausbilden und sich beruflich verändern (Hupka-Brunner, Scharenberg, Meyer, \& Müller, 2015; Müller \& Schweri, 2009). Das heisst, dass die im Bildungssystem angelegte Durchlässigkeit genutzt wird.

\subsection{Arbeitsmodell zum Übergang von der obligatorischen Schule in die Berufsbildung}

Das Arbeitsmodell von der obligatorischen Schule in die Berufsbildung umfasst die Zeitspanne von Beginn der Sekundarstufe I bis ca. sechs Monate nach Beginn der beruflichen Grundbildung sowie die Benennung von Themen, Aufgaben und Akteurinnen und Akteuren. Der Vorteil des Arbeitsmodells liegt darin, dass Prozesse der Anpassung und Integration vor und nach dem Übergang als aufeinander bezogen beschrieben werden. Die im Arbeitsmodell beschriebenen Themen können in einer zeitlichen Abfolge gesehen werden. Jugendliche können aber auch gleichzeitig mehrere Themen bearbeiten, respektive Themen je nach Verlauf der Schul- und Berufsorientierung auf der Sekundarstufe I resp. dem Einstieg in die Ausbildung auf der Sekundarstufe II wieder aufnehmen. Es ist z. B. nicht notwendig, das Thema Orientieren abzuschliessen, bevor man sich bewirbt. Ein Thema ist deshalb auch nicht durch eine Entscheidung abgeschlossen. Aufgrund der Komplexität der Entscheidung für die weitere Ausbildung werden fortlaufend Entscheidungen gefällt und auch wieder revidiert.

Über die Differenzierung einzelner Aufgaben in den einzelnen Themen kann die Rolle unterschiedlicher Akteurinnen und Akteure dargestellt werden. So haben die Eltern gemeinhin einen sehr grossen Einfluss auf die Wahl der Ausbildung auf der Sekundarstufe II (Neuenschwander, Gerber, Frank, \& Rottermann, 2012). Betrachtet man den Einfluss anhand des vorgestellten Arbeitsmodells differenzierter, so kann z. B. dargestellt werden, dass beim Thema Orientieren der Einfluss der Eltern vor allem bei Entscheidungen wichtig ist, die im familiären Nahbereich gefällt werden (Beierle, 2013). Bei der Informationssuche hingegen spielen die Lehrpersonen und die Berufs-, Studien- und Laufbahnberatung eine wichtigere Rolle. Durch diese thematisch gruppierte, differenzierte Aufschlüsselung der Aufgaben und Rollen können auch Schnittstellen unterschiedlicher Akteurinnen und Akteure aufgezeigt werden, z. B. zwischen der Volksschule und der Allgemeinbildung oder den 
Berufsfachschulen. Das Arbeitsmodell wird im Folgenden schwerpunktmässig mit Blick auf die Berufsbildung dargestellt, lässt sich für die Allgemeinbildung jedoch anpassen (Abbildung 2).

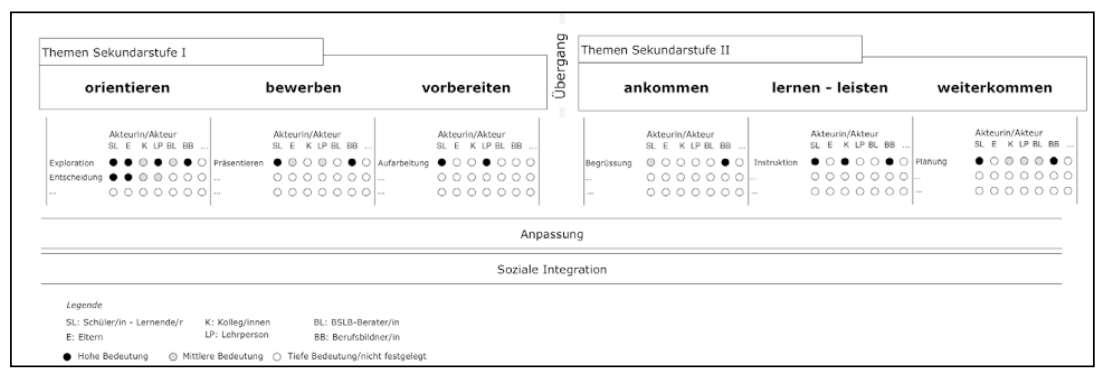

Abb. 2 Generisches Arbeitsmodell, mit Themen, Aufgaben sowie Akteurinnen und Akteuren im Übergang von der obligatorischen Schule in die Berufsbildung

\section{Themen und Aufgaben auf der Sekundarstufe I}

Beim Thema Orientieren ist eine wichtige Aufgabe, dass sich Jugendliche mit der inhaltlichen Ausrichtung ihrer weiteren Ausbildung und der Frage nach der Ausbildungsinstitution auseinandersetzen. Dieses Orientieren wird in der Deutschschweiz oft als „Berufskunde“ oder „Berufswahl“ bezeichnet, im Lehrplan 21 als „berufliche Orientierung“ (D-EDK, 2015). Der Fokus liegt gemäss Lehrplan auf der Wahl eines Berufs, das heisst einer beruflichen Grundbildung (Nägele \& Schneitter, 2016). Typischerweise erkunden Schüler/innen zuerst ihre eigenen Interessen und fachlichen Kompetenzen, danach ihre beruflichen Optionen und entscheiden sich schliesslich für einen passenden Beruf. Dieses Vorgehen birgt jedoch Gefahren.

Erstens wird mit der Fokussierung auf Interesssen die Tendenz gefördert, dass sich Jugendliche vor allem im Rahmen dessen orientieren, was sie bereits kennen. Denn Interessen für unterschiedliche Tätigkeiten und unterschiedliche Berufe entwickeln sich aufgrund einer Auseinandersetzung mit eigenen Erfahrungen und Rückmeldungen in unterschiedlichen Situationen (Lent, Brown, \& Hackett, 2002). Damit Schüler/innen Interessen ausserhalb des ihnen bekannten und durch die Eltern und Lehrpersonen strukturierten Umfelds entwickeln können, müssen sie die Möglichkeit haben, dieses erweiterte Umfeld zu erkunden. Das Orientieren sollte deshalb mit einer Exploration dessen beginnen, was existiert, den Schüler/ 
innen aber nicht bekannt ist. Dies in Anlehnung an Modelle der Beratung, in denen vor einer Entscheidung das Screening einer grossen Bandbreite von machbaren Alternativen an erster Stelle steht (Gati \& Tal, 2008). Es geht also darum, den Prozess der vorschnellen Einengung der Schul- und Berufsorientierung auf wenige Alternativen zu verzögern; eine Einengung, wie sie von Gottfredson (2002) mit dem Bereich der erlaubten Wahl von Berufsfeldern problematisiert worden ist. Zweitens wird mit der Fokussierung auf die Berufswahl die Wahl der Ausbildungsinstitution weitgehend vernachlässigt (Stalder \& Schmid, 2016). Gemäss gängiger Konzepte erfolgt die Wahl des Betriebs bei einer beruflichen Grundbildung erst dann, wenn sich die Jugendlichen für einen Beruf entschieden haben und sich auf Lehrstellen bewerben. Konsequenterweise wird die Auseinandersetzung mit der Entscheidung für einen passenden Betrieb zum Beispiel im Berufswahltagebuch (Jungo \& Egloff, 2015) kaum thematisiert. Vielmehr werden die Jugendlichen darauf hingewiesen, dass zum Beispiel in der Schnupperlehre der Beruf und nicht etwa der Betrieb zu beurteilen sei (S. 81). Aktuelle Studien zeigen jedoch, dass eine erfolgreiche Anpassung an die Anforderungen der beruflichen Grundbildung massgeblich auch von der passenden Betriebswahl abhängt (Nägele \& Neuenschwander, 2015; Stalder \& Schmid, 2016). Das heisst, dass der Wahl des «richtigen» Ausbildungsbetriebs eine ebenso bedeutende Rolle zukommen sollte wie der Wahl des «richtigen» Berufs.

Die Fokussierung auf die Berufswahl führt weiter oft dazu, dass sich Schüler/ innen, die sich für eine Allgemeinbildung interessieren, auf der Sekundarstufe I weniger mit ihrer weiteren Laufbahn auseinandersetzen. Sie müssen sich im Übergang von der Sekundarstufe I in die Sekundarstufe II noch nicht für einen Beruf entscheiden. In einigen Kantonen sind diese schulisch leistungsstarken Jugendlichen von der Schul- und Berufsorientierung auf der Sekundarstufe I dispensiert (Nägele \& Schneitter, 2016). Aus einer Lebenslaufperspektive wäre es angezeigt, dass sich alle Schüler/innen mit ihrer beruflichen Zukunft intensiver auseinandersetzen und so nicht in eine Allgemeinbildung einmünden, ohne sich mit Überzeugung dafür entschieden zu haben. Dies erhöht das Risiko eines späteren Abbruchs der Ausbildung (Stamm, Holzinger-Neulinger, Suter, \& Stroezel, 2007).

Das zweite Thema ist das Bewerben auf eine Lehrstelle oder die Anmeldung bei einer Schule. Während schulische Ausbildungen meist aufgrund der Noten und Empfehlungen der Lehrpersonen und allenfalls Aufnahmeprüfungen zugänglich sind, sind berufliche Grundbildungen in der Regel über eine Schnupperlehre, eine formale, schriftliche Bewerbung und dem erfolgreichen Durchlaufen eines branchen- oder betriebsspezifischen Bewerbungs- und Selektionsverfahrens zugänglich (Imdorf, 2007). Im Bewerbungsverfahren stellen sich den Schüler/innen mehrere Aufgaben. So müssen sich die Jugendlichen schriftlich im Bewerbungsdossier und persönlich im Vorstellungsgepräch präsentieren, damit sie eine reelle Chance auf 
den Zuschlag für eine Lehrstelle haben. Sie müssen sich interessiert und motiviert zeigen (Stalder, 2000), da sie damit ihre Ausbildungsbereitschaft und Beschäftigungsfähigkeit signalisieren (Fugate, Kinicki, \& Ashforth, 2004). Betriebe legen bei der Bewerbung zudem einen sehr hohen Wert auf die Einhaltung sozialer Regeln, Normen und Tugenden (Stalder, 2000; 2002; Stricker \& Stalder, 2009). Betriebe signalisieren ihrerseits, dass sie den Lernenden attraktive Ausbildungsbedingungen anbieten, was insbesondere bei einer knappen Bewerberlage entscheidend ist.

Nach der Bewerbung folgt für die Jugendlichen, die eine Lehrstelle gefunden haben, oft eine mehrere Wochen oder Monate dauernde Zeitspanne der Vorbereitung. Lehrverträge werden von den Betrieben, vor allem bei schulisch stärkeren Schüler/ innen, frühzeitig abgeschlossen oder durch Vorverträge zugesichert. Diese Zeit kann im negativen Fall für einige Jugendliche zu einer Art Leerzeit vor der Lehrzeit werden. Denn sie haben ihr Ziel, eine gute Lehrstelle zu finden, bereits erreicht. Es hängt in dieser Situation oft von den Lehrpersonen ab, wie diese Jugendlichen das letzte Schuljahr gestalten (Nägele \& Schneitter, 2016). Die Aufgaben können vielfältig sein, z. B. in der Aufarbeitung schulischer Inhalte zur Vorbereitung auf die Berufsfachschule, Vorbereitungskurse oder die Suche nach zusätzlichen Infomationen zur beruflichen Grundbildung.

Sich zu orientieren, zu bewerben und sich vorzubereiten beinhaltet jeweils mehrere einzelne Aufgaben. Eine Aufgabe, die sich bei jedem Thema wiederholt, ist die, dass Entscheidungen gefällt werden müssen. Auf einer analytischen Ebene können Bildungsentscheidungen durch Wert-Erwartungstheorien relativ verlässlich vorhergesagt werden. Es sind Modelle in der Tradition der Rational-choice-Theorien (Becker \& Lauterbach, 2016) oder motivationstheoretischer Theorien (Eccles, 2005). Solche Modelle berücksichtigen vor allem die kognitive Komponente der Entscheidung. Die Entscheidung basiert auf Werten, als Ausdruck der Wichtigkeit, Nützlichkeit, Interessantheit oder Kosten, und auf Erwartungen, als Ausdruck der Wahrscheinlichkeit, Ausbildungsziele zu erreichen. Die Berufswahl ist jedoch eine komplexe Entscheidung, die sich u. a. dadurch auszeichnet, dass nicht alle Optionen und Konsequenzen bekannt sind. Deshalb spielen auch emotionale Aspekte ein wichtige Rolle (Hellberg, 2009) und es sind teilweise „Bauchentscheidungen“" (Gigerenzer, 2015), bei denen auch der Zufall mitspielt (Mitchell, Levin, \& Krumboltz, 1999). Die Wahl der Ausbildung, des Berufsfelds oder Berufs kann also zufällig, ungeplant, emotional und „aus dem Bauch heraus“ erfolgen. Eine weitere Entscheidung steht nach der Bewerbung und allfälligen Selektion an. Denn es entscheidet nicht nur der Lehrbetrieb oder die Ausbildungsinstitution über die Aufnahme eines Jugendlichen. Auch die Jugendlichen selber entscheiden, ob sie von der Zulassung oder Zusage Gebrauch machen möchten. 


\section{Themen und Aufgaben auf der Sekundarstufe II}

Die Sekundarstufe II ist geprägt durch die Themen Ankommen, Lernen und Leisten sowie Weiterkommen (Abbildung 2). Das Ankommen umfasst die Begrüssung am neuen Ausbildungsort. Die Begrüssung umfasst idealerweise drei Elemente: informieren, willkommen heissen und anleiten (Klein \& Polin, 2012). Die Begrüssung der neuen Lernenden ist in der Praxis sehr unterschiedlich. Während z. B. einige Lernende von den Mitarbeiter/innen begrüsst werden, ein kleines Willkommensgeschenk erhalten oder eine Einführungswoche besuchen, sind andere Lernende mit der Situation konfrontiert, dass ihre Betreuungsperson eben noch in den Ferien weilt und niemand für sie zuständig ist. Dies sind Beobachtungen von Einzelfällen aus Interviews mit Lernenden, da eine systematische Übersicht der Strategien der Begrüssung neuer Lernender und deren Auswirkung auf den Verlauf der Ausbildung unseres Wissens nicht vorliegt. Aus der Forschung zur Einführung neuer Mitarbeiter/innen weiss man, dass eine gute Begrüssung und Einführung für einen guten Einstieg und Verlauf wichtig sind (Perrot et al., 2014).

Das Lernen umfasst fachliches Lernen und Sozialisationsprozesse. Ziel ist es, die im jeweiligen Kontext geforderte Leistung erbringen zu können. Hier besteht in der Berufsbildung ein wichtiger Unterschied im Vergleich zur Integration neuer Mitarbeiter/innen in einem Betrieb. Von neuen Mitarbeiter/innen wird erwartet, dass sie sich sozial schnell integrieren und sofort leistungsfähig sind. Bei Lernenden scheint jedoch die soziale Integration zuerst wichtiger zu sein als die fachliche Leistungsfähigkeit (Solinger, van Olffen, Roe, \& Hofmans, 2013).

Eine erfolgreiche soziale Integration erleichtert das Weiterkommen in der Ausbildung und auf der individuellen Laufbahn. Personen, die ausgeschlossen sind oder werden, lernen weniger und haben längerfristig schlechtere Entwicklungsmöglichkeiten, was sich exemplarisch im Fall von Mobbing als Form eines systematischen Ausschlusses aus einer Gruppe zeigt (Lereya, Copeland, Costello, \& Wolke, 2015; McDougall \& Vaillancourt, 2015). Deshalb bildet die soziale Integration eine wesentliche Voraussetzung für das Weiterkommen (Crosnoe, 2011; Wenger et al., 2002).

Anzukommen, zu lernen, zu leisten und weiterzukommen, umfasst mehrere Aufgaben. Eine wichtige Aufgabe ist, nach Beginn der Ausbildung die antizipierte Passung zu überprüfen und sich an die neue schulische und/oder betriebliche Ausbildungssituation anzupassen. Die bedeutet auch, die eigenen Laufbahnziele kontinuierlich zu überprüfen (Hirschi, 2009). Für Jugendliche in der Allgemeinbildung sind die Anpassungen beim Übergang in die Sekundarstufe II kleiner als für Lernende in der Berufsbildung, die ihre Rolle in einem hierarchisch strukturierten Betrieb finden müssen. Diese Anpassung verläuft für die meisten Jugendlichen 
jedoch ohne Schwierigkeiten (Nägele \& Neuenschwander, 2015). Im Vergleich zu den Jugendlichen in der Allgemeinbildung steigt die Zufriedenheit mit der Ausbildung bei den Jugendlichen in der Berufsbildung beim Eintritt sogar stärker an (Neuenschwander et al., 2012) und bleibt während der gesamten Ausbildung höher als bei den Jugendlichen in der Allgemeinbildung (Stalder, 2003). Während über die Anpassung der Jugendlichen in der Allgemeinbildung (Gymnasium, Fach-, Wirtschaftsmittelschule) kaum systematisch geforscht wird, liegen über Anpassungsprozesse in der beruflichen Grundbildung einige Forschungsarbeiten vor. Es gibt Hinweise, dass eine schlechte Anpassung zu Beginn der beruflichen Grundbildung das Risiko eines nicht-regulären Verlaufs der Ausbildung stark erhöht (Berweger, Krattenmacher, Salzmann, \& Schönenberger, 2013). Da sich Schüler/ innen auf der Sekundarstufe I vor allem mit der Wahl des Berufs auseinandersetzen, sind viele nur ungenügend über den Betrieb informiert (Stalder \& Schmid, 2016). Dadurch wird die Anpassung schwieriger und kann so bei diesen Jugendlichen zu einer geringen wahrgenommenen Passung führen als bei Jugendlichen, die sich bereits früher und bewusster mit der Betriebswahl auseinandergesetzt haben (Sluss, Ashforth, \& Gibson, 2012). Wenn die Erwartungen der Jugendlichen oder aber der Ausbildungsbetriebe nicht erfüllt werden, steigt das Risiko, dass der Anpassungsprozess negativ verläuft (Taris, Feij, \& Capel, 2006), womit auch das Risiko steigt, dass Lehrverträge aufgelöst werden (Stalder \& Schmid, 2016). Dies führt zur Notwendigkeit, diese Anpassungsprozesse intensiver und bewusster zu begleiten. Die Verantwortung dafür liegt bei den Betrieben und den Jugendlichen, da schulische Faktoren - also das, was die Jugendlichen mitgebracht haben - nur einen geringen direkten Einfluss auf die Anpassungsprozesse haben (Nägele \& Stalder, 2017; Stalder \& Carigiet Reinhard, 2014).

\subsection{Akteurinnen und Akteure}

Wichtige Akteurinnen und Akteure sind neben den Jugendlichen insbesondere deren Eltern, die Lehrpersonen, die Berufs-, Studien und Laufbahnberatung und die Berufsbildner/innen mit je spezifischen Rollen (SBFI, 2015). Diese Akteurinnen und Akteure stehen in einem mehr oder weniger konflikthaften Aushandlungsprozess, wobei durch eine kontinuierliche Koordination Lösungen gefunden werden (Wettstein et al., 2014). Wichtige Akteurinnen und Akteure sind in Abbildung 2 aufgeführt.

Die Eltern haben grundsätzlich einen grossen Einfluss auf die Wahl der weiterführenden Ausbildung (Rollett, 2014). Der Einfluss ist jedoch besonders gross bei beim Orientieren und der damit verbundenen Entscheidung für eine Ausbildung, 
ein Berufsfeld und die Ausbildungsinstitution (Beierle, 2013). Der starke Einfluss der Eltern auf die Berufswahl wird immer wieder dokumentiert (Neuenschwander, 2008; Neuenschwander et al., 2012). Eltern können in Ausübung ihrer elterlichen Gewalt direkt in die Laufbahnplanung ihrer Kinder eingreifen und steuern durch kontinuierliche Rückmeldungen, explizit formulierte Erwartungen und nicht direkt ausgesprochene (implizite) Erwartungen den Verlauf der Berufswahl. Auf der Sekundarstufe II verlieren die Eltern an Einfluss.

Die Lehrpersonen und die Berufs-, Studien- und Laufbahnberatung stossen die Schul- und Berufsorientierung oft an. Beim Thema Orientieren führen sie die Schüler/innen an Informationen zur Schul- und Berufswahl heran und erarbeiten diese mit ihnen. Beim Thema Bewerben bieten Schulen Trainings und Unterstützung an. Zudem haben manche Schulen Konzepte entwickelt, die Jugendliche beim Vorbereiten gezielt unterstützen, indem z. B. ein projektartiger Unterricht erfolgt.

Die Berufsbildner/innen sind nicht nur bei der Bewerbung und Selektion für eine Lehrstelle ausschlaggebend, sondern beeinflussen auch, ob sich Jugendliche für eine Allgemeinbildung oder eine Berufsbildung entscheiden. Dies gilt zum Beispiel dann, wenn sie signalisieren, dass sie keine Lehre mit Berufsmaturität unterstützen und so potentielle Lernende früh abweisen (Nägele, 2013). Mit Beginn der beruflichen Grundbildung verändert sich die Rolle der Berufsbildner/innen deutlich. Sie sind für die Begrüssung der Lernenden verantwortlich und beeinflussen den Anpassungsprozess durch gezielte Förderung und Unterstützung. Betriebe gestalten die Lernmöglichkeiten am Arbeitsplatz (Stalder \& Carigiet Reinhard, 2014) nicht zuletzt durch Feedback und die Entwicklung einer wertschätzenden Beziehung von Lernenden und Ausbildner/innen (Nägele \& Neuenschwander, 2016). Das Arbeitsmodell verweist darauf, dass verschiedene Akteurinnen und Akteure zu unterschiedlichen Zeitpunkten, zu verschiedenen Themen und Aufgaben für die Jugendlichen wichtig sind.

\section{$5 \quad$ Übergeordnete Prozesse}

\subsection{Passung}

Es ist bedeutsam, dass Schüler/innen die zukünftige Ausbildung auf der Sekundarstufe II als für sich passend wahrnehmen (Düggeli \& Neuenschwander, 2015). Dabei kann sich eine Person an die Organisation oder die Organisation an die Person und ihre Bedürfnisse anpassen (Kammeyer-Mueller, 2007). Während auf der Sekundarstufe I eine Anpassung der Schule an die Lernerfordernisse der 
Schüler/innen im Sinne der Stage-environment-fit-Theorien diskutiert wird, ist die Anpassungsleistung zu Beginn der beruflichen Grundbildung vornehmlich durch die Lernenden zu leisten (Masdonati, 2010; Masdonati \& Lamamra, 2009), ohne dabei die wichtige Rolle der Betriebe bei der Einführung der Lernenden zu vergessen.

\subsection{Soziale Integration}

Die soziale Integration ist für das Wohlbefinden und die Zufriedenheit der Jugendlichen wichtig, darüber hinaus aber auch eine Voraussetzung für erfolgreiches Lernen. Dies zeigt sich besonders deutlich in der betrieblichen Ausbildung, wo Lernende darauf angewiesen sind, Informationen und Feedback von ihren Arbeitskolleg/ innen zu erhalten (Billett, 2008). Soziale Integration setzt soziale Kompetenzen voraus. Soziale Kompetenzen helfen den Zugang zur Gruppe zu regulieren (Nägele, 2015). Soziale Kompetenzen als überfachliche Kompetenzen werden oft als sehr wichtige Kompetenzen im Übergang von der Schule in die Ausbildung auf Sekundarstufe II angesehen (Neuenschwander, 2012), auch wenn deren direkter Transfer zwischen der Volksschule und Berufsbildung fraglich ist (Nägele \& Stalder, 2017). So hängt die soziale Integration in der beruflichen Grundbildung nicht direkt von der Einschätzung der sozialen Kompetenz in der obligatorischen Schule ab, sehr wohl aber von Persönlichkeitsmerkmalen wie z. B. einer hohen Gewissenhaftigkeit (Nägele \& Neuenschwander, 2016). Dieser vermeintliche Widerspruch zwischen der hohen Bedeutung sozialer Kompetenzen (Neuenschwander \& Nägele, 2014) und der fehlenden direkten Transferierbarkeit kann damit erklärt werden, dass soziale Kompetenz nicht nur individuelle Kompetenzen sind, sondern sehr stark durch die Situation, andere Personen und den Betrieb bestimmt ist (Kanning, 2005). Soziale Kompetenzen in der Schule, bei der Bewerbung und beim Eintritt in die neue Ausbildung beziehen sich zudem auf unterschiedliche Kontexte.

\section{Zusammenfassung}

Das Arbeitsmodell von der obligatorischen Schule in die Berufsbildung in Abbildung 2 betrachtet die Zeit vor und nach dem Übergang von der Sekundarstufe I in die Sekundarstufe II. Die gezielte Auseinandersetzung mit den im Modell benannten Themen und Aufgaben und der damit verbundene Erwerb von Wissen, Fertigkeiten, Fähigkeiten und Einstellungen dürfte den weiteren Verlauf der Laufbahn genauso stark prägen wie das Ereignis des Übertritts an sich. Die Themen können je nach 
Verlauf des Übergangs und auch später bei der Auseinandersetzung mit der weiteren Ausbildung oder Weiterbildung immer wieder aufgegriffen werden. Anders als in Phasenmodellen wird dieses Wieder-Aufgreifen nicht als Zurückgehen oder als Rückschritt aufgefasst.

Das Arbeitsmodell kann genutzt werden, um exemplarisch am Übergang Sekundarstufe I - Sekundarstufe II wichtige Themen und Aufgaben darzustellen und zu systematisieren, die mit Blick auf eine lebenslange Karriereentwicklung immer wieder aufgenommen werden müssen. Auf der Sekundarstufe I sind es die Themen Orientieren, Bewerben und Vorbereiten, auf der Sekundarstude II Ankommen, Lernen - Leisten und Weiterkommen. Das Arbeitsmodell von der obligatorischen Schule in die Berufsbildung benennt darüber hinaus auch zentrale Akteurinnen und Akteure (z. B. Eltern, Kolleg/innen, Lehrpersonen, Berufs-, Studien- und Laufbahnberater/innen, Berufsbildner/innen), die den Übergang mitgestalten und je nach Zeitpunkt, Thema und Aufgabe eine andere Rolle einnehmen.

Wichtige dem Laufbahnprozess übergeordnete Prozesse sind das Finden einer Passung und die soziale Integration. Passung wird als individueller, subjektiver Ausdruck der wahrgenommenen Übereinstimmung von Person und ihrem schulischen und beruflichen Umfeld verstanden. Die soziale Integration ist eine Voraussetzung für die Entwicklung der Laufbahn. Je nach Thema und Aufgabe sind weitere Aufgaben und Akteurinnen und Akteure sowie theoretische Ansätze in das Modell zu integrieren, welche die lokale Dynamik im Gesamtkontext erklären können.

\section{Literatur}

Becker, R., \& Lauterbach, W. (2016). Bildung als Privileg. Erklärungen und Befunde zu den Ursachen der Bildungsungleichheit. Wiesbaden, D: Springer VS.

Beierle, S. (2013). Die Rolle von Peers, Neuen Medien und Online-Communitys bei der Berufsorientierung. München, D: Deutsches Jugendinstitut.

Berweger, S., Krattenmacher, S., Salzmann, P., \& Schönenberger, S. (2013). LiSa. Lernende im Spannungsfeld von Ausbildungserwartungen, Ausbildungsrealität und erfolgreicher Erstausbildung. St. Gallen, CH: Pädagogische Hochschule St. Gallen, Institut Professionsforschung und Kompetenzentwicklung.

Billett, S. (2008). Learning throughout working life: A relational interdependence between personal and social agency. British Journal of Educational Studies, 56, 39-58.

Carver, C. S., \& Scheier, M. F. (2002). Control processes and self-organization as complementary principles underlying behavior. Personality and Social Psychology Review, 304-315.

Crosnoe, R. (2011). Fitting in, standing out. Cambridge, NY: Cambridge University Press. 
D-EDK. (2015). Lehrplan 21. Luzern, CH: Deutschschweizer Erziehungsdirektoren-Konferenz (D-EDK).

Dubs, R. (2006). An appraisal of the Swiss vocational education and training system. Bern, $\mathrm{CH}$ : Huber AG.

Düggeli, A., \& Neuenschwander, M. P. (2015). Entscheidungsprozesse und Passungswahrnehmung. In K. Häfeli, M.P. Neuenschwander, S. Schumann (Hrsg.), Berufliche Passagen im Lebenslauf (S. 219-241). Wiesbaden, D: Springer Fachmedien. http://doi. org/10.1007/978-3-658-10094-0_9

Eccles, J. S. (2005). Subjective task value and the Eccles et al. model of achievement-related choices. In A. J. Elliot \& C. S. Dweck (Eds.), Handbook of competence and motivation (pp. 105-121). New York, NY: The Guilford Press.

Edwards, J. R., \& Shipp, A. J. (2007). The relationship between person-environment fit and outcomes: An integrative theoretical framework. In C. Ostroff \& T. A. Judge (Eds.), Perspectives on organizational fit (pp.209-258). New York, NY: Taylor \& Francis Group, LLC.

Fugate, M., Kinicki, A. J., \& Ashforth, B. E. (2004). Employability: A psycho-social construct, its dimensions, and applications. Journal of Vocational Behavior, 65, 14-38.

Gati, I., \& Tal, S. (2008). Decision-making models and career guidance. In J. A. Athanasou \& R. Van Esbroeck (Eds.), International Handbook of Career Guidance (pp. 157-185). Dordrecht, NL: Springer Verlag Netherlands.

Gigerenzer, G. (2015). Bauchentscheidungen: Die Intelligenz des Unbewussten und die Macht der Intuition. München: GoldmannVerlag.

Gonon, P. (2013). Federal vocational baccalaureate: The Swiss way of "hybridity". In T. Deissinger, J. Aff, A. Fuller \& C.H. Joergensen, Hybrid qualifications: Structures and problems in the context of European VET policy (pp.181-196). Bern: Peter Lang.

Gottfredson, L. S. (2002). Gottfredson's theory of circumscription, compromise, and self-creation. In D. Brown (Ed.), Career choice and development (4 ed., pp. 85-148). New York: Wiley \& Sons.

Hellberg, B.-M. (2009). Entscheidungsfindung bei der Berufswahl. Prozessmodell der Emotionen und Kognitionen. Wiesbaden, D: VS Verlag für Sozialwissenschaften.

Herzog, W., Neuenschwander, M. P., \& Wannack, E. (2001). Berufswahlprozess bei Jugendlichen. Bern, CH: Universität Bern, Institut für Pädagogik und Schulpädagogik, Abteilung Pädagogische Psychologie.

Hirschi, A. (2009). Career adaptability development in adolescence: Multiple predictors and effect on sense of power and life satisfaction. Journal of Vocational Behavior, 74, 145-155.

Hupka-Brunner, S., Scharenberg, K., Meyer, T., \& Müller, B. (2015). Leistung oder soziale Herkunft? In K. Häfeli, M.P. Neuenschwander, S. Schumann (Hrsg.), Berufliche Passagen im Lebenslauf(S. 243-275). Wiesbaden, D: Springer Fachmedien Wiesbaden. http://doi. org/10.1007/978-3-658-10094-0_10

Imdorf, C. (2007). Lehrlingsselektion in KMU. Freiburg, CH: Heilpädagogisches Institut der Universität Freiburg.

Jungo, D., \& Egloff, E. (2015). Berufswahl-Tagebuch. Bern, CH: Schulverlag plus.

Kammeyer-Mueller, J. D. (2007). The dynamics of newcomer adjustment: dispositions, context, interaction, and fit. In C. Ostroff \& T. A. Judge (Eds.), Perspectives on organizational fit (S. 99-122). New York, NY: Taylor \& Francis Group, LLC.

Kanning, U. P. (2005). Soziale Kompetenzen: Entstehung, Diagnose und Förderung. Göttingen, D: Hogrefe. 
Klein, H. J., \& Polin, B. (2012). Are organizations on board with best practices onboarding? In C. R. Wanberg (Ed.), The Oxford handbook of organizational socialization (pp. 267-287). New York, NY: Oxford University Press.

Kristof-Brown, A. L., Zimmerman, R. D., \& Johnson, E. C. (2005). Consequences of individuals' fit at work: A meta-analysis of person-job, person-organization, person-group, and person-supervisor fit. Personnel Psychology, 58, 281-342.

Lent, R. W., Brown, S. D., \& Hackett, G. (2002). Social cognitive career theory. In D. Brown (Ed.), Career choice and development (pp. 255-311). San Francisco, CA: Jossey-Bass.

Lereya, S. T., Copeland, W. E., Costello, E. J., \& Wolke, D. (2015). Adult mental health consequences of peer bullying and maltreatment in childhood: Two cohorts in two countries. Lancet Psychiatry, 1-8. http://doi.org/10.1016/S2215-0366(15)00165-0

Locke, E. A., \& Latham, G. P. (2002). Building a practically useful theory of goal setting and task motivation: A 35 year odyssey. American Psychologist, 57, 705-717.

Masdonati, J. (2010). The transition from school to vocational education and training: A theoretical model and transition support program. Journal of Employment Counseling, $47,20-29$.

Masdonati, J., \& Lamamra, N. (2009). La relation entre apprenti-e et personne formatrice au coeur de la transmission des savoirs en formation professionnelle. Schweizerische Zeitschrift für Bildungswissenschaften, 31(2), 335-353.

McDougall, P., \& Vaillancourt, T. (2015). Long-term adult outcomes of peer victimization in childhood and adolescence: Pathways to adjustment and maladjustment. American Psychologist, 70(4), 300-310. http://doi.org/10.1037/a0039174

Mitchell, K. E., Levin, A. S., \& Krumboltz, J. D. (1999). Planned happenstance: Constructing unexpected career opportunities. Journal of Counseling \& Development, 77(2), 115-124.

Müller, B., \& Schweri, J. (2009). Berufswechsel beim Übergang von der Lehre in den Arbeitsmarkt. Schweizerische Zeitschrift für Bildungswissenschaften, 31(2), 199-227.

Nägele, C. (2013). Wie sich Jugendliche für eine Lehre mit lehrbegleitender Berufsmaturität entscheiden. Studie im Auftrag des Kantons Luzern, Schlussbericht. Solothurn, CH: Fachhochschule Nordwestschweiz, Pädagogische Hochschule, Institut Forschung und Entwicklung, Zentrum Lernen und Sozialisation.

Nägele, C. (2015). Social integration as a key to successful learning at work. Presented at the Colloque scientifique de l'Institut de Psychologie du Travail et des Organisations IPTO, Neuchâtel, CH 27.11.

Nägele, C., \& Bierschenk, K. (2014). Höhere Berufsbildung im Bildungsraum Nordwestschweiz, Schlussbericht. Solothurn, CH: Fachhochschule Nordwestschweiz, Pädagogische Hochschule, Institut Forschung und Entwicklung, Zentrum Lernen und Sozialisation.

Nägele, C., \& Neuenschwander, M. P. (2015). Passt der Beruf zu mir? Determinanten und Konsequenzen wahrgenommener Passung mit dem Lehrberuf beim Übergang in die Berufsbildung. In K. Häfeli, M. P. Neuenschwander, \& S. Schumann (Hrsg.), Berufliche Passagen im Lebenslauf (S.49-74). Wiesbaden, D: Springer Fachmedien. http://doi. org/10.1007/978-3-658-10094-0_3

Nägele, C., \& Neuenschwander, M. P. (2016). Apprentice-trainer relationship and work group integration in the first months of an apprenticeship. Empirical Research in Vocational Education and Training, 8(1), 4. http://doi.org/10.1186/s40461-016-0030-3

Nägele, C., \& Schneitter, J. (2016). Schul- und Berufsorientierung in den Kantonen. Schlussbericht zu Handen der EDK. Solothurn, $\mathrm{CH}$ : Fachhochschule Nordwestschweiz, Pädagogische Hochschule, Institut Forschung und Entwicklung, Zentrum Lernen und Sozialisation. 
Nägele, C., \& Stalder, B. E. (2017). Competence and the need for transferable skills. In M. Mulder (Ed.), Competence-based vocational and professional education. Bridging the worlds of work and education (pp. 739-753). Cham, CH: Springer International Publishing.

Neuenschwander, M. P. (2008). Elternunterstützung im Berufswahlprozess. In D. Läge, A. Hirschi (Hrsg.), Berufliche Übergänge. Psychologische Grundlagen der Berufs-, Studienund Laufbahnberatung (S.135-153). Zürich, CH: Lit Verlag.

Neuenschwander, M. P., \& Nägele, C. (2014). So gelingt der Start in die Berufslehre. Panorama, 5(2014), 5.

Neuenschwander, M. P., Gerber, M., Frank, N., \& Rottermann, B. (2012). Schule und Beruf: Wege in die Erwerbstätigkeit. Wiesbaden, D: VS Verlag für Sozialwissenschaften.

Parsons, F. (1909). Choosing a Vocation. Boston, MA: Houghton Mifflin Company.

Perrot, S., Bauer, T. N., Abonneau, D., Campoy, E., Erdogan, B., \& Liden, R. C. (2014). Organizational socialization tactics and newcomer adjustment: The moderating role of perceived organizational support. Group \& Organization Management, 39(3), 247-273. http://doi.org/10.1177/1059601114535469

Rollett, B. (2014). Eltern als Akteure in Bildungsprozessen aus entwicklungspsychologischer Perspektive. Erziehung und Unterricht, (7-8), 604-613.

SBFI. (2015). Berufsbildung in der Schweiz. Fakten und Zahlen 2015.

Sluss, D. M., Ashforth, B. E., \& Gibson, K. R. (2012). The search for meaning in (new) work: Task significance and newcomer plasticity. Journal of Vocational Behavior, 81, 199-208.

Solinger, O. N., van Olffen, W., Roe, R. A., \& Hofmans, J. (2013). On becoming (un)committed: A taxonomy and test of newcomer onboarding scenarios. Organization Science, 24(6), 1640-1661. http://doi.org/10.1287/orsc.1120.0818

Stalder, B. E. (2000). Gesucht wird... Rekrutierung und Selektion von Lehrlingen im Kanton Bern. Bern, CH: Amt für Bildungsforschung, Erziehungsdirektion des Kantons Bern.

Stalder, B. E. (2002). Was ist ein guter Lehrling? Panorama, 1, 38-39.

Stalder, B. E., \& Carigiet, T. (2014). Ausbildungsqualität aus Sicht von Lernenden und Betrieben in der Schweiz. In M. Fischer (Hrsg.), Qualität in der Berufsbildung. Anspruch und Wirklichkeit. Bericht zur beruflichen Bildung (S. 97-118). Bielefeld, D: W. Bertelsmann Verlag.

Stalder, B. E., \& Nägele, C. (2015). Berufliche Identität, Commitment und Engagement. In M. Fischer, F. Rauner, \& Z. Zhao (Hrsg.), Kompetenzdiagnostik in der beruflichen Bildung - Methoden zum Erfassen und Entwickeln beruflicher Kompetenz (S. 259-273). Münster: LIT Verlag.

Stalder, B. E., \& Schmid, E. (2016). Lehrvertragsauflösung und Ausbildungserfolg - kein Widerspruch. Bern, $\mathrm{CH}$ : hep verlag.

Stamm, M., Holzinger-Neulinger, M., Suter, P., \& Stroezel, H. (2007). Die Zukunft verlieren? Schulabbrecher in der Schweiz.

Stricker, C., \& Stalder, B. E. (2009). Ausbildungsqualität an der Schwelle zum Arbeitsmarkt. Betriebliche, schulische und überbetriebliche Ausbildung und ihre Akteure. Eine repräsentative Befragung von Betrieben des Kantons Bern. Bern, CH.

Stringer, K., Kerpelman, J., \& Skorikov, V. (2011). Career preparation: A longitudinal, process-oriented examination. Journal of Vocational Behavior, 79(1), 158-169. http://doi. org/10.1016/j.jvb.2010.12.012

Taris, T. W., Feij, J. A., \& Capel, S. (2006). Great expectations - and what comes of it: The effects of unmet expectations on work motivation and outcomes among newcomers. International Journal of Selection and Assessment, 14, 256-268. 
Vondracek, F. W., Lerner, R. M., \& Schulenberg, J. E. (1986). Career Development: A life-span developmental approach. Hillsdale, NJ: Lawrence Erlbaum Associates, Inc., Publishers.

Wenger, E. (2008). Communities of practice. Learning, meaning, and identity. New York, NY: Cambridge University Press.

Wenger, E., McDermott, R., \& Snyder, W. M. (2002). A guide to managing knowledge. Cultivating communities of practice. Boston, MA: Business School Publishing.

Wettstein, E., Schmid, E., \& Gonon, P. (2014). Berufsbildung in der Schweiz. Formen, Strukturen, Akteure. Bern, $\mathrm{CH}$ : hep verlag.

Open Access Dieses Kapitel wird unter der Creative Commons Namensnennung 4.0 International Lizenz (http://creativecommons.org/licenses/by/4.0/deed.de) veröffentlicht, welche die Nutzung, Vervielfältigung, Bearbeitung, Verbreitung und Wiedergabe in jeglichem Medium und Format erlaubt, sofern Sie den/die ursprünglichen Autor(en) und die Quelle ordnungsgemäß nennen, einen Link zur Creative Commons Lizenz beifügen und angeben, ob Änderungen vorgenommen wurden.

Die in diesem Kapitel enthaltenen Bilder und sonstiges Drittmaterial unterliegen ebenfalls der genannten Creative Commons Lizenz, sofern sich aus der Abbildungslegende nichts anderes ergibt. Sofern das betreffende Material nicht unter der genannten Creative Commons Lizenz steht und die betreffende Handlung nicht nach gesetzlichen Vorschriften erlaubt ist, ist für die oben aufgeführten Weiterverwendungen des Materials die Einwilligung des jeweiligen Rechteinhabers einzuholen.

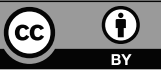


B

\section{Diachrone Verläufe in der Schule}




\section{Irreguläre Schullaufbahnen im Rahmen der obligatorischen Schule und Forschungsergebnisse zur Klassenwiederholung}

Gérard Bless

\section{Zusammenfassung}

Dem Bildungssystem stehen zur Erfüllung seiner Allokationsfunktion zahlreiche Varianten von schulischen Selektionsmassnahmen zur Verfügung. Im schweizerischen Bildungssystem werden diese auch extensiv genutzt, was unweigerlich Folgen für die Schullaufbahnen der Schülerinnen und Schüler hat. Zur Veranschaulichung werden Beispiele bildungsstatistischer Analysen auf der Grundlage von Daten des Bundesamtes für Statistik (Schweiz) pro Schuljahr sowie auf der Grundlage von Individualdaten und somit von echten Laufbahnen beschrieben. Dabei wird für künftige Analysen mit Individualdaten auf Bundes- und Kantonsebene plädiert, da diese das Ausmass der feststellbaren Phänomene prägnanter beschreiben. Auf nationaler Ebene ist zu erwarten, dass spätestens 2026 Kohorten mit vollständigen individuellen Datensätzen zur Verfügung stehen werden. Weiter wird mit einem kurzen Forschungsüberblick über die Wirksamkeit der Klassenwiederholung aufgezeigt, dass sich diese, im Widerspruch zu ihrer häufigen Anordnung, nicht im erwünschten Masse als wirksam erweist. Während über die Effekte der Aussonderung ebenfalls zahlreiche empirische Studien vorliegen, fehlen Forschungsarbeiten, welche andere Selektionsmassnahmen unter den Bedingungen des Schweizerischen Bildungssystems untersuchen.

\section{Schlagworte}

Schulische Selektion, Schullaufbahn, Bildungsverläufe, Bildungsstatistik, Wirksamkeit der Klassenwiederholung 


\section{Schulische Selektionsmassnahmen}

Die Betrachtung von Schullaufbahnen aus dem Blickwinkel der Sonderpädagogik führt unweigerlich zum Thema der schulischen Selektion. Dem Bildungssystem stehen hierzu vielfältige Varianten zur Verfügung, welche in der Regel mit bestem Wissen und Gewissen zum Wohle des betroffenen Kindes und manchmal auch zur Herstellung eines reibungsloseren Funktionierens der Regelschule verordnet werden. Das Bildungssystem strebt damit eine möglichst geringe Heterogenität in Bezug auf die schulischen Leistungen der Schülerschaft an. Begründet wird dies häufig mit der Annahme, dass in leistungshomogenen Lerngruppen bessere Lernfortschritte zu erzielen seien. Davon sind insbesondere viele Schulakteure im Feld überzeugt. Zahlreiche empirische Befunde stellen dies jedoch in Frage.

Leider wird bei Entscheidungen dieser Art häufig vergessen, dass die meisten Selektionsmassnahmen auch Nebenwirkungen haben. Dazu zählen insbesondere jene, die die Schullaufbahn der betroffenen Schülerinnen und Schüler verlängern, wie beispielsweise der verspätete Eintritt in den Kindergarten, in die Primar- oder Sekundarstufe I, die Klassenwiederholung, negative und positive Typenwechsel auf der Sekundarstufe I und schliesslich auch die Aussonderung in eine Sonderklasse oder in eine Sonderschule. Die genannten Selektionsmassnahmen können die Schullaufbahnen und im weiteren Verlauf auch die Berufslaufbahnen negativ vorstrukturieren (vgl. Gomolla \& Radke, 2009). Wer beispielsweise zu Beginn der Primarschule eine Klasse wiederholt, hat im späteren Schulverlauf ein erhöhtes Risiko, eine Klasse erneut zu wiederholen, ausgesondert zu werden oder beim Übertritt in die Sekundarstufe I Klassen mit geringeren Ansprüchen zugeteilt zu werden. Im Gegensatz hierzu werden mit dem Überspringen einer Klasse in der Regel die Schullaufbahnen verkürzt oder mit dem Schulabbruch aufgrund des Alters, bevor alle Klassenstufen durchlaufen worden sind, zumindest nicht weiter verlängert.

In der Regel werden Selektionsmassnahmen von den Schulakteuren und somit letztendlich vom Bildungssystem verordnet, wobei es auch Ausnahmen gibt. Beim verspäteten Eintritt in den Kindergarten, beim Wechsel in eine Privatschule, beim Abbruch der Schule aufgrund des Alters und etwas seltener bei der Klassenwiederholung sind manchmal auch Eltern (mit oder ohne Beratung durch Schulakteure) für diese Entscheidung verantwortlich.

Interessant ist zudem, dass zur Erreichung einer möglichst geringen leistungsmässigen Heterogenität bedeutsame andere Heterogenitäten in Kauf genommen werden, welche auf Seiten der Schulakteure wenig Beachtung finden. Das folgende 
Beispiel $^{1}$ dient der Illustration solcher Nebenwirkungen (vgl. Tabelle 1). Verwendet wird eine relativ harmlos erscheinende Variable, und zwar das Alter der Schülerinnen und Schüler. Die Grundlage der Darstellung bilden die vom Bundesamt für Statistik publizierten Daten aller Schülerinnen und Schüler des Schuljahres 2013/14 der gesamten Schweiz. ${ }^{2}$ In der Spalte „Normalter“ wird pro Klassenstufe das Normalter gemäss Vorgaben des HarmoS-Konkordates angegeben. Die Spalte „Altersspanne“ gibt Auskunft über die Streuung des tatsächlichen Alters der Schülerinnen und Schüler pro Klassenstufe. In der Spalte „Mittelwert“ wird das durchschnittliche Alter der Kinder auf der entsprechenden Klassenstufe angegeben. Mit Fortschreiten der Schuljahre nähert sich das Durchschnittsalter der Kinder immer mehr der Obergrenze der vorgegebenen Altersnorm an. Das weist darauf hin, dass sich die Schullaufbahn zahlreicher Kinder verlängert. 10-jährige Kinder sollten sich aufgrund der Altersvorgaben in der Primarklasse $6 \mathrm{H}^{3}$ oder $7 \mathrm{H}$

Tab. 1 Alter der Schülerinnen und Schüler pro Klassenstufe im Schuljahr 2013/2014

\begin{tabular}{lccc}
\hline Klassenstufe nach HarmoS & Normalter & Altersspanne & Mittelwert \\
\hline KG $1 \mathrm{H}$ und $2 \mathrm{H}$ & 4 bis 6 & 3 bis 10 & 5.03 \\
\hline Prim $3 \mathrm{H}$ & 6 bis 7 & 5 bis 11 & 6.59 \\
\hline Prim $4 \mathrm{H}$ & 7 bis 8 & 5 bis 11 & 7.63 \\
\hline Prim $5 \mathrm{H}$ & 8 bis 9 & 7 bis 12 & 8.65 \\
\hline Prim $6 \mathrm{H}$ & 9 bis 10 & 7 bis 13 & 9.67 \\
\hline Prim $7 \mathrm{H}$ & 10 bis 11 & 8 bis 14 & 10.67 \\
\hline Prim $8 \mathrm{H}$ & 11 bis 12 & 9 bis 15 & 11.73 \\
\hline Sek-I $9 \mathrm{H}^{1}$ & 12 bis 13 & 10 bis 17 & 12.79 \\
\hline Sek-I $10 \mathrm{H}^{1}$ & 13 bis 14 & 10 bis 18 & 13.82 \\
\hline Sek-I $11 \mathrm{H}^{1}$ & 14 bis 15 & 12 bis 20 & 14.88 \\
\hline Besonderer Lehrplan & 4 bis 20 & 3 bis 29 & 11.18 \\
\hline
\end{tabular}

${ }^{1}$ Nur Schülerinnen und Schüler berücksichtigt, welche eindeutig der HarmoS-Stufe zugeordnet werden können.

1 Alle Beispiele beziehen sich auf die Schweiz. Information zum schweizerischen Bildungssystem unter www.edk.ch.

2 Die entsprechenden Daten wurden der Online-Datenbank des Bundesamtes für Statistik entnommen und vom Autor weiterverarbeitet: https://www.bfs.admin.ch/bfs/de/home/ statistiken/ bildung-wissenschaft/personen-ausbildung/ obligatorische-schule.html

3 Bezeichnung der Klassenstufen der obligatorischen Schule (Kindergarten, Primarschule und Sekundarstufe I) gemäss HarmoS-Konkordat: 1 . und 2. Kindergartenjahr $=1 \mathrm{H}$ und $2 \mathrm{H}, 1$. bis 6. Primarklasse $=3 \mathrm{H}$ bis $8 \mathrm{H}, 1$. bis 3 . Klasse der Sekundarstufe $\mathrm{I}=9 \mathrm{H}$ bis $11 \mathrm{H}$. 
befinden. Tatsächlich befinden sich diese Kinder aber in allen Klassenstufen der obligatorischen Schule, mit Ausnahme der letzten Klasse. Im Kindergarten sind die 10-Jährigen somit bis zu 7 Jahre älter als die jüngsten Kinder dieser Stufe, in der vorletzten Klasse (10H) bis zu 8 Jahre jünger als die ältesten Jugendlichen dieser Klassenstufe.

Offensichtlich gelingt die Organisation des Bildungssystems nach Jahrgangsklassen nicht. Es ist anzunehmen, dass die Altersunterschiede sowohl für die deutlich jüngeren als auch für die deutlich älteren Kinder nicht förderlich sind, zum Beispiel hinsichtlich der eigenen Identitätsentwicklung oder des Selbstwertgefühls. Zudem dürfte der Einstieg der Schulabgängerinnen und -abgänger in die Berufsausbildung im Falle einer zu starken Abweichung vom Normalter erschwert sein. Solche Nebenwirkungen der extensiven schulischen Selektion werden äusserst selten thematisiert.

Theoretisch betrachtet weisen die schulischen Selektionsmassnahmen einen direkten Bezug zur Allokationsfunktion der Schule auf, welche gemäss Fend (2006) nebst der Enkulturations-, der Qualifikations- und der Integrationsfunktion zu den vier grundlegenden Funktionen der Schule gehört. Schulische Selektion ist somit als Instrument des Bildungssystems zur Erfüllung ihrer Allokationsfunktion zu verstehen. Wie im vorliegenden Beitrag noch gezeigt wird, kann aber nicht davon ausgegangen werden, dass sie für das betroffene Kind, pädagogisch betrachtet, immer sinnvoll und zielführend ist. Gegenüber Selektionsentscheidungen, welche mit dem Argument des Kindeswohls begründet werden, scheint somit eine gewisse Skepsis angebracht zu sein. Einerseits muss das Bildungssystem die Allokationsfunktion erfüllen, und zu diesem Zwecke müssen die Schülerinnen und Schüler sortiert werden. Andererseits müssen häufig Argumente ins Feld geführt werden, damit solche Entscheidungen von den Eltern besser akzeptiert werden und die Schulakteure gleichzeitig ohne schlechtes Gewissen solche Entscheidungen fällen können.

\section{Bildungsstatistische Analysen}

Im Folgenden werden sowohl Analysen bildungsstatistischer Daten auf der Basis von Daten pro Schuljahr als auch Analysen von Schullaufbahnen auf der Grundlage von Individualdaten anhand von Beispielen dokumentiert. Die Beispiele werden aus der Perspektive der Sonderpädagogik ausgewählt und stehen in Bezug zu den schulischen Selektionsmassnahmen. 


\subsection{Analysen auf der Basis von Daten pro Schuljahr}

Wie in Tabelle 2 dargestellt, zählte die obligatorische Schule der Schweiz ${ }^{4}$ während des Schuljahres 2014/15 insgesamt 920'958 Kinder, wovon 31'270 eine Sonderklasse oder Sonderschule besuchten.

Die Aussonderungsquote beträgt somit 3.4\% und ist unterdessen im internationalen Vergleich unter den westlichen Industrienationen als durchschnittlich einzustufen. Die Gesamtzahl der ausgesonderten Schülerinnen und Schüler entspricht in etwa der gesamten Schülerpopulation der obligatorischen Schule des Kantons Basel Landschaft. In diesem Sinne handelt es sich um eine grosse Schülergruppe.

Tab. 2 Schülerinnen und Schüler im Schuljahr 2014/15

\begin{tabular}{lccc}
\hline $\begin{array}{l}\text { Obligatorische Schule } \\
\text { (Kindergarten, Primar, Sek I, } \\
\text { besonderer Lehrplan) }\end{array}$ & Regelklassen & $\begin{array}{l}\text { Sonderklassen und } \\
\text { Sonderschulen }\end{array}$ \\
\hline $\begin{array}{l}\text { Total } \\
\text { in \% }\end{array}$ & $920^{\prime} 958$ & $889^{\prime} 688$ & $31^{\prime} 270$ \\
\hline männlich & $474^{\prime} 244$ & $51.03 \%$ & $3.40 \%$ \\
weiblich & $446^{\prime} 714$ & $48.97 \%$ & $64.82 \%$ \\
\hline Schweizer & $677^{\prime} 895$ & $74.26 \%$ & $35.18 \%$ \\
Nicht-Schweizer & $243^{\prime} 063^{*}$ & $25.74 \%$ & $54.93 \%$ \\
\hline
\end{tabular}

*wovon bei 1'288 Kindern die Staatsangehörigkeit nicht bekannt ist

4 Alle Daten pro Schuljahr und für die gesamte Schweiz wurden der Online-Datenbank des Bundesamtes für Statistik entnommen und vom Autor weiterverarbeitet: https:// www.bfs.admin.ch/bfs/de/home/statistiken/bildung-wissenschaft/personen-ausbildung/ obligatorische-schule.html 


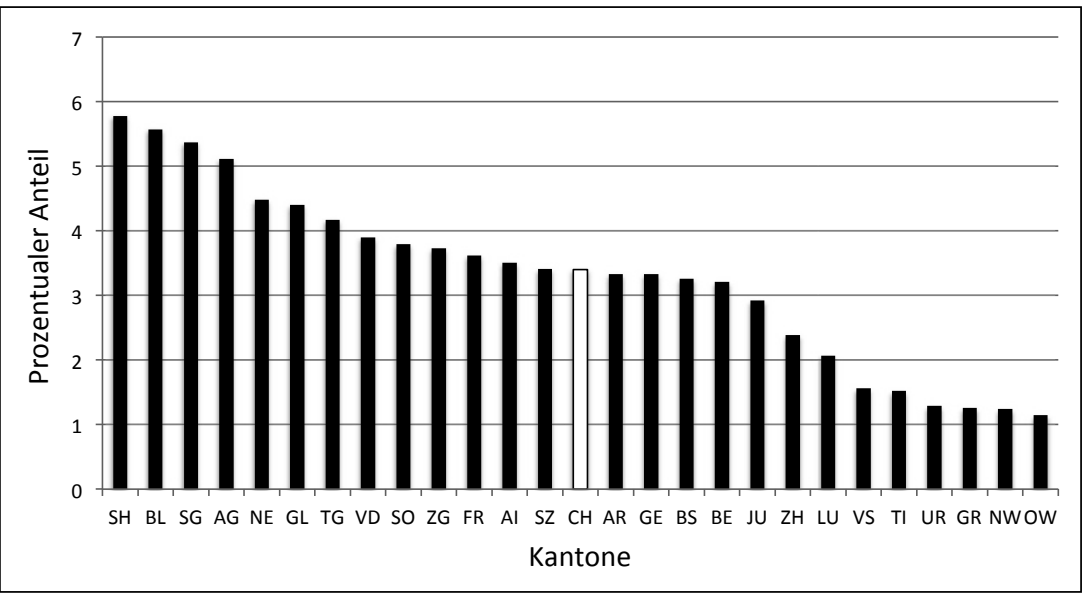

Abb. 1 Kantonale Unterschiede hinsichtlich der Aussonderungsquote im Schuljahr 2014/15

In Bezug auf die Aussonderungsquote gibt es enorme kantonale Differenzen (vgl. Abbildung 1). Die Aussonderungsquoten schwanken zwischen 1.15\% (Kanton Obwalden) und 5.78\% (Kanton Schaffhausen). Während im Kanton mit der niedrigsten Quote jedes 87. Kind ausgesondert wird, ist es in jenem mit der höchsten Quote jedes 17. Kind. Letzteres entspricht einer fünfmal höheren Aussonderungsquote. Die kantonalen Unterschiede weisen auf die jeweilige Bildungspolitik und die Umsetzung des Konkordates Sonderpädagogik der Kantone hin. Die rote Säule auf Abbildung 1 kennzeichnet die Aussonderungsquote für die gesamte Schweiz. Die Zahlen zeigen, dass die Bildungspolitik des Wohnortkantons die Schullaufbahnen von Kindern mit einem leichteren sonderpädagogischen Förderbedarf und damit die Chancengleichheit empfindlich beeinflussen kann. Vergleicht man die prozentualen Anteile der Geschlechter sowie der nationalen Herkunft in Sonderklassen und Sonderschulen mit jenen in Regelschulklassen (Tabelle 2), so ist in ersteren sowohl eine massive Übervertretung der Knaben als auch der Schülerinnen und Schüler ohne Schweizer Pass festzustellen. Als mögliche Erklärungen für die Überrepräsentation der Knaben gelten die biologische Fragilität des männlichen Geschlechts, das extrovertiertere Verhalten der Knaben und die nach wie vor höheren Leistungserwartungen an diese, insbesondere in Familien mit niedrigem sozioökonomischem Status. Als potentielle Erklärungen für die Überrepräsentation der Kinder ausländischer Herkunft werden häufig mangelnde 
Kenntnisse der Unterrichtssprache, kulturelle Eigenheiten, mangelnde familiäre Unterstützungsmöglichkeiten, ein niedriger sozioökonomischer Status, ungenügende Kenntnisse des Bildungssystems und Unterschätzung der Schulleistungen durch die Lehrpersonen angeführt. Die genannten potentiellen Ursachen vermögen jedoch die enorme Ausprägung der Überrepräsentationen nicht vollständig zu erklären.

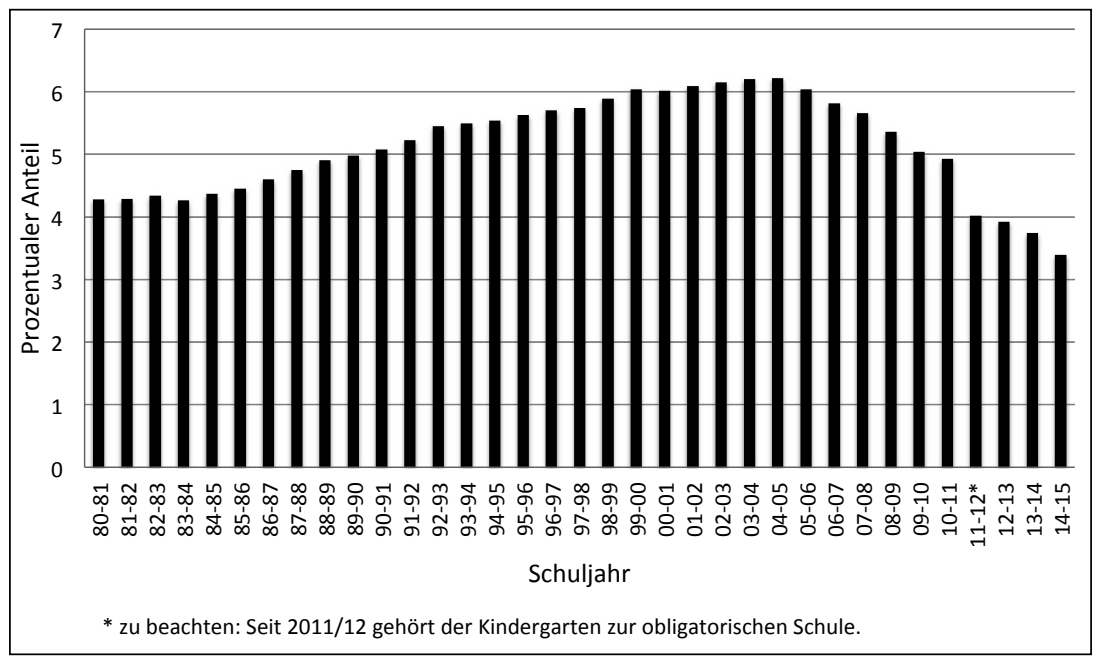

Abb. 2 Aussonderungsquote ab dem Schuljahr 1980/81

Es stellt sich die Frage, ob die festgestellten Phänomene stabil sind oder sich über die Zeit verändert haben. Hierfür ist die Analyse mehrerer Schuljahre erforderlich (Abbildung 2). Die Aussonderungsquote hat sich im Verlauf der letzten 35 Schuljahre beträchtlich verändert. Bis zum Schuljahr 2004/05 nahm die Aussonderungsquote jährlich zu, und zwar von $4.28 \%$ bis auf $6.22 \%$. Seither ist eine kontinuierliche Abnahme bis auf $3.40 \%$ im Schuljahr 2014/15 zu beobachten. Während im Schuljahr 2004/05 noch jedes 16. Kind ausgesondert wurde, war dies im Schuljahr 2014/15 nur noch für jedes 30. Kind der Fall.

Der Rückgang der Aussonderungsquote ist vorwiegend auf die vermehrte Integration von Kindern mit sonderpädagogischem Förderbedarf in Regelklassen und insbesondere auf die damit einhergehende Schliessung von Sonderklassen (Kleinklassen für Kinder mit Lernschwierigkeiten) zurückzuführen. Ein Rückgang der Aussonderungsquote gilt im Vergleich zur Quote integriert beschulter 
Schülerinnen und Schüler (Integrationsquote) als aussagekräftigeres Indiz für die tatsächliche Umsetzung integrativer Schulungsformen.

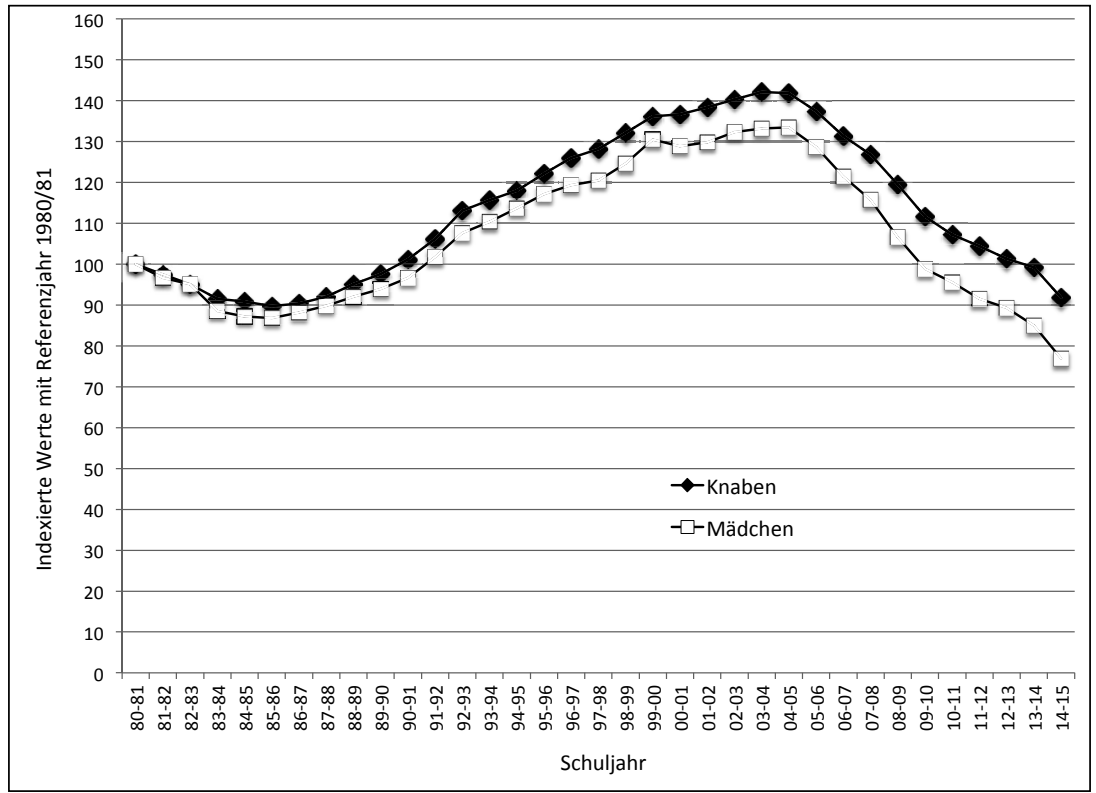

Abb. 3 Überrepräsentation von Knaben in Sonderklassen und Sonderschulen seit dem Schuljahr 1980/81

In Abbildung 3 ist die Entwicklung der Überrepräsentation von Knaben in Sonderklassen und Sonderschulen ab dem Schuljahr 1980/81 dargestellt. Die Graphik basiert auf indexierten Werten mit dem Referenzjahr 1980/81, das heisst, die absoluten Zahlen wurden im Referenzjahr künstlich auf jeweils $100 \%$ gesetzt. Ein Auseinanderdriften der Kurven ohne Überkreuzung ist gleichbedeutend mit der Akzentuierung eines Trends. Es ist ersichtlich, dass die bereits 1980/81 vorhandene Überrepräsentation der Knaben im Verlaufe der Zeit noch leicht zugenommen hat (Abbildung 3). 


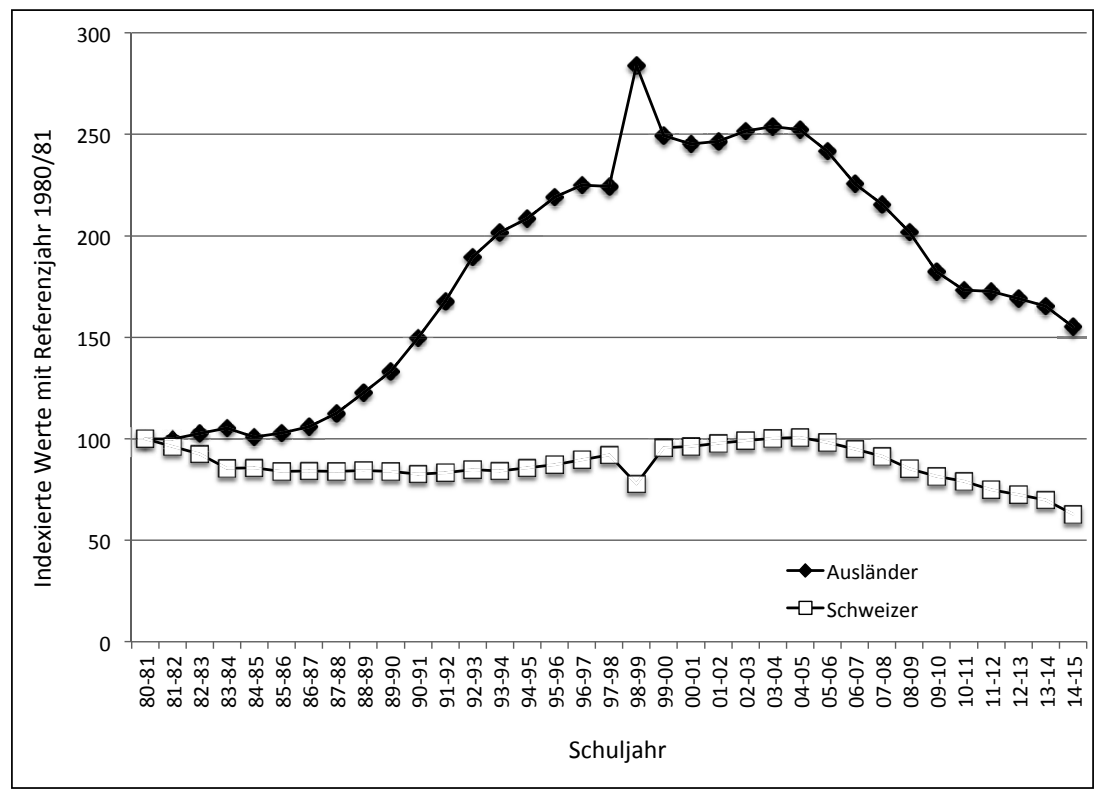

Abb. 4 Überrepräsentation von Kindern ausländischer Herkunft in Sonderklassen und Sonderschulen seit dem Schuljahr 1980/81

In Abbildung 4 ist die Entwicklung der Überrepräsentation von Kindern ausländischer Herkunft in Sonderklassen und Sonderschulen seit dem Schuljahr 1980/81 dargestellt. Das Darstellungsprinzip folgt jenem von Abbildung 3. Die bereits im Schuljahr 1980/81 bestehende Überrepräsentation von Kindern ausländischer Herkunft hat sich über die Zeit sehr stark verändert. Bis zum Schuljahr 2004/05 hat diese Überrepräsentation erheblich zugenommen. Anschliessend ist bis zum Schuljahr 2014/15 eine deutliche Abnahme zu verzeichnen. Dieses Auf und Ab der Überrepräsentation von ausländischen Kindern steht in engem Zusammenhang mit der Entwicklung der Aussonderungsquote (vgl. Abbildung 2). Die Aussonderung trifft überproportional Schülerinnen und Schüler ohne Schweizer Pass, und Schwankungen in der Aussonderungsquote sind vor allem auf Schwankungen in der Aussonderung dieser Schülergruppe zurückzuführen. ${ }^{5} \mathrm{Zu}$ beachten ist, dass

5 Die unerklärbare Spitze nach oben und nach unten im Schuljahr 1998/99 kann vernachlässigt werden, da diese mit grosser Wahrscheinlichkeit auf einen Kodierungsfehler in den Daten zurückzuführen ist. 
es sich bei den ausländischen Kindern mehrheitlich um Kinder handelt, die bereits in der Schweiz geboren wurden.

\subsection{Analysen auf der Basis von Individualdaten}

Wenngleich mit der Analyse der Daten pro Schuljahr interessante Phänomene beschrieben und einige Rückschlüsse auf die Bildungsverläufe gezogen werden können, verharmlosen sie in der Regel das Ausmass der jeweiligen Problematik. Als Beispiel sei hier die Repetentenquote (prozentualer Anteil der Schülerinnen und Schüler an der Gesamtschülerschaft der obligatorischen Schule, welche eine Klasse wiederholen) aufgeführt. Gesamtschweizerisch beträgt die Repetentenquote über die Jahre jeweils etwa $2.5 \%$, wobei auch hier grosse kantonale Unterschiede bestehen. Diese Quote scheint nicht besonders hoch zu sein. Betrachtet man jedoch individuelle Bildungsverläufe, so werden das Ausmass und die Dramatik dieser einen Selektionsmassnahme deutlich. Aus diesem Grunde wird sowohl für gesamtschweizerische als auch für kantonale Analysen von Bildungsverläufen plädiert, welche auf individuellen Längsschnittdaten und somit auf echten Schullaufbahnen basieren. Voraussetzung hierfür sind Datenbanken mit einem individuellen Schülercode pro Kind. Seit 2013 stehen dem Bundesamt für Statistik solche Daten zur Verfügung. Um Schullaufbahnen über die gesamte obligatorische Schule abzubilden, sind vollständige Datensätze erforderlich. Diese müssten spätestens 2026 vorhanden sein.

Im Folgenden werden echte Längsschnitte aus drei Kantonen (Freiburg, Neuenburg und Aargau) gezeigt, die als Anregung für ein solches Unterfangen für die gesamte Schweiz dienen könnten. Zunächst werden jeweils die Schullaufbahnen für eine Kohorte über die gesamte obligatorische Schule zusammengetragen und analysiert. Die Abbildungen 5, 6 und 7 zeigen jeweils auf, wie hoch pro Schuljahr der Anteil der Kinder ist, welche eine reguläre beziehungsweise eine abweichende Schullaufbahn aufweisen. In Tabelle 3 wird die Art der Abweichungen quantitativ beschrieben.

Abbildung 5 zeigt die Schullaufbahnen der Freiburger Kohorte für die Schuljahre 1992/93 bis 2001/02. Im Gesamtbild ist festzuhalten, dass bereits am Ende des Kindergartens nur $84.9 \%$ der Schülerinnen und Schüler im folgenden Schuljahr entsprechend einer regulären Schullaufbahn die 1. Klasse der Primarstufe besuchten. $15.1 \%$ hingegen wurden bereits im Kindergarten von einer ersten Selektionsmassnahme erfasst. Im Laufe der Schuljahre verkleinert sich der Anteil der Schülerinnen und Schüler mit einer regulären Schullaufbahn markant, sodass am Ende der obligatorischen Schule lediglich noch 52.8 \% die Schule regulär durchlaufen haben. Die übrigen Schülerinnen und Schüler weisen eine abweichende 
Schullaufbahn auf. Der grösste Schülerschwund ist zu Beginn und gegen Ende der Schulkarriere zu verzeichnen. Die Abnahme regulärer Schullaufbahnen ist zwischen der 5. Primarklasse und der 1. Klasse der Sekundarstufe I am geringsten. Dies ist im Zusammenhang mit dem Übertrittsverfahren von der Primar- in die Sekundarstufe I nachvollziehbar. Bemerkenswert ist, dass bereits ein Jahr nach der Übertrittsselektion ein grosses Bedürfnis nach weiterer Selektion besteht. Reguläre Laufbahnen nehmen damit in den letzten beiden Schuljahren nochmals massiv ab.

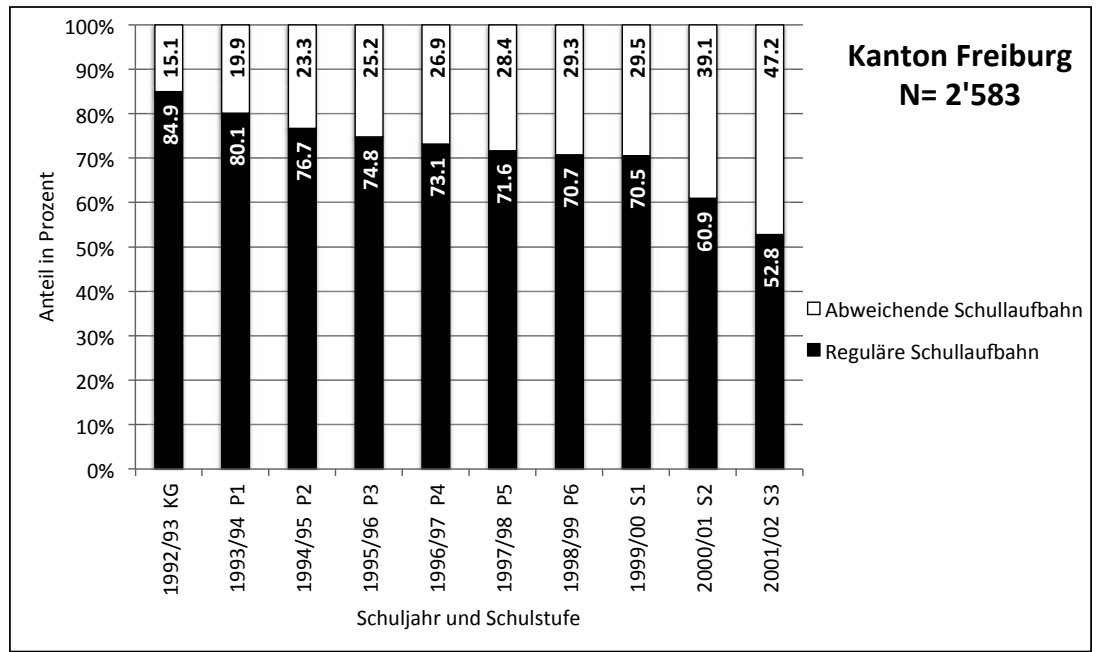

Abb. 5 Schullaufbahnen im Kanton Freiburg der Kohorte 1992/93 bis 2001/02

(Taminelli, 2007)

Abbildung 6 zeigt die Situation einer Kohorte im Kanton Neuenburg für die Schuljahre 1995/96 bis 2005/06. Hier gelingt 60.4\% der Schülerinnen und Schüler der obligatorischen Schule eine reguläre Schullaufbahn. Der Anteil abweichender Schullaufbahnen beträgt $39.6 \%$ und ist ebenfalls als sehr hoch einzuschätzen. 


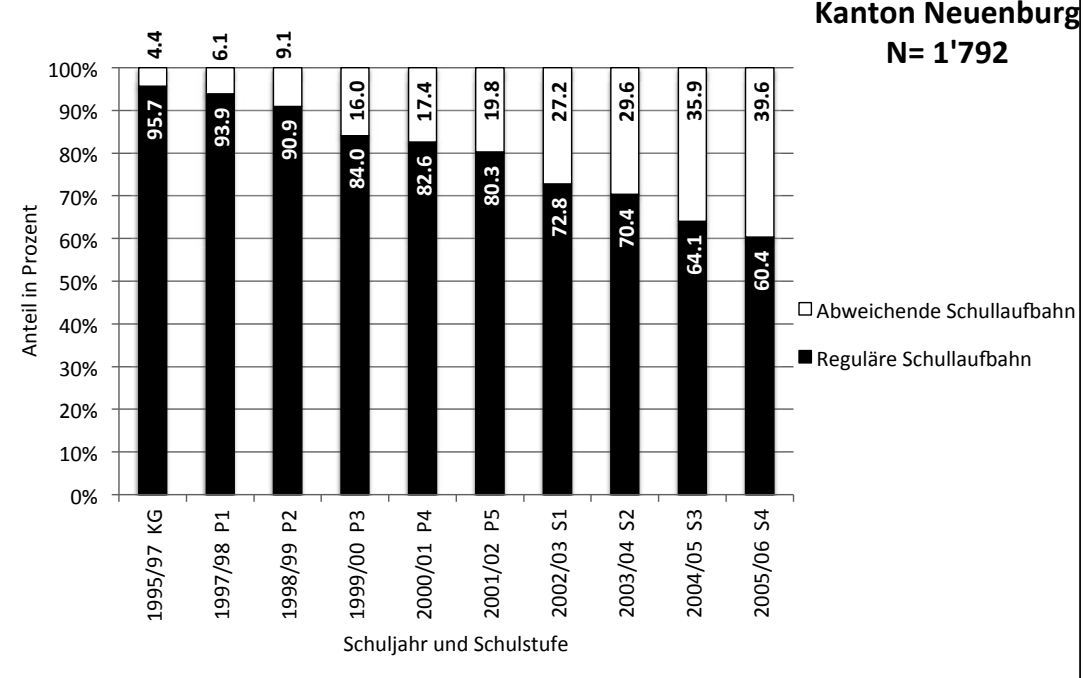

Abb. 6 Schullaufbahnen im Kanton Neuenburg der Kohorte 1994/96 bis 2005/06 (Vonlanthen, 2011)

Abbildung 7 zeigt die Schullaufbahnen der Aargauer Kohorte für die Schuljahre 2000/01 bis 2008/09. Die Analyse bezieht sich hier auf die Primar- und Sekundarstufe I, ohne Kindergarten. Aus der Aargauer Kohorte gelingt es auch nur jedem zweiten Kind, die obligatorische Schule (Primar- und Sekundarstufe I) regulär zu durchlaufen. Von den 5‘544 Kindern besuchten 4‘738 den Kindegarten. 15.0\% davon hatten bereits im Kindergarten eine abweichende Laufbahn. Hochgerechnet ist davon auszugehen, dass in dieser Kohorte lediglich etwa $40 \%$ der Schülerinnen und Schüler die obligatorische Schule ohne Abweichung durchlaufen haben. Damit übertrifft der Kanton Aargau die Kantone Neuenburg und Freiburg deutlich. Trotz gewisser Unterschiede müssen alle drei Kantone als hoch selektiv bezeichnet werden. Auch wenn die beschriebenen drei Kohorten teilweise schon älteren Datums sind, muss darauf hingewiesen werden, dass die Ergebnisse über die Zeit sehr stabil sind. Inwiefern die Umsetzungen von HarmoS und des Konkordates „Sonderpädagogik“ an der Situation etwas verändern, müssen erneute Analysen zeigen. 


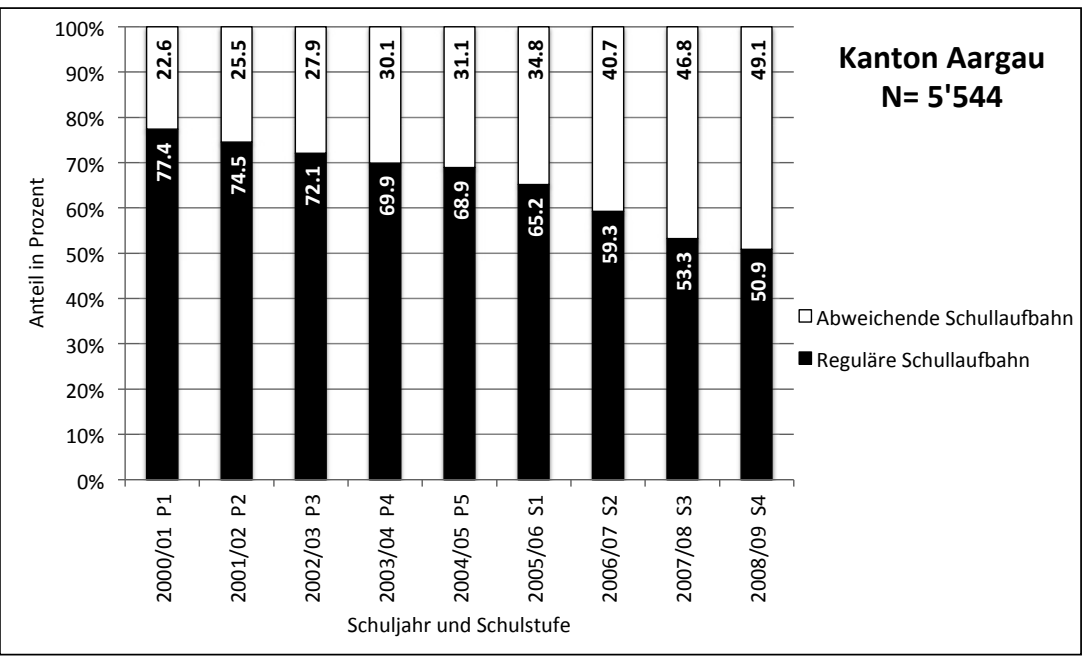

Abb. 7 Schullaufbahnen, ohne Kindergarten, im Kanton Aargau der Kohorte 2000/01 bis $2008 / 09$ (Lüthi, 2014)

Die Frage, welche Formen die abweichenden Schullaufbahnen aufweisen, liegt nahe. Vorliegende Befunde sind in Tabelle 3 dokumentiert. Dabei muss beachtet werden, dass die Angaben nur die erste Schullaufbahnabweichung berücksichtigen. Zahlreiche Kinder erfahren im Laufe ihrer Schulzeit mehrere Selektionsmassnahmen.

In den Kantonen Freiburg und Neuenburg steht vor allem die Klassenwiederholung im Vordergrund (was generell für die Kantone der Romandie zutrifft). Etwa $30 \%$ der Schülerinnen und Schüler wiederholen mindestens eine Klasse. Berücksichtigt man den Umstand, dass negative und positive Typenwechsel auf der Sekundarstufe I mehrheitlich ebenfalls Klassenwiederholungen sind, so ist davon auszugehen, dass mehr als ein Drittel der Schülerschaft mindestens ein Mal eine Klasse wiederholt. Der Kanton Aargau ist diesbezüglich etwas zurückhaltender, wobei anstelle der Klassenwiederholung andere Massnahmen deutlich häufiger ergriffen werden, zum Beispiel die Aussonderung (13.7\%), der Typenwechsel auf der Sekundarstufe I (12.2\%) oder die verspätete Einschulung (9.0\%, in der Rubrik „andere“ aufgenommen). 
Tab. 3 Merkmale abweichender Schullaufbahnen in den Kantonen Freiburg, Neuenburg und Aargau (vgl. Taminelli, 2007; Vonlanthen, 2011; Lüthi, 2014)

\begin{tabular}{lrrc}
\hline Schullaufbahnen & \multicolumn{1}{c}{ FR } & \multicolumn{1}{c}{ NE } & \multicolumn{1}{c}{$\mathrm{AG}^{*}$} \\
\hline Aussonderung & $3.7 \%$ & $1.7 \%$ & $13.7 \%$ \\
Klassenwiederholung & $30.1 \%$ & $29.2 \%$ & $13.2 \%$ \\
Typenwechsel auf Sek I & $8.7 \%$ & $3.7 \%$ & $12.2 \%$ \\
andere & $4.7 \%$ & $5.0 \%$ & $10.0 \%$ \\
\hline Reguläre Schullaufbahn & $52.8 \%$ & $60.4 \%$ & $50.9 \%$ \\
\hline
\end{tabular}

* ohne Kindergarten, womit die Vergleichbarkeit nicht vollständig gegeben ist.

In Anbetracht dieser Zahlen stellt sich unweigerlich die Frage nach der Wirksamkeit der verordneten Massnahmen zum Wohle des Kindes. Die empirische Befundlage der Integrationsforschung attestiert der Aussonderung, insbesondere von Kindern mit erheblichen Lernschwierigkeiten, eine geringe Effizienz. Zur Wirksamkeit der Typenwechsel auf der Sekundarstufe I sowie zur verspäteten Einschulung liegen für die Schweiz keine systematischen Untersuchungen vor. Wie es um die Wirksamkeit der Klassenwiederholung steht, ist Gegenstand des nächsten Abschnitts.

\section{$3 \quad$ Forschungsergebnisse zur Klassenwiederholung}

Im nun folgenden knappen Forschungsüberblick werden einerseits die Wirksamkeit der Klassenwiederholung auf die Entwicklung der Schulleistungen und andererseits der Einfluss der Klassenwiederholung auf die weitere Laufbahn dargestellt.

\subsection{Wirkung der Klassenwiederholung auf die Lernentwicklung}

Frühere Studien, welche vorwiegend aus den USA stammen, zeigen insgesamt negative Effekte der Klassenwiederholung auf die Entwicklung der Schulleistungen. Repetierende schneiden in den darauffolgenden Jahren schlechter ab als regulär promovierte Peers, auch wenn die relevanten Leistungsvariablen und familiale Variablen kontrolliert werden. Folgende Forschungsüberblicke und Metaanalysen stützen diese Aussage: Bless, Schüpbach \& Bonvin, 2004; Dewaine Brigth, 2011; Jackson, 1975; Holmes \& Matthews, 1984; Holmes, 1989; Smith \& Shepard, 1987; Hess, 2010; und Martin, 2011. 
Untersuchungen ab 1990 sind in der Regel Längsschnittstudien mit einer sorgfältigen Kontrolle von Störvariablen, mit Kontrollgruppen und mit unterschiedlichen Vergleichen der Leistungsentwicklung: Same-age-Vergleich und/oder Same-grade-Vergleich (Abbildung 8). Methodisch betrachtet gelten die neueren Untersuchungen als zuverlässig.

Bei den Same-age-Vergleichen zeigt sich eine signifikant schlechtere Lernentwicklung der Repetierenden im Vergleich zu Schülerinnen und Schülern, welche in die nächst höhere Klasse promoviert wurden (Allen, Chen, Wilson \& Hughes, 2009; Bless et al., 2004; Holmes, 1989; Hong \& Raudenbush, 2005; Jimerson, 2001; Jimerson, 2004; McCoy \& Reynolds, 1999; Silberglitt, Appleton, Burns \& Jimerson, 2006a; Wu, West \& Hughes, 2008a).

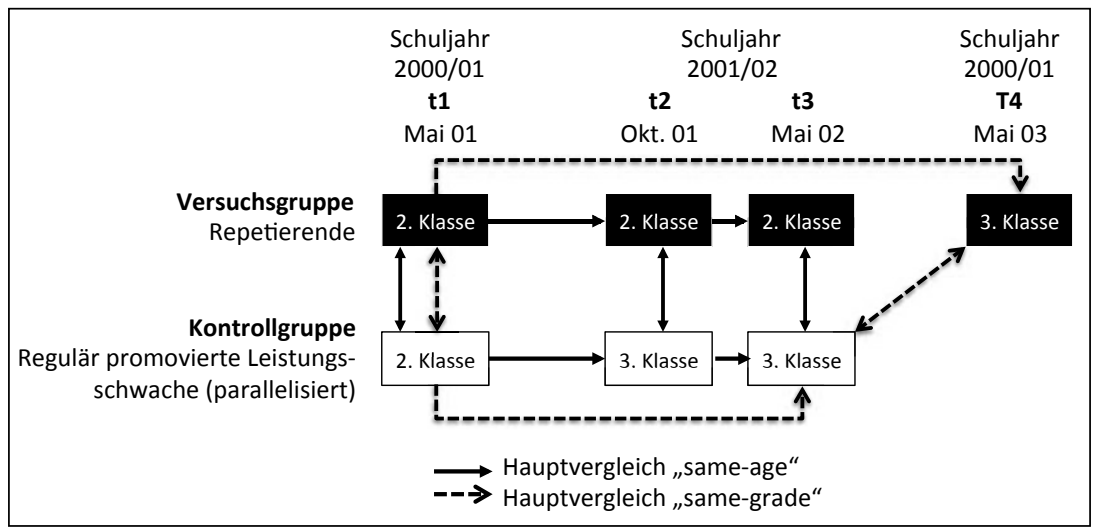

Abb. 8 Beispiel eines Forschungsdesigns mit Same-age- und Same-grade-Vergleich (Bless et al., 2004, 58)

Bei Same-grade-Vergleichen ergibt sich zunächst eine positivere Lernentwicklung der Repetierenden gegenüber vergleichbaren Schülerinnen und Schülern, welche in die nächste Klasse promoviert wurden. Allerdings ist dieser Leistungsvorteil nur im ersten Jahr (Repetitionsjahr) festzustellen. Anschliessend fallen die Repetierenden deutlich zurück, und nach spätestens drei Schuljahren sind keine Effekte der Klassenwiederholung mehr nachweisbar (Alexander, Entwisle \& Dauber, 1994; Allen et al., 2009; Bless et al., 2004; Holmes, 1989; Karweit, 1999; Moser, West \& Hughes, 2012; Pierson \& Connell, 1992; Roderick \& Nagoka, 2005; Ou \& Reynolds, 2010; Silberglitt et al., 2006a; Wu et al., 2008a). 
Gemäss der aktuellen Forschungslage scheint der Zeitpunkt der Klassenwiederholung keine Rolle hinsichtlich ihrer Effizienz zu spielen (Alexander et al., 1994; Ou \& Reynolds, 2010; Silberglitt et al., 2006b; Wu et al., 2008b; vgl. auch den Forschungsüberblick von Hess, 2010). Somit ist die Wirksamkeit der Klassenwiederholung im Hinblick auf mittel- bis längerfristige Fortschritte in den Schulleistungen nicht gegeben. Unter Berücksichtigung der Forschungslage ist die aufgezeigte Häufigkeit, mit der Klassenwiederholungen angeordnet werden, kritisch zu hinterfragen.

\subsection{Klassenwiederholung und die weitere Laufbahn}

Besorgniserregend ist die Langzeitwirkung der Klassenwiederholung. Die Repetition einer Klasse wird zu einer wichtigen Prädiktorvariablen für einen späteren negativen Verlauf der Schulkarriere, welche durch eine hohe Dropout-Rate, durch eine erhöhte Selektion in Schultypen mit geringerem Anspruchsniveau und durch Sonderschulung gekennzeichnet ist (Alexander et al., 1994; Alexander, Entwisle, Dauber \& Kabbani, 2004; Hennemann, Hagen \& Hillenbrand, 2010; Fine \& Davis, 2003; Jimerson, 2001; Hess, 2010; Ou \& Reynolds, 2010).

Zum Einfluss der Klassenwiederholung auf die berufliche Ausbildung, auf die Bildung nach der Sekundarstufe II, auf die Arbeitssituation, den sozioökonomischen Status sowie auf die Etablierung in der Gesellschaft liegen nur einzelne Ergebnisse vor, sodass es zurzeit nicht möglich ist, verlässliche Aussagen zu treffen. Es scheint jedoch so zu sein, dass die Klassenwiederholung auch in den genannten Bereichen als Prädiktorvariable mit negativem Einfluss in Frage kommt (Fine \& Davis, 2003; Jimerson, 1999; Keller \& Moser, 2013; Ou \& Reynolds, 2010).

\section{$4 \quad$ Schlussfolgerungen}

Die bildungsstatistischen Analysen zeigen die Selektivität der Schweizer Bildungssysteme. In einigen Kantonen gelingt es nahezu jedem 2. Schulkind nicht, die obligatorische Schule regulär zu durchlaufen. Das ist gesellschaftspolitisch und ökonomisch relevant. Bildungsstatistische Analysen auf der Basis von Individualdaten sind im Vergleich zu Analysen auf der Basis von Daten pro Schuljahr besser geeignet, um das Ausmass der getroffenen Selektionsmassnahmen zu analysieren und die Schullaufbahnen umfassend zu beschreiben. Analysen von Daten pro Schuljahr haben die Tendenz, die Problematik zu verharmlosen. Mit dem Forschungsüber- 
blick zur Wirksamkeit der Klassenwiederholung konnte im Sinne eines Beispiels gezeigt werden, dass diese Selektionsmassnahme, welche zum Wohle des Kindes ergriffen wird, im Widerspruch zu ihrer häufigen Anwendung, nicht halten kann, was man sich von ihr verspricht. Es ist zu vermuten, dass bei anderen Selektionsmassnahmen dieselbe Problematik besteht. Mit jeder Selektionsentscheidung geht das Bildungssystem das Risiko ein, sowohl Fehlentscheidungen zu treffen, die sich bei weiteren Selektionsentscheidungen kumulieren können, als auch Massnahmen $\mathrm{zu}$ ergreifen, welche sich nicht als genügend wirksam erweisen.

Im Sinne von Forschungsperspektiven für die Zukunft wurde versucht, Analysen von Schullaufbahnen mittels Individualdaten gesamtschweizerisch und pro Kanton anzuregen. Zudem ist die Forschung künftig gefordert, die Wirksamkeit der Selektionsmassnahmen vermehrt zu untersuchen, und zwar sowohl in Bezug auf ihre Haupt- als auch in Bezug auf ihre Nebenwirkungen.

\section{Literatur}

Alexander, K. L., Entwisle, D. R., \& Dauber, S. L. (1994). On the Success of a Failure: A reassessment of the effects of retention in the primary grades. Cambridge: University Press.

Alexander, K. L., Entwistle, D. R., Dauber, S. L., \& Kabbani, N. (2004). Dropout in relation to grade retention: An accounting from the beginning school study. In A. J. Walberg, A. J. Reynolds, \& M. C. Wanf (Eds.), Can unlike students learn together? Grade retention, traching, and grouping (pp. 5-34). Greenwich: Information Age Publishing.

Allen, C. S., Chen, Q., Willson, V. L., \& Hughes, J. N. (2009). Qualitiy of research design moderates effects of grade retention on achievemnt: a meta-analytic, multi-level analysis. Educational evaluation and policy analysis, 31 (4), 480-499. doi: 10.3102/0162373709352239.

Bless, G., Schüpbach, M., \& Bonvin, P. (2004). Klassenwiederholung - Determinanten, Wirkungen und Konsequenzen. Bern Stuttgart Wien: Haupt.

Bundesamt für Statistik (2017, 27. Februar). http://www.bfs.admin.ch/bfs/portal/de/index/ themen/15.html.

Dewayne Bright, A. (2011). A meta-analysis oft he effects of grade retention of $K-6$ students on student achievement. 1990-2010. Dissertation submitted in the Department of Educational Leadership, Policy, and Technology Studies in the Graduate School oft he University of Alabama.

Donzé, R. (2015, 25. Oktober). Förderung schwacher Schüler - Die Klasse wiederholen war einmal. Neue Zürcher Zeitung NZZ. Zugriff unter http://www.nzz.ch/nzzas/nzz-amsonntag/die-klasse-wiederholen-war-einmal

Fend, H. (2006). Neue Theorie der Schule. Einführung in das Verstehen von Bildungssystemen. Wiesbaden: VS Verlag für Sozialwissenschaften.

Fine, J. G., \& Davis, J. M. (2003). Grade retention and enrollment in post-secondary education. Journal of School Psychology, 41, 401-411. 
Gomolla, M., \& Radtke, F. O. (2009). Institutionelle Diskriminierung. Die Herstellung ethnischer Differenz in der Schule. (3. Auflage) Wiesbaden: VS Verlag für Sozialwissenschaften.

Hennemann, T.; Hagen, T. \& Hillenbrand, C. (2010). Dropout aus der Schule - Empirisch abgesicherte Risikofaktoren und wirksame pädagogische Massnahmen. Empirische Sonderpädagogik, 3, 26-47.

Hess, R. (2010). Retention. In Clauss-Ehlers, C. S. (Ed.), Enccyclopedia of Cross-Cultural School Psychology (Band 2, 818-821). New York: Springer.

Holmes, C. T. (1989). Grade-level retention effects: A meta-analysis of research studies. In L. A. Shepard, \& M. L. Smith (Eds.), Flunking grades: Research and policies on retention (pp. 16-33). London: Falmer Press.

Holmes, C. T., \& Matthews, K. M. (1984). The effects of nonpromotion on elementary and junior high school pupils: A meta-analysis. Review of Educational Research, 54(2), 225-236.

Hong, G., \& Raudenbush, S. W. (2005). Effects of kindergarten retention policy on childrens's cognitive growth in reading and mathematics. Educational Evaluation and Policy Analysis , 27(3), 205-224.

Jackson, G. B. (1975). The research evidence on the effects of grade retention. Review of Educational Research, 45(4), 613-635.

Jimerson, S. R. (1999). On the failure of failure: Examining the association between early grade retention and education and employment outcomes during late adolescence. Journal of School Psychology, 37(3), 243-272.

Jimerson, S. R. (2001). Meta-analysis of grade retention research: Implications for practice in the 21st century. School Psychology Review, 30(3), 420-437.

Jimerson, S. R. (2004). Is grade retention educational malpractice? Empirical evidence from meta-analyses examining the efficacy of grade retention. In H. J. Walberg, A. J. Reynolds, \& M. C. Wang (Eds.), Can unlike students learn together? Grade retention, tracking, and grouping (pp. 71-96). Greenwich, CT: Information Age Publishing.

Karweit, N.L. (1999). Grade retention: Prevalence, timing, effects. (Report 33). Baltimore: John Hopkins University, CRESPAR.

Keller, F. \& Moser, U. (2013). Schullaufbahnen und Bildungserfolg, Aiswirkungen von Schullaufbahnen und Schulsystem auf den Übertritt ins Berfufsleben. Zürich-Chur: Rüegger Verlag.

Lüthi, A. (2014). Schullaufbahnen im Kanton Aargau - Eine populationsbeschreibende Untersuchung zum Verlauf der obligatorischen Schulzeit an Aargauer Schulen. Masterarbeit eingereicht bei der Philosophischen Fakultät der Universität Freiburg $(\mathrm{CH})$.

Martin, A. J. (2011a). Holding back and holding behind: grade retention and students' non-academic and academic outcomes. British Educational Research Journal, 37(5), 739-763.

Martin, A. J. (2011b). Grade retention: Effects on Academic and Non-academic Development. In R. J. Levesque (Ed.), Ecyclopedia of Adolescence (Band 2, pp. 1207-1213). New York: Springer.

McCoy, A. R., \& Reynolds, A. J. (1999). Grade retention and school performance: An extended investigation. Journal of School Psychology, 37(3), 273-298.

Moser, S. E., West, S. G., \& Hughes, J. N. (2012). Trajectories of math and reading achievement in low-achieving children in elementary school: effects of early and later retention in grade. Journal of Education Psychology, 104(3), 603-621.

Ou, S. R., \& Reynolds, A. J. (2010). Grade retention, post-secondary education and public aid receipt. Educational Evaluation and Policy Analysis, 32, 118-139. 
Pierson, L. H., \& Connell, J. P. (1992). Effects of grade retention on self-system processes, school engagement, and academic performance. Journal of Educational Psychology, 84(3), 300-307.

Roderick, M., \& Nagaoka, J. (2005). Retention under Chicago's high-stakes testing program: Helpful, harmful, or harmless? Educational Evaluation and Policy Analysis, 27(4), 309-340.

Silberglitt, B., Appleton, J. J., Burns, M. K., \& Jimerson, S. R. (2006a). Examining the effects of grade retention on student reading performance: A longitudinal study. Journal of School Psychology, 44, 255-270.

Silberglitt, B., Jimerson, S. R., Burns, M. R., \& Appleton, J. J. (2006b). Does the timing of grade retention make a difference? Examining the effects of early versus late retention. School Psychology Review, 35(1), 134-141.

Smith, M. L., \& Shepard, L. A. (1987). What doesn't work: Explaining policies of retention in the early grades. Phi Delta Kappan, 66, 129-134.

Taminelli, Ch. (2007). Parcours scolaire régulier ou irrégulier? Description du parcours scolaire des élèves fribourgeois qui ont débuté leur scolarité en 1992/1993. Mémoire de Licence présenté à la Faculté des Lettres de l'Université de Fribourg $(\mathrm{CH})$.

Vonlanthen, M. (2011). Trajectoires scolaires - Etude d'une volée d'élèves neuchâtelois. Mémoire de Master présenté à la Faculté des Lettres de l'Université de Fribourg $(\mathrm{CH})$.

Wu, W., West, S. G., \& Hughes, J. N. (2008a). Short-term effects of grade retention on the growth rate of Woodcock-Johnson III broad math and reading scores. Journal of School Psychology, 46, 85-105.

Wu, W., West, S. G., \& Hughes, J. N. (2008b). Effect of retention in first grade on children's achievement trajectories over 4 years: A piecewise growth analysis using propensity score matching. Journal of Educational Psychology, 100(4), 727-740.

Open Access Dieses Kapitel wird unter der Creative Commons Namensnennung 4.0 International Lizenz (http://creativecommons.org/licenses/by/4.0/deed.de) veröffentlicht, welche die Nutzung, Vervielfältigung, Bearbeitung, Verbreitung und Wiedergabe in jeglichem Medium und Format erlaubt, sofern Sie den/die ursprünglichen Autor(en) und die Quelle ordnungsgemäß nennen, einen Link zur Creative Commons Lizenz beifügen und angeben, ob Änderungen vorgenommen wurden.

Die in diesem Kapitel enthaltenen Bilder und sonstiges Drittmaterial unterliegen ebenfalls der genannten Creative Commons Lizenz, sofern sich aus der Abbildungslegende nichts anderes ergibt. Sofern das betreffende Material nicht unter der genannten Creative Commons Lizenz steht und die betreffende Handlung nicht nach gesetzlichen Vorschriften erlaubt ist, ist für die oben aufgeführten Weiterverwendungen des Materials die Einwilligung des jeweiligen Rechteinhabers einzuholen.

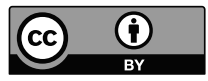




\section{Soziale Ungleichheiten im Leistungszuwachs und bei Bildungsülbergängen}

Urs Moser, Jeannette Oostlander und Martin J. Tomasik

\section{Zusammenfassung}

Dieser Beitrag beschreibt den längsschnittlichen Lernzuwachs in den Fächern Mathematik und Deutsch während der obligatorischen Schulzeit. Die Grundlage dafür bildet eine Zufallsstichprobe von etwa 2000 Schülerinnen und Schülern aus dem Kanton Zürich, die im Sommer 2003 eingeschult worden sind. Neben der Beschreibung der Schulleistungen wird überprüft, in welchem Ausmass sich diese durch die soziale Herkunft und die Erstsprache vorhersagen lässt. Ausserdem wird untersucht, inwieweit soziale Herkunft und Erstsprache die Wahrscheinlichkeit eines Übertritts in ein Langgymnasium über die Schulleistungen hinaus erklären können. Es zeigt sich, dass die Schulleistungen auf der Primarstufe stark und auf der Sekundarstufe nur noch mässig ansteigen. Die Abflachung des Lernzuwachses lässt sich inbesondere im Fach Mathematik beobachten. Während die soziale Herkunft sehr stark mit den Schulleistungen zusammenhängt, zeigt sich, dass die Erstsprache nach Kontrolle der sozialen Herkunft keinen eigenständigen Effekt mehr hat. Die soziale Herkunft hat schliesslich einen grossen Einfluss auf den Übertritt in ein Langgymnasium, und zwar selbst nach Kontrolle der Schulleistungen und der übertrittsrelevanten Noten. Es wird geschlussfolgert, dass allfällige Programme zum Nachteilausgleich der sozialen Herkunft früh ansetzen müssen, um die sozialen Disparitäten in den Lernvoraussetzungen zu reduzieren.

\section{Schlagworte}

Lernzuwachs, Schullaufbahn, Längsschnittstudie, soziale Ungleichheiten, Bildungsübergänge 


\section{$1 \quad$ Lernzuwachs während der Schulzeit}

In der empirischen Bildungsforschung geht es häufig darum, die Ergebnisse von Bildungssystemen quantitativ messbar zu machen und Faktoren für den Bildungserfolg zu identifizieren. In diesem Kapitel sollen beide Aspekte aufgegriffen werden. Dabei möchten wir uns bei den Ergebnissen vor allem auf die Wirkungen des Bildungssystems im Kanton Zürich konzentrieren, wie sie sich in Schulleistungen und deren Zuwachs über die obligatorische Schulzeit hinweg widerspiegeln. Dieser Zuwachs wird üblicherweise in Schuljahresäquivalenten angegeben, wobei die Veränderungen der Leistungen pro Schuljahr mit ihrer Streuung in Relation gesetzt werden, um somit ein relatives Mass für Veränderungen zu erhalten, das über verschiedene Klassenstufen und Schultypen vergleichbar ist.

In Querschnittstudien, die unterschiedliche Klassenstufen miteinander verglichen haben, zeigen sich vergleichbare Schuljahresäquivalente im Bereich von etwa einem Drittel einer Standardabweichung. In TIMSS-II beispielsweise zeigte sich ein durchschnittlicher Zuwachs zwischen der Klassenstufe 7 und 8 von $d=.29$, wobei die Effektstärken zwischen Ländern (Schweiz: $d=.40$; Deutschland: $d=.25$; Baumert \& Lehmann, 1997) und Schultypen (Gymnasium in Deutschland: $d=.31$; Hauptschule in Deutschland $d=.16$; Lehmann, Gänsfuß \& Peek, 1999) erheblich variierten. Wößmann (2005) berichtet auf Grundlage der PISA-2000-Daten einen Leistungszuwachs zwischen den Klassenstufen 9 und 10 von $d=.30$ für Mathematik, $d=.32$ für Naturwissenschaften und $d=.33$ für Lesen.

Auch auf niedrigeren Klassenstufen findet sich ein Lernzuwachs ähnlich hoher Grössenordnung. So berichten Lehmann und Seeber (2004) auf der Grundlage der LAU-Längsschnittstudie eine Effektstärke von $d=.34$ pro Schuljahr vom Anfang der Klassenstufe 5 bis zum Ende der Klassenstufe 6 im Fach Mathematik. Für höhere Klassenstufen finden die Autoren eine Abflachung des Lernzuwachses. Zwischen dem Beginn der Klassenstufe 7 und dem Ende der Klassenstufe 8 verändert sich die Leistung in Mathematik nur noch um $d=.27$, mit starken Unterschieden zwischen den Schultypen (Gymnasium in Deutschland: $d=.24$; Hauptschule in Deutschland: $d=.50)$. Zwischen dem Anfang der Klassenstufe 9 und dem Ende der Klassenstufe 11 sank dann der Lernzuwachs auf $d=.21$ (Gymnasium in Deutschland), was nunmehr etwa einem Fünftel einer Standardabweichung entspricht. Hinweise für ein ähnliches Abflachen des Lernzuwachses finden sich auch bei Bloom, Hill, Black und Lipsey (2008). 


\section{Erstsprache und soziale Herkunft als Prädiktoren des Lernzuwachses}

Die oben skizzierten Zuwächse stellen Durchschnittswerte für einzelne Klassenstufen oder Schultypen dar. Innerhalb dieser Verläufe gibt es allerdings sehr viel interindividuelle Varianz, die sich durch Variablen wie Geschlecht oder schulisches Selbstkonzept erklären lässt. Im Mittelpunkt dieses Kapitels stehen die Erstsprache und die soziale Herkunft als Prädiktoren des Lernzuwachses im Vordergrund. Diese beiden Faktoren zeigen in vielen Studien relativ hohe Effektstärken und lassen sich damit als wichtige individuelle Determinanten des Kompetenzerwerbs begreifen. Gleichzeitig haben sie auch eine soziale Komponente; denn starke Effekte dieser Variablen widersprechen dem bildungspolitischen Ziel von Chancengleichheit (vgl. Geißler, 2004).

\subsection{Erstsprache}

Das Beherrschen der Unterrichtssprache stellt eine der wichtigsten Lernvoraussetzungen dar, um sich am Unterricht beteiligen zu können und um letztlich vom Unterricht zu profitieren (Zöller, Roos \& Schöler, 2006). Das gilt nicht nur für Schulfächer, welche Sprache direkt zum Gegenstand haben, sondern auch und vielleicht insbesondere für den mathematisch-naturwissenschaftlichen Bereich (Prediger \& Özdil, 2011).

Als theoretische Erklärung für den Nachteil von Kindern aus Migrationsfamilien wird häufig die These der Entwicklungsinterdependenz von Cummins (1991) ins Spiel gebracht. Hierbei geht man davon aus, dass sprachliche und kognitive Entwicklung eng zusammenhängen, so dass die kognitive Entwicklung gefährdet ist, wenn die bilingualen Schülerinnen und Schüler es nicht schaffen, sowohl in der Herkunfts- als auch in der Zweitsprache einen ausreichend elaborierten Sprachstand zu erreichen. Obwohl die These von Cummins aus linguistischer Perspektive oft kritisiert worden ist (z. B. Tracy, 2005), lässt sich nicht verneinen, dass das Erlernen einer Zweitsprache zunächst kognitive Ressourcen kostet und zusätzliche Lernzeit erfordert, die an anderer Stelle fehlt (Hopf, 2005).

Eine weitere, ergänzende Erklärung für eine nachteilige schulische Entwicklung als Folge von Migration und der damit häufig verbundenen Zweisprachigkeit gibt die Humankapitalhypothese. Sie beruht auf der Beobachtung, dass Migration häufig mit einem niedrigeren sozioökonomischen Status konfundiert ist (z. B. Ramm, Prenzel, Heidemeier \& Walter, 2005), der ursächlich für die nachteilige Entwicklung der Schulleistungen ist. Für diese Hypothese würde sprechen, dass 
der Unterschied in den Leistungen zwischen einheimischen und zugewanderten Kindern in den Ländern, die eine restriktive und hochselektive Einwanderungspolitik ausüben, relativ niedrig ausfällt (Ehmke, Siegle \& Hohensee, 2005). Diese Beobachtung führt uns zur Frage nach der Rolle der sozialen Herkunft für den Bildungserfolg und den damit verbundenen Mechanismen.

\subsection{Soziale Herkunft}

Bildungsbeteiligung und Kompetenzerwerb hängen stark mit der sozialen Herkunft der Schülerinnen und Schüler zusammen, wie unlängst grosse internationale Vergleichsstudien gezeigt haben (z. B. Baumert \& Schümer, 2001). Die Schweiz bildet hier keine Ausnahme. Vielmehr ist der Zusammenhang zwischen Leistung und sozialer Herkunft sogar höher als in den meisten anderen Ländern, und zwar auch unter Einbezug der kognitiven Grundfähigkeiten (Ramseier \& Brühwiler, 2003).

Soziale Ungleichheiten können sich durch mindestens vier Prozesse reproduzieren, wobei die empirische Befundlage zu den einzelnen Prozessen jeweils mehr oder weniger gut gesichert ist (vgl. Maaz, Baumert \& Trautwein, 2009). Erstens zeigt sich ein sozial selektives Entscheidungsverhalten von Eltern und Jugendlichen an Bildungsübergängen in Verbindung mit entsprechendem Beratungs- und Empfehlungsverhalten von Erzieherinnen und Lehrkräften. Dass dieses selektive Entscheidungs- und Empfehlungsverhalten von primären und sekundären Sozialschichteffekten gleichermassen beeinflusst ist, ist mittlerweile empirisch gut belegt (z. B. Paulus \& Blossfeld, 2007).

Zweitens können innerhalb von Bildungsinstitutionen Disparitäten als Folge einer Wechselwirkung zwischen Statusmerkmalen und der Nutzung von schulischen Lerngelegenheiten entstehen, wobei häufig Habitustheorien (Bourdieu, 1973) oder selektive Erwartung- und Wertschätzungsstrukturen (Helsper, Kramer, Hummrich \& Busse, 2009) zur Erklärung herangezogen werden. Der empirische Nachweis dieser Prozesse ist allerdings nicht besonders überzeugend.

Drittens kann eine frühe institutionelle Ausdifferenzierung von Bildungsprogrammen Ungleichheiten beim Kompetenzerwerb verursachen. Viele empirische Befunde zeigen, dass die unterschiedlichen Lern- und Entwicklungsmilieus, wie sie etwa durch ein mehrgliedriges Schulsystem gegeben sind, zu heterogenen Leistungsergebnissen führen (vgl. Maaz, Trautwein, Lüdtke \& Baumert, 2008).

Schliesslich spielen ausserhalb von Bildungsinstitutionen auch die Entwicklungskontexte Nachbarschaft oder Freizeit eine Rolle. Häufig werden diese Effekte in sogenannten "Sommerlochstudien“ untersucht, in denen die Leistungsentwicklung in der Ferienzeit beobachtet und damit der direkte Einfluss von Bildungsinstitu- 
tionen minimiert wird. Empirische Hinweise für steigende soziale Disparitäten zeigen sowohl Studien aus den USA (z. B. Alexander, Entwisle \& Olson, 2001) als auch aus Europa (z. B. Lindahl, 2001).

Unabhängig von den zugrunde liegenden Mechanismen gibt es empirische Belege dafür, dass sich die sozialen Gradienten zwischen den Schulsystemen in den einzelnen Ländern unterscheiden (Ehmke \& Baumert, 2008). Auch zwischen regional kleineren Einheiten mit ähnlicheren Bildungssystemen zeigen sich zuweilen grosse Disparitäten, wie der Blick auf die Schweizer Kantone zeigt (Moser \& Berweger, 2005).

\section{$3 \quad$ Fragestellungen}

In diesem Kapitel stellen wir uns zunächst die Frage, ob sich der durchschnittliche Leistungszuwachs in Höhe von etwa einem Drittel Standardabweichung pro Schuljahr auch in der Zürcher Stichprobe replizieren lässt. Dazu werden die Schulleistungen in den Fächern Deutsch und Mathematik betrachtet. Im Gegensatz zu den vielen querschnittlichen Studien soll die Frage hier im Längsschnitt (3., 6. und 9. Unterrichtsjahr) beantwortet werden. Zusätzlich interessierte uns, ob sich das in einigen Studien gefundene Abflachen des Lernzuwachses vor allem im Fach Mathematik auch in unseren Daten beobachten lässt.

Im zweiten Schritt möchten wir uns anschauen, welche Rolle die Erstsprache und die soziale Herkunft für den Verlauf der Leistungen in den beiden Fächern spielen. Insbesondere wollten wir wissen, ob Lernende mit Deutsch als Zweitsprache sowie solche mit benachteiligter sozialer Herkunft niedrigere Ausgangsleistungen und/oder Lernzuwächse zeigen. Ausserdem wollten wir wissen, ob diese Disparitäten auch dann bestehen, wenn man die kognitive Leistungsfähigkeit statistisch kontrolliert.

Abschliessend wollen wir uns fragen, ob Erstsprache und soziale Herkunft über die Schulleistungen und deren Bewertung hinaus die Wahrscheinlichkeit des Übertritts in ein Langgymnasium nach der 6. Klasse beeinflussen. Sollte das der Fall sein, wäre das ein Hinweis auf das oben erwähnte selektive Entscheidungsverhalten an Bildungsübergängen. 


\section{$4 \quad$ Methode}

\subsection{Stichprobe und Datenerhebung}

Die Grundgesamtheit der Längsschnittstichprobe der Zürcher Lernstanderhebung bildeten alle Lernenden, die im Sommer 2003 im Kanton Zürich in eine 1. Klasse eingetreten sind. Die Stichprobenziehung erfolgte klassenweise, wobei explizit nach Schultyp (Regelklassen, Regelklassen in Reformschulen und Kleinklassen) und implizit nach dem Sozialindex und der Klassengrösse stratifiziert wurde. Aus den ausgewählten Klassen wurden sämtliche 2046 Schülerinnen und Schüler in die Studie aufgenommen und zur Teilnahme eingeladen. Davon nahmen effektiv $N_{1}=1970$ an der Erhebung zum Anfang des Schuljahres 2003/04 teil (vgl. Moser \& Stamm, 2005).

Tab. 1 Bildungssituation am Ende der obligatorischen Schulzeit

\begin{tabular}{lccccr}
\hline & 7. Klasse & 8. Klasse & 9. Klasse & 10. Klasse & Total \\
\hline Gymnasium & & $0,7 \%$ & $17,3 \%$ & $0,5 \%$ & $18,5 \%$ \\
Abteilung A & & $4,5 \%$ & $32,8 \%$ & & $37,2 \%$ \\
Abteilung B & $0,1 \%$ & $7,9 \%$ & $24,3 \%$ & & $32,3 \%$ \\
Abteilung C & $0,1 \%$ & $1,9 \%$ & $2,3 \%$ & & $4,2 \%$ \\
Kleinklasse/Sonderschule & & $1,2 \%$ & & $0,2 \%$ & $1,3 \%$ \\
Privatschule & $0,1 \%$ & $1,6 \%$ & $3,9 \%$ & & $5,5 \%$ \\
Berufslehre & & & & $0.3 \%$ & $0,3 \%$ \\
Handels-/Fachmittelschule & & & $0,6 \%$ & $0,1 \%$ & $0,7 \%$ \\
\hline Total & $0,2 \%$ & $17,6 \%$ & $81,1 \%$ & $1,1 \%$ & $100 \%$ \\
\hline
\end{tabular}

Am Ende des Schuljahres 2005/06 reduzierte sich die Stichprobe vor allem durch Wegzug von Schülerinnen und Schülern aus dem Kanton auf nunmehr 1960 Kinder, die alle zur Teilnahme an der zweiten Erhebung eingeladen wurden. Von diesen nahmen am Ende $N_{2}=1935$ effektiv teil (vgl. Keller \& Moser, 2008a). Am Ende des Schuljahres 2008/09 hatte sich die Stichprobe durch Wegzug von weiteren 157 Kindern auf 1803 Schülerinnen und Schüler reduziert, von denen insgesamt $N_{3}=$ 1679 an der dritten Erhebung teilnahmen (vgl. Angelone \& Moser, 2011). Die vierte Erhebung fand Ende des Schuljahres 2011/12 statt und basierte auf einer Stichprobe von 1817 Lernenden. Die Verteilung der Jugendlichen auf die unterschiedlichen Schultypen und Klassenstufen findet sich in Tabelle 1, woraus ersichtlich wird, dass sich eine grosse Mehrheit zu diesem Messzeitpunkt in der 9. und damit letzten 
obligatorischen Klassenstufe befand. Weitere 17,9\% wiederholten eine Klassenstufe und $1,1 \%$ waren bereits in der 10. Klasse. An dieser Erhebung nahmen insgesamt $N_{4}=1635$ Jugendliche teil. Weitere Details zu diesem Messzeitpunkt finden sich bei Angelone, Keller und Moser (2013, Kap. 2.1).

\subsection{Erhebungsinstrumente}

Für die Bestimmung des Lernstands auf den Klassenstufen 3, 6 und 9 wurden auf Grundlage des Lehrplans und der Lehrmittel des Kantons Zürich jeweils ein Deutsch- und ein Mathematiktest durch qualifizierte Lehrpersonen entwickelt und durch das Institut für Bildungsevaluation erprobt. Eine Auswahl von Aufgabenund Lösungsbeispielen für die 3. Klasse findet sich bei Keller und Moser (2008a, 2008b), für die 6. Klasse bei Moser und Angelone (2011b) und für die 9. Klasse bei Angelone et al. (2013, Kap. 2.2.2).

Die Variable Erstsprache unterscheidet zwischen Kindern mit Deutsch als Erst- und solchen mit Deutsch als Zweitsprache. Die Erstsprache wurde erfasst als Sprache, welche die Eltern am häufigsten mit ihrem Kind sprechen. Die Angaben stammen aus den Elternfragebögen, die zu verschiedenen Testzeitpunkten eingesetzt wurden. Zwei Drittel der Kinder sprachen Deutsch als Erstsprache und ein Drittel als Zweitsprache.

Die allgemeinen kognitiven Fähigkeiten wurden zum ersten Erhebungszeitpunkt beim Schuleintritt mit dem Culture Fair Test (CFT 1; Weiss \& Osterland, 1997) erfasst. Bezüglich der kriteriumsbezogenen Validität zeigen sich mittlere Korrelationen zwischen der CFT 1-Gesamtleistung und dem HAWIK (Handlungsteil $r=$ .66 und Verbalteil $r=.48$; Weiss \& Osterland, 1997).

Zur Bestimmung der sozialen Herkunft der Schülerinnen und Schüler wurde ein Index der verschiedenen Ressourcen des Elternhauses gebildet. Als Indikator für die ökonomischen Ressourcen wurde die Anzahl der Zimmer der Familienwohnung pro Personen berücksichtigt. Die bildungsrelevanten Ressourcen wurden anhand der Anzahl der Bücher im Haushalt und dem höchsten Bildungsabschluss der Eltern erfasst. Aus diesen drei Angaben wurde mit Hilfe der Hauptkomponentenanalyse ein Index gebildet. Die Streubreite in der Stichprobe reicht von -2.77 bis 3.51 Indexpunkten bei $M=.00(S D=1.00)$. Für einen Teil der weiteren Analysen wurden die Schülerinnen und Schüler nach der sozialen Herkunft in vier gleich grosse Gruppen eingeteilt. 


\subsection{Stichprobenselektivität}

Während der Stichprobenausfall pro Erhebung zum grössten Teil auf Schulabsenzen und Teilnahmeverweigerung zurückzuführen ist, liegt der Stichprobenausfall bezogen auf die Ausgangsstichproben zum grössten Teil an der geografischen Mobilität über die Kantonsgrenzen hinweg. Um zu überprüfen, wie sehr sich die Zusammensetzung der Längsschnittstichprobe durch den Stichprobenausfall verändert, wurden die einzelnen Messzeitpunkte auf den untersuchungsrelevanten Merkmalen Geschlecht, Erstsprache, kognitive Grundfähigkeit und soziale Herkunft verglichen. Die Ergebnisse legen nahe, dass die Zusammensetzung der effektiven Stichprobe zu allen Messzeitpunkten nahezu identisch ist.

\section{$5 \quad$ Skalierung der Leistungstests}

Die Ergebnisse der Schülerinnen und Schüler in allen Leistungstests wurden nach dem Rasch-Modell (de Ayala, 2009) skaliert. Die Grundannahme des Rasch-Modells ist, dass die Lösungswahrscheinlichkeit einer Aufgabe von zwei Merkmalen abhängt, nämlich von der Fähigkeit des Schülers oder der Schülerin und von der Aufgabenschwierigkeit. Der grosse Vorteil der Rasch-Skalierung ist, dass beide Merkmale auf ein und derselben, metrischen Skala abgebildet werden können. Damit kann umgekehrt die Fähigkeit des Schülers oder der Schülerin aus der Lösungswahrscheinlichkeit einer Aufgabe und ihrer Schwierigkeit bestimmt werden. Details zur verwendeten Rasch-Skalierung und den damit zusammenhängenden Skalenanalysen finden sich bei Keller und Moser (2008a) sowie bei Angelone et al. (2013, Kap. 2.4).

Die Rasch-Skalierung erleichtert nicht nur eine inhaltliche Interpretation der Testergebnisse, indem man für jede Schülerin und jeden Schüler Rückschlüsse aus der Fähigkeit auf die Lösungswahrscheinlichkeit jeder Aufgabe ziehen kann, für die der Schwierigkeitsparameter bekannt ist. Ein weiterer, in unserem Zusammenhang zentraler Vorteil ist es auch, dass sich die Fähigkeiten von Schülerinnen und Schülern direkt vergleichen lassen, die unterschiedliche und unterschiedlich schwierige Items gelöst haben (vgl. Kolen \& Brennan, 2004). Das erlaubt den direkten Vergleich der Fähigkeiten über mehrere Schuljahre hinweg auf ein und derselben metrischen Skala, obwohl sich die Leistungen zwischen diesen Schuljahren zuweilen erheblich unterscheiden. Voraussetzung für diesen Vergleich ist allerdings das Vorhandensein sogenannter Link-Aufgaben, deren Schwierigkeit sich mit der Schwierigkeit der eigentlich interessierenden Aufgaben überlappt (vgl. de Ayala, 2009, Kap. 11). 
Das Prinzip dieses sogenannten Common-item-nonequivalent-groups-design, das von uns Verwendung gefunden hat, ist in Abbildung 1 dargestellt. Um beispielsweise die Leistungen der Schülerinnen und Schüler der 3. Klasse mit denen der 6. Klasse zu vergleichen, wurden in der 3. Klasse Aufgaben zur Lösung vorgelegt, die auch in einer Zusatzstichprobe von Schülerinnen und Schülern der 4. Klasse gelöst wurden. Diese wiederum fanden zum Teil Verwendung in einer Zusatzstichprobe aus der 5. Klasse usw. Durch die Bestimmung der Schwierigkeitsparameter jeder einzelnen Aufgabe auf jeder einzelnen Klassenstufe auf einer gemeinsamen metrischen Skala liessen sich die Aufgaben zwischen weiter entfernten Klassenstufen miteinander vergleichen.

\begin{tabular}{|c|c|c|c|c|}
\hline Klasse & & Link 3-4 & Link 4-5 & Link 5-6 \\
\hline 3. & Test 3. Klasse & Test 4. Klasse & & \\
\hline 4. & & Test 4. Klasse & Test 5. Klasse & \\
\hline 5. & & & Test 5. Klasse & Test 6. Klasse \\
\hline 6. & & & & Test 6. Klasse \\
\hline
\end{tabular}

Abb. 1 Das verwendete Common-item-nonequivalent-groups-design

\section{$6 \quad$ Ergebnisse}

\subsection{Leistungszuwachs in Deutsch und Mathematik}

Zur Berechnung des Leistungszuwachses in Deutsch und Mathematik wurden separat für beide Fachbereiche quadratische Zwei-Ebenen-Wachstumsmodelle (random intercept- und random slope-Modelle) mit drei Messzeitpunkten gerechnet. Dabei waren die abhängigen Variablen die Deutsch- und Mathematikleistungen nach drei, sechs und neun Schuljahren. Die unabhängigen Variablen auf Ebene 1 waren die Anzahl der Schuljahre sowie der quadratische Term der Anzahl der Schuljahre. Die unabhängigen Variablen auf Ebene 2 bestanden aus dem fachspezifischen Vorwissen beim Schuleintritt, den kognitiven Grundfähigkeiten, der sozialen Herkunft, der Erstsprache und dem Geschlecht des Schülers oder der Schülerin. Diese werden im Folgenden als individuelle Lernvoraussetzungen bezeichnet. Die Effektstärken $d$ werden jeweils als Schuljahresäquivalente angegeben. 
In Deutsch stiegen die Leistungen um durchschnittlich $d=.44$ zwischen dem 3. und dem 6. Unterrichtsjahr und um $d=.31$ zwischen dem 6. und dem 9. Unterrichtsjahr. Damit liegt der Leistungszuwachs mit im Durchschnitt $d=.38$ etwas über einem Drittel einer Standardabweichung. Es deutet sich aber eine Verlangsamung des Leistungszuwachses zum Ende der obligatorischen Schulzeit an. Die Leistungskurve ist im linken Teil der Abbildung 2 dargestellt.

In Mathematik steigen die Leistungen zwischen dem 3. und dem 6. Unterrichtsjahr um $d=.78$ und zwischen dem 6 . und dem 9. Unterrichtsjahr um $d=.14$. Insgesamt liegt der Leistungszuwachs mit $d=.45$ pro Jahr deutlich über einem Drittel einer Standardabweichung, was aber vor allem an dem starken Leistungszuwachs in den ersten drei untersuchten Unterrichtsjahren liegt. In den letzten drei Unterrichtsjahren zeigt sich dagegen eine deutliche Verlangsamung des Leistungszuwachses. Die Leistungskurve für Mathematik ist im rechten Teil der Abbildung 2 dargestellt.

\subsection{Leistungszuwachs in Deutsch und Mathematik nach Erstsprache}

Abbildung 3 zeigt den Verlauf des Leistungszuwachses im Fach Deutsch in Abhängigkeit von der Erstsprache. Es fällt auf, dass sich die Verläufe kaum im Anstieg (Deutsch als Erstsprache: $d=.40$; Deutsch als Zweitsprache: $d=.39$ ) unterscheiden, wohl aber sehr deutlich im Niveau. Im 6 . Unterrichtsjahr beispielsweise beträgt der Unterschied 67 Leistungspunkte $(d=.67)$. Diese Unterschiede verschwinden fast vollständig, wenn man für die individuellen Lernvoraussetzungen statistisch kontrolliert.

Ein ähnliches Bild zeigt sich für den Leistungszuwachs im Fach Mathematik, der in Abbildung 4 dargestellt ist. Die Verläufe unterscheiden sich kaum in der Form oder in der Steigung (Deutsch als Erstsprache: $d=.48$; Deutsch als Zweitsprache: $d=.48$ ), aber sehr deutlich im Niveau. So beträgt der Leistungsunterschied im 6 . Schuljahr 53 Leistungspunkte $(d=.54)$. Wie im Fach Deutsch verschwindet dieser Unterschied nahezu, wenn man die individuellen Lernvoraussetzungen kontrolliert. 


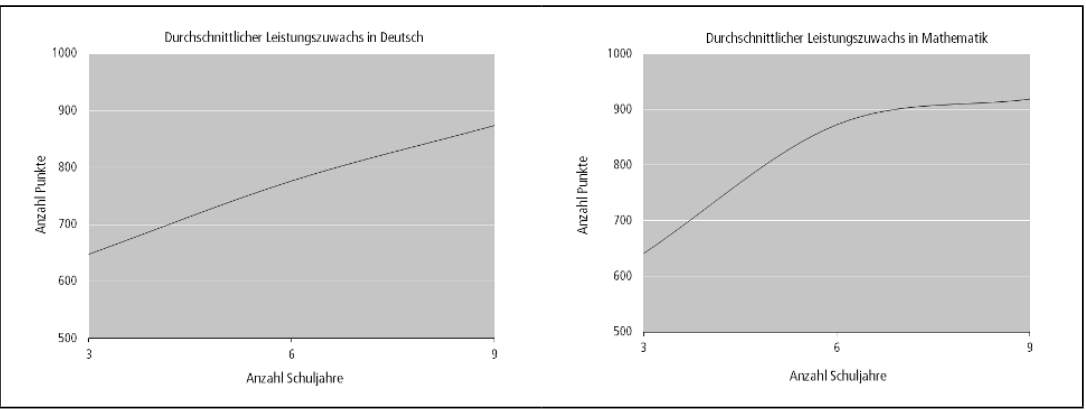

Abb. 2 Leistungszuwachs in Deutsch und Mathematik (Quelle: Bildungsdirektion Kanton Zürich, 2014)

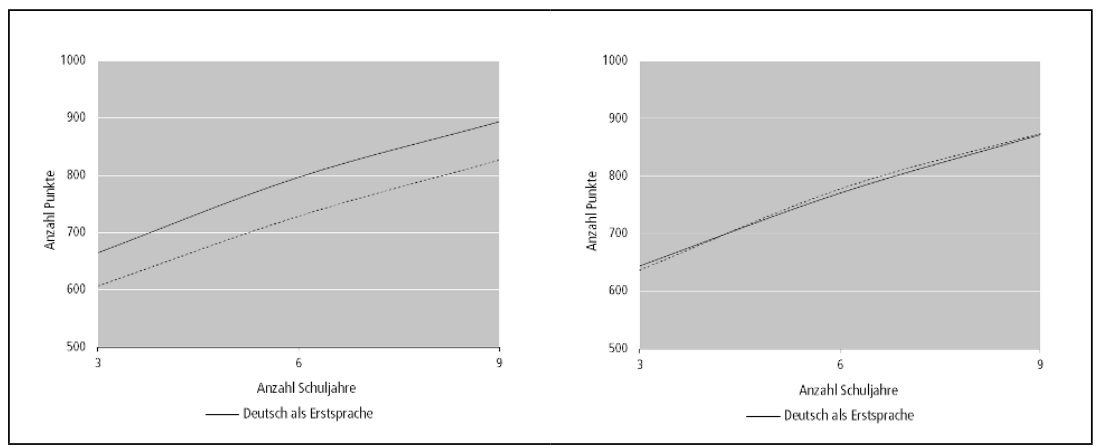

Abb. 3 Leistungszuwachs in Deutsch nach Erstsprache ohne und mit statistischer Kontrolle der Lernvoraussetzungen (Quelle: Bildungsdirektion Kanton Zürich, 2014)

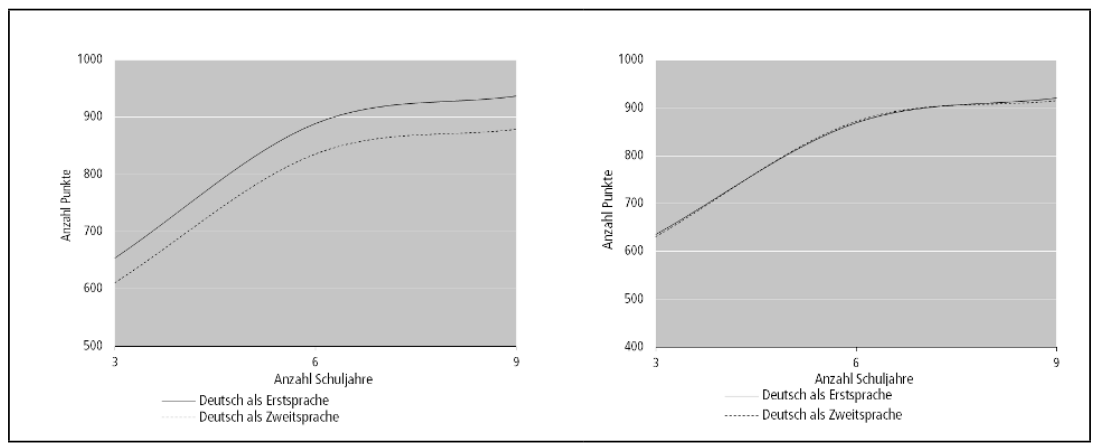

Abb. 4 Leistungszuwachs in Deutsch nach sozialer Herkunft ohne und mit statistischer Kontrolle der Lernvoraussetzungen (Quelle: Bildungsdirektion Kanton Zürich, 2014) 

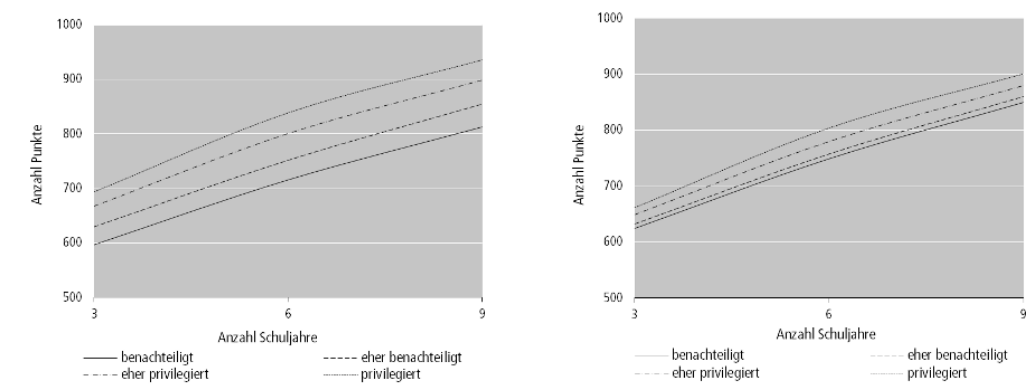

Abb. 5 Leistungszuwachs in Mathematik nach Erstsprache ohne und mit statistischer Kontrolle der Lernvoraussetzungen (Quelle: Bildungsdirektion Kanton Zürich, 2014)

\subsection{Leistungszuwachs in Deutsch und Mathematik nach sozialer Herkunft}

Bezüglich sozialer Herkunft zeigt sich, dass sich der Anstieg der Lernleistungen in Deutsch und Mathematik weniger zwischen den einzelnen Gruppen unterscheidet, wohl aber im Ausgangsniveau. Im Fach Deutsch zeigt sich der grösste Unterschied im Anstieg zwischen der benachteiligten (1. Quartil; $d=.40$ ) und der privilegierten Gruppe (4. Quartil; $d=.44)$. Dies ist vor allem auf Anstiege zwischen der 3. und 6. Klasse (1. Quartil: $d=.44$; 4. Quartil: $d=.53$ ) und nahezu gar nicht auf Unterschiede zwischen der 6. und der 9. Klasse (1. Quartil: $d=.35 ; 4$. Quartil $d=.34$ ) zurückzuführen. Diese beiden Gruppen unterscheiden sich auch erwartungsgemäss in ihrem Niveau am stärksten. In der 6. Klasse beträgt der Unterschied 123 Leistungspunkte $(d=1.31)$. In Mathematik findet sich der grösste Unterschied im Anstieg zwischen der eher benachteiligten (2. Quartil; $d=.48$ ) und der privilegierten Gruppe (4.Quartil; $d=.50$ ). Im Niveau der 6. Klasse unterscheidet sich die benachteiligte von der privilegierten Gruppe mit 114 Leistungspunkten $(d=1.19)$ am stärksten. Wie aus Abbildung 5 deutlich wird, verschwinden die Unterschiede im Niveau selbst nach statistischer Kontrolle der Lernvoraussetzungen nicht vollständig, sondern bleiben erhalten. 


\subsection{Wahrscheinlichkeit des Übertritts in das Lang- gymnasium nach Erstsprache und sozialer Herkunft}

Um zu beurteilen, welche Rolle die Erstsprache und die soziale Herkunft für die Wahl des Schultyps nach dem Ende der Primarschule spielt, haben wir die Übertrittswahrscheinlichkeiten in einer multiplen logistischen Regression als Funktion der Erstsprache und der sozialen Herkunft modelliert. Als zusätzliche erklärende Variablen wurden das Geschlecht der Schülerin oder des Schülers, das Alter sowie die Zeugnisnoten und die Leistungen in Deutsch und Mathematik aufgenommen. Zusätzlich wurde die Teilnahme an einer privaten Prüfungsvorbereitung auf das Langgymnasium statistisch kontrolliert. Die Ergebnisse finden sich in Tabelle 2, wobei an dieser Stelle nur auf das vollständige Modell 3 eingegangen werden soll.

Deutlich wird, dass sowohl die Zeugnisnoten als auch die Leistungen einen wichtigen Beitrag zur Erklärung der Übertrittswahrscheinlichkeit leisten, und zwar jeweils einen eigenständigen. So sagen bessere Noten eine höhere Übertrittswahrscheinlichkeit vorher, wenn man Leistungen statistisch kontrolliert, ebenso wie bessere Leistungen eine höhere Übertrittswahrscheinlichkeit vorhersagen, wenn man Noten statistisch kontrolliert. Deutsch als Zweitsprache spielt dagegen keine Rolle, wenn alle anderen Variablen berücksichtigt werden. Dagegen ist die soziale Herkunft selbst nach Kontrolle aller anderen Variablen hochgradig prädiktiv für einen Übertritt.

Tab. 2 Ergebnisse der binär logistischen Regression zur Vorhersage des Übertritts in das Langgymnasium nach der 6. Klasse

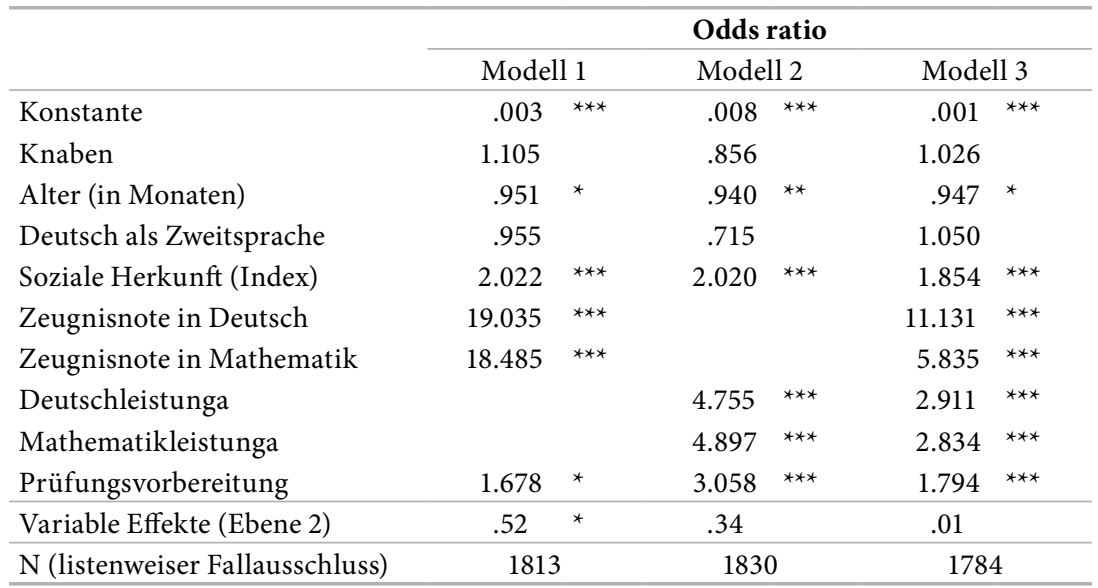

Anmerkungen: ${ }^{a}$ Koeffizient bezogen auf je 100 Leistungspunkte; ${ }^{*} p<.05 ;{ }^{* *} p<.01 ;{ }^{* * *} p<.001$ 
Dieser Effekt ist in Abbildung 6 beispielhaft dargestellt. In ihr wird die Übertrittswahrscheinlichkeit, welche durch die soziale Herkunft moderiert wird, als Funktion der Zeugnisnoten dargestellt. Als Beispiel ist die Note eingezeichnet, bei der die Übertrittswahrscheinlichkeit in der Gesamtstichprobe $50 \%$ beträgt (etwa 5.5 oder zwischen "gut" und „sehr gut"). Mit dieser Note haben Schülerinnen und Schüler mit privilegierter sozialer Herkunft - selbst wenn alle oben genannten Variablen statistisch kontrolliert werden - eine $71 \%$ ige Wahrscheinlichkeit, nach der 6 . Klasse in das Langgymnasium zu wechseln, während diese Wahrscheinlichkeit bei benachteiligter sozialer Herkunft bei nur $29 \%$ liegt.

\section{Diskussion}

Im vorliegenden Kapitel haben wir anhand der längsschnittlichen Daten der Zürcher Lernstanderhebung bestimmt, wie sich die Leistungen während der obligatorischen Schulzeit entwickeln und welche Rolle die Erstsprache und die soziale Herkunft der Schülerinnen und Schüler für die Kompetenzentwicklung während dieser Zeit sowie für den Bildungsweg spielen. Als erster Befund bleibt festzuhalten, dass sich neun Jahre nach der Einschulung vier von fünf der Lernenden in der 9. Klassenstufe befanden und knapp jeder Fünfte mindestens eine Klassenstufe wiederholt hatte. Eine Klassenstufenwiederholung war dabei relativ häufig auf den weniger anspruchsvollen Abteilungen der nicht-gymnasialen Sekundarschulen anzutreffen.

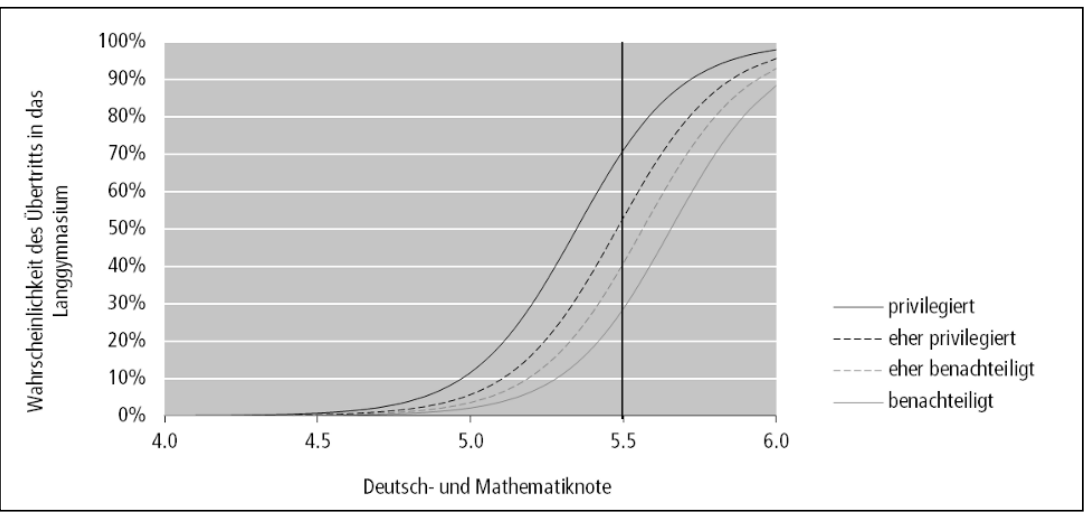

Abb. 6 Wahrscheinlichkeit des Übertritts in das Langgymnasium (Quelle: Bildungsdirektion Kanton Zürich, 2011) 
Die Leistungsentwicklung der Zürcher Schülerinnen und Schüler lag insgesamt etwas über dem in der Literatur häufig berichteten einen Drittel einer Standardabweichung pro Schuljahr. Der Leistungszuwachs war dabei in Mathematik etwas grösser als in Deutsch, verlief aber keineswegs linear über die gesamte Schulzeit hinweg. Es zeigte sich eine Verlangsamung des Zuwachses, wobei es zwischen den beiden Schulfächern und Schulstufen grosse Unterschiede gab. Während auf der Primarstufe der Zuwachs in Mathematik deutlich grösser war als in Deutsch, zeigte sich auf der Sekundarstufe I eine starke Verlangsamung vor allem im Fach Mathematik. Die Grössenordnung dieser Verlangsamung ist ein unerwarteter Befund und lässt sich im Nachhinein vielleicht am besten mit Unterschieden in der Fachdidaktik erklären. Während auf der Primarstufe neue mathematische Sachverhalte erarbeitet und immer wieder gefestigt werden, zeichnet sich der Mathematikunterricht auf der Sekundarstufe I stärker durch jahrgangsspezifische Lerninhalte aus, die seltener wieder aufgegriffen und durch Üben konsolidiert werden. Natürlich bedarf diese Interpretation weiterer empirischer Überprüfung, wie sie beispielsweise durch den systematischen Vergleich unterschiedlicher fachdidaktischer Konzepte erfolgen könnte.

Bezüglich der Erstsprache hat sich gezeigt, dass diese zwar mit dem Ausgangsniveau korreliert war, jedoch kaum einen Einfluss auf den Leistungszuwachs während der obligatorischen Schulzeit hatte. Das gilt insbesondere, wenn die soziale Herkunft der Schüler statistisch kontrolliert wurde. Schülerinnen und Schüler mit Deutsch als Zweitsprache scheinen also über die obligatorische Schulzeit hinweg vom Unterricht genauso gut zu profitieren zu haben wie jene, die die Unterrichtssprache als Erstsprache sprachen. Erhärtet wird dieser Befund auch dadurch, dass die Erstsprache keinen eigenständigen Erklärungswert für die Übertrittswahrscheinlichkeit in das Langgymnasium hatte. Insgesamt scheint dieser Befund für die Humankapitalhypothese zu sprechen, die von einer Konfundierung der beiden Variablen Erstsprache und soziale Herkunft ausgeht und der letzteren eine kausale Rolle in der Vorhersage von Schulleistungen und Bildungslaufbahnen zuspricht. Gleichzeitig spricht sie gegen Erklärungsversuche, welche im Bilingualismus an sich einen Risikofaktor für Schulerfolg sehen.

Im Gegensatz zur Erstsprache hatte die soziale Herkunft der Schülerinnen und Schüler einen stärkeren Einfluss auf die Leistungsentwicklung und insbesondere auf die Übertrittswahrscheinlichkeit in das Langgymnasium. Abgesehen davon, dass Schülerinnen und Schüler mit niedrigerer sozialer Herkunft auf einem niedrigerem Ausgangsniveau starteten, zeigte sich bei der Leistungsentwicklung in Deutsch (nicht aber in Mathematik) ein Schereneffekt im Bereich der Primarschule, der zu grösseren Disparitäten und nicht etwa zu der erhofften Homogenisierung der Leistungen führte. Besonders deutlich wurde der Effekt der sozialen Herkunft bei der Übertrittswahrscheinlichkeit auf das Langgymnasium und dort insbesondere 
in einem "mittleren“ Leistungsbereich, wo die Entscheidung für oder gegen das Langgymnasium besonders ambivalent ist. Dieser Befund unterstützt die von Maaz et al. (2009) postulierte zentrale Rolle von Bildungsübergängen als Erklärung für die Reproduktion sozialer Ungleichheit. Ob weitere Mechanismen eine Rolle spielen, kann an dieser Stelle nicht geklärt werden.

\subsection{Schlussfolgerungen für Forschung und Praxis}

Aus den vorliegenden Befunden ergeben sich verschiedene Schlussfolgerungen für Forschung und Praxis, wobei an dieser Stelle lediglich auf drei eingegangen werden soll. Zum einem wird aus den Ausführungen deutlich, welchen Informationsvorteil langfristige, längsschnittliche Untersuchungen gegenüber Studien haben, die Leistungsunterschiede zwischen Klassenstufen querschnittlich untersuchen. Es braucht unseres Erachtens eine breitere längsschnittliche Datenbasis zum Vergleich verschiedener Bildungssysteme, die sich idealerweise in den schulischen Curricula oder im Timing der Bildungsübergänge unterscheiden, um Alterseffekte von Unterrichtseffekten zu trennen. Auch der Vergleich aufeinanderfolgender Kohorten erscheint wünschenswert, um den Einfluss von Perioden- und Kohorteneffekten identifizieren zu können.

Zum anderen braucht es angesichts der Konfundierung von Sprach-, Migrationsund sozialen Herkunftseffekten intelligente Studiendesigns, die es erlauben, diese Effekte nicht nur statistisch sondern faktisch voneinander zu trennen. Der Vergleich von Ländern mit unterschiedlicher Migrationspolitik wurde in der Einleitung als Beispiel angeführt, aber solche Designs leiden natürlich am Problem, dass sich durch die Auflösung einer Konfundierung wiederum andere Konfundierungen, etwa durch unterschiedliche Bildungssysteme, ergeben. Im besten Fall würde man experimentelle Designs entwickeln und die Effekte individueller oder bildungspolitischer Interventionen gegen eine randomisierte Kontrollgruppe testen. Solche Studien sind aber naturgemäss teuer, dauern lange und könnten zuweilen auch aus einer forschungsethischen Perspektive problematisch sein.

Schneller und einfacher umzusetzen, sind vermutlich Interventionen, die sich an die tägliche Lehrpraxis oder Schulsozialarbeit richten (z. B. Schellenberg \& Häfeli, 2009). Angesichts des starken Effekts der sozialen Herkunft auf die Bildungsverläufe scheint es mehr als angebracht, solche Programme zu fördern und zu evaluieren. Die hier vorgestellten Befunde legen nahe, dass solche Programme möglichst früh ansetzen sollten, um soziale Disparitäten in den Lernvoraussetzungen nach Möglichkeit zu reduzieren, damit diese nicht über die gesamte Bildungslaufbahn hinweg weitergetragen werden. 


\section{Literaturverzeichnis}

Alexander, K. L., Entwisle, D. R., \& Olson, L. S. (2001). Schools, achievement, and inequality: A seasonal perspective. Educational Evaluation and Policy Analysis, 23, 171-191.

Angelone, D., Keller, F., \& Moser, U. (2013). Entwicklung schulischer Leistungen während der obligatorischen Schulzeit: Bericht zur vierten Zürcher Lernstandserhebung zuhanden der Bildungsdirektion des Kantons Zürich. Zürich: Bildungsdirektion.

Angelone, D., \& Moser, U. (2011). Die Zürcher Lernstandserhebung im Überblick. In Bildungsdirektion des Kantons Zürich (Hrsg.), Nach sechs Jahren Primarschule: Deutsch, Mathematik und motivational-emotionales Befinden am Ende der 6. Klasse (S. 119-128). Zürich: Bildungsdirektion.

Baumert, J., \& Lehmann, R. (Hrsg.). (1997). TIMSS - Mathematisch-naturwissenschaftlicher Unterricht im internationalen Vergleich: Deskriptive Befunde. Opladen: Leske + Budrich.

Baumert, J., \& Schümer, G. (2001). Familiäre Lebensverhältnisse, Bildungsbeteiligung und Kompetenzerwerb. In Deutsches PISA-Konsortium (Hrsg,), PISA 2000 - Basiskompetenzen von Schülerinnen und Schülern im internationalen Vergleich (S.323-407). Opladen: Leske + Budrich.

Bildungsdirektion Kanton Zürich (2011). Nach sechs Jahren Primarschule: Lernstand der Schulanfängerinnen und Schulanfänger von 2003 vor ihrem Übertritt in die Sekundarstufe $I$ [Broschüre]. Zürich: Bildungsdirektion.

Bildungsdirektion Kanton Zürich (2014). Nach neun Jahren Schule: Entwicklung der schulischen Leistungen von Schülerinnen und Schülern im Kanton Zürich während der obligatorischen Schulzeit [Broschüre]. Zürich: Bildungsdirektion.

Bloom, H. S., Hill, C. J., Black, A. R., \& Lipsey, M. W. (2008). Performance trajectories and performance gaps as achievement effect-size benchmarks for educational interventions. Journal of Research on Educational Effectiveness, 1, 289-328.

Bourdieu, P. (1973). Kulturelle Reproduktion und soziale Reproduktion. In P. Bourdieu, Grundlagen einer Theorie der symbolischen Gewalt (S. 88-137). Frankfurt a. M.: Suhrkamp.

Cummins, J. (1991). Interdependecy in first and second language proficiency in bilingual children. In E. Bialystock (Ed.), Language processing in bilingual children (pp. 70-89). Cambridge, UK: Cambridge University Press.

de Ayala, R. J. (2009). The theory and practice of item response theory. New York: Guilford. Ehmke, T., \& Baumert, J. (2008). Soziale Disparitäten des Kompetenzerwerbs und der Bildungsbeteiligung in den Ländern: Vergleiche zwischen PISA 2000 und 2006. In M. Prenzel, C. Artelt, J. Baumert, W. Blum, W. Hammann, E. Klieme, \& R. Pekrum (Hrsg.), PISA 2006 in Deutschland: Die Kompetenzen der Jugendlichen im dritten Ländervergleich (S.319-342). Münster: Waxmann.

Ehmke, T., Siegele, T., \& Hohensee, F. (2005). Soziale Herkunft im Ländervergleich. In M. Prenzel, J. Baumert, W. Blum, R. Lehmann, D. Leutner, M. Neubrand, R. Pekrun, J. Rost \& U. Schiefele (Hrsg.), PISA 2003: Der zweite Vergleich der Länder in Deutschland - Was wissen und können Jugendliche? (S. 235-268). Münster: Waxmann.

Geißler, R. (2004). Die Illusion der Chancengleichheit im Bildungssystem - von PISA gestört. Zeitschrift für Soziologie der Erziehung und Sozialisation, 24, 362-380.

Grensch, C. (2012). Der Übergang in die Sekundarstufe I: Leistungsbeurteilung, Bildungsaspiration und rechtlicher Kontext bei Kindern mit Migrationshintergrund. Wiesbaden: VS Verlag für Sozialwissenschaften. 
Helpser, W., Kramer, R.-T., Hummrich, M., \& Busse, S. (2009). Jugend zwischen Familie und Schule: Eine Studie zu pädagogischen Generationenbeziehungen. Wiesbaden: VS Verlag für Sozialwissenschaften.

Hopf, D. (2005). Zweisprachigkeit und Schulleistung bei Migrantenkindern. Zeitschrift für Pädagogik, 51, 236-251.

Keller, F., \& Moser, U. (2008a). Die Untersuchung im Überblick. In U. Moser \& J. Hollenweger (Hrsg.), Drei Jahre danach: Lesen, Wortschatz, Mathematik und soziale Kompetenzen am Ende der dritten Klasse (S.13-40). Oberentfelden: Sauerländer.

Keller, F., \& Moser, U. (2008b). Fachleistungen am Ende der 3. Klasse. In U. Moser \& J. Hollenweger (Hrsg.), Drei Jahre danach: Lesen, Wortschatz, Mathematik und soziale Kompetenzen am Ende der dritten Klasse (S. 41-87). Oberentfelden: Sauerländer.

Kolen, M. J., \& Brennan, R. L. (2004). Test equating, scaling, and linking: Methods and practices. New York: Springer.

Lehmann, R. H., Gänsfuß, R., \& Peek, R. (1999). Aspekte der Lernausgangslage und Lernentwicklung von Schülerinnen und Schülern an Hamburger Schulen - Klassenstufe 7: Bericht über die Untersuchung im September 1998. Hamburg: Behörde für Schule und Berufsbildung.

Lehmann, R. H., \& Seeber, S. (2004). „Accelerated mathematics“ in grades 4 through 6: Evaluation of a quasi-experimental study in 15 schools of North Rhine-Westphalia, Germany. Wisconsin Rapids, WI: Renaissance Learning.

Lindahl, M. (2001). Summer learning and the effect of schooling: Evidence from Sweden (IZA Discussion Paper No. 262). Bonn: IZA.

Maaz, K., Baumert, J., \& Trautwein, U. (2009). Genese sozialer Ungleichheit im institutionellem Kontext der Schule: Wo entsteht und vergrössert sich soziale Ungleichheit? In J. Baumert, K. Maaz \& U. Trautwein (Hrsg.), Bildungsentscheidungen (Zeitschrift für Erziehungswissenschaft, Sonderheft 12, S. 11-46). Wiesbaden: VS Verlag für Sozialwissenschaften.

Maaz, K., Trautwein, U., Lüdtke, O., \& Baumert, J. (2008). Educational transitions and differential learning environments: How explicit between-school tracking contributes to social inequality in educational outcomes. Child Development Perspectives, 2, 99-106.

Moser, U., \& Angelone, D. (2011). Fachleistungen am Ende der 6. Klasse. In Bildungsdirektion des Kantons Zürich (Hrsg.), Nach sechs Jahren Primarschule: Deutsch, Mathematik und motivational-emotionales Befinden am Ende der 6. Klasse (S. 31-49). Zürich: Bildungsdirektion Kanton Zürich.

Moser, U., \& Berweger, S. (2005). Soziale Herkunft und Mathematikkompetenz: Ein vertiefter Blich auf die Kantone. In Bundesamt für Statistik (BFS) \& Schweizerische Konferenz der kantonalen Erziehungsdirektoren (EDK) (Hrsg.), PISA 2003: Kompetenzen für die Zukunft - Zweiter nationaler Bericht (S.99-118). Neuchâtel/Bern: BFS/EDK.

Moser, U., \& Stamm, M. (2005). Die Untersuchung im Überblick. In U. Moser, M. Stamm \& J. Hollenweger (Hrsg.), Für die Schule bereit? Lesen, Wortschatz, Mathematik und soziale Kompetenzen beim Schuleintritt (S. 13-26). Oberentfelden: Sauerländer.

Paulus, W., \& Blossfeld, H.-P. (2007). Schichtspezifische Präferenzen oder sozioökonomisches Entscheidungskalkül: Zur Rolle elterlicher Bildungsaspirationen im Entscheidungsprozess beim Übergang von der Grundschule in die Sekundarstufe. Zeitschrift für Pädagogik, 53, 491-508.

Prediger, S., \& Özdil, E. (Hrsg.). (2011). Mathematiklernen unter Bedingungen der Mehrsprachigkeit: Stand und Perspektiven der Forschung und Entwicklung in Deutschland (Mehrsprachigkeit Bd. 32). Münster: Waxmann. 
Ramm, G., Prenzel, M., Heidemeier, H., \& Walter, O. (2004). Soziokulturelle Herkunft: Migration. In PISA-Konsortium Deutschland (Hrsg.), PISA 2003: Der Bildungsstand der Jugendlichen in Deutschland - Ergebnisse des zweiten internationalen Vergleichs (S. 254-272). Münster: Waxmann.

Ramseier, E., \& Brühwiler, C. (2003). Herkunft, Leistung und Bildungschancen im gegliederten Schulsystem: Vertiefte PISA-Analyse unter Einbezug der kognitiven Grundfähigkeiten. Schweizerische Zeitschrift für Bildungswissenschaften, 25, 23-58.

Schellenberg, C., \& Häfeli, K. (2009). Erfolgsfaktoren beim Übergang von der Schule ins Berufsleben bei Jugendlichen mit ungünstigen Startchancen. Schweizerische Zeitschrift für Heilpädagogik, 15, 31-37.

Tracy, R. (2005). Spracherwerb bei vier- bis achtjährigen Kindern. In T. Guldimann \& B. Hauser (Hrsg.), Bildung 4- bis 8-jähriger Kinder (S. 59-75). Münster: Waxmann.

Weiss, R., \& Osterland, J. (1997). Grundintelligenztest Skala 1 - CFT. Göttingen: Hogrefe.

Wößmann, L. (2005). Ursachenkomplexe der PISA-Ergebnisse: Untersuchungen auf Basis internationaler Mikrodaten (IFO Working Paper No. 16). München: Institut für Wirtschaftsforschung.

Zöller, I., Roos, J., \& Schöler, H. (2006). Einfluss soziokultureller Faktoren auf den Schrftspracherwerb im Grundschulalter. In A. Schründer-Lenzen (Hrsg.), Risikofaktoren kindlicher Entwicklung: Migration, Leistungsangst und Schulübergang (S.45-65). Wiesbaden: VS Verlag für Sozialwissenschaften.

Open Access Dieses Kapitel wird unter der Creative Commons Namensnennung 4.0 International Lizenz (http://creativecommons.org/licenses/by/4.0/deed.de) veröffentlicht, welche die Nutzung, Vervielfältigung, Bearbeitung, Verbreitung und Wiedergabe in jeglichem Medium und Format erlaubt, sofern Sie den/die ursprünglichen Autor(en) und die Quelle ordnungsgemäß nennen, einen Link zur Creative Commons Lizenz beifügen und angeben, ob Änderungen vorgenommen wurden.

Die in diesem Kapitel enthaltenen Bilder und sonstiges Drittmaterial unterliegen ebenfalls der genannten Creative Commons Lizenz, sofern sich aus der Abbildungslegende nichts anderes ergibt. Sofern das betreffende Material nicht unter der genannten Creative Commons Lizenz steht und die betreffende Handlung nicht nach gesetzlichen Vorschriften erlaubt ist, ist für die oben aufgeführten Weiterverwendungen des Materials die Einwilligung des jeweiligen Rechteinhabers einzuholen.

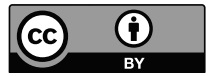


Übergänge in der Schule 


\section{Der FOKUS-Ansatz: Hyperaktive, impulsive und unaufmerksame Kinder nach der Einschulung fördern}

Sara Benini, Jennifer C. Fräulin und Markus P. Neuenschwander

\section{Zusammenfassung}

Unaufmerksamkeit, Hyperaktivität und Impulsivität sind frühe Indikatoren für einen gefährdeten Bildungsverlauf. Zur Förderung von Kindern mit diesen Verhaltensweisen im Grundschulunterricht wurde der FOKUS-Ansatz entwickelt. Der FOKUS-Ansatz enthält praktische pädagogische Massnahmen gegenüber Kindern mit einer Neigung zur ADHS Symptomatik im Unterricht - in Ergänzung zu allfälligen Medikamenten. Es wurden konkrete Elemente (1) einer wirksamen Klassenführung (Klassenebene), (2) des Umgangs mit einzelnen unaufmerksamen, hyperaktiven und impulsiven Kindern (individuelle Ebene) und (3) der Zusammenarbeit mit den Eltern dieser Kinder (Elternebene) wissenschaftsbasiert erarbeitet und in Zusammenarbeit mit Praktikerinnen konkretisiert. Zur Vermittlung des FOKUS-Ansatzes wurde eine Weiterbildung mit rund 90 Lehrpersonen durchgeführt. Eine Evaluation im Vergleich mit einer Kontrollgruppe zeigte, dass die Lehrpersonen wichtige Weiterbildungsinhalte umsetzten und dass dies zur Zunahme der Aufmerksamkeit und der sozialen Integration der Kinder in die Klasse geführt hat. Der Effekt auf die Hyperaktivität/Impulsivität war nur ansatzweise zu finden. Der FOKUS-Ansatz und die Weiterbildung haben sich als erfolgreich erwiesen und werden zukünftig für Lehrpersonen angeboten.

\section{Schlagworte}

Hyperaktivität/Impulsivität, Unaufmerksamkeit, Schule, Weiterbildung, Wirkungsstudie 


\section{$1 \quad$ Einleitung}

Mit der Einschulung beginnt für die Kinder ein neuer Lebensabschnitt, welcher Herausforderungen mit sich bringt. Dazu gehören unter anderem die Positionierung innerhalb des Klassenverbands, das Kennenlernen der Lehrperson/en sowie veränderte Tagesstrukturen. Dabei zeigt sich, dass manche Kinder grosse Mühe haben, lange still zu sitzen und konzentriert Aufgaben zu bearbeiten. Treten diese Verhaltensweisen besonders ausgeprägt und häufig auf, kann es nach gründlicher klinischer Abklärung zur Diagnostizierung einer Aufmerksamkeitsdefizit-Hyperaktivitätsstörung (ADHS) kommen. ADHS wird nach dem US-Amerikanischen Klassifikationsschema DSM-5 (APA, 2013) in zwei Kernsymptome unterteilt: a) Hyperaktivität/Impulsivität und b) Unaufmerksamkeit. Es wird beobachtet, dass hohe Ausprägungen von Hyperaktivität/Impulsivität und Unaufmerksamkeit ohne Behandlung ein Risiko für die zukünftige gesundheitliche sowie soziale und schulische Entwicklung der Betroffenen darstellen können. Hyperaktives/ impulsives Verhalten äussert sich anhand exzessiver Ruhelosigkeit sowie anhand von Ungeduld und Schwierigkeiten, etwas abwarten und Bedürfnisse aufschieben zu können. Unaufmerksames Verhalten in der Schule äussert sich anhand von häufigen, vorzeitigen Abbrüchen von Aktivitäten und unordentlicher, nachlässiger Arbeitsweise. Hyperaktivität/Impulsivität sowie Unaufmerksamkeit wirken sich in vielfältiger Art negativ auf die Situation in der Schule, das Lernen, die Leistungen und die Schulkarriere der Kinder aus (Barkley, 2006, Döpfner, 2001). Verhaltensauffällige Kinder zeigen tiefere Leistungen als ihre Mitschülerinnen und Mitschüler, obwohl sie aufgrund ihrer kognitiven Fähigkeiten im Unterricht durchschnittliche Leistungen erbringen könnten (Döpfner, Frölich \& Lehmkuhl, 2013). Weiter zeigte eine Studie, dass Kinder und Jugendliche mit ADHS häufiger als ihre Mitschülerinnen und Mitschüler Schultypen mit Grundanforderungen oder kürzere Berufsausbildungen besuchen (Bader, Pierrehumbert, Junier \& Halfon, 2005). Neuere Untersuchungen konnten direkte Auswirkungen von Verhaltensproblemen im Unterricht auf die Übertrittsentscheidungen in die Sekundarstufe I nachweisen (Neuenschwander \& Malti, 2009). Langzeitstudien weisen zudem darauf hin, dass verhaltensauffällige und unaufmerksame Kinder ein höheres Risiko haben, in Sonderschulen platziert zu werden. Mit grösserer Wahrscheinlichkeit riskieren sie ihre Ausbildung vorzeitig abzurechen oder Berufsausbildungen mit niedrigem Anforderungsniveau aufzunehmen (Barkley, Fischer, Edelbrock \& Smallish, 1991; Mannuzza, Klein, Bessler, Malloy \& Hynes, 1997).

Um den genannten langfristigen Beeinträchtigungen für den Schulverlauf vorzubeugen, sollte eine Intervention wegen der Situationsspezifität der Symptomatik und ihren vielfältigen Ausprägungen dort ansetzen, wo die Probleme auftreten 
(Döpfner, Schürman \& Frölich, 2002). Es sind demzufolge effektive Massnahmen erforderlich, die im Unterrichtsalltag einen nachgewiesenen positiven Effekt auf Verhaltensauffälligkeit und Unaufmerksamkeit haben. Diese Massnahmen sind auch bei einer klinisch nicht diagnostizierten ADHS notwendig und sollten von Lehrpersonen in der Regelklasse umgesetzt werden können. Es stellt sich somit die Frage, wie Kinder mit Neigung zu Unaufmerksamkeit und Hyperaktivität/Impulsivität im Unterricht nach der Einschulung besonders gefördert werden können.

\section{Der FOKUS-Ansatz}

Vor dem dargestellten Hintergrund wurde ein Konzept zu einem angemessenen Umgang der Lehrpersonen mit Schülerinnen und Schülern mit Verhaltens- und Aufmerksamkeitsproblemen im Unterricht und deren Eltern entwickelt, der FOKUS-Ansatz ${ }^{1}$ (Förderung von Kindern mit Verhaltensauffälligkeit und Unaufmerksamkeit in der Schule). In Abbildung 1 sind die dem FOKUS-Ansatz zugrunde gelegten Annahmen graphisch dargestellt.

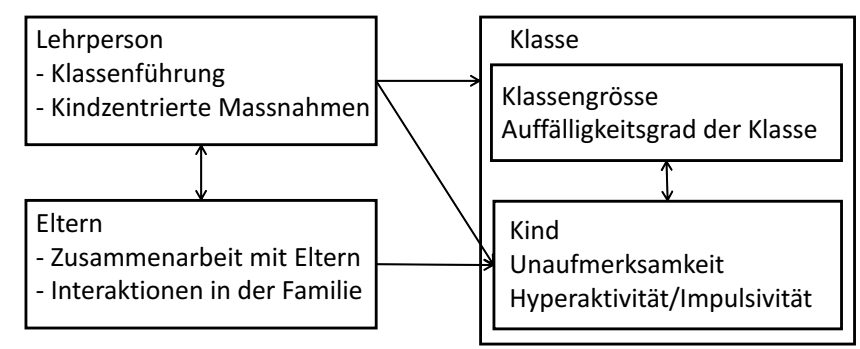

Abb. 1 Arbeitsmodell des FOKUS-Ansatzes

Es wird davon ausgegangen, dass Lehrpersonen durch eine sensibilisierte Einstellung und geeignete Handlungen die aufkommenden Schwierigkeiten erfolgreich und

1 Für eine detaillierte Beschreibung des FOKUS-Ansatzes und der Weiterbildung s. Neuenschwander, M. P. \& Benini, S. (2016). FOKUS - Förderung von Kindern mit Verhaltensauffälligkeiten und Unaufmerksamkeit im Unterricht. Schlussbericht z.Hd. des Bundesamtes für Gesundheit. Solothurn: Zentrum Lernen und Sozialisation der PH FHNW (www.fhnw.ch/ph/zls/fokus) 
nachhaltig bearbeiten und dadurch Schülerinnen und Schüler in ihrer schulischen Entwicklung fördern können. Auf der Ebene der Klassenführung sind eine hohe Strukturierung der Lerntätigkeit und des Lerngegenstandes (Klassenebene) sowie eine möglichst individuelle Anleitung des Kindes (individuelle Ebene) notwendig (Frölich, Döpfner \& Banaschewski, 2014; Zentall, 1993; Gawrilow, Guderjahn \& Gold, 2013). Um diesen Kindern eine erfolgreiche Mitarbeit in der Klassengemeinschaft zu ermöglichen, benötigt es seitens der Lehrpersonen einen strukturierenden und wertschätzenden Umgang. Damit kann auch bei den Mitschülerinnen und Mitschülern Verständnis und Akzeptanz geweckt werden.

Neben den Massnahmen, welche eine Lehrperson auf Klassenniveau umsetzen kann, sind gezielte, kindspezifische Massnahmen im Unterricht hilfreich. Es handelt sich um Massnahmen zur Aufmerksamkeitsförderung oder zur Förderung der Selbstregulation, welche den Kindern beim Denken, Planen und Handeln helfen. Das Ziel ist es, den Kindern zu helfen, ihre grundsätzlich vorhandenen Fähigkeiten in der richtigen Situation zum richtigen Zeitpunkt in Verhalten umzusetzen (Hoberg, 2013).

Darüber hinaus nimmt auch die Zusammenarbeit mit den Eltern der Schülerinnen und Schüler einen grossen Stellenwert ein (DuPaul et al., 2011; Mautone, Lefler \& Power, 2011). Eine gelingende Zusammenarbeit basiert auf drei Prinzipien: Austausch von Informationen, Aufbau von Vertrauen und Koordination von Massnahmen (Neuenschwander et al., 2005).

\subsection{Konzeption der Weiterbildung}

Ausgehend vom FOKUS-Ansatz wurde eine Weiterbildung zu folgenden drei Elementen der pädagogischen Förderung verhaltensauffälliger und unaufmerksamer Kinder im Unterricht erarbeitet (Neuenschwander \& Benini, 2017/im Druck): a) klassenzentrierte Massnahmen (Klassenführung), b) kinderzentrierte Massnahmen, insbesondere die Förderung der Selbstregulation, und c) förderliche Zusammenarbeit mit den Eltern. Die Weiterbildung richtet sich an Lehrpersonen der Unterstufe (1. und 2. Klasse). In Abbildung 2 ist die inhaltliche Struktur der FOKUS-Weiterbildung zusammenfassend dargestellt. Bei der Entwicklung der Weiterbildung wird das Schwergewicht auf wissenschaftlich überprüfte und wirksame pädagogische Strategien zur Förderung der Kinder gelegt, welche sinnvoll kombiniert werden, so dass sich am ehesten ein Erfolg für die Lehrpersonen und die Kinder einstellt. 


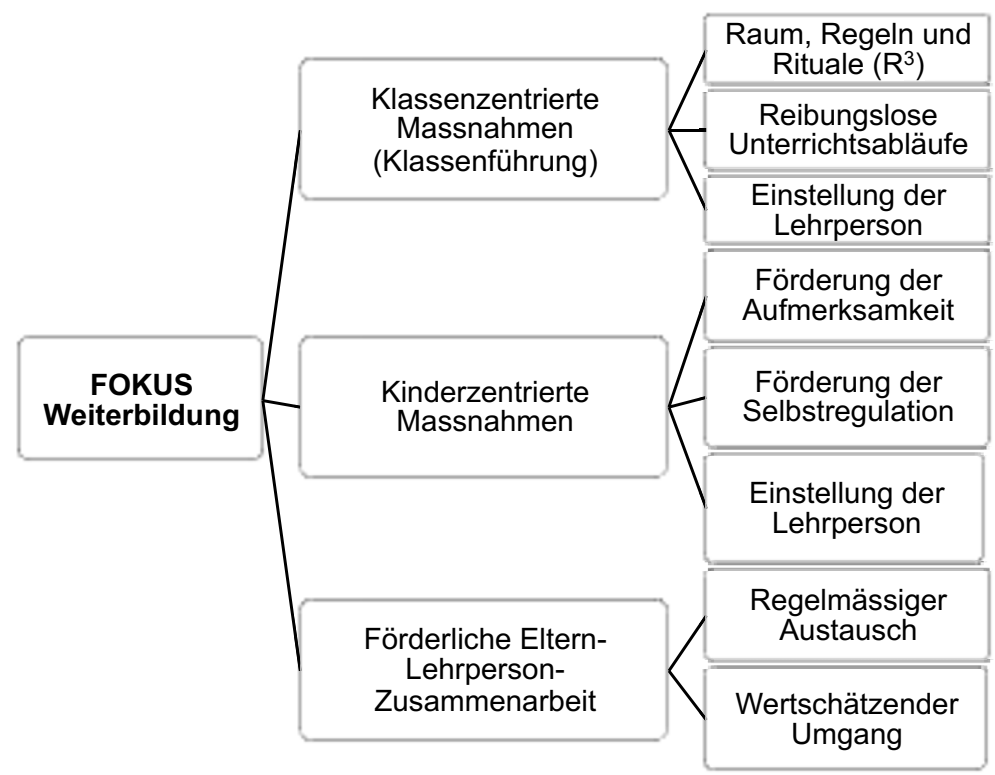

Abb. 2 Inhaltliche Struktur der FOKUS-Weiterbildung

Die Weiterbildung für Lehrpersonen ist so aufgebaut, dass zu jedem der drei Elemente des FOKUS-Ansatzes jeweils ein kurzer Theorieinput gegeben und eine themenspezifische Praxis-Checkliste verteilt wird. Danach werden konkrete Anwendungsbeispiele und Materialien im Plenum oder innerhalb kleiner Gruppen eingeführt und bearbeitet. Für die Wirksamkeit der Weiterbildung ist es wesentlich, dass Lehrpersonen die Elemente der Weiterbildung selbstständig und ohne grossen Aufwand im Unterricht einsetzen können (Gawrilow et al., 2013). Die Weiterbildung ist deshalb so konzipiert, dass sie sich über einen längeren Zeitraum erstreckt und dass die Lehrpersonen zwischen den Schulungen dank der zeitlichen Abstände (mind. ein Monat) immer die Möglichkeit zur Erprobung der Fortbildungsinhalte haben.

\subsection{Klassenzentrierte Massnahmen}

Lehrpersonen unterrichten erfolgreich, wenn sie klare Verhaltenserwartungen an die Kinder formulieren und ihnen Strukturierungshilfen bereitstellen (Jarman, 1996, Kos, Richdale \& Hay, 2006). Ein wesentlicher Aspekt dieses Teils der Wei- 
terbildung ist somit die Anregung der Selbstreflexion zur eigenen Klassenführung und zur Qualität des Unterrichts. Als Mittel zur "wertschätzenden Strukturierung“ des Unterrichts wird das Element Raum-Regeln-Rituale (R3) entwickelt und in die Weiterbildung eingeführt. Dabei stehen präventiv-proaktive Massnahmen im Vordergrund. In Bezug auf die Raumstrukturierung wird insbesondere darauf geachtet, dass im Klassenzimmer allfällige Reizüberflutungen auf ein Minimum reduziert werden. Bereits ab den ersten Wochen nach der Einschulung spielen bei der Strukturierung des Unterrichtsgeschehens, geeignete Klassenregeln und ritualisierte Unterrichtsabfolgen ebenfalls eine wichtige Rolle. Die Lehrpersonen werden im Rahmen der Weiterbildung auch für die selbsterfüllende Wirkung von Erwartungen sensibilisiert. Mittels Beispielen erfolgreicher Bildungsverläufe verhaltensauffälliger Kinder, akkurater Informationen zu Verhaltensauffälligkeiten und Unaufmerksamkeit im Kindesalter und Hinweisen sowie Erläuterungen zur Wirkung der Lehrpersoneneinstellung auf die Kinder wird das Bewusstsein der Lehrpersonen für diese Angelegenheit erweitert und verstärkt.

\subsection{Kinderzentrierte Massnahmen}

Zusätzlich zu allgemeinen Massnahmen sind individuelle, spezifische Interventionen beim Kind notwendig (DuPaul \& Stoner, 2003). Individuelle Massnahmen haben den Vorteil, dass sie auf die spezifische Situation des Kindes abgestimmt werden können. Sie setzen aber voraus, dass individualisiert unterrichtet wird. Bewegung gehört im Kindesalter zu einem gesunden Alltag. Den Lehrpersonen werden unterschiedliche Möglichkeiten vorgestellt, wie sie koordinative Bewegungsaufgaben bei Lern- und Wahrnehmungsvorgängen einbauen können. Weil das aufmerksame Zuhören eine der grundlegenden Aufgaben der Schülerinnen und Schüler in der Schule ist und Anleitungen von Arbeitsaufträgen im Unterricht in der Regel mündlich (d.h. akustisch) erteilt werden, wird den Lehrpersonen zur Aufmerksamkeitsförderung auch ein vom Projektteam selbst entwickeltes akustisches Konzentrationstraining (Ein FOKUS auf Ohr) vorgestellt. Die Lehrpersonen führen die Übungen zwei bis drei Mal in der Woche mit dem hyperaktiven/impulsiven und/oder unaufmerksamen Kind durch. Zur erfolgreichen Förderung und Unterstützung der Selbstregulation der Kinder bieten Wenn-Dann-Pläne eine effektive Lösung (Gawrilow, Schmitt \& Rauch, 2011; Gawrilow, Gollwitzer \& Oettingen, 2011). Den Lehrpersonen werden konkrete Beispiele und Materialien präsentiert, wie Wenn-Dann-Pläne erfolgreich im Unterricht eingesetzt werden können. 


\subsection{Förderliche Eltern-Lehrperson-Zusammenarbeit}

Um einen regelmässigen Kontakt mit den Eltern des Schülers oder der Schülerin zu pflegen, wird den Lehrpersonen die Wochenkarte vorgestellt, welche im $\mathrm{Zu}$ sammenhang mit der Durchführung der Wenn-Dann-Pläne eingesetzt werden kann. Die Lehrperson notiert auf der Karte, welcher Wenn-Dann-Plan das Kind gerade durchführt und wie die Umsetzung während der Woche gelaufen ist. Zum wertschätzenden Umgang mit dem verhaltensauffälligen Kind notiert die Lehrperson immer auch ein Lob der Woche. Zusätzlich werden die Lehrpersonen in das ElternInfoBlatt eingeführt. Dabei handelt es sich um ein Instrument zur Kommunikation einer ausführlichen Situationsbestimmung über das kindliche Verhalten in der Klasse, welches die Lehrpersonen drei bis vier Mal im Jahr ausfüllen und mit den Eltern persönlich besprechen. Schliesslich werden den Lehrpersonen gezielte Techniken der förderlichen Gesprächsführung mit Eltern vorgestellt und mit ihnen im Rahmen der Schulung geübt.

\subsection{Fragestellungen}

Vor diesem Hintergrund wurde in der vorliegenden Studie die Bearbeitung von Verhaltens- und Aufmerksamkeitsproblemen in der Schule untersucht. Die folgenden Forschungsfragen wurden verfolgt: a) Wie können Lehrpersonen im Hinblick auf die Optimierung ihrer Klassenführung effektiv weitergebildet werden? b) Wie verändert sich der Umgang der Lehrperson mit verhaltensauffälligen Kindern aufgrund der Weiterbildung? c) Können Unaufmerksamkeit und impulsive sowie hyperaktive Verhaltensweisen von Kindern des 2. Schuljahres durch eine konzeptbasierte Klassenführung, ergänzt mit kindspezifischen Massnahmen, reduziert werden?

Es wird demzufolge geprüft, ob eine eigens dafür entwickelte Intervention in Form einer Lehrpersonenweiterbildung einen positiven Effekt auf die Förderung von Verhaltensauffälligkeiten und Unaufmerksamkeit im Unterricht hat. Aus Platzgründen wird auf das Element der Elternzusammenarbeit im Folgenden nicht näher eingegangen. 


\section{$3 \quad$ Methode}

\subsection{Teilnehmende Lehrpersonen (Stichprobe)}

Die Stichprobe setzte sich aus Volksschullehrpersonen der 1.-2. Klasse aus neun Deutschschweizer Kantonen (67.9\% der Schulen in ländlichen Gebieten) zusammen. Das Durchschnittsalter der Lehrpersonen ( $94.9 \%$ weiblich) betrug $M=42.36$ Jahre $(S D=12.39)$ bei einer durchschnittlichen Berufserfahrung von $M=15.92$ Jahren $(S D=10.85)$. Aus jeder Klasse wurde jenes Kind in die Stichprobe miteinbezogen, dessen Eltern einer Teilnahme an der Studie zugestimmt hatten und welches gemäss dem ADHS-Index die höchste Ausprägung des Auffälligkeitswerts aufwies (Kapitel 3.3.1). Die teilnehmenden Kinder (79.6\% männlich) waren im Durchschnitt $M=8.65$ Jahre alt $(S D=1.02)$. Von den 137 Lehrpersonen, welche an der ersten Situationsanalyse (Pretest) teilgenommen hatten, waren an der zweiten Situationsanalyse (Posttest) noch 133 Lehrpersonen bei der Studie dabei. Insgesamt wurden die Angaben von vier Lehrpersonen aus den Analysen ausgeschlossen, weil sie im Pre- und/oder im Posttest den höchsten Belastungswert aufwiesen. Die Messwerte dieser Lehrpersonen wurden als sogenannte Ausreisser behandelt, welche zugunsten der Verlässlichkeit der Ergebnisse nicht in die Analysen einbezogen wurden. Wie frühere Studien zeigten, kann eine sehr hohe Belastung nämlich zu einer verzerrten Beurteilung des Schülerverhaltens führen. So beschrieben zum Beispiel Maslach und Jackson (1981) das Burnout-Syndrom bei stark belasteten Lehrpersonen unter anderem mit einer veränderten Schülerwahrnehmung (vgl. auch Heim \& Nido, 2008). Die Lehrpersonen und ihre Klassen wurden in drei Gruppen (Gruppe A, Gruppe AB und Kontrollgruppe) aufgeteilt. Diese unterschieden sich nicht signifikant in Bezug auf folgende Merkmale: Geschlecht, Durchschnittsalter der Lehrpersonen, durchschnittliche Berufserfahrung, Klassengrösse, Anteil Schülerinnen und Schüler mit Migrationshintergrund sowie Auffälligkeitswert des in die Studie einbezogenen Kindes.

\subsection{Durchführung}

Die Lehrpersonen wurden postalisch über die Schulleitung um eine Teilnahme an der Studie angefragt. Nachdem für jede teilnehmende Lehrperson die unterschriebenen Elterneinverständniserklärungen der Schülerinnen und Schüler vorlagen, wurde das Zielkind ausgewählt (Kapitel 2.3.1). Es fanden zwei Situationsanalysen statt. Die erste (Pretest) fand zwischen April und Juni 2014, die zweite (Posttest) zwischen Januar und April 2015 statt. Dabei wurde das Zielkind jeweils während 
einer Schullektion im Unterricht beobachtet, und die Lehrperson füllte einen Onlinefragebogen aus. Aufgrund des quasi-experimentellen Designs wurden die Lehrpersonen in drei Gruppen aufgeteilt: zwei Experimentalgruppen (A und $\mathrm{AB})$ und eine Kontrollgruppe. Lehrpersonen der Gruppe A wurden lediglich in den klassenzentrierten Massnahmen weitergebildet, während die Lehrpersonen der Gruppe AB zusätzlich zu kinderzentrierten Massnahmen und förderlicher Eltern-Lehrperson-Zusammenarbeit weitergebildet wurden (Kapitel 1.1). Lehrpersonen der Kontrollgruppe besuchten hingegen keine Weiterbildung. Zwischen den Situationsanalysen nahmen die Lehrpersonen der Experimentalgruppen im Juni, November und Dezember 2014 an den FOKUS-Weiterbildungen teil. Im September erhielten sie ein individuelles Coaching durch eine der beiden Weiterbildnerinnen.

\subsection{Instrumente}

\subsubsection{ADHS-Index}

In dieser Studie wurde nicht klinisch gearbeitet. Bei der Beurteilung des kindlichen Verhaltens hat man sich jedoch an den Dimensionen von ADHS orientiert. Die Lehrpersonen wurden gebeten für jedes Kind dessen Hyperaktivität, Impulsivität und Unaufmerksamkeit einzuschätzen. Zur Einschätzung verwendeten sie ein dafür vorgesehenen Instrument. Jede Lehrperson füllte somit für ihre Klasse einen ADHS-Index für Lehrpersonen (Lidzba, Christiansen, Drechsler, 2013) aus. Der Index besteht aus 10 Aussagen zu den Verhaltensweisen des Kindes innerhalb des letzten Monates (z. B. «Zappelt oder rutscht auf dem Stuhl herum»). Die Lehrperson sollte dabei angeben, wie gut diese Aussagen den jeweiligen Schüler/die jeweilige Schülerin beschreiben ( 0 ,traf überhaupt nicht auf das Kind zu' bis 3 ,traf ganz genau auf das Kind zu'). Für die Auswertung wurden die Antworten aufsummiert und in Abhängigkeit von Alter und Geschlecht ein Auffälligkeitswert (1, nicht auffällig' bis 4 ,sehr auffällig') bestimmt (Pretest: $M=3.69$ Punkte; $S D=.73$; Posttest: $M=2.55$ Punkte; $S D=1.10$ ). In jeder Klasse wurde jenes Kind mit dem höchsten Auffälligkeitswert in die Studie aufgenommen.

\subsubsection{Verhaltensbeobachtung}

Jedes Kind wurde während einer Lektion von einem Projektmitarbeitenden beobachtet. Dabei wurden mittels eines Beobachtungsrasters das methodisch didaktische Setting, die Verhaltensweisen des Kindes sowie die Lehrpersonenreaktionen darauf pro Minute festgehalten. Den geschulten Beobachtenden lag dabei eine standardisierte, schriftliche Instruktion vor. Eine Audiodatei, welche über einen Kopfhörer 
abgehört wurde, diente der Zeiteinteilung. Zudem sassen die Beobachtenden so, dass sie das Kind frontal oder seitlich sehen konnten. Für jede Beobachtungseinheit (1 Minute) wurden das Verhalten des Kindes anhand von vier Unterkategorien (,Regelverhalten', ,Unaufmerksamkeit', ,Hyperaktivität' und ,Impulsivität') kodiert. Gleichzeitig wurde die Lehrpersonenreaktion (,Lehrperson (LP) reagiert nicht auf das Verhalten des Kindes', ,LP lobt das Kind', ,LP erteilt eine Zurechtweisung', ,LP droht mit einer Sanktion', ,LP verfügt eine Massnahmec, ,LP verlegt den Arbeitsplatz des Kindes') darauf festgehalten. Pro Minute konnten maximal vier verschiedene Verhaltenskategorien der Kinder und die darauffolgende Reaktion kodiert werden. Pro Verhalten des Kindes wurde eine Lehrpersonenreaktion kodiert. Die Inter-Rater-Reliabilität (Cohens $K$ ) dieser Beobachtungen liegen zwischen .67 und .99 $(M=.80 ; S D=.11)$, was als eine gute bis fast vollkommene Übereinstimmung erachtet wird (Bortz \& Döring, 2005). Bei den Auswertungen wurde zwischen nonverbalen (z. B. Blickkontakt, positive Zurechtweisung) und individualisierenden (z. B. neuer Arbeitsauftrag oder neuer Arbeitsplatz) förderlichen Reaktionen unterschieden. Diese Reaktionen der Lehrperson auf bestimmte Verhaltensweisen wurden über die Lektion addiert und am Total aller Reaktionen gewichtet.

\subsubsection{Weiterbildungsevaluation}

Nach der letzten Schulung bewerteten die Lehrpersonen der Experimentalgruppen mittels Fragebogen unter anderem die einzelnen Elemente des FOKUS-Ansatzes ( 1 , gar nicht hilfreich' bis 6 , ausserordentlich hilfreich').

\subsubsection{Lehrpersonenfragebogen}

Im Onlinelehrpersonenfragebogen wurden unter anderen nachfolgende Konstrukte erhoben:

Mittels des Items „Haben Sie für dieses Schuljahr die Klassenregeln verändert und/oder ergänzt?» mit dichotomem Antwortformat wurden die Änderungen oder Anpassungen der Klassenregeln erhoben. Zur Erhebung der Strukturierung bestimmter Unterrichtsabläufe wurden fünf selbstkonstruierte Aussagen (z. B. „Jeden Morgen erkläre ich vor dem Unterrichtsbeginn den Plan des ganzen Tages anhand einer immer gleichen Darstellungsart.") eingesetzt, welche die Lehrpersonen mit ,trifft zu' oder , trifft nicht zu' beantworteten. Dabei wurden die Anzahl, trifft zu'-Antworten aufsummiert. Die Wertschätzung individueller Bedürfnisse der Kinder wurden auf einer 6er Skala ( 1 ,stimmt überhaupt nicht ' bis 6 ,stimmt voll und ganz') mittels sechs Items (z. B. „Ich gehe oft auf die Gefühle und Empfindungen der Kinder ein.") erhoben, welche zu einem Faktor zusammengefasst wurden ( $\alpha=$ .74). In Anlehnung an die American Psychiatric Association (APA, 2013) schätzte 
die Lehrperson das Verhalten des Kindes in Bezug auf die Unaufmerksamkeit (neun Items z. B. „Es kommt regelmässig vor, dass das Kind nur schwer seine Aufmerksamkeit aufrechterhalten kann.“; $\alpha=.81$ ) und auf die Hyperaktivität/Impulsivität (neun Items z. B. „Es kommt regelmässig vor, dass das Kind mit Händen und Füssen zappelt oder sich auf dem Stuhl windet.“; $\alpha=.92)$ auf einer 6er Skala ( 1 , stimmt überhaupt nicht ' bis 6 , stimmt voll und ganz') ein. Mit drei Items (z. B. „Das Kind ist bei anderen Kindern beliebt.") wurde in Anlehnung an Goodman (1997) die soziale Integration des Kindes im Klassenverband erhoben. Die Interne Konsistenz betrug $\alpha=.81$.

\section{$4 \quad$ Ergebnisse}

Die Wirkung der Weiterbildung wurde summativ überprüft. Es wurden die Mittelwerte zwischen den Gruppen und/oder zwischen Pre- und Posttest auf signifikante Unterschiede untersucht. Aufgrund der geringen Stichprobengrösse sind die inferenzstatistischen Berechnungen mit den Signifikanzniveaus 10\% und 5\% berechnet worden.

Damit eine Weiterbildung die erwartete Wirkung zeigen kann, ist es wichtig, dass Lehrpersonen die Inhalte dieser Weiterbildung im Unterricht umsetzen. Die Lehrpersonen beurteilen die Inhalte der FOKUS-Weiterbildung als, eher hilfreich ' bis , hilfreich'. Die Lehrpersonen, welche eine Weiterbildung besucht haben, haben die Kursinhalte in ihrem Unterricht eingeführt und umgesetzt.

\subsection{Wirkung auf das Verhalten der Lehrpersonen}

Bezüglich der Klassenführung zeigt sich, dass die Lehrpersonen nach der Weiterbildung im Vergleich zur Kontrollgruppe ihre Klassenregeln geändert oder angepasst haben: $\mathrm{X}^{2}(2,130)=22.1, p<.001$, Cramer's $V=.40$. Zudem strukturieren Lehrpersonen der Gruppen A und AB (Weiterbildungsgruppen) im Posttest signifikant mehr Unterrichtsabläufe als im Pretest und im Vergleich zu Lehrpersonen der Kontrollgruppe (Abbildung 3). Die Befunde zeigen zwischen Pre- und Posttest einen signifikanten Anstieg der Anzahl ritualisierter Abläufe im Unterricht auf: $F(1,123)=90.81, p<.01, \eta^{2}=.43$. Aufgrund der Varianzanalyse kann auch eine signifikante Wechselwirkung zwischen dem Messzeitpunkt und der Gruppenzugehörigkeit festgestellt werden: $F(2,123)=4.40, p<.05$. Dies deutet darauf hin, dass beim zweiten Messzeitpunkt nicht nur mehr Unterrichtsabläufe ritualisiert worden 
sind, sondern dass in den Weiterbildungsgruppen die Zunahme der Ritualisierung signifikant ausgeprägter ist als in der Kontrollgruppe.

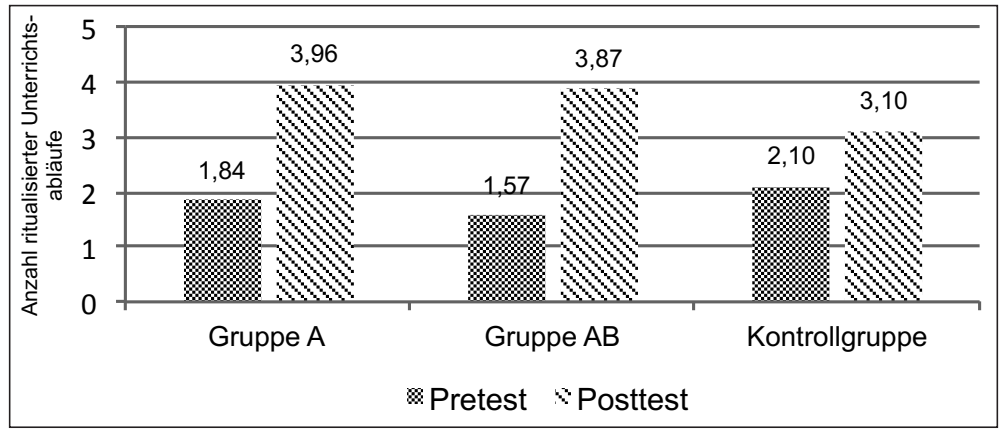

Abb. 3 Anzahl (1 bis 5) ritualisierter Unterrichtsabläufe (Varianzanalysen mit Messwiederholung)

Die Befunde zur berichteten Wertschätzung gegenüber den verhaltensauffälligen und unaufmerksamen Schülerinnen und Schülern (Abbildung 4) zeigen zwischen Pre- und Posttest bei den Weiterbildungsgruppen (A und AB) einen signifikanten Anstieg der Wertschätzung: $F(1,123)=6.18, p<.05, \eta^{2}=.05$. Die Varianzanalyse ergibt zudem einen signifikanten Interaktionseffekt zwischen der Weiterbildungsgruppe und dem Messzeitpunkt: $F(2,123)=3.04, p<.05, \eta^{2}=.05$. Obwohl die Effekte klein sind, bedeutet dies dennoch, dass in den Gruppen $\mathrm{A}$ und $\mathrm{AB}$ die Wertschätzung im Vergleich zur Kontrollgruppe gestiegen ist.

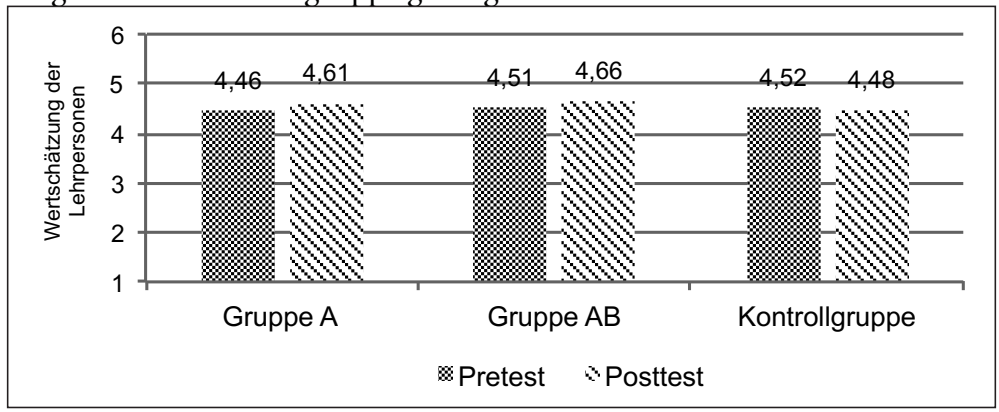

Abb. 4 Wertschätzung (Ausprägungsskala 1 bis 6) gegenüber verhaltensauffälligen und unaufmerksamen Schülerinnen und Schülern (Varianzanalysen mit Messwiederholung) 
Bei den dargestellten Ergebnissen wird der Schwerpunkt auf förderliche Reaktionen der Lehrpersonen gelegt. Anhand der in der Grafik dargestellten Daten (Abbildung 5) ist ersichtlich, dass der Anteil nonverbaler förderlicher Reaktionen auf Hyperaktivität/Impulsivität der Lehrpersonen in den Gruppen A und AB im Vergleich zum Pretest im Posttest grösser ist, wenn auch nicht signifikant: $F(1,115)=1.99$, n.s., $\eta^{2}=.02$. Jedoch unterscheidet sich die Veränderung des Anteiles förderlicher nonverbaler Reaktionen auf hyperaktive/impulsive Verhaltensweisen bei den Weiterbildungsgruppen im Vergleich zur Kontrollgruppe signifikant. Der relative Anteil nonverbaler förderlicher Reaktionen an allen beobachteten Reaktionen bei den Lehrpersonen der Gruppen A und AB ist im zweiten Messzeitpunkt grösser als jener der Kontrollgruppe, welcher in der Tendenz eher abgenommen hat: $F(2,69)=$ 3.96, $p<.05, \eta^{2}=.10$. Im Vergleich dazu weisen die Ergebnisse bezüglich nonverbaler förderlicher Reaktionen auf Unaufmerksamkeit zwischen den Weiterbildungsgruppen und der Kontrollgruppe weder im Zeitvergleich: $F(1,115)=1.99$, n.s., $\eta^{2}=.02$, noch in der Wechselwirkung zwischen Zeit und Gruppenzugehörigkeit: $F(2,123)=$ .29 , n.s., $\eta^{2}=.01$ auf signifikante Veränderungen hin.

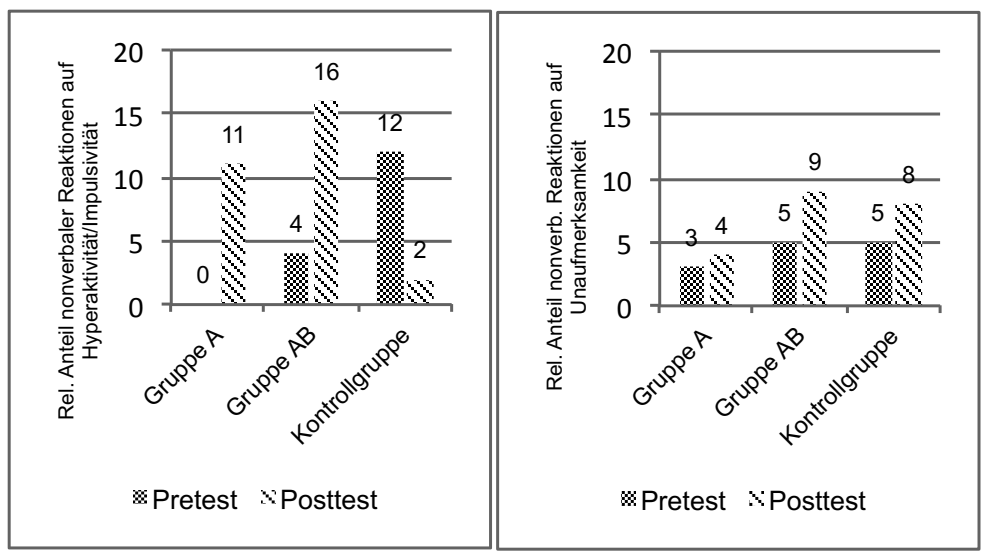

Abb. 5 Anteil beobachteter, nonverbaler förderlicher Reaktionen der Lehrperson auf Unaufmerksamkeit und Hyperaktivität/Impulsivität (Varianzanalysen mit Messwiederholung)

In Abbildung 6 sind die Auswertungen für individualisierende förderliche Reaktionen der Lehrpersonen auf hyperaktive/impulsive und unaufmerksame Verhaltensweisen dargestellt. Anhand der Daten ist ersichtlich, dass Lehrpersonen Hyperaktivität/ 
Impulsivität und Unaufmerksamkeit seltener mit individualisierenden Reaktionen als mit nonverbalen Reaktionen begegnen. Der Vergleich zwischen Pretest und Postest weist auf eine Zunahme förderlicher Reaktionen auf Unaufmerksamkeit hin, welche jedoch nicht signifikant ausfällt: $F(1,115)=.02$, n.s., $\eta^{2}=.00$. Beim Vergleich der Gruppen ist ersichtlich, dass bei den Weiterbildungsgruppen A und $\mathrm{AB}$ der Anteil individualisierender förderlicher Reaktionen auf Unaufmerksamkeit im Vergleich zur Kontrollgruppe, bei welcher dieser Anteil eher abgenommen hat, gestiegen ist. Der Interaktionseffekt ist für Unaufmerksamkeit signifikant: $F(2$, $115)=3.5, p<.05, \eta^{2}=.06$. Lehrpersonen der Weiterbildungsgruppen reagieren öfters als Lehrpersonen der Kontrollgruppe mit individualisierenden förderlichen Reaktionen. Was hingegen die individualisierenden Reaktionen auf Hyperaktivität/ Impulsivität betrifft, wurden weder im Zeitvergleich: $F(1,69)=.42$, n.s., $\eta^{2}=.01$, noch im Interaktionseffekt: $F(2,69)=1.38$, n.s., $\eta^{2}=.04$ signifikante Unterschiede gefunden.

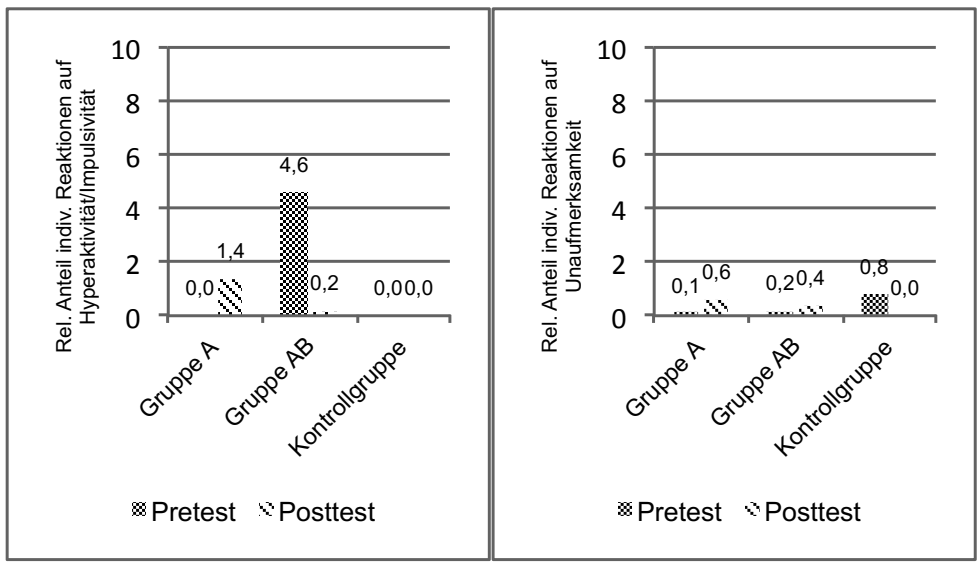

Abb. 6 Anteil beobachteter nonverbaler förderlicher Reaktionen der Lehrperson auf Unaufmerksamkeit und Hyperaktivität/Impulsivität (Varianzanalysen mit Messwiederholung)

\subsection{Wirkung auf das Verhalten des Zielkindes}

Bezüglich der Situation des Zielkindes in der Klasse, gemessen an der von der Lehrperson eingeschätzten sozialen Integration im Klassenverband, weist der Vergleich zwischen Pre- und Posttest auf eine tendenziell erwünschte Entwicklung 
hin (Abbildung 7). Die Veränderung ist nicht signifikant: $F(1,123)=.43$, n.s., $\mathrm{\eta}^{2}=$ .00. Die Varianzanalyse mit Messwiederholung weist aber bezüglich der sozialen Integration eine signifikante Wechselwirkung: $F(2,123)=3.16, p<.05, \eta^{2}=.05$, zwischen dem Messzeitpunkt und der Gruppenzugehörigkeit auf. Kinder, deren Lehrpersonen eine Weiterbildung besucht haben, sind bei der zweiten Messung sozial integrierter in ihrer Klasse als Kinder, deren Lehrperson keine Weiterbildung besucht hat.

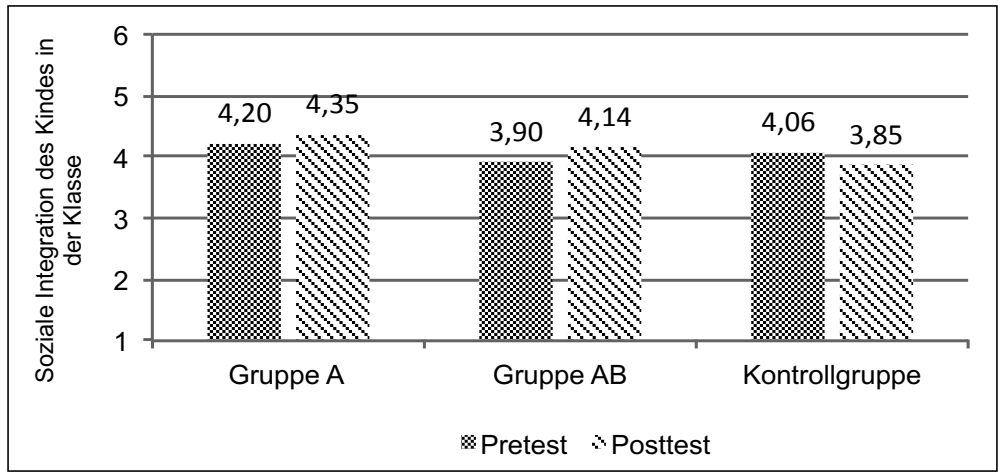

Abb. 7 Einschätzung (Ausprägung der Skala 1 bis 6) der Lehrpersonen zur sozialen Integration des Kindes in der Klasse (Varianzanalysen mit Messwiederholung)

Zudem wurde geprüft, ob sich die Verhaltensweisen der Kinder im Unterricht aufgrund der Weiterbildung positiv verändert haben (Abbildung 8). Anhand der Einschätzung der Lehrpersonen hat sowohl die Hyperaktivität/Impulsivität als auch die Unaufmerksamkeit während der Intervention gesamthaft, d. h. in allen drei Gruppen, abgenommen. Für beide Dimensionen wurden signifikante Veränderungen zwischen dem ersten und zweiten Messzeitpunkt gefunden: Hyperaktivität/Impulsivität: $F(1$, $123)=40.65, p<0.01, \eta^{2}=.25$ und Unaufmerksamkeit: $F(1,123)=109.28, p<0.01, \eta^{2}=.47$. 

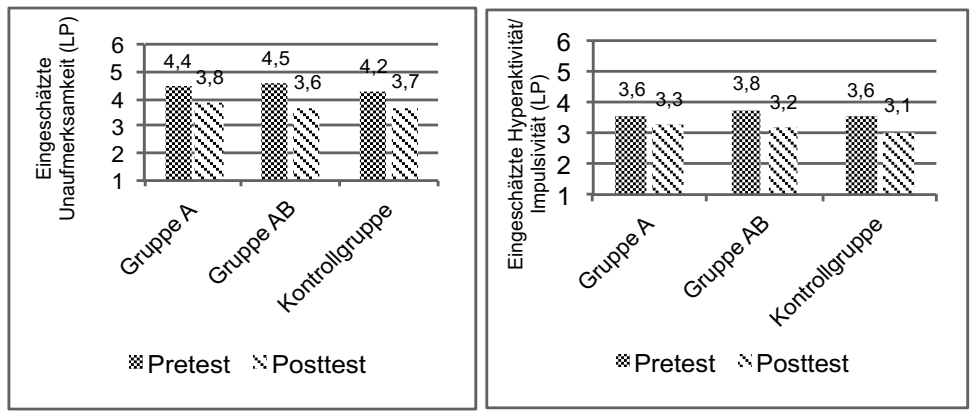

Abb. 8 Einschätzung (Ausprägung der Skala 1 bis 6) seitens der Lehrperson der Hyperaktivität/Impulsivität und Unaufmerksamkeit des Zielkindes während des letzten Monats vor der Erhebung (Varianzanalysen mit Messwiederholung)

Für Unaufmerksamkeit ergibt sich auf dem Zehn-Prozent-Niveau ein signifikanter Interaktionseffekt: $F(2,123)=2.74, p<0.1, \eta^{2}=.04$. Werden die einzelne Weiterbildungsgruppen mit der Kontrollgruppe verglichen, dann ergibt sich ein signifikanter Interaktionseffekt zwischen der Gruppe AB und der Kontrollgruppe: $F(1,74)=4.16$, $p<0.05, \eta^{2}=.05$. Bei den Kindern, deren Lehrpersonen die Weiterbildung zu allen drei Elementen des FOKUS-Ansatzes besucht haben, hat die Unaufmerksamkeit stärker abgenommen als in der Kontrollgruppe. Obwohl die Daten zur Hyperaktivität/Impulsivität eine ähnliche Tendenz aufzeigen, konnte keine signifikante Interaktion ermittelt werden (Tabelle 1). 
Tab. 1 Gruppenvergleich der Einschätzung seitens der Lehrperson der Hyperaktivität/ Impulsivität und Unaufmerksamkeit des Zielkindes während des letzten Monats vor der Erhebung (Varianzanalysen mit Messwiederholung)

\begin{tabular}{|c|c|c|c|}
\hline & Gruppe A & Gruppe AB & Kontrollgruppe \\
\hline \multicolumn{4}{|l|}{ Unaufmerksamkeit } \\
\hline Unaufmerksamkeit $t_{1}$ & $4.43(.70)$ & - & $4.23(.87)$ \\
\hline Unaufmerksamkeit $t_{2}$ & $3.83(.86)$ & - & $3.70(.96)$ \\
\hline $\mathrm{F}, \mathrm{df}_{1}, \mathrm{df}_{2}, \mathrm{p}, \mathrm{R}^{2}$ & $\begin{array}{l}Z: 68.92^{\star * *}, 1,87 \\
\eta^{2}=.44\end{array}$ & $\begin{array}{l}\text { G: } 1.01,1,87, \\
\eta^{2}=.01\end{array}$ & $\begin{array}{l}\text { I: . } 31,1,87, \\
\eta^{2}=.00\end{array}$ \\
\hline Unaufmerksamkeit $t_{1}$ & - & $4.53(.72)$ & $4.23(.87)$ \\
\hline Unaufmerksamkeit $t_{2}$ & - & $3.64(1.05)$ & $3.70(.96)$ \\
\hline $\mathrm{F}, \mathrm{df}_{1}, \mathrm{df}_{2}, \mathrm{p}, \mathrm{R}^{2}$ & $\begin{array}{l}Z: 63.13^{* * *}, 1,74 \\
\eta^{2}=.46\end{array}$ & $\begin{array}{l}\text { G: . } 45,1,74, \\
\eta^{2}=.01\end{array}$ & $\begin{array}{l}\text { I: } 4.16^{*}, 1,74, \\
\eta^{2}=.05\end{array}$ \\
\hline \multicolumn{4}{|c|}{ Hyperaktivität/Impulsivität } \\
\hline Hyperaktivität $\mathrm{t}_{1}$ & $3.58(1.29)$ & - & $3.55(1.29)$ \\
\hline Hyperaktivität $t_{2}$ & $3.26(1.25)$ & - & $3.05(1.29)$ \\
\hline $\mathrm{F}, \mathrm{df}_{1}, \mathrm{df}_{2}, \mathrm{p}, \mathrm{R}^{2}$ & $\begin{array}{l}Z: 29.08^{\star * *}, 1,87 \\
\eta^{2}=.25\end{array}$ & $\begin{array}{l}\mathrm{G}: .23,1,87, \\
\eta^{2}=.00\end{array}$ & $\begin{array}{l}\text { I: } 1.45,1,87, \\
\eta^{2}=.02\end{array}$ \\
\hline Hyperaktivität $\mathrm{t}_{1}$ & - & $3.76(1.05)$ & $3.55(1.29)$ \\
\hline Hyperaktivität $t_{2}$ & - & $3.16(1.13)$ & $3.05(1.29)$ \\
\hline $\mathrm{F}, \mathrm{df}_{1}, \mathrm{df}_{2}, \mathrm{p}, \mathrm{R}^{2}$ & $\begin{array}{l}Z: 28.47^{\star * *}, 1,74 \\
\eta^{2}=.28\end{array}$ & $\begin{array}{l}\text { G: } .41,1,74, \\
\eta^{2}=.01\end{array}$ & $\begin{array}{l}\text { I: } .25,1,74, \\
\eta^{2}=.00\end{array}$ \\
\hline
\end{tabular}

Legende. ${ }^{* *}: \mathrm{p}<.001,{ }^{* *}: \mathrm{p}<.01,{ }^{*}: \mathrm{p}<.05,+: \mathrm{p}<.10, \mathrm{G}$ : Gruppeneffekt, Z: Zeiteffekt, I: Interaktionseffekt Gruppe mal Zeit, $t_{1}$ : Pretest, $t_{2}$ : Posttest, Gruppe A: verkürzte WB, Gruppe $\mathrm{AB}$ : gesamte WB

\section{$5 \quad$ Schlussfolgerungen}

Hyperaktive/impulsive und unaufmerksame Verhaltensweisen im Unterricht können sich negativ auf die schulische Entwicklung der Kinder auswirken (Döpfner, Frölich \& Lehmkuhl, 2013; Barkley, 2006; Döpfner, 2001). Es gibt aber besondere Unterrichtsgestaltungen und Organisationsformen, von denen hyperaktive/impulsive und unaufmerksame Schülerinnen und Schüler profitieren (Frölich et al., 2014; Zentall, 1993; Gawrilow, Guderjahn \& Gold, 2013). Aus diesem Grund wurde ein Konzept entwickelt, wie verhaltensauffällige und unaufmerksame Schülerinnen und Schüler von den Lehrpersonen der Unterstufe angemessen und längerfristig gefördert werden können. Es wurde geprüft, ob die entwickelte Intervention, die FOKUS-Lehrpersonenweiterbildung, einen positiven Effekt im Hinblick auf die 
effektive Weiterbildung der Lehrpersonen und auf die Veränderung des Umgangs der Lehrpersonen mit verhaltensauffälligen Kindern erzielte. Weiter wurde untersucht, ob die FOKUS-Weiterbildung auf die Vorbeugung oder Reduzierung von Unaufmerksamkeit und impulsiven und hyperaktiven Verhaltensweisen von Kindern des zweiten Schuljahres einen Effekt hatte.

Gesamthaft zeigen die Ergebnisse, dass die Weiterbildung bei den Lehrpersonen auf Akzeptanz gestossen ist und die Lehrpersonen die meisten der vermittelten pädagogischen Strategien angewendet und umgesetzt haben. So nutzten sie beispielsweise die Formulare und Materialien für den Unterricht, die ihnen in der Weiterbildung gezeigt worden sind. Sie wenden aber auch die konkreten Hinweise zur Klassenführung, zur Ritualisierung des Unterrichts sowie zur Klassenzimmergestaltung an. Die erzielten Ergebnisse zeigen, dass Lehrpersonen der Unterstufe durch sehr konkret und direkt auf Anwendungen ausgerichtete Weiterbildungsinhalte erfolgreich, d.h. effektiv, weitergebildet werden können. Bereits in der Weiterbildungssituation wurden im Sinne des situierten Lernens (Mandl, 2004) Anwendungen diskutiert. Dadurch wurde die Verwendbarkeit des vermittelten Wissens und der Hilfsmittel im eigenen Unterricht vereinfacht.

Aufgrund der FOKUS-Weiterbildung haben die Lehrpersonen ihr Verhalten im Unterrichtsalltag verändert. Die Lehrpersonen der Weiterbildungsgruppen strukturieren die Lernumgebung und die Lehrtätigkeit angemessener zur Förderung von verhaltensauffälligen und unaufmerksamen Kindern in der Klasse. Sie achten auf wichtige Aspekte bei der Einführung von Klassenregeln und involvieren die Kinder stärker in den Erarbeitungsprozess derselben. Lehrpersonen, die eine Weiterbildung besucht haben, ritualisieren mehr Unterrichtsabläufe. Dadurch entstehen geeignetere und unterstützendere Lernbedingungen für verhaltensauffällige und unaufmerksame Kinder und die gesamte Klasse.

Die Weiterbildung wirkt sich nicht nur auf die Strukturierung des Unterrichts aus, sondern auch auf die Einstellung der Lehrpersonen gegenüber den auffälligen und unaufmerksamen Schülerinnen und Schülern und auf deren Umgang mit störendem Verhalten. Die Ergebnisse zeigen bezüglich der Wertschätzung der Lehrpersonen gegenüber auffälligen Kindern und ihrer Reaktionsweisen beim Auftreten von störenden Verhaltensweisen positive Veränderungen. Diese treten in der Lehrpersoneneinschätzung auf, werden aber auch in den Verhaltensbeobachtungen sichtbar. Lehrpersonen, die eine Weiterbildung besucht haben, reagieren bei Auftreten von Unaufmerksamkeit oder Hyperaktivität/Impulsivität anteilmässig häufiger mit förderlichen Reaktionen als Lehrpersonen der Kontrollgruppe, wo der Anteil positiver Reaktionen tendenziell abnimmt. Oftmals fallen Lehrpersonen meistens unabsichtlich - in eine negative Reaktionsspirale. Dabei folgen auf störende Verhaltensweisen negative und frustrierte Reaktionen der Lehrpersonen. Diese 
lösen wiederum Frustration beim Kind aus. Durch Letztere werden hyperaktive/ impulsive sowie unaufmerksame Verhaltensweisen verstärkt. Lehrpersonen der Weiterbildungsgruppen haben den Wechsel von der frustrierten, negativen Reaktion gegenüber dem Kind zu einer wertschätzenden, förderlichen Reaktion geschafft. Wird die negative Reaktionsspirale durchbrochen, nimmt auch die Häufigkeit von belastenden Situationen im Unterrichtsalltag ab.

Nebst auf das Verhalten der Lehrpersonen zeigt die Weiterbildung auch eine positive Wirkung a) auf die Situation der Kinder in der Klasse und b) ansatzweise auf ihr Verhalten im Unterricht. Aufgrund der Weiterbildung konnte die soziale Integration des Zielkindes in den Klassenverband im Vergleich zur Kontrollgruppe deutlich verbessert werden. Dabei handelt sich um einen wichtigen Effekt für die Förderung dieser Schülerinnen und Schüler in der Schule. Aus früheren Studien weiss man, dass verhaltensauffällige und unaufmerksame Kinder in der Klasse sozial weniger vorgezogen werden und grössere Schwierigkeiten haben, Freundschaften zu knüpfen und dauerhaft aufrechtzuerhalten (Hoza et al., 2005).

Die Ergebnisse belegen ferner eine positive Wirkung der Massnahmen auf die Unaufmerksamkeit und ansatzweise auf die Hyperaktivität/Impulsivität der Zielkinder. Auffällige und unaufmerksame Verhaltensweisen haben zwischen den beiden Messzeitpunkten abgenommen. Allerdings konnte nur bei den Lehrpersonen, welche die vollständige Weiterbildung besucht haben,nämlich zu allen drei Elementen des FOKUS-Ansatzes (Gruppe AB), eine Abnahme der Unaufmerksamkeit im Vergleich zur Kontrollgruppe belegt werden. Daraus lässt sich schliessen, dass für eine geeignete Förderung von verhaltensauffälligen und unaufmerksamen Kindern in der Klasse das umfassendere Weiterbildungskonzept erfolgreicher ist. Nebst einer angemessenen Klassenführung hat sich die Einführung gezielter kindspezifischer Fördermassnahmen in den Unterrichtsalltag bewährt.

Die Weiterbildungsinhalte und die Evaluationsergebnisse illustrieren, wie durch Lehrpersonenweiterbildung und mit pädagogischen Massnahmen die Situation von unaufmerksamen und hyperaktiven/impulsiven Kindern im Regelunterricht der Grundschule verbessert werden kann. Wegen der kleinen Stichprobe werden nur starke Effekte signifikant. Dadurch wird die Aussagekraft der Befunde zusätzlich erhöht. Die Ergebnisse belegen das grosse Potenzial des FOKUS-Ansatzes für die Bearbeitung von Unaufmerksamkeit und Hyperaktivität/Impulsivität bei Kindern auf der Unterstufe und für die Förderung deren sozialen Einbindung in die Klasse. Insbesondere dieser letzte Aspekt wird von den Lehrpersonen als sehr positiv zurückgemeldet. Durch gezielte pädagogische Interventionen, welche in der Schule von den Lehrpersonen umgesetzt werden können, kann davon ausgegangen werden, dass indirekt eine gewisse Sekundärprävention von Störungen und erschwerten Schulverläufen im Jugend- und Erwachsenenalter geleistet wird. 
Insgesamt findet man eine stärkere Wirkung der Weiterbildung auf Unaufmerksamkeit als auf Hyperaktivität/Impulsivität. Die Ergebnisse zeigen generell, dass sich die Bedingungen von Unaufmerksamkeit von den Bedingungen der Hyperaktivität/ Impulsivität unterscheiden. Entsprechend müssen zukünftig für beide Verhaltensweisen spezifische Massnahmen entwickelt und umgesetzt werden. In Bezug auf die Bearbeitung der Hyperaktivität/Impulsivität mit Hilfe des FOKUS-Ansatzes gibt es einen gewissen Optimierungsbedarf bei der Weiterbildung. Es müssen zukünftig weitere Strategien mit nachweisbarer Wirkung beim Umgang mit hyperaktiven/ impulsiven Kindern im Regelunterricht erarbeitet werden. Diese Erkenntnisse sind wichtig, um zukünftigen Analysen der Wirkung der FOKUS-Weiterbildung auch auf anderen Schulstufen (Kindergarten oder höhere Klassen der Primarschule) erfolgreich replizieren zu können.

\section{Literatur}

American Psychiatric Association (2013). Diagnostic and statistical manual of mental disorders: DSM-5. American Psychiatric Association (5. Auflage). Arlington, Va.: American Psychiatric Association.

Bader, M., Pierrehumbert, B., Junier, L., \& Halfon, O. (2005). Die Aufmerksamkeitsdefizit-Hyperaktivitäts-Störung bei Kindern und Jugendlichen. Bericht über eine 2001 in Morges durchgeführte Studie und eine Umfrage bei der Ärzteschaft des Kantons Waadt. Lausanne: BAG.

Barkley, R. A. (2006). Attention Deficit-Hyperactivity Disorder: A handbook for diagnosis and treatment (3. Aufl.). New York: Guilford.

Barkley, R. A., Fischer, M., Edelbrock, C., \& Smallish, L. (1991). The adolescent outcome of hyperactive children diagnosed by research criteria: III. Mother-child interactions, family conflicts and maternal psychopathology. Journal of Child Psychology and Psychiatry, 32, 233-256.

Bortz, J. \& Döring, N. (2007). Forschungsmethoden und Evaluation für Human-und Sozialwissenschaftler: Limitierte Sonderausgabe. Springer-Verlag.

Döpfner, M. (2001). Hyperaktivität und Impulsivität. In D. h. Rost (Hrsg.), Handwörterbuch pädagogische Psychologie (S. 260-267). Weinheim: Beltz PVU.

Döpfner, M., Schürman, S., \& Frölich, J. (2002). Therapieprogramm für Kinder mit hyperkinetischem und oppositionellem Problemverhalten THOP. Weinheim: Beltz.

Döpfner, M., Frölich, J., \& Lehmkuhl, G. (2013). Aufmerksamkeitsdefizit-/Hyperaktivitätsstörung (ADHS). Göttingen: Hogrefe.

DuPaul, G.J., \& Stoner, G. (2003). ADHD in the schools. New York: Guilford.

DuPaul, G.J., Weyandt, L.L., \& Janusis, G.M. (2011). ADHD in the classroom: Effective interventions strategies. Theorie into practice, 50(1), 35-42. 
Frölich, J., Döpfner, M., \& Banaschewski, T. (2014). ADHS in Schule und Unterricht: Pädagogisch-didaktische Ansätze im Rahmen des multimodalen Behandlungskonzepts. Stuttgart: Kohlhaammer.

Gawrilow, C., Guderjahn, L., \& Gold, A. (2013). ADHS: Schluss mit Chaos im Klassenzimmer. München: Reinhardt.

Gawrilow, C., Gollwitzer, P. M., \& Oettingen, G. (2011). If-then plans benefit delay of gratification performance in children with and without ADHD. Cognitive Therapy Research, 35, 442-455.

Gawrilow, C., Schmitt, K., \& Rauch, W. (2011). Kognitive Kontrolle und Selbstregulation bei Kindern mit ADHS. Kindheit und Entwicklung, 20(1), 41-48.

Heim, D., \& Nido, M. (2008). Burnout im Lehrberuf. Aarau: FHNW.

Hoberg, K. (2013). Schulratgeber ADHS. Ein Leitfaden für LehrerInnen. München: Ernst Reinhardt.

Hoza, B., Gerdes, A.C., Mrug, S., Hinshaw, S. P., Bukowski, W. M., Gold, J.A., Arnold, L. E., Abikoff, H. B., Conners, C. K., Elliott, G. R., Greenhill. L L., Hechtman, L., Jensen, P. S., Kraemer, H. C., March, J. S., Newcorn, J. H., Severe, J. B., Seanson, J. M., Vitiello, B., Wells, K. C., \& Wigal, T. (2005). Peer-assessed Outcomes in the Multimodal Treatment Study of Children with Attention-Deficit/Hyperactivity Disorder. Journal of Clinical Child and Adolescent Psychology, 34, 74-86.

Jarman, F. C. (1996). Current approaches to management of attention deficit hyperactivity disorder. The Australian Educational and Developmental Psychologist, 13, 46-55.

Kos, J. M., Richdale, A. L., \& Hay, D. A. (2006). Children with attention deficit hyperactivity disorder and their teachers: A review of the literature. International Journal of Disability, Development and Education, 53, 147-160.

Lidzba, K., Christiansen, H., \& Drechsler, R. (2013). Conners 3 (R). Conners Skalen zu Aufmerksamkeit und Verhalten - 3 (Deutschsprachige Adaptation der Conners 3rd Edition (R) (Conners $3(R)$ von C. Keith Conners). Bern: Huber.

Mandl, H. (2004). Gestaltung problemorientierter Lernumgebungen. Journal für Lehrerinnen- und Lehrerbildung, 4(3), 47-51.

Mannuzza, S., Klein, R.G., Bessler, A., Malloy P., \& Hynes, M. E. (1997). Educational and occupational outcome of hyperactive boys grown up. Journal of the American Academy of Child \& Adolescent Psychiatry, 36(9), 1222-1227.

Maslach, C., \& Jackson, S. (1981). The measurement of experienced burnout. Journal of Occupational Behavior, 2, 99-113.

Mautone, J.A., Lefler, E.K., \& Power, T.J. (2011). Promoting family and school success for children with ADHD: Strengthening relationships while building skills. Theory Into Practice, 50(1), S. 43-51.

Neuenschwander, M. P. \& Benini, S. (2017). Der FOKUS-Ansatz bei verhaltensauffälligen und unaufmerksamen Kindern konkret. Schweizerische Zeitschrift für Heilpädagogik, 23(3), 46-52..

Neuenschwander, M. P. \& Malti, T. (2009). Selektionsprozesse beim Übergang in die Sekundarstufe I und II. Zeitschrift für Erziehungswissenschaft, 12(2), 216-232.

Neuenschwander, M. P., Balmer, T., Gasser, A., Goltz, S., Hirt, U., Ryser, H., \& Wartenweiler, H. (2005). Schule und Familie - was sie zum Schulerfolg beitragen. Bern: Haupt.

Zentall, S. S. (1993). Research on the educational implications of attention deficit hyperactivity disorder. Exceptional Children, 60, 143-153. 
Open Access Dieses Kapitel wird unter der Creative Commons Namensnennung 4.0 International Lizenz (http://creativecommons.org/licenses/by/4.0/deed.de) veröffentlicht, welche die Nutzung, Vervielfältigung, Bearbeitung, Verbreitung und Wiedergabe in jeglichem Medium und Format erlaubt, sofern Sie den/die ursprünglichen Autor(en) und die Quelle ordnungsgemäß nennen, einen Link zur Creative Commons Lizenz beifügen und angeben, ob Änderungen vorgenommen wurden.

Die in diesem Kapitel enthaltenen Bilder und sonstiges Drittmaterial unterliegen ebenfalls der genannten Creative Commons Lizenz, sofern sich aus der Abbildungslegende nichts anderes ergibt. Sofern das betreffende Material nicht unter der genannten Creative Commons Lizenz steht und die betreffende Handlung nicht nach gesetzlichen Vorschriften erlaubt ist, ist für die oben aufgeführten Weiterverwendungen des Materials die Einwilligung des jeweiligen Rechteinhabers einzuholen.

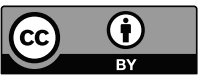




\section{Akzeptanz und Ablehnung beim Übertritt in die Sekundarstufe I}

Stephan Rösselet und Markus P. Neuenschwander

\section{Zusammenfassung}

Mit dem Übertritt in die Sekundarstufe I treten die Kinder in leistungssegregierte Schulniveaus ein und müssen ihre soziale Position in der Klasse neu aushandeln. Veränderungen der Beliebtheitsdimensionen Akzeptanz und Ablehnung beim Übertritt wurden bisher kaum untersucht. Mit Daten der Längsschnittstudie Wirkungen der Selektion WiSel wurden Akzeptanz und Ablehnung von Kindern im 5., 6. und 7. Schuljahr mit soziometrischen Verfahren erhoben. Es wurden 191 Kinder aus den Kantonen Aargau und Basel-Landschaft mit Übertritt nach dem 5. Schuljahr und 255 Kinder aus den Kantonen Bern und Luzern mit Übertritt nach dem 6. Schuljahr analysiert. Die Ergebnisse zeigen, dass hohe Akzeptanz am Ende der Primarschule mit guten Leistungen, guten Noten und hohem sozialen Selbstkonzept zusammenhängen. Diese Zusammenhänge treten aber am Anfang der Sekundarstufe I nicht auf. Die Beliebtheit ist beim Übergang in die Sekundarstufe I weniger stabil als beim Verbleib im gleichen Schultyp. Das Schulniveau und die Leistungsposition in der Klasse in Mathematik beeinflussen die Veränderung der Beliebtheit nach dem Übergang. Damit kann erstmals gezeigt werden, wie sich soziale Vergleichsprozesse auf die Veränderung der Beliebtheit beim Übertritt in die Sekundarstufe I auswirken. Schulleistungen und zugewiesenes Schulniveau auf der Sekundarstufe I beeinflussen nicht nur das Selbstkonzept, sondern auch die Beliebtheit der Schülerinnen und Schüler.

\section{Schlagworte}

Soziogramm, Beliebtheit, Transition in die Sekundarstufe I 


\section{$1 \quad$ Einleitung}

Peers sind wichtige Bezugspersonen von Jugendlichen. Sie beeinflussen ihre kognitive und soziale Entwicklung, sind für die Befriedigung des Bedürfnisses nach Kontakt, Nähe und Austausch zentral, wirken unterstützend bei der Bewältigung von Übergängen und Entwicklungsaufgaben und fördern ihre Identitätsentwicklung und Selbstpräsentation (Hannover \& Kessels, 2009). Jugendliche wollen bei Peers beliebt sein, um sich sozial zu integrieren und ihren Selbstwert zu sichern (Neuenschwander \& Hascher, 2003). Ihre Beliebtheit hängt mit schulischen Leistungen und Prüfungsversagen sowie mit ihrer psychosozialen Entwicklung zusammen (z.B. Aggressivität, Verhaltens, motorische und Aufmerksamkeitsprobleme, Kontrollüberzeugungen, Drogenmissbrauch, Delinquenz, vgl. Allen, Porter, McFarland, Marsh \& McElhaney, 2005; Ollendick, Weist, Borden \& Greene, 1992).

Die Gründe für die Unterschiede in der Beliebtheit wurden vielfach untersucht (z. B. Newcomb, Bukowski \& Pattee, 1993). Weit weniger ist darüber bekannt, ob und wie sich die Beliebtheit bei schulischen Übergängen verändert. Mit der vorliegenden Studie wird exemplarisch der Übertritt von der Primarstufe in die Sekundarstufe I untersucht. Dabei stehen Zusammenhänge der Beliebtheit mit schulischen Leistungen im Fokus, weil die Selektion leistungsbasiert ist und weil sie zu neuen, leistungshomogenen Klassen führt. Nach dem Übertritt müssen sich die Schülerinnen und Schüler mit einer veränderten Bezugsgruppe auseinandersetzen, was neue soziale Vergleichsprozesse zur Folge hat. Im vorliegenden Kapitel wird folgenden Fragen nachgegangen:

1. Wie unterscheiden sich Schülerinnen und Schüler, die in ihrer Klasse unterschiedlich beliebt sind, in ihren schulischen Leistungen und ihrem sozialen Selbstkonzept?

2. Wie stabil bleibt die Beliebtheit beim Übertritt in die Sekundarstufe I?

3. Inwiefern sagen schulische Leistungen und zugewiesenes Sekundarstufe I Niveau Veränderungen der Beliebtheit nach dem Übertritt in die Sekundarstufe I vorher?

\section{Zwei Dimensionen der Beliebtheit: Akzeptanz und Ablehnung}

Die mittels soziometrischer Verfahren gemessene Beliebtheit einer Schülerin oder eines Schülers beschreibt, wie sehr sie oder er von den Peers innerhalb des Klassenverbands gemocht wird (Hannover \& Kessels, 2009). Die Beliebtheit einer 
Schülerin oder eines Schülers stellt einen Ausdruck der Gefühle der Peers dar. Diese Gefühle hängen davon $a b$, ob und wie sehr sie die Handlungen und Eigenschaften der beurteilten Person für sie - z. B. in Bezug auf eine enge Freundschaft (Babad, 2001) - als belohnend empfinden (vgl. Thibaut \& Kelley, 1959).

Zur soziometrischen Messung von Beliebtheit nennen die Befragten eine bestimmte Anzahl Personen, die sie am liebsten und am wenigsten mögen oder mit denen sie am liebsten oder am wenigsten gern bestimmte Aktivitäten durchführen würden (liking und disliking nominations, Moreno, 1978; Moreno, 1960). Dies ermöglicht, zwei Dimensionen der Beliebtheit voneinander zu unterscheiden, die unabhängig voneinander analysiert werden können: Akzeptanz und Ablehnung (Rubin, Bukowski \& Parker, 2006). Auch ihre relativ geringe, negative Korrelation ( $r=.39$, Rost \& Czeschlik, 1994) spricht dafür, dass sie zwei unterschiedliche Dimensionen sind und nicht zwei Pole einer Dimension dasrtellen. Die getrennte Analyse der beiden Konzepte erlaubt es, die spezifischen Bedingungen von Akzeptanz und Ablehnung zu analysieren.

\subsection{Validierung von Akzeptanz und Ablehnung}

Empirische Untersuchungen zeigen, dass persönliche Eigenschaften, sozialer Hintergrund und die Beziehung zu den Eltern für die Beliebtheit relevant sind (z. B. Attili, Vermigli \& Roazzi, 2010; Dunkake, 2012; Tamm, Kasearu \& Tulviste, 2014). Bessere Schulleistungen gehen mit höherer Akzeptanz einher, während schulleistungsschwächere Schülerinnen und Schüler auf mehr Ablehnung stossen (Hatzichristou \& Hopf, 1996; Newcomb et al., 1993; Rost \& Czeschlik, 1994; Wentzel, 2005). Leistungsstarke sowie intelligente Schülerinnen und Schüler weisen Eigenschaften auf, die anderen das Erreichen von schulischen Zielen erleichtern: Sie vermitteln schulbezogene Werte und Erwartungen, dienen als positive Modelle für das Lernverhalten und geben nützlichen Rat und praktische Hilfestellungen (Hannover \& Kessels, 2009).

Für die Beliebtheit innerhalb einer Klasse sind die Leistungen und die kognitiven Fähigkeiten im internen Klassenvergleich (relative Leistungen) besonders relevant (vgl. z. B. Dunkake, 2012). Schulleistungen der Mitschülerinnen und Mitschüler sind zum einen direkt im Unterricht beobachtbar. Zum anderen sind sie indirekt über Schulnoten beobachtbar, welche als wichtige Indikatoren für die schulischen Leistungen dienen. Noten sind jedoch kein Abbild objektiver schulischer Leistungsstärke. Sie bilden Rangplätze der Schülerinnen und Schüler innerhalb der Klasse ab. Lehrpersonen greifen bei der Leistungsbeurteilung auf ein klasseninternes Bezugssystem zurück (z. B. Trautwein \& Baeriswyl, 2007), welches für klassenin- 
terne Leistungsvergleiche relevanter sein dürfte als Ergebnisse von Leistungstests. Zusammenfassend formulieren wir folgende Hypothesen:

Hypothese 1: Akzeptierte Schülerinnen und Schüler des 6. Schuljahres weisen im Vergleich mit ihren weniger akzeptierten Klassenkameradinnen und kameraden bessere Schulleistungen (relative Leistungen) und Noten auf.

Hypothese 2: Abgelehnte Schülerinnen und Schüler des 6. Schuljahres weisen im Vergleich mit ihren weniger abgelehnten Klassenkameradinnen und kameraden schlechtere Schulleistungen (relative Leistungen) und Noten auf.

Weiter spielen soziale Kompetenzen wie Kooperationsfähigkeit, Hilfsbereitschaft, Kontaktfreudigkeit, Durchsetzungsfähigkeit, Führungsqualitäten (Hannover \& Kessels, 2009; Newcomb et al., 1993; Wentzel, 2005) sowie Persönlichkeitsmerkmale (van der Linden, Scholte, Cillessen, Nijenhuis \& Segers, 2010) für Akzeptanz und Ablehnung eine wichtige Rolle. Es ist davon auszugehen, dass auch soziale Selbstkonzepte, welche ein Urteil über die eigenen sozialen Kompetenzen darstellen, mit der Beliebtheit zusammenhängen. Zudem stossen Schülerinnen und Schüler, die sich zurückziehen und stärker von Depressionen und Ängstlichkeit betroffen sind, auf Ablehnung (Cillessen, van Ijzendoorn, Van Lieshout \& Hartup, 1992; Parkhurst \& Asher, 1992). Eine positive Lebenseinstellung (Grob et al., 1991) dürfte demgegenüber mit höherer Beliebtheit einhergehen.

Hypothese 3: Akzeptierte Schülerinnen und Schüler des 6. Schuljahres schätzen sich im Vergleich mit ihren weniger akzeptierten Klassenkameradinnen und kameraden als offener, durchsetzungs- und konfliktfähiger ein und weisen eine positivere Lebenseinstellung auf.

Hypothese 4: Abgelehnte Schülerinnen und Schüler des 6. Schuljahres schätzen sich im Vergleich mit ihren weniger abgelehnten Klassenkameradinnen und kameraden als weniger offen, durchsetzungs- und konfliktfähig ein und weisen eine weniger positive Lebenseinstellung auf. 


\section{Stabilität und Veränderung der Beliebtheit beim Übertritt in die Sekundarstufe I}

Akzeptanz und Ablehnung von Schülerinnen und Schülern sind über die Zeit relativ stabil. Dies gilt sowohl für Kinder in der Vorschule (Walker, 2009) als auch für Jugendliche in der Oberstufe (Lansford, Killeya Jones, Miller \& Costanzo, 2009). Studien zeigten, dass die Stabilität mit zunehmendem Alter der Schülerinnen und Schüler sogar noch zunimmt (Jiang \& Cillessen, 2005). Weil beim Übertritt von der Primarstufe in die Sekundarstufe I neue Klassen gebildet werden, wichtige Bezugspersonen wegfallen und bestehende Hierarchien unter den Schülerinnen und Schülern verloren gehen, sind bei Schulübertritten geringere Stabilitäten zu erwarten (Jiang \& Cillessen, 2005; Pellegrini \& Bartini, 2000). Dies gilt weniger stark für die Ablehnung als für die Akzeptanz (Hardy, Bukowski \& Sippola, 2002).

Hypothese 5: Akzeptanz und Ablehnung sind beim Verbleib in der gleichen Klasse über die Zeit stabil.

Hypothese 6: Akzeptanz und Ablehnung sind bei einem Klassenwechsel in Folge des Übertritts von der Primarstufe in die Sekundarstufe I über die Zeit weniger stabil als beim Verbleib in der gleichen Klasse (vgl. Hypothese 5).

\section{Determinanten der Veränderung beim Übertritt in die Sekundarstufe I}

Beim Übertritt in die Sekundarstufe I wechseln Schülerinnen und Schüler in leistungssegregierte Schulniveaus. Dadurch werden die Leistungen innerhalb der Klassen homogener, während sie sich zwischen den Schulniveaus stärker unterscheiden. Wie stark die Klassenzusammensetzung die Schülerinnen und Schüler beeinflussen, konnte in Bezug auf das schulische Selbstkonzept mit dem Big-Fish-Little-Pond-Effekt eindrücklich belegt werden (Marsh, 1987, 2005). Ähnliche Referenzgruppeneffekte sind auch bei der Beliebtheit zu erwarten. Wir gehen davon aus, dass analog zur Beurteilung eigener schulischer Fähigkeiten auch die Beliebtheit der Klassenkameradinnen und -kameraden im Klassenverband von den Leistungen der gesamten Klasse abhängen. Wir nehmen deshalb an, dass ein hohes Leistungsniveau der Klasse nach dem Übertritt die Beliebtheit von Schülerinnen und Schülern negativ beeinflusst, weil deren individuelle Leistung im Lichte der leistungsstärkeren Referenzgruppe weniger gut erscheint. Wir erwarten auch, dass 
Schülerinnen und Schüler, die, verglichen mit ihrer neuen Klasse, gute Leistungen (relative Leistungen) erbringen, an Beliebtheit gewinnen. Während sprachliche Kenntnisse nicht nur im Fach Deutsch, sondern auch in anderen Fächern und ausserhalb der Schule gelernt, angewendet und gefördert werden, ist dies für Mathematik kaum der Fall (Angelone, Keller \& Moser, 2013). Wir erwarten deshalb positive Individualeffekte der Leistungen für das Fach Mathematik. Die Leistungen im Fach Deutsch sind aber für die Beliebtheit unerheblich.

Hypothese 7: Die Akzeptanz sinkt beim Übertritt in Klassen mit höheren Anforderungen, während sie beim Übertritt in Klassen mit niedrigeren Anforderungen zunimmt.

Hypothese 8: Die Ablehnung steigt beim Übertritt in Klassen mit höheren Anforderungen, während sie beim Übertritt in Klassen mit niedrigeren Anforderungen sinkt.

Hypothese 9: Die Akzeptanz von Schülerinnen und Schülern, die nach dem Übertritt bessere schulische Leistungen in Mathematik im Vergleich zum Klassendurchschnitt aufweisen (relative Leistungen), nimmt zu. Sie nimmt bei schlechteren relativen Mathematikleistungen ab.

Hypothese 10: Die Ablehnung von Schülerinnen und Schülern, die nach dem Übertritt bessere relative Mathematikleistungen aufweisen, nimmt ab. Sie nimmt bei schlechteren relativen Mathematikleistungen zu.

\section{$5 \quad$ Methode}

Zur Prüfung der formulierten Hypothesen wurden Daten der Schweizer Längsschnittstudie Wirkungen der Selektion ${ }^{1}$ - kurz WiSel - herangezogen, die am Zentrum Lernen und Sozialisation der Pädagogischen Hochschule FHNW durchgeführt wurde.

Schülerinnen und Schüler (5., 6. und 7. Schuljahr) aus vier Deutschschweizer Kantonen füllten in den Herbstsemestern der Schuljahre 2011/2012, 2012/2013 und 2013/2014 schriftliche Fragebögen aus und lösten Leistungstests in den Fächern Mathematik und Deutsch. Die Erhebungen in den verschiedenen Kantonen erlaubten den Vergleich unterschiedlicher Übertrittszeitpunkte in die Sekundarstufe I. Die

1 Wir danken dem Schweizerischen Nationalfonds für die Finanzierung dieser Studie (Projektnummer 100013_134594/1). Wir danken Benno Rottermann und Edith Niederbacher für die Arbeit bei der Durchführung der Studie. 
Schülerinnen und Schüler der Kantone Aargau und Basel-Landschaft traten nach dem 5. Schuljahr von der Primarschule in die Sekundarstufe I über. Diejenigen aus den Kantonen Bern und Luzern traten ein Jahr später, nach dem 6. Schuljahr, über.

\subsection{Stichprobe}

Für die vorliegenden Analysen wurden diejenigen Schülerinnen und Schüler einbezogen, für welche zu allen drei Messzeitpunkten Werte bei den Variablen zur Akzeptanz und Ablehnung vorlagen. Weil diese Variablen auf Urteilen der Mitschülerinnen und Mitschüler basieren und sich die Klassenzusammensetzungen beim Übertritt in die Sekundarstufe I veränderten, wurde auf eine Schätzung der fehlenden Werte verzichtet. Schülerinnen und Schüler in Klassen, in denen zumindest an einem der drei Messzeitpunkte weniger als zehn Schülerinnen und Schüler an den Erhebungen teilgenommen hatten, wurden von den Analysen ausgeschlossen.

Von den verbliebenen 446 Schülerinnen und Schülern gehörten 191 der Gruppe mit Übertritt nach dem 5. Schuljahr und 255 der Gruppe mit Übertritt nach dem 6. Schuljahr an. Mädchen $(231,51.8 \%)$ und Jungen $(215,48.2 \%)$ waren in etwa gleich stark vertreten. 313 (70.2\%) Schülerinnen und Schüler waren Schweizerin oder Schweizer, 92 (20.6\%) Ausländerin oder Ausländer und 41 (9.2\%) Doppelbürgerin oder Doppelbürger. Diese Verhältnisse entsprechen in etwa denjenigen der Schweizer Primarstufe im Schuljahr 2011/2012².

\subsection{Instrumente und Variablen}

Zur Beantwortung der formulierten Hypothesen werden folgende Variablen des Fragebogens und der Leistungstests benutzt ${ }^{3}$ :

2 vgl. https://www.bfs.admin.ch/bfs/de/home/statistiken/bildung-wissenschaft/personen-ausbildung/obligatorische-schule.assetdetail.246173.html

3 Eine ausführliche Beschreibung der eingesetzten Erhebungsinstrumente ist in den Dokumentationsbänden der Studie zu finden (Neuenschwander, Rösselet, Niederbacher, Rottermann \& Scheffler, 2014; Neuenschwander, Rösselet, et al., 2013; Neuenschwander, Rösselet, Scheffler \& Rottermann, 2014; Neuenschwander, Rottermann, Niederbacher, Rösselet \& Scheffler, 2014; Neuenschwander, Rottermann \& Rösselet, 2013; Neuenschwander, Rottermann, Scheffler \& Rösselet, 2014). Download: http://www.fhnw.ch/ $\mathrm{ph} /$ zls/interne-berichte/forschungsberichte/forschungsberichte-wisel. 
1. Beliebtheit (5., 6. und 7. Schuljahr): Akzeptanz und Ablehnung mit je einem Item: „Neben welchen zwei Schülerinnen oder Schülern deiner Klasse möchtest Du am liebsten/am wenigsten gerne sitzen?". Für alle Schülerinnen und Schüler wurde berechnet, wie oft sie gewählt wurden. Damit die Klassengrösse und die Anzahl der Teilnehmenden pro Klasse die Werte nicht verzerrten, wurden die Wahlhäufigkeiten daran relativiert: Der berechnete Wert entspricht einer Schätzung der Prozentzahl der Schülerinnen und Schüler, von denen die Schülerin oder der Schüler gewählt worden wäre, wenn die vollständige Klasse an den Erhebungen teilgenommen hätte. Da diese Werte nicht normalverteilt sind, wurden sie anhand der Auftretenshäufigkeit in Terzile aufgeteilt.

2. Schulnoten Deutsch und Mathematik (7. Schuljahr): Schülerangaben rückblickend auf das Ende des vorangegangenen Schuljahres (1: tiefste Note, 6: höchste Note).

3. Schulniveau Sekundarstufe I (6. / 7. Schuljahr): dreistufig (tiefe, mittlere, hohe Anforderungen).

4. Soziales Selbstkonzept (6. Schuljahr): Drei Faktoren (1) Offenheit (drei Items, z. B. „Einer guten Freundin oder einem guten Freund vertraue ich auch Dinge an, die mir unangenehm sind“, $\alpha=.77$ ), (2) Durchsetzungsvermögen (vier Items, z. B. „Andere Jugendliche hören auf mich“, $\alpha=.67$ ), (3) Konfliktfähigkeit (vier Items, z. B. „Wenn ich eine Meinungsverschiedenheit mit jemandem habe, kann ich sehr gut akzeptieren, dass die oder der Andere nicht die gleiche Meinung hat wie ich ", $\alpha=.67$ ), Antwortskala jeweils von 1 ,stimmt überhaupt nicht', bis 6 ,stimmt voll und ganz:

5. Positive Lebenseinstellung (6. Schuljahr): Acht Items (z. B. „Ich bin zufrieden mit der Art und Weise, wie sich meine Lebenspläne verwirklichen", $\alpha=.86$ ), Antwortskala von 1 ,stimmt überhaupt nicht' bis 6 ,stimmt voll und ganz'.

6. Leistungen und relative Leistungen Deutsch und Mathematik (6. und 7. Schuljahr): Auswahl von Aufgaben von Moser, Buff, Angelone \& Hollenweger (2011) mit einem Ankeritem-Design (Wiederholung einer Teilmenge der Items zu den beiden Messzeitpunkten). Auf der Grundlage der Item Response Theorie (Yen \& Fitzpatrick, 2006) wurde mittels Linking-Methoden (Haberman, 2009) die Leistungen auf einer Metrik abgebildet. Damit können tatsächliche Veränderungen der Kompetenzwerte (Weighted Likelihood Estimates, vgl. Warm, 1989) über mehrere Messzeitpunkte beschrieben werden. Die relativen Leistungen wurden berechnet, indem von der individuellen Leistung der Mittelwert der jeweiligen Klasse abgezogen wurde. 


\subsection{Durchführung der Erhebungen}

Vor den Erhebungen wurde von den Kantonen, den Schulleitungen, den Lehrpersonen und den Eltern das ausdrückliche Einverständnis zur Teilnahme an der Studie eingeholt. Die Teilnahme der Schülerinnen und Schüler war freiwillig. Instruierte Projektmitarbeitende leiteten die Schülerbefragung und die Leistungstests gemäss einer standardisierten Instruktion an. Die teilnehmenden Schülerinnen und Schüler füllten während der Unterrichtszeit im Klassenverband zuerst den Schülerfragebogen und zwei bis drei Wochen später die Leistungstests aus.

\section{$6 \quad$ Ergebnisse}

\subsection{Validierung der Beliebtheit}

Zur Validierung von Akzeptanz und Ablehnung und Testung der Hypothesen 1 bis 4 wurden mehrere einfaktorielle Varianzanalysen durchgeführt. Es wurden die Daten des 6. Schuljahres herangezogen. Damit kann die Validierung der Akzeptanz und der Ablehnung für die Situation am Ende der Primarstufe und zu Beginn der Sekundarstufe I anhand von Daten aus dem gleichen Schuljahr vorgenommen werden. In diesen Analysen sind Akzeptanz und Ablehnung jeweils die unabhängigen Variablen. Die drei Terzile (unteres, mittleres, oberes Terzil) stellen ihre Ausprägungen dar.

Die Hypothesen 1 und 2 werden mit den abhängigen Variablen Testleistungen, relative Testleistungen und Noten geprüft. Zur Prüfung von Hypothese 3 und 4 sind die drei Dimensionen Offenheit, Durchsetzungsvermögen, Konfliktfähigkeit des sozialen Selbstkonzepts und die positive Lebenseinstellung die abhängigen Variablen. 
Tab. 1 Varianzanalysen zur Validierung von Akzeptanz und Ablehnung im 6. Schuljahr

\begin{tabular}{|c|c|c|c|c|c|c|c|c|c|c|c|c|}
\hline & \multicolumn{6}{|c|}{ Akzeptanz Ende Prim. } & \multicolumn{6}{|c|}{ Akzeptanz Anfang Sek I } \\
\hline & I & II & III & $\mathrm{F}$ & & $\mathrm{df}_{1,2}$ & I & II & III & $\mathrm{F}$ & & $\mathrm{df}_{1,2}$ \\
\hline LM & 0.55 & 0.52 & 0.67 & 0.76 & & 2,247 & 0.66 & 0.54 & 0.33 & 1.92 & & 2,185 \\
\hline LD & 0.17 & 0.35 & 0.47 & 1.95 & & 2,246 & 0.57 & 0.28 & 0.03 & 3.96 & * & 2,184 \\
\hline RLM & 0.06 & 0.05 & 0.29 & 2.17 & & 2,247 & -0.03 & -0.02 & -0.19 & 1.66 & & 2,185 \\
\hline RLD & 0.03 & 0.16 & 0.34 & 2.25 & & 2,246 & 0.14 & -0.10 & -0.17 & 2.62 & & 2,184 \\
\hline NM & 4.84 & 4.87 & 5.05 & 2.87 & & 2,248 & 4.58 & 4.62 & 4.54 & 0.24 & & 2,184 \\
\hline ND & 4.86 & 4.99 & 5.19 & 7.30 & ** & 2,246 & 4.66 & 4.69 & 4.81 & 1.80 & & 2,185 \\
\hline $\mathrm{OF}$ & 4.80 & 4.71 & 4.95 & 1.49 & & 2,251 & 4.71 & 5.07 & 5.04 & 2.68 & & 2,188 \\
\hline DV & 4.32 & 4.33 & 4.61 & 4.80 & $* *$ & 2,252 & 4.28 & 4.40 & 4.53 & 1.63 & & 2,188 \\
\hline KF & 4.54 & 4.43 & 4.54 & 0.84 & & 2,252 & 4.29 & 4.47 & 4.30 & 1.25 & & 2,188 \\
\hline \multirow[t]{3}{*}{ PLE } & 4.85 & 4.93 & 5.12 & 4.50 & * & 2,252 & 4.70 & 4.85 & 4.81 & 0.70 & & 2,188 \\
\hline & \multicolumn{6}{|c|}{ Ablehnung Ende Prim. } & \multicolumn{6}{|c|}{ Ablehnung Anfang Sek I } \\
\hline & I & II & III & $\mathrm{F}$ & & $\mathrm{df}_{1,2}$ & I & II & III & $\mathrm{F}$ & & $\mathrm{df}_{1,2}$ \\
\hline LM & 0.64 & 0.62 & 0.41 & 1.49 & & 2,247 & 0.46 & 0.80 & 0.32 & 2.99 & & 2,185 \\
\hline LD & 0.44 & 0.55 & -0.10 & 7.72 & $* *$ & 2,246 & 0.23 & 0.30 & 0.42 & 0.46 & & 2,184 \\
\hline RLM & 0.22 & 0.15 & -0.09 & 2.77 & & 2,247 & -0.10 & -0.02 & -0.08 & 0.29 & & 2,185 \\
\hline RLD & 0.27 & 0.41 & -0.26 & 8.83 & $* *$ & 2,246 & -0.14 & -0.15 & 0.30 & 5.12 & $* *$ & 2,184 \\
\hline NM & 5.04 & 4.78 & 4.75 & 6.28 & $* *$ & 2,248 & 4.58 & 4.70 & 4.45 & 1.62 & & 2,184 \\
\hline ND & 5.12 & 5.03 & 4.75 & 8.89 & $* *$ & 2,246 & 4.76 & 4.69 & 4.63 & 1.30 & & 2,185 \\
\hline OF & 4.90 & 4.73 & 4.73 & 1.07 & & 2,251 & 5.10 & 4.86 & 4.65 & 3.79 & * & 2,188 \\
\hline DV & 4.50 & 4.33 & 4.33 & 1.75 & & 2,252 & 4.44 & 4.65 & 4.07 & 6.83 & $* *$ & 2,188 \\
\hline $\mathrm{KF}$ & 4.55 & 4.43 & 4.45 & 0.95 & & 2,252 & 4.45 & 4.38 & 4.10 & 3.95 & * & 2,188 \\
\hline PLE & 5.06 & 5.03 & 4.68 & 9.20 & $* *$ & 2,252 & 4.88 & 4.84 & 4.52 & 4.10 & * & 2,188 \\
\hline
\end{tabular}

Legende: Prim.: Primarstufe, Sek I: Sekundarstufe I, I: unteres Terzil, II: mittleres Terzil, III: oberes Terzil, LM: Leistungen Mathematik, LD: Leistungen Deutsch, RLM: Relative Leistungen Mathematik, RLD: Relative Leistungen Deutsch, NM: Note Mathematik, ND: Note Deutsch, OF: Offenheit, DV: Durchsetzungsvermögen, KF: Konfliktfähigkeit, PLE: Positive Lebenseinstellung, ${ }^{*}: p<.05,{ }^{* *}: p<.01$

In den Klassen, die im 6. Schuljahr am Ende der Primarstufe standen, waren folgende Unterschiede zu beobachten (Tabelle 1): Wie in Hypothese 1 formuliert, wiesen Schülerinnen und Schüler, die auf eine höhere Akzeptanz bei ihren Klassenkameradinnen und kameraden stiessen, bessere Noten im Fach Deutsch auf. Der Hypothese 2 entsprechend zeigte sich, dass Schülerinnen und Schüler, die auf eine höhere Ablehnung stiessen, schlechtere Noten in den Fächern Deutsch und Mathematik aufwiesen. Sie schnitten auch im Leistungstest Deutsch im klassen- 
internen Vergleich schlechter ab. Sie zeigten zudem schwächere Testleistungen im Fach Deutsch. Der Hypothese 3 entsprechend beschrieben sich besser akzeptierte Schülerinnen und Schüler als durchsetzungsfähiger und berichteten über eine positivere Lebenseinstellung. Der Hypothese 4 entsprechend hatten stärker abgelehnte Schülerinnen und Schüler eine weniger positive Lebenseinstellung. Auch wenn die Mittelwertsunterschiede nicht bei allen untersuchten Variablen signifikant waren, unterschieden sich die Schülerinnen und Schüler der Terzile grösstenteils in der erwarteten Richtung.

In den Klassen, die im 6. Schuljahr am Anfang der Sekundarstufe I standen, zeigte sich folgendes Bild: Es waren keine der in Hypothese 1 formulierten Unterschiede in den Leistungen zwischen den drei Terzilen der Akzeptanz zu finden. Bei den Leistungen im Fach Deutsch zeigte sich entgegen der Hypothese 1, dass Schülerinnen und Schüler, die auf eine höhere Akzeptanz stiessen, schlechter abschnitten. Auch die gemäss Hypothese 2 erwarteten Unterschiede wurden nicht gefunden. Schülerinnen und Schüler, die auf eine höhere Ablehnung bei ihren Klassenkameradinnen stiessen, wiesen im klasseninternen Vergleich bessere Leistungen im Fach Deutsch auf. Die Hypothese 3 wurde ebenfalls widerlegt. Schülerinnen und Schüler, die unterschiedlich akzeptiert wurden, unterschieden sich weder im sozialen Selbstkonzept noch in der positiven Lebenseinstellung voneinander. Entsprechend der Hypothese 4 beschrieben sich stärker abgelehnte Schülerinnen und Schüler als weniger offen, weniger konfliktfähig und wiesen eine weniger positive Lebenseinstellung auf.

Zusammenfassend können die Hypothesen 1 bis 4 für die Gruppe der Schülerinnen und Schüler, die im 6. Schuljahr am Ende der Primarstufe stehen, weitgehend bestätigt werden: Beliebte Schülerinnen und Schüler erbringen im klasseninternen Vergleich bessere Leistungen und Noten und berichten über ein höheres soziales Selbstkonzept. Für die Gruppe, die am Anfang der Sekundarstufe I steht, treffen die Hypothesen 1 bis 3 aber nicht zu. Nur Hypothese 4 wird bestätigt.

\subsection{Stabilität und Veränderung}

$\mathrm{X}^{2}$ Tests dienten der Überprüfung der Hypothesen 5 und 6 zur Stabilität und Veränderung von Akzeptanz und Ablehnung. In einer ersten Analyse wurde die beobachtete Verteilung von Abstieg, Verbleib und Aufstieg vom 5. zum 6. und vom 6. zum 7. Schuljahr einer Gleichverteilung (erwartete Werte) gegenübergestellt. Abstieg, Verbleib und Aufstieg beziehen sich auf Veränderungen in der Zugehörigkeit zu den Terzilen. Aufstieg bezeichnet einen Wechsel von einem Terzil mit weniger positiven Wahlen zu einem mit mehr Wahlen (Terzil I zu II/III oder II zu III). 
Abstieg ist der Wechsel von einem Terzil mit mehr Wahlen zu einem mit weniger Wahlen (Terzil III zu II/I oder Terzil II zu I). Stabil meint, dass die Schülerin oder der Schüler im gleichen Terzil geblieben ist.

Tab. 2 Abstieg, Verbleib und Aufstieg über die Zeit

\begin{tabular}{|c|c|c|c|c|c|c|c|}
\hline & & & $h_{b}\left(h_{e}\right)$ & & & & \\
\hline & & & Abstieg & Verbleib & Aufstieg & $\chi^{2}, d f$ & $p$ \\
\hline \multirow{4}{*}{ 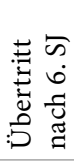 } & \multirow[t]{2}{*}{ Akzeptanz } & 5.-6. SJ (Prim) & $71(85)$ & 124(85) & $60(85)$ & $27.55,2$ & $<.01$ \\
\hline & & 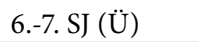 & $82(85)$ & $98(85)$ & $75(85)$ & $3.27,2$ & .20 \\
\hline & \multirow[t]{2}{*}{ Ablehnung } & 5.-6. SJ (Prim.) & $41(85)$ & $150(85)$ & $64(85)$ & $77.67,2$ & $<.01$ \\
\hline & & 6.-7. SJ (Ü) & $58(85)$ & 155(85) & $42(85)$ & $87.98,2$ & $<.01$ \\
\hline \multirow{4}{*}{ 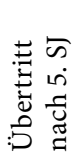 } & \multirow[t]{2}{*}{ Akzeptanz } & 5.-6. SJ (Ü.) & $55(63.7)$ & $79(63.7)$ & $57(63.7)$ & $5.57,2$ & .06 \\
\hline & & 6.-7. SJ (Sek I) & $39(63.7)$ & $115(63.7)$ & $37(63.7)$ & $62.12,2$ & $<.01$ \\
\hline & \multirow[t]{2}{*}{ Ablehnung } & 5.-6. SJ (Ü.) & $27(63.7)$ & $107(63.7)$ & $57(63.7)$ & $51.31,2$ & $<.01$ \\
\hline & & 6.-7. SJ (Sek I) & $45(63.7)$ & $120(63.7)$ & $26(63.7)$ & $77.60,2$ & $<.01$ \\
\hline
\end{tabular}

Legende: SJ: Schuljahr, Prim.: Verbleib in der Primarstufe I, Ü: Übertritt in die Sekundarstufe I, Sek I.: Verbleib in der Sekundarstufe I

Bei der Gruppe mit Übertritt nach dem 6. Schuljahr waren entsprechend Hypothese 5 vom 5. zum 6. Schuljahr beim Verbleib in der Primarstufe die Akzeptanz und die Ablehnung stabiler als bei einer Gleichverteilung (Tabelle 2). Entgegen Hypothese 6 war bei der Ablehnung die Häufigkeit von Verbleib beim Übergang vom 6. zum 7. Schuljahr mit dem Übertritt in die Sekundarstufe I häufiger als erwartet. Bei der Akzeptanz wurden der Hypothese 6 entsprechend keine Abweichungen von der Gleichverteilung gefunden.

Bei der Gruppe mit Übertritt nach dem 5. Schuljahr war vom 5. zum 6. Schuljahr mit Übertritt in die Sekundarstufe I bei der Ablehnung entgegen Hypothese 6 die Zahl der Personen im Verbleib höher als erwartet. Der Hypothese 5 entsprechend waren vom 6. zum 7. Schuljahr beim Verbleib in der Sekundarstufe I signifikante Abweichungen bei Akzeptanz und Ablehnung vorhanden. Insgesamt bestätigen diese Auswertungen die Hypothesen 5 und 6 weitgehend. Sowohl die Akzeptanz als auch die Ablehnung bleiben über die Zeit relativ stabiler. Findet der Übertritt in die Sekundartstufe I mit einem Klassenwechsel statt, ist die Stabilität der Akzeptanz geringer.

Aufgrund der berichteten hypothesenwidrigen Befunde wurden die Hypothesen 5 und 6 zusätzlich mit einer alternativen Vorgehensweise überprüft. Dazu wurden die beobachteten Häufigkeiten von Abstieg, Verbleib und Aufstieg vom 
5. zum 6. Schuljahr als Erwartungswerte $\left(h_{e}\right)$ eingesetzt und mit den beobachteten Häufigkeiten von Abstieg, Verbleib und Aufstieg vom 6. zum 7. Schuljahr $\left(h_{b}\right)$ verglichen. Damit konnte überprüft werden, ob die Verteilung der Werte vom 5. zum 6. Schuljahr mit der Verteilung vom 6. zum 7. Schuljahr übereinstimmt. Bei der Gruppe mit Übertritt nach dem 6. Schuljahr stammen die erwarteten Häufigkeiten aus der Phase ohne Übertritt und die beobachteten Häufigkeiten aus der Phase mit Übertritt. Bei der Gruppe mit Übertritt nach dem 5. Schuljahr sind die erwarteten Häufigkeiten aus der Phase mit Übertritt und die beobachten Häufigkeiten aus der Phase ohne Übertritt. Diese direkte Gegenüberstellung ist möglich, weil die Randsummen zur Berechnung der Erwartungswerte mit den Randsummen der beobachteten Werte übereinstimmen.

Tab. 3 Vergleiche der Stabilität mit und ohne Übertritt

\begin{tabular}{llrrrrr}
\hline & & $\begin{array}{c}\text { Abstieg } \\
\mathrm{h}_{\mathrm{b}}\left(\mathrm{h}_{\mathrm{e}}\right)\end{array}$ & $\begin{array}{c}\text { Verbleib } \\
\mathrm{h}_{\mathrm{b}}\left(\mathrm{h}_{\mathrm{e}}\right)\end{array}$ & $\begin{array}{c}\text { Aufstieg } \\
\mathrm{h}_{\mathrm{b}}\left(\mathrm{h}_{\mathrm{e}}\right)\end{array}$ & $\chi^{2}, d f$ & $p$ \\
\hline Übertritt nach 6. SJ & Akzeptanz & $\mathbf{8 2}(71)$ & $\mathbf{9 8}(124)$ & $\mathbf{7 5}(60)$ & $10.91,2$ & $<.01$ \\
\cline { 2 - 7 } & Ablehnung & $\mathbf{5 8}(41)$ & $\mathbf{1 5 5}(150)$ & $\mathbf{4 2}(64)$ & $14.78,2$ & $<.01$ \\
\hline Übertritt nach 5. SJ & Akzeptanz & $39(\mathbf{5 5})$ & $115(\mathbf{7 9})$ & $37(\mathbf{5 7})$ & $28.08,2$ & $<.01$ \\
\cline { 2 - 7 } & Ablehnung & $45(\mathbf{2 7})$ & $120(\mathbf{1 0 7})$ & $26(57)$ & $30.44,2$ & $<.01$ \\
\hline
\end{tabular}

Legende: fett: Häufigkeiten in einer Phase mit Übertritt in die Sekundarstufe I, SJ: Schuljahr

Dieser Vergleich zeigt gemäss Tabelle 3, dass beim Übertritt in die Sekundarstufe I Abstiege und Aufstiege bei der Akzeptanz häufiger und Verbleibe seltener auftreten, als beim Verbleib in der Primarstufe. Während z. B. bei der Gruppe mit Übertritt nach dem 6. Schuljahr vom 5. zum 6. Schuljahr 124 Schülerinnen und Schüler in ihrer Akzeptanz stabil blieben, waren es ein Jahr später mit Übertritt nur 98. Bei der Gruppe mit Übertritt nach dem 5. Schuljahr blieben vom 5. zum 6. Schuljahr während des Übertritts nur 79 stabil, ein Jahr später ohne Übertritt hingegen 115.

Bei der Ablehnung ist die Stabilität nur bei der Gruppe mit Übertritt nach dem 5. Schuljahr geringer, wenn der Übertritt stattfand (vom 5. zum 6. Schuljahr: 107), als wenn keiner stattfand (vom 6. zum 7. Schuljahr: 120). Möglicherweise verfestigt sich die Ablehnung gegenüber Schülerinnen und Schülern über die Zeit immer mehr. Dies hat zur Folge, dass bei späterem Übertritt in die Sekundarstufe I weniger Auswirkungen auf die Stabilität der Ablehnung zu finden sind.

Insgesamt belegen diese Auswertungen die hohe Stabilität der Beliebtheit (Akzeptanz und Ablehnung) über ein Schuljahr (Hypothese 5). Die vorgenommenen Auswertungen bestätigen die Hypothese 6, die besagt, dass Veränderungen der 
Beliebtheit beim Übertritt in die Sekundarstufe I häufiger auftreten als beim Verbleib in der gleichen Klasse. Dies trifft stärker für die Akzeptanz als für die Ablehnung zu.

\subsection{Determinanten der Veränderung}

Weil die Veränderungen der Beliebtheit beim Übergang in die Sekundarstufe I besonders häufig sind, wurden mit der Teilstichprobe mit Übertritt nach dem 6. Schuljahr die Hypothesen 7 bis 10 getestet. Der Einfluss des zugewiesenen Schulniveaus der Sekundarstufe I und der Leistungsposition innerhalb der neuen Klasse auf die Veränderung der Beliebtheit wurde geprüft. Dieses Vorgehen ermöglichte es, Einblicke in die Entstehung der Beliebtheit zu gewinnen.

Die Testung der Hypothesen erfolgte mittels logistischer Regressionen. Als abhängige Variable dienten die Veränderungen der Akzeptanz und der Ablehnung vom 6. zum 7. Schuljahr (Abstieg vs. Aufstieg). Die Schülerinnen und Schüler, die in dieser Zeit stabil blieben, wurden nicht in die Analyse aufgenommen. Als unabhängige Variablen wurden das dummy-kodierte Schulniveau (Grundanforderung und hohe Anforderungen) und die relativen Mathematikleistungen herangezogen.

Tab. 4 Determinanten der Veränderung beim Übertritt nach dem 6. Schuljahr (odds ratios)

\begin{tabular}{|c|c|c|c|c|c|}
\hline \multirow{3}{*}{ Tiefe Anf. } & \multicolumn{2}{|c|}{ Akzeptanz } & \multicolumn{3}{|c|}{ Ablehnung } \\
\hline & $\operatorname{Exp}(B)$ & CI $95 \%$ & \multirow{2}{*}{$\frac{\operatorname{Exp}(\mathrm{B})}{.38}$} & \multicolumn{2}{|c|}{ CI $95 \%$} \\
\hline & $2.75 *$ & 1.096 .93 & & .11 & 1.24 \\
\hline Hohe Anf. & .94 & $.45 \quad 1.98$ & $2.64 *$ & 1.01 & 6.88 \\
\hline RLM & $1.73 *$ & $1.06 \quad 2.83$ & .63 & .33 & 1.22 \\
\hline \multirow[t]{2}{*}{ Konstante } & .79 & & .63 & & \\
\hline & $\begin{array}{l}r^{2} \text { (Nagelkerke } \\
p<.01, N=154\end{array}$ & $10.58, d f=3$, & $\begin{array}{l}r^{2} \text { (Nagelkerk } \\
p<.01, N=98\end{array}$ & $x^{2}=1$ & $03, d f=3$ \\
\hline
\end{tabular}

Legende: Anf.: Anforderungen Sek I Niveau, RLM: Relative Leistungen Mathematik, ${ }^{*}: p<.05$

Den Hypothesen 7 und 8 entsprechend beeinflusste das Schulniveau auf der Sekundarstufe I die Veränderung der Akzeptanz und der Ablehnung. Die Wahrscheinlichkeit eines Aufstiegs in der Akzeptanz war im Schulniveau mit Grundanforderungen 2.75-mal höher als im Schulniveau mit mittleren Anforderungen. Die Wahrscheinlichkeit, nach dem Übertritt stärker abgelehnt zu werden, war 
demgegenüber im Schulniveau mit hohen Anforderungen 2.64-mal höher als im Schulniveau mit mittleren Anforderungen.

Entsprechend der Hypothese 9 wiesen die Mathematikleistungen im Klassenvergleich einen positiven Effekt auf die Veränderung der Akzeptanz auf. Ihr Effekt auf die Veränderung der Ablehnung war entgegen der Hypothese 10 nicht signifikant.

\section{Diskussion}

Der vorliegende Beitrag befasst sich mit der Beliebtheit von Schülerinnen und Schülern anhand der zwei Dimensionen Akzeptanz und Ablehnung. Es wurde erstmals gezeigt, welche Konsequenzen der Übertritt in die leistungssegregierte Sekundarstufe I auf die Beliebtheit hat. Dabei wurde auf den Einfluss von individuellen und Klassenleistungen fokussiert. Die durchgeführten Analysen belegen, dass der Übertritt in die Sekundarstufe I mit Veränderungen der Beliebtheit einhergehen, die mit dem Big-Fish-Little-Pond-Effekt (Marsh, 1987, 2005) vergleichbar sind. Die Beliebtheit der Kinder verändert sich beim Schulübertritt abhängig vom zugewiesenen Schulniveau und der Leistungsposition in der Klasse in Mathematik.

Die Befunde zur Validierung von Akzeptanz und Ablehnung am Ende der Primarstufe bestätigen bekannte Unterschiede der Beliebtheit von Schülerinnen und Schülern, die sich in ihren Leistungen voneinander unterscheiden. Es sind insbesondere Schülerinnen und Schüler mit geringeren Schulleistungen, welche abgelehnt werden. Am Anfang der Sekundarstufe I sind solche leistungsbezogenen Differenzen hingegen noch nicht zu finden. Schülerinnen und Schüler mit geringeren Leistungen sind dann eher beliebter und solche mit besseren Leistungen werden eher abgelehnt. Wir vermuten, dass unmittelbar nach dem Übertritt in die gegliederte Sekundarstufe I schulische Leistungen durch veränderte Vergleichsprozesse in einem neuen Licht erscheinen.

Auch die Analysen zur Stabilität von Akzeptanz und Ablehnung legen nahe, dass die Validierung in der Situation zu Beginn der Sekundarstufe I nicht gelingt, weil nach dem Übertritt soziale Positionen in den Klassen neu ausgehandelt werden. Die Beliebtheit von Schülerinnen und Schülern verändert sich beim Übertritt, der mit einem Wechsel der Bezugsgruppe einhergeht, stärker als beim Verbleib in der gleichen Klasse.

Die durchgeführten logistischen Regressionen lassen vermuten, dass zumindest ein Teil dieser Veränderungen auf Ergebnisse von Leistungsvergleichen innerhalb der neu zusammengesetzten Klassen zurückzuführen ist. Weil individuelle Leistungen je nach Vergleichsgruppe unterschiedlich beurteilt werden, sind Schülerinnen 
und Schüler, welche in eine Klasse mit höheren Anforderungen wechseln, in der neuen Klasse weniger attraktiv als vor dem Übertritt. Sie verlieren an Beliebtheit. Andererseits profitieren Schülerinnen und Schüler, die im Klassenvergleich bessere Leistungen zeigen, vom Übertritt. Sie gewinnen an Beliebtheit.

Weiter konnte gezeigt werden, dass sich beliebtere Schülerinnen und Schüler am Ende der Primarstufe als durchsetzungsfähiger einschätzen und über eine positivere Lebenseinstellung verfügen. Zu Beginn der Sekundarstufe I korrespondieren ein geringeres Durchsetzungsvermögen, weniger Offenheit und Konfliktfähigkeit sowie eine weniger positive Lebenseinstellung mit einer stärkeren Ablehnung. Möglicherweise stellt die Situation nach dem Übertritt eine sensible Phase für Schülerinnen und Schüler dar, in welcher soziale Selbstkonzepte besonders stark mit Ablehnung durch andere interagieren.

Aufgrund der hohen Ausfallquoten beim Schulübergang und der fehlenden Beachtung der geschachtelten Mehrebenenstruktur der Stichprobe sollten die Ergebnisse vor einer weitergehenden Interpretation repliziert werden. Weil die Veränderung der Beliebtheit bei Schulübertritt bisher kaum untersucht worden ist, erachten wir bereits die deskriptiven Vergleiche am Ende der Primarstufe und zu Beginn der Sekundarstufe I als interessant. Zusätzlich liefern die weiteren Ergebnisse Erklärungen, wie sich die Beliebtheit in Abhängigkeit vom zugewiesenen Schulniveau und der Leistungsposition in Mathematik verändert. Schulleistungen stellen aber nur eines von vielen Merkmalen dar, welches die Beliebtheit beeinflusst. Es sind Untersuchungen wünschenswert, die den Einfluss weiterer Merkmale von Schülerinnen und Schülern, Schulklassen und Lehrpersonen auf die Veränderung der Beliebtheit beim Schulübertritt einbeziehen. Ebenso wichtig ist die Umsetzung langfristig angelegter Studien, welche die Folgen der Veränderung der Beliebtheit beim Schulübertritt auf die psychosoziale Entwicklung der Jugendlichen untersuchen. Damit kann unser Verständnis der Prozesse, die beim Schulübertritt ablaufen, vertieft werden.

\section{Literatur}

Allen, J. P., Porter, M. R., McFarland, F. C., Marsh, P., \& McElhaney, K. B. (2005). The two faces of adolescents' success with peers: Adolescent popularity, social adaptation, and deviant behavior. Child Development, 76(3), 747-760.

Angelone, D., Keller, F., \& Moser, U. (2013). Entwicklung schulischer Leistungen während der obligatorischen Schulzeit. Bericht zur vierten Zürcher Lernstandserhebung zuhanden der Bildungsdirektion des Kantons Zürich. Zürich: Bildungsdirektion Kanton Zürich. 
Attili, G., Vermigli, P., \& Roazzi, A. (2010). Children's social competence, peer status, and the quality of mother-child and father-child relationships. European Psychologist, 15(1), 23-33. doi: 10.1027/1016-9040/a000002

Babad, E. (2001). On the conception and measurement of popularity: more facts and some straight conclusions. Social Psychology of Education, 5, 3-30.

Cillessen, A. H. N., van Ijzendoorn, H. W., Van Lieshout, C. F. M., \& Hartup, W. W. (1992). Heterogeneity among peer-rejected boys: Subtypes and stabilities. Child Development, 63(4), 893-905.

Dunkake, I. (2012). Soziale Netzwerke von Schülern: Beispiele angewandter Netzwerkanalysen. In M. Hennig \& C. Stegbauer (Hrsg.), Die Integration von Theorie und Methode in der Netzwerkforschung. Wiesbaden: Springer VS.

Grob, A., Lüthi, R., Kaiser, F. G., Flammer, A., Mackinnon, A., \& Wearing, A. J. (1991). Berner Fragebogen zum Wohlbefinden Jugendlicher (BFW). Diagnostica, 37, (66-75).

Haberman, S. J. (2009). Linking parameter estimates derived from an item response model through separate calibrations. ETS Research Report Series. Princeton, NJ: ETS.

Hannover, B., \& Kessels, U. (2009). Gleichaltrige. In E. Wild \& J. Möller (Hrsg.), Pädagogische Psychologie (S. 283-304). Berlin, Heidelberg: Springer.

Hardy, C. L., Bukowski, W. M., \& Sippola, L. K. (2002). Stability and change in peer relationships during the transition to middle-level school. Journal of Early Adolescence, 22(2), 117-142.

Hatzichristou, C., \& Hopf, D. (1996). A multiperspective comparison of peer sociometric status groups in childhood and adolescence. Child Development, 67(3), 1085-1102. doi: $10.2307 / 1131881$

Jiang, X. L., \& Cillessen, A. H. N. (2005). Stability of continuous measures of sociometric status: a meta-analysis. Developmental Review, 25(1), 1-25. doi: 10.1016/j.dr.2004.08.008

Lansford, J. E., Killeya-Jones, L. A., Miller, S., \& Costanzo, P. R. (2009). Early adolescents' social standing in peer groups: behavioral correlates of stability and change. Journal of Youth and Adolescence, 38(8), 1084-1095. doi: 10.1007/s10964-009-9410-3

Marsh, H. W. (1987). The Big-Fish-Little-Pond effect on academic self-concept. Journal of Educational Psychology, 79(3), 280-295.

Marsh, H. W. (2005). Big-Fish-Little-Pond Effect on academic self-concept. Zeitschrift für Pädagogische Psychologie, 19(3), 119-129. doi: 10.1024/1010-0652.19.3.119

Moreno, J. L. (1978). Who Shall Survive? Foundations of sociometry, group psychotherapy and sociodrama (3 ed.). Beacon, New York: Beacon House Inc.

Moreno, J. L. (Hrsg.). (1960). The sociometry reader. Glencoe, IL: The Free Press.

Moser, U., Buff, A., Angelone, D., \& Hollenweger, J. (2011). Nach sechs Jahren Primarschule. Deutsch, Mathematik und motivational-emotionales Befinden am Ende der 6. Klasse. Zürich: Bildungsdirektion Kanton Zürich.

Neuenschwander, M. P. \& Hascher, T. (2003). Zufriedenheit von Schülerinnen und Schülern und ihre soziale Integration. Psychologie in Erziehung und Unterricht, 50, 270-280.

Neuenschwander, M. P., Rösselet, S., Niederbacher, E., Rottermann, B., \& Scheffler, L. (2014). Wirkungen der Selektion WiSel. Dokumentation des Fragebogens für Schülerinnen und Schüler der Welle 3 2013/2014 (Forschungsbericht). Solothurn: Pädagogische Hochschule FHNW, Zentrum Lernen und Sozialisation.

Neuenschwander, M. P., Rösselet, S., Rottermann, B., Singer, A., Scheffler, L., \& Ziehli, M. (2013). Wirkungen der Selektion WiSel. Dokumentation des Fragebogens für Schülerinnen 
und Schüler der Welle 1 2011/2012 (Forschungsbericht). Solothurn: Pädagogische Hochschule FHNW, Zentrum Lernen und Sozialisation.

Neuenschwander, M. P., Rösselet, S., Scheffler, L., \& Rottermann, B. (2014). Wirkungen der Selektion WiSel. Dokumentation des Fragebogens für Schülerinnen und Schüler der Welle 22012 (Forschungsbericht). Solothurn: Pädagogische Hochschule FHNW, Zentrum Lernen und Sozialisation.

Neuenschwander, M. P., Rottermann, B., Niederbacher, E., Rösselet, S., \& Scheffler, L. (2014). Wirkungen der Selektion WiSel. Dokumentation der Leistungstests Mathematik und Deutsch der Welle 3 2013/2014 (Forschungsbericht). Solothurn: Pädagogische Hochschule FHNW, Zentrum Lernen und Sozialisation.

Neuenschwander, M. P., Rottermann, B., \& Rösselet, S. (2013). Wirkungen der Selektion WiSel. Dokumentation der Leistungstests Mathematik und Deutsch der Welle 1 2011/2012 (Forschungsbericht). Solothurn: Pädagogische Hochschule FHNW, Zentrum Lernen und Sozialisation.

Neuenschwander, M. P., Rottermann, B., Scheffler, L., \& Rösselet, S. (2014). Wirkungen der Selektion WiSel. Dokumentation der Leistungstests Deutsch und Mathematik der Welle 22012 (Forschungsbericht). Solothurn: Pädagogische Hochschule FHNW, Zentrum Lernen und Sozialisation.

Newcomb, A. F., Bukowski, W. M., \& Pattee, L. (1993). Children's peer relations: A meta-analytic review of popular, rejected, neglected, controversial, and average sociometric status. Psychological Bulletin, 113(1), 99-128. doi: 10.1037/0033-2909.113.1.99

Ollendick, T. H., Weist, M. D., Borden, M. C., \& Greene, R. W. (1992). Sociometric status and academic, behavioral, and psychological adjustment: A five-year longitudinal study. Journal of Consultingand Clinical Psychology, 60(1), 80-87.

Parkhurst, J. T., \& Asher, S. R. (1992). Peer rejection in middle school: subgroup differences in behavior, loneliness, and interpersonal concerns. Developmental Psychology, 28(2), 231-241.

Pellegrini, A. D., \& Bartini, M. (2000). A Longitudinal study of bullying, victimization, and peer affiliation during the transition from Primary school to middle school. American Educational Research Journal, 37(3), 699-725.

Rost, D. H., \& Czeschlik, T. (1994). Beliebt und intelligent? Abgelehnt und Dumm? Eine soziometrische Studie an 6500 Grundschulkindern. Zeitschrift für Sozialpsychologie, 25, 170-176.

Rubin, K. H., Bukowski, W. M., \& Parker, J. G. (2006). Peer interactions, relationships, and groups. In N. Eisenberg (Ed.), Handbook of child psychology: Social, emotional, and personality development (pp. 571-645). New York: Wiley.

Tamm, A., Kasearu, K., \& Tulviste, T. (2014). The role of family in adolescents' peer acceptance. Personal Relationships, 21(3), 420-432. doi: 10.1111/pere.12041

Thibaut, J. W. \& Kelley, H. H. (1959). The social psychology of groups. New York: Wiley.

Trautwein, U., \& Baeriswyl, F. (2007). Wenn leistungsstarke Klassenkameraden ein Nachteil sind. Zeitschrift für Pädagogische Psychologie, 21(2), 119-133. doi: 10.1024/10100652.21.2.119

van der Linden, D., Scholte, R. H. J., Cillessen, A. H. N., Nijenhuis, J. T., \& Segers, E. (2010). Classroom ratings of likeability and popularity are related to the Big Five and the general factor of personality. Journal of Research in Personality, 44(5), 669-672. doi: 10.1016/j. jrp.2010.08.007 
Walker, S. (2009). Sociometric stability and the behavioral correlates of peer acceptance in early childhood. The Journal of Genetic Psychology, 170(4), 339-358. doi: 10.1080/00221320903218364

Warm, T. A. (1989). Weighted likelihood estimation of ability in item response theory. Psychometrika, 54(3), 427-450.

Wentzel, K. R. (2005). Peer relationships, motivation, and academic performance at school. In A. J. Elliot \& C. S. Dweck (Eds.), Handbook of competence and motivation (pp. 279296). New York: Guilford Publications.

Yen, W. M., \& Fitzpatrick, A. R. (2006). Item response theory. In R. L. Brennan (Ed.), Educational Measurement (pp. 111-154). Westport: Praeger Publishers.

Open Access Dieses Kapitel wird unter der Creative Commons Namensnennung 4.0 International Lizenz (http://creativecommons.org/licenses/by/4.0/deed.de) veröffentlicht, welche die Nutzung, Vervielfältigung, Bearbeitung, Verbreitung und Wiedergabe in jeglichem Medium und Format erlaubt, sofern Sie den/die ursprünglichen Autor(en) und die Quelle ordnungsgemäß nennen, einen Link zur Creative Commons Lizenz beifügen und angeben, ob Änderungen vorgenommen wurden.

Die in diesem Kapitel enthaltenen Bilder und sonstiges Drittmaterial unterliegen ebenfalls der genannten Creative Commons Lizenz, sofern sich aus der Abbildungslegende nichts anderes ergibt. Sofern das betreffende Material nicht unter der genannten Creative Commons Lizenz steht und die betreffende Handlung nicht nach gesetzlichen Vorschriften erlaubt ist, ist für die oben aufgeführten Weiterverwendungen des Materials die Einwilligung des jeweiligen Rechteinhabers einzuholen.

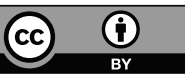




\section{Schulniveau- und Leistungserwartungen von Lehrpersonen und Leistungsentwicklung beim Übergang in die Sekundarstufe I}

Markus P. Neuenschwander \& Edith Niederbacher

\section{Zusammenfassung}

Lehrpersonenerwartungen beeinflussen Leistungen. Diese Erwartungen können sich auf die Schülerleistungen oder das Schulniveau beziehen, können implizit oder explizit sein. Erwartungseffekte auf die Leistungsentwicklung vom 5. ins 6. sowie vom 5. ins 7. Schuljahr wurden anhand einer Schweizer Stichprobe vergleichend für Schülerinnen und Schüler mit Übertritt in die Sekundarstufe I nach dem 5. Schuljahr $(N=499)$ und mit Übertritt in die Sekundarstufe I nach dem 6. Schuljahr $(N=374)$ regressionsanalytisch überprüft. Die Ergebnisse zeigen, dass implizite, nicht kommunizierte Schulniveauerwartungen von Lehrpersonen durch explizite leistungsbezogene Lehrpersonenerwartungen vermittelt werden. Kommunizierte Schulniveauerwartungen von Primarschullehrpersonen prognostizieren die differenzielle Leistungsentwicklung in Mathematik und Deutsch in den verschiedenen Schulniveaus sehr gut. Verschiedene Wirkungen von Lehrpersonenerwartungen werden diskutiert.

\section{Schlagworte}

Erwartungen, Leistungen, Schulübergang, Pygmalion 


\section{$1 \quad$ Einleitung und Fragestellung}

Immer wieder zeigten Studien und Metaanalysen, dass Lehrpersonenerwartungen die Leistungen von Schülerinnen und Schülern beeinflussen (Rosenthal \& Jacobson, 1974; Dusek \& Joseph, 1983). Lehrpersonenerwartungen tragen zur Erklärung bei, wie Unterricht die Leistungsentwicklung der Kinder beeinflusst (Eccles \& Roeser, 2011). Allerdings richten Lehrpersonen bei gleichen Leistungen der Schülerinnen und Schüler geringere Erwartungen an Kinder aus tiefen sozialen Schichten, mit Migrationshintergrund oder mit wenig familiärer Unterstützung (Tenenbaum \& Ruck, 2007, Jussim, Harber, Crawford, Cain \& Cohen, 2005; Neuenschwander, 2014). Bisher wurden aber die Wirkungen von leistungsbezogenen Lehrpersonenerwartungen nicht mit impliziten, nicht kommunizierten oder expliziten, kommunizierten Schulniveauerwartungen verglichen. Diese Unterscheidung ist hilfreich, weil sich Lehrpersonenerwartungen zwischen Schulfächern (Eccles, Wigfield \& Schiefele, 1998), der Beobachtungseinheit Individuum und Klasse (Friedrich, Flunger, Nagengast, Jonkmann \& Trautwein, 2015) sowie zwischen den Leistungen und Bildungsverläufen (Englund, Egeland, Collins, 2008) unterscheiden. Wie wirken sich verschiedene Arten von Lehrpersonenerwartungen und ihre Wechselwirkungen auf die Leistungsentwicklung von Schülerinnen und Schülern in Mathematik und Deutsch beim Übergang in die Sekundarstufe I aus? Sind Erwartungseffekte auch nach einem Schulübertritt nachweisbar?

Bereits Rosenthal (1968) berichtete von einer Intelligenzsteigerung bei Versuchspersonen durch hohe Erwartungen der Versuchsleitung, was mit Verweis auf die griechische Sage als Pygmalion-Effekt bezeichnet wurde. Im Anschluss daran berichteten Rosenthal \& Jacobs (1974) Effekte von Lehrpersonenerwartungen auf die Intelligenz von Schülerinnen und Schülern. Auch wenn diese Studien teilweise wegen methodischer Mängel kritisiert wurden, wurde im Anschluss daran in vielen Studien und Metaanalysen ein Einfluss von Lehrpersonenerwartungen auf die Schülerleistungen gezeigt (z. B. Dusek \& Joseph, 1983, Überblick in Ludwig, 1998 und Jussim \& Harber, 2005), wenngleich die Effekte oft nur klein bis mittelmässig waren (Jussim, Robustelli \& Cain, 2009). Obwohl Lehrpersonenerwartungen hinsichtlich der Schülerleistungen meist akkurat sind, bilden sie auch selbsterfüllende Prophezeiungen und schaffen somit eine soziale Realität (Jussim, Eccles, Madon, 1996). Lehrpersonenerwartungen tragen dazu bei, dass sich Kinder entsprechend dieser Erwartungen verändern.

Die Lehrpersonenerwartungen an die Schülerinnen und Schüler haben verschiedene Inhalte. Wir unterscheiden leistungsbezogene Erwartungen und Schulniveauerwartungen. Leistungsbezogene Lehrpersonenerwartungen beschreiben die Einschätzung der Schülerleistungen durch die Lehrperson im Klassenvergleich. 
Schulniveauerwartungen unterscheiden wir nach der Form in implizite, nicht kommunizierte und explizite, kommunizierte Schulniveauerwartungen. Schulniveauerwartungen von Lehrpersonen beziehen sich auf eines von drei Schulniveaus der Sekundarstufe I, welchem ein Kind voraussichtlich zugewiesen wird. Sie resultieren aus einer Einschätzung der Schulkarriere des Kindes durch die Lehrperson in der Primarstufe (Ditton \& Krüsken, 2009).

\subsection{Nicht kommunizierte Schulniveauerwartungen und Leistungserwartungen}

Lehrpersonen schätzen das zukünftige Schulniveau der Sekundarstufe I der Schülerinnen und Schüler ihrer Klasse bereits zwei Jahre vor dem Übertritt in die Sekundarstufe I, d.h. vor Beginn der Beurteilungsphase, recht genau ein (Ditton \& Krüsken, 2009). Ihre Einschätzungen sind hierbei zuverlässiger als diejenigen der Eltern (Neuenschwander, Rottermann, Rösselet, Niederbacher, 2015). Diese Schulniveauerwartung basiert auf einer impliziten, d.h. grob eingeschätzten Beurteilung der schulischen Leistungen und der Schülerintelligenz, aber auch auf der familiären Unterstützung und der Erwartungen von Eltern an ihr Kind (Neuenschwander et al., 2015; Glock, Kneer \& Kovacs, 2013). Sie wird nicht explizit kommuniziert. Die nicht kommunizierte Schulniveauerwartung wirkt sich auf die Leistungsentwicklung aus, weil sie mit entsprechenden impliziten Leistungserwartungen an die Kinder verbunden ist bzw. durch diese im Sinn einer Mediation erklärt wird. Wenn Lehrpersonen die Zuweisung in ein Schulniveau mit hohen Anforderungen erwarten, erwarten sie hohe Leistungen, mit denen die hohen Schulniveauanforderungen erfüllt werden können. Konkret: Wenn Lehrpersonen im 5. Schuljahr die Zuweisung in ein Schulniveau mit hohen Anforderungen im 7. Schuljahr erwarten, müssten die Leistungen der Schülerinnen und Schüler vom 5. zum 6. Schuljahr stärker zunehmen als bei einer Erwartung in ein Schulniveau mit tiefen Anforderungen.

Nach Harris \& Rosenthal (1985) beeinflussen Lehrpersonenerwartungen die Schülerleistungen, weil Lehrpersonen bei hohen Erwartungen den Schülerinnen und Schülern anspruchsvollere Aufgaben zuweisen, motivationsförderliche Rückmeldungen geben, eine positivere Beziehung zu ihnen haben und ihnen mehr Interaktionsgelegenheiten bieten. Damit übereinstimmend konnten Rubie-Davis, Peterson, Sibley \& Rosenthal (2015) experimentell Lehrpersonenerwartungen verändern und zeigen, dass sich die Erwartungen über die Interaktionen mit den Kindern auf die Leistungen auswirken. In Weiterführung dazu wird angenommen, dass Kinder die Erwartungen ihrer Lehrpersonen verinnerlichen, sich so 
verhalten, dass sie diesen Erwartungen entsprechen und die Wirkungen auch bei einem Lehrpersonenwechsel vorhanden bleiben. Entsprechend fanden Studien von Brophy (1985) Wirkungen von Lehrpersonenerwartungen auf die Schülerleistungen auch bei einem Lehrpersonenwechsel. Vor diesem Hintergrund wurden folgende Hypothesen formuliert:

Hypothese 1 Nicht kommunizierte Schulniveauerwartungen von Primarschullehrpersonen beeinflussen die Schülerleistungen in Mathematik und Deutsch (1a) bereits am Ende der Primarschule und (1b) nach dem Eintritt in die Sekundarstufe I.

Hypothese 2 Der Einfluss der nicht kommunizierten Schulniveauerwartungen von Primarschullehrpersonen auf Schülerleistungen in Mathematik und Deutsch wird sowohl (2a) am Ende der Primarschule als auch (2b) nach dem Eintritt in die Sekundarstufe I durch leistungsbezogene Lehrpersonenerwartungen vermittelt (Mediation).

\subsection{Kommunizierte Schulniveauerwartungen}

Das von Lehrpersonen schon vor dem Beginn des Selektionsverfahrens für die Sekundarstufe I erwartete Schulniveau wird nur teilweise reflektiert. Es wird den Schülerinnen und Schülern sowie den Eltern in der Regel nicht explizit kommuniziert. Im Unterschied dazu werden die Schulniveauerwartungen von Lehrpersonen am Ende einer Beobachtungs- und Beurteilungsphase mit den Eltern im Rahmen von sogenannten Übertrittsgesprächen gemäss Vorgaben kantonaler Gesetze diskutiert. Je nach Kanton legen Lehrpersonen ein Schulniveau fest, in das ein Kind etwa ein halbes Jahr später übertreten wird und kommunizieren dies dem Kind und dessen Eltern. In anderen Kantonen legen Lehrpersonen und Eltern im Konsensverfahren ein Schulniveau fest. Dieser vorläufige Schulniveauentscheid kann aufgrund von Prüfungsergebnissen oder Rekursen korrigiert werden, ist aber in der Regel endgültig. Der vorläufige Entscheid äussert sich in einer kommunizierten Schulniveauerwartung der Lehrperson, weil sie den Eltern und ihren Kindern, aber auch den anderen Kindern der Klasse explizit bekannt ist. Entsprechend sollte die kommunizierte Schulniveauerwartung von Lehrpersonen mit der Schulniveauerwartung von Eltern deutlich stärker korrespondieren als die nicht kommunizierte Schulniveauerwartung. Daraus resultiert zur Validierung der Interpretation der Schulniveauerwartung die dritte Hypothese: 
Hypothese 3 Kommunizierte Schulniveauerwartungen von Lehrpersonen stimmen mit den Schulniveauerwartungen von Eltern stärker überein als nicht kommunizierte Schulniveauerwartungen (Validierungshypothese).

Analog zu den nicht kommunizierten Schulniveauerwartungen führen auch kommunizierte hohe Schulniveauerwartungen von Lehrpersonen zu einer Leistungszunahme der Schülerinnen und Schüler. Wir nehmen an, dass während die nicht kommunizierten Schulniveauerwartungen zu einer beiläufigen, nicht reflektierten Förderung von Kindern führen, die kommunizierten Schulniveauerwartungen mit einer geplanten inneren Differenzierung im Unterricht einhergehen. Analog zur differenziellen Förderung in unterschiedlich anspruchsvollen Schulniveaus mit entsprechend unterschiedlich starken Leistungszuwächsen (vgl. Schereneffekte nach Baumert, Stanat \& Waterman, 2006) dürfte die erwartete Zuweisung in ein Schulniveau mit hohen Anforderungen zu einer angereicherten Förderung der Kinder, ergänzt mit einem entsprechenden Fähigkeitsselbstkonzept und Leistungsmotivation führen. Diese angereicherte Förderung setzt sich nach Eintritt in ein Schulniveau mit hohen Anforderungen fort, verstärkt durch entsprechende Kompositionseffekte (Baumert et al., 2006). Demnach wirken sich kommunizierte Schulniveauerwartungen nicht aufgrund von Leistungserwartungen der Lehrpersonen auf die Leistungen aus, sondern aufgrund der geplanten, an das Kind angepassten, aber ausgeprägten Förderung. Folgende Hypothesen werden daher überprüft:

Hypothese 4 Kommunizierte Schulniveauerwartungen von Primarschullehrpersonen beeinflussen die Leistungsentwicklung in Mathematik und Deutsch (4a) beim Übergang in die Sekundarstufe I und (4b) nach dem Eintritt in die Sekundarstufe I.

Hypothese 5 Der Einfluss kommunizierter Schulniveauerwartungen von Lehrpersonen auf Schülerleistungen in Mathematik und Deutsch wird (5a) weder beim Verbleib in der Primarschule (6. Schuljahr) noch (5b) beim Übergang in die Sekundarstufe I (7. Schuljahr) durch leistungsbezogene Lehrpersonenerwartungen vermittelt (fehlende Mediation).

\subsection{Fachunspezifität von Erwartungseffekten}

Zur Fachspezifität von Erwartungseffekten gibt es kaum Forschungsliteratur. Die Prozesse, die der Wirkung von Erwartungseffekten zu Grunde liegen, sind unab- 
hängig vom Fachinhalt (Eccles et al., 1983). Entsprechend wurden Erwartungseffekte auf die Leistungen in verschiedenen Schulfächern gezeigt (Mathematik: z. B. Friedrich, et al., 2015, Naturwissenschaften: z. B. Trouilloud, Sarrazin, Martinek, Guillet, 2002; Lesen: McKown \& Weinstein, 2008). Die Wirkungen der leistungsbezogenen Erwartungen sowie der nicht kommunizierten und kommunizierten Schulniveauerwartungen von Lehrpersonen werden daher in Bezug auf zwei Schulfächer, Mathematik und Deutsch, geprüft.

\section{$2 \quad$ Methode}

\subsection{Stichprobe}

Die Hypothesen wurden mit Daten aus der Schweizer Studie „Wirkungen der Selektion WiSel“ überprüft. Das Design der Studie basiert auf drei Messzeitpunkten (5., 6. und 7. Schuljahr), wobei sich die beiden Vergleichsgruppen im Zeitpunkt des Übertritts in die Sekundarstufe I unterschieden: In den Kantonen Aargau und Basel-Landschaft traten die Schülerinnen und Schüler nach dem 5. Schuljahr in die Sekundarstufe I über, in den Kantonen Bern und Luzern nach dem 6. Schuljahr. Zum dritten Messzeitpunkt befand sich die Gruppe mit frühem Übertritt bereits ein Jahr in der leistungsdifferenzierten Sekundarstufe I (mit drei Schulniveaus: Grundanforderungen, erweiterte Anforderungen, hohe Anforderungen), während die Gruppe mit spätem Übertritt diesen gerade erst vollzogen hatte. Aus den vier Kantonen wurden mit dem Einverständnis der kantonalen Verwaltungen zufällig Schulen ausgewählt und deren Schulleitungen und Klassenlehrpersonen um das Einverständnis zur Teilnahme an der Studie gebeten. Die Eltern meldeten ihre Kinder schriftlich zur Teilnahme an der Studie an. Die Stichprobe ist für die involvierten Kantone repräsentativ.

Bei der Gruppe mit frühem Übertritt unterschied sich die Zusammensetzung der Längsschnittstichprobe (Teilnahme im 5. und 6. Schuljahr) nicht systematisch von der restlichen Stichprobe (Leistungen Mathematik t1: $t=1.07, d f=652, n s, d=0.09$, Leistungen Deutsch t1: $t=0.83, d f=646, n s, d=0.07$, Leistungsbezogene Lehrpersonenerwartungen $\mathrm{t} 1$ : $t=0.18, d f=635, n s, d=0.015$, Schulniveauerwartungen von Lehrpersonen t1: $\left.C h i^{2}=1.354, d f=2, n s\right)$. Bei der Gruppe mit spätem Übertritt gab es zwischen Längsschnitt- und restlicher Stichprobe gewisse signifikante Unterschiede, diese sind jedoch aufgrund der geringen Effektstärken vernachlässigbar (Leistungen Mathematik t1: $t=2.99, d f=838, p<0.05, d=0.26$, Leistungen Deutsch t1: $t=4.46, d f=837, p<0.05, d=0.40$, Leistungsbezogene Lehrpersonenerwartun- 
gen: $t=3.52, d f=709, p<0.05, d=0.34$, Schulniveauerwartungen von Lehrpersonen: $\left.\mathrm{Chi}^{2}=7.63, d f=2, n s\right)$. Die Befunde zu den Rücklaufverzerrungen vom 6. zum 7 . Schuljahr waren ähnlich.

Da keine bzw. vernachlässigbare Rücklaufverzerrungen zwischen der Querschnittstichprobe zu t1 und der Längsschnittstichprobe vorlagen, wurden fehlende Werte imputiert. Imputiert wurde für die Variablen Leistungstestwerte in Mathematik und Deutsch zu allen drei Messzeitpunkten, IQ, Schulniveauerwartung sowie leistungsbezogene Erwartung der Lehrperson. Kontrolliert wurden die Variablen familiärer sozioökonomischer Status (ISEI), Geschlecht der Schülerinnen und Schüler und die Schulniveauzugehörigkeit in der 7. Klasse.

Die Längsschnittstichprobe mit imputierten Daten setzt sich wie folgt zusammen: Die Stichprobe der Gruppe mit frühem Übertritt bestand zum ersten Messzeitpunkt aus insgesamt 499 Kindern aus 74 Klassen in 25 Schulen (Anteil Mädchen $=51.3 \%$, Durchschnittsalter 10.9 Jahre). In der Gruppe mit spätem Übertritt befanden sich 374 Kinder aus 37 Klassen in 21 Schulen (Anteil Mädchen $=46.5 \%$, Durchschnittsalter 10.7 Jahre). Weiter liegen Daten aus Fragebogen von 873 Elternteilen und 111 Lehrpersonen vor.

\subsection{Instrumente}

\subsubsection{Leistungstests}

Die Leistungstests in den Promotionsfächern Mathematik und Deutsch bestanden aus einer Auswahl von Aufgaben von Moser, Buff, Angelone und Hollenweger (2011). Zur Auswertung der Tests wurden die Items der jeweiligen Aufgaben dichotom kodiert (falsch: 0 Punkte, richtig: 1 Punkt).

In Tabelle 1 sind die Inhalte und Reliabilitätswerte der Leistungstests in Mathematik und Deutsch zusammenfassend dargestellt. Die Berechnungen beruhen jeweils auf der Gesamtstichprobe des jeweiligen Messzeitpunkts. Die Split-half-Reliabilitäten der Leistungstests sind für beide Fächer und alle Messzeitpunkte zufriedenstellend.

In den Tests beider Fächer wurde in einem Ankeritem-Design eine Teilmenge der Items des früheren Messzeitpunkts zum jeweils folgenden Messzeitpunkt vorgelegt. Basierend auf der Item Response Theorie (IRT; Yen \& Fitzpatrick, 2006) wurde mit der Software R die Linking-Methode von Haberman (2009) angewendet, mit welcher die Schülerleistungen auf einer Metrik abgebildet werden, wodurch die tatsächliche Veränderung der Kompetenzwerte über die drei Messzeitpunkte beschrieben werden kann. Als Kompetenzwerte der Schülerinnen und Schüler wurden sogenannte Weighted-likelihood-Werte (Warm, 1989) ermittelt (Mittelwerte: t1: Mathematik $M=.03, S D=1.09$, Deutsch $M=.04, S D=1.04$; t2: Mathematik 
Tab. 1 Inhalte und Reliabilitätswerte der Leistungstests in Mathematik und Deutsch

\begin{tabular}{|c|c|c|c|}
\hline & \multirow[b]{2}{*}{ Inhalt } & \multicolumn{2}{|c|}{ Split-half-Reliabiliäten } \\
\hline & & $\begin{array}{l}\text { erste Testhälfte vs. } \\
\text { zweite Testhälfte }\end{array}$ & $\begin{array}{c}\text { gerade vs. } \\
\text { ungerade Items }\end{array}$ \\
\hline \multicolumn{4}{|c|}{ Leistungstests Mathematik } \\
\hline Welle 1 & 7 Aufgaben, 39 Items & $r_{\mathrm{SH}}=.80$ & $r_{\mathrm{SH}}=.87$ \\
\hline Welle 2 & 10 Aufgaben, 45 Items & $r_{\mathrm{SH}}=.82$ & $r_{\mathrm{SH}}=.91$ \\
\hline Welle 3 & 8 Aufgaben, 55 Items & $r_{\mathrm{SH}}=.77$ & $r_{\mathrm{SH}}=.91$ \\
\hline \multicolumn{4}{|c|}{ Leistungstests Deutsch } \\
\hline Welle 1 & 6 Aufgaben, 83 Items & $r_{\mathrm{SH}}=.80$ & $r_{\mathrm{SH}}=.92$ \\
\hline Welle 2 & 10 Aufgaben, 85 Items & $r_{\mathrm{SH}}=.82$ & $r_{\mathrm{SH}}=.95$ \\
\hline Welle 3 & 11 Aufgaben, 97 Items & $r_{\mathrm{SH}}=.85$ & $r_{\mathrm{SH}}=.95$ \\
\hline
\end{tabular}

$M=.51, S D=.99 ;$ Deutsch $M=.25, S D=1.13$; 3 : Mathematik: $M=.60, S D=.96$; Deutsch $M=.52, S D=1.18)$.

\subsubsection{Schülerbefragung}

Geschlecht: Das Geschlecht der Schülerinnen und Schüler wurde mit der Angabe „männlich/weiblich“ erhoben.

Schulniveau 7. Klasse: In der 7. Klasse wurde das Schulniveau, in welchem sich die Schülerinnen und Schüler aktuell befanden, erhoben. Falls diese Angabe nicht vorlag, wurde die Information mit der Information der Lehrperson ergänzt. Die Lehrpersonen gaben jeweils am Ende der Primarschule Auskunft, für welche Schulniveaus die Schülerinnen und Schüler angemeldet wurden.

Intelligenz: Die Erfassung der Intelligenz erfolgte mittels der Kurzversion des sprachund kulturunabhängigen Intelligenztests CFT-20-R (1. Testteil; Weiss, 2008). Im Durchschnitt wurden in der Längsschnittstichprobe 36.2 Aufgaben der 56 Aufgaben von den Schülerinnen und Schülern korrekt gelöst. Die individuellen Testwerte wurden an der Normstichprobe der 5. Klasse standardisiert $(M=100, S D=15)$.

\subsubsection{Lehrpersonenbefragung}

Schulniveauerwartung: Im Lehrpersonenfragebogen der ersten Welle gaben die Klassenlehrpersonen für jedes Kind ihrer Klasse an, für welches Schulniveau der Sekundarstufe I sie es empfehlen würden, wenn der Übertritt unmittelbar bevorstünde. Die Antwortmöglichkeiten entsprachen den kantonsspezifischen 
Bezeichnungen und beinhalteten drei bzw. vier mögliche Schulniveaus. Für die hier geplanten Auswertungen wurde die Schulniveauerwartung zuerst in einer dreistufigen Variable abgebildet und nachher in zwei Dummy-Variablen kodiert: Schulniveau mit hohen Anforderungen vs. Schulniveau mit erweiterten und Grundanforderungen sowie Schulniveau mit Grundanforderungen vs. Schulniveau mit erweiterten und hohen Anforderungen.

Im vorliegenden Artikel werden die in der Welle 1 erhobenen Schulniveauerwartungen der Lehrperson für die Gruppe mit spätem Übertritt als nicht kommunizierte Schulniveauerwartungen bezeichnet, da sie zum Erhebungszeitpunkt den Schülerinnen und Schülern sowie deren Eltern noch nicht kommuniziert wurden. Die Schulniveauerwartungen der Lehrperson für die Gruppe mit frühem Übertritt gelten als kommunizierte Schulniveauerwartungen, da das Übertrittsverfahren bereits abgeschlossen war und die Einteilung in das zukünftige Schulniveau mit den Schülerinnen und Schülern sowie den Eltern zum Erhebungszeitpunkt bereits besprochen worden war.

Leistungsbezogene Erwartungen: Die Lehrpersonen der ersten Welle schätzten die Leistungen der Schülerinnen und Schüler in ihren Klassen ein. Gefragt wurde auf einer dreistufigen Skala nach der erbrachten Leistung (unterdurchschnittlich ,1', durchschnittlich ,2' und überdurchschnittlich ,3') in den drei Hauptfächern Mathematik, Deutsch und je nach Kanton Französisch oder Natur-Mensch-Mitwelt im Vergleich zu den übrigen Schülerinnen und Schülern der Klasse $(M=2.09$, $S D=.73, N=873)$.

\subsubsection{Elternbefragung}

Sozioökonomischer Status: Der sozioökonomische Status (ISEI) der Familie wurde anhand der Berufe der ersten und der zweiten Bezugspersonen (Erziehungsberechtigte) zum ersten Messzeitpunkt ermittelt. Die Berufe wurden gemäss der International Standard Occupation Classification (ISCO-08) kodiert. Anschliessend wurde jedem Beruf ein Wert basierend auf dem Standard International Socio-Economic Index of Occupational Status (ISEI-08) zugewiesen (Ganzeboom, 2010). Der jeweils höhere Wert der beiden Bezugspersonen wurde verwendet.

Schulniveauerwartung: Die Eltern wurden zum ersten Messzeitpunkt befragt, welches Schulniveau der Sekundarstufe I ihr Kind nach ihrer Meinung besuchen wird. Kantonsübergreifend erwarteten $13.2 \%$ der Eltern, ihr Kind werde das Niveau mit Grundansprüchen besuchen, $47.1 \%$ erwarteten einen Übertritt in das Niveau mit erweiterten Ansprüchen und 39.7\% der Eltern gaben an, ihr Kind werde voraussichtlich in das Niveau mit hohen Ansprüchen übertreten. 


\subsubsection{Durchführung}

Die schriftlichen Befragungen der Schülerinnen und Schüler, Eltern und Klassenlehrpersonen fanden in den Herbstsemestern der Schuljahre 2011/12, 2012/13 und 2013/14 statt. Die Schülerbefragung und die Leistungstests, die terminlich rund drei Wochen auseinanderlagen, wurden unter Anleitung von geschulten Projektmitarbeitenden im Klassenverband während der Unterrichtszeit durchgeführt. Im Zeitraum zwischen den beiden Klassenbesuchen füllten die Eltern und Lehrpersonen jeweils individuell ihre Fragebogen schriftlich aus.

\section{$3 \quad$ Ergebnisse}

\subsection{Deskriptive Ergebnisse}

Zur Beschreibung der Zusammenhangsmuster der postulierten Prädiktoren wurden getrennt für die zwei Gruppen „früher Übergang“ und „später Übergang“ im nächsten Schritt die Korrelationen berechnet (Tabelle 2). Alle Korrelationen sind signifikant und zum Teil substanziell. Sie stimmen zwischen den zwei Gruppen in hohem Mass überein. Die Schulniveauerwartungen und die Leistungserwartungen von Lehrpersonen korrelierten mit den Leistungen in Mathematik und Deutsch mehrheitlich höher als mit der Intelligenz.

Tab. 2 Pearson-Korrelationen

\begin{tabular}{|c|c|c|c|c|c|c|}
\hline & 1 & 2 & 3 & 4 & 5 & 6 \\
\hline 1 Leistung M 5. Sj & 1 & $.63^{* * *}$ & $.39^{* * *}$ & $.32^{\star \star \star}$ & $-.52^{\star \star \star}$ & $.63^{* * *}$ \\
\hline 2 Leistung D 5. Sj & $.49^{* * *}$ & 1 & $.34^{* * *}$ & $.33^{\star * *}$ & $-.54^{\star \star \star}$ & $.62^{* * *}$ \\
\hline 3 Schülerintelligenz & $.27^{* * *}$ & $.39^{* * *}$ & 1 & $.22^{\star * *}$ & $-.28^{\star * *}$ & $.31^{\star * *}$ \\
\hline 4 Ni-erwart Lp hoch & $.14^{\star * \star}$ & $.35^{\star * *}$ & $.09^{*}$ & 1 & $-.33^{* * *}$ & $.55^{\star * *}$ \\
\hline $5 \mathrm{Ni}$-erwart Lp tief & $-.17^{\star * *}$ & $-.39^{\star * *}$ & $-.27^{\star * *}$ & $-.10^{*}$ & 1 & $-.68^{\star * *}$ \\
\hline 6 Leistungserwart & $.32^{* * *}$ & $.51^{\star * \star}$ & $.24^{\star * *}$ & $.58^{\star \star *}$ & $-.62^{\star * *}$ & 1 \\
\hline
\end{tabular}

Legende: ${ }^{* * *}: p<.001,{ }^{* *}: p<.01,{ }^{\star}: p<.05 ; \mathrm{M}$ : Mathematik; D: Deutsch; Ni-Erwart Lp hoch: von Lehrpersonen erwartete Zuweisung in ein Schulniveau mit hohen Anforderungen; Ni-Erwart Lp tief: von Lehrpersonen erwartete Zuweisung in ein Schulniveau mit Grundanforderungen; Leistungserwart: leistungsbezogene Lehrpersonenerwartungen oberhalb Diagonale: Gruppe mit frühem Übergang $(N=499)$, unterhalb Diagonale: Gruppe mit spätem Übergang $(N=374)$ 


\subsection{Effekte nicht kommunizierter Schulniveauerwartungen}

Zur Überprüfung der Hypothese 1 wurden getrennt für Mathematik und Deutsch stufenweise Regressionsanalysen mit der Stichprobe der Schülerinnen und Schüler mit spätem Übertritt gerechnet. Mit dieser Teilstichprobe liessen sich Effekte von impliziten, nicht kommunizierten Lehrpersonenerwartungen testen (vgl. Operationalisierung im Methodenteil). Es wurden keine Mehrebenenanalysen gerechnet, weil die Klassenzugehörigkeiten beim Übertritt in die Sekundarstufe I wechselten und um immer die gleichen Analysestrategien zu verwenden, damit die Ergebnisse direkt vergleichbar sind. Die Ergebnisse in Tabelle 3 zeigen, dass in Übereinstimmung mit Hypothese la nicht kommunizierte Schulniveauerwartungen von Lehrpersonen für ein Schulniveau mit hohen Anforderungen, die Leistungsveränderung in Mathematik vom 5. zum 6. Schuljahr der Primarschule erklären $\left(R^{2}\right.$ change $\left.=3.0 \%\right)$, auch nach Kontrolle der Intelligenz und des sozioökonomischen Status der Schülerin/des Schülers. Bezieht man die leistungsbezogenen Lehrpersonenerwartungen im Modell mit ein, erklären diese die Leistungsveränderung signifikant $\left(R^{2}\right.$ change $\left.=1.2 \%\right)$. Mit dem Bootstrappingverfahren (Preacher \& Hayes, 2008, 1000 Bootstrapping-Stichproben) wurde die Mediation der dummy-kodierten Variable "Schulniveauerwartung hohe Anforderungen“ von Lehrpersonen (Referenzkategorien Schulniveau mit erweiterten und tiefen Anforderungen) bzw. Schulniveauerwartung tief (mit Referenzkategorien Schulniveau mit erweiterten und hohen Anforderungen) durch die leistungsbezogenen Lehrpersonenerwartungen auf die Leistungsveränderung auf ihre Signifikanz geprüft. Sowohl die Erwartung auf ein Schulniveau mit hohen Anforderungen (Bootstrapintervall .06 bis .22) als auch die Erwartung auf ein Schulniveau mit Grundanforderungen (Bootstrapintervall -.30 bis -.07) wurden gemäss Hypothese 2 a durch die leistungsbezogenen Lehrpersonenerwartungen teilweise aber signifikant (5\%-Niveau) vermittelt. 
Tab. 3 Regressionsanalysen: Nicht kommunizierte Lehrpersonenerwartungen und Schülerleistungen in Mathematik: Gruppe später Übertritt

\begin{tabular}{|c|c|c|c|c|c|c|}
\hline \multirow[b]{2}{*}{ Modell } & \multicolumn{3}{|c|}{ Leistungen M 6. Sj } & \multicolumn{3}{|c|}{ Leistungen $\mathrm{M}$ 7. Sj } \\
\hline & M1 & M2 & M3 & M1 & M2 & M3 \\
\hline Leistungen M 5. Sj & $.61^{* * *}$ & $.53^{\star * *}$ & $.47^{\star \star *}$ & $.50^{\star \star *}$ & $.41^{\star \star \star *}$ & $.39^{* * *}$ \\
\hline IQ $5 . \mathrm{Sj}$ & $.10^{*}$ & .07 & .07 & $.21^{* * *}$ & $.18^{\star * *}$ & $.18^{\star \star \star}$ \\
\hline $\begin{array}{l}\text { Sozioökonomischer } \\
\text { Status (ISEI) }\end{array}$ & $.11^{* *}$ & $.08^{*}$ & .08 & $.10^{*}$ & .07 & .07 \\
\hline Schulni Lp hoch $5 . \mathrm{Sj}$ & & $.17^{\star * *}$ & $.11^{*}$ & & $.14^{* * *}$ & $.12^{\star *}$ \\
\hline Schulni Lp tief $5 . \mathrm{Sj}$ & & -.08 & .00 & & $-.14^{\star *}$ & $-.11^{\star}$ \\
\hline L-Erw Lp 5. Sj & & & $.19^{\star \star}$ & & & .07 \\
\hline$F, p$ & $113.5^{\star * *}$ & $77.2^{\star * *}$ & $67.4^{* * *}$ & $92.0^{* * *}$ & $63.6^{* * *}$ & $53.2^{* * *}$ \\
\hline$d f_{1,} d f_{2}$ & 3,370 & 5,368 & 6,367 & 3,370 & 5,368 & 6,367 \\
\hline$R^{2} a d j$ & $47.5 \%$ & $50.5 \%$ & $51.7 \%$ & $42.3 \%$ & $45.6 \%$ & $45.6 \%$ \\
\hline
\end{tabular}

Legende: M: Mathematik; Schulni Lp: Schulniveauerwartungen Lehrperson; L-Erw Lp: Leistungserwartungen von Lehrpersonen; Sj: Schuljahr; ${ }^{* *}: p<.001 ;{ }^{* *}: p<.01 ;{ }^{*}: p<.05$

Im nächsten Schritt wurde für die gleiche Stichprobe die Leistungsveränderung vom 5. ins 7. Schuljahr der Sekundarstufe I durch die gleichen Prädiktoren erklärt. Die Schulniveauerwartungen wurden gemäss Hypothese la signifikant $\left(R^{2}\right.$ change $=0.8 \%$ ). Anders als bei der Prädiktion der Leistungsentwicklung vom 5. zum 6 . Schuljahr wurden die leistungsbezogenen Lehrpersonenerwartungen nun nicht signifikant. Entgegen Hypothese 2 werden die Schulniveauerwartungen hoch (Bootstrapintervall -.03 bis .13) und Schulniveauerwartungen tief (Bootstrapintervall -.19 bis .04) demnach nicht durch Leistungserwartungen mediiert.

Um die Ergebnisse für ein weiteres Schulfach zu replizieren, wurden die gleichen Analysen für das Fach Deutsch wiederholt. Die Ergebnisse (Tabelle 4) sind auf der Konzeptebene ähnlich, auch wenn sich die einzelnen dummy-kodierten Variablen zur Schulniveauerwartung je nach Analyse in ihrer Signifikanz unterscheiden. Hohe Schulniveauerwartungen erklärten die Leistungsveränderung vom 5. ins 6. Schuljahr in Deutsch signifikant $\left(R^{2}\right.$ change $=0.9 \%$ ). Sie werden durch die leistungsbezogenen Lehrpersonenerwartungen signifikant mediiert (Bootstrapintervall .14 bis .33). Tiefe Schulniveauerwartungen erklärten die Leistungsveränderung vom 5. ins 6. Schuljahr hingegen nicht signifikant. In Bezug auf die Leistungsveränderung vom 5. zum 7. Schuljahr zeigte sich das folgende Bild: Tiefe Schulniveauerwartungen erklärten die Leistungsveränderungen vom 5. ins 7. Schuljahr der Sekundarstufe I $\left(R^{2}\right.$ change $=1.2 \%$ ). Sie wurden im Unterschied zum Fach Mathematik in Übereinstim- 
mung mit Hypothese $2 \mathrm{~b}$ durch die leistungsbezogenen Lehrpersonenerwartungen signifikant mediiert (Bootstrapintervall -.39 bis -.12).

Tab. 4 Nicht kommunizierte Lehrpersonenerwartungen und Schülerleistungen in Deutsch: Gruppe später Übertritt (Regressionsanalysen)

\begin{tabular}{|c|c|c|c|c|c|c|}
\hline \multirow[b]{2}{*}{ Modell } & \multicolumn{3}{|c|}{ Leistungen D 6. Sj } & \multicolumn{3}{|c|}{ Leistungen D 7. Sj } \\
\hline & M1 & M2 & M3 & M1 & M2 & M3 \\
\hline Leistungen D 5. Sj & $.65^{\star * *}$ & $.60^{\star * \star}$ & $.52^{\star * *}$ & $.65^{\star \star *}$ & $.57^{\star \star *}$ & $.52^{\star * *}$ \\
\hline IQ $5 . \mathrm{Sj}$ & .07 & .05 & .04 & $.14^{\star * *}$ & $.12^{\star *}$ & $.11^{\star \star}$ \\
\hline $\begin{array}{l}\text { Sozioökonomischer } \\
\text { Status (ISEI) }\end{array}$ & $.10^{*}$ & $.08^{*}$ & .07 & .01 & -.01 & -.02 \\
\hline Schulni Lp hoch . Sj & & $.10^{\star}$ & .01 & & .04 & -.03 \\
\hline Schulni Lp tief $5 . S j$ & & -.05 & .06 & & $-.14^{\star \star}$ & -.05 \\
\hline L-Erw Lp 5. Sj & & & $.28^{\star * *}$ & & & $.22^{* * *}$ \\
\hline$F, p$ & $122.3^{\star * *}$ & $76.3^{\star * \star}$ & $70.9^{\star * \star}$ & $123.8^{\star * *}$ & $78.4^{* * *}$ & $69.7^{\star * *}$ \\
\hline$d f_{1} d f_{2}$ & 3,370 & 5,368 & 6,367 & 3,370 & 5,368 & 6,367 \\
\hline$R^{2}$ adj & $49.4 \%$ & $50.2 \%$ & $52.9 \%$ & $49.7 \%$ & $50.9 \%$ & $52.5 \%$ \\
\hline
\end{tabular}

Legende: D: Deutsch; Schulni Lp: Schulniveauerwartungen Lehrperson; L-Erw Lp: Leistungserwartungen von Lehrpersonen; $\mathrm{Sj}$ : Schuljahr; ${ }^{* *}: p<.001 ;{ }^{* *}: p<.01 ;{ }^{*}: p<.05$

Die Ergebnisse zeigen mehrheitlich übereinstimmend für Mathematik und Deutsch signifikante Effekte der impliziten, nicht kommunizierten Schulniveauerwartungen, vor allem bei der Leistungsentwicklung vom 5. zum 6. Schuljahr der Primarschule, etwas schwächer vom 5. zum 7. Schuljahr beim späten Übertritt in die Sekundarstufe I. Der Effekt dieser Schulniveauerwartungen wird durch leistungsbezogene Erwartungen von Lehrpersonen mehrheitlich mediiert, mit Ausnahme der Leistungsentwicklung vom 5. zum 7. Schuljahr in Mathematik.

\subsection{Effekte kommunizierter Schulniveauerwartungen}

Vorbereitende deskriptive Analysen mit der Stichprobe mit frühem Übertritt zeigen, dass die kommunizierte Schulniveauerwartung von Lehrpersonen im 5. Schuljahr und das tatsächliche Schulniveau im 6. Schuljahr in $92.0 \%$ der Fälle übereinstimmt, während die Schulniveauerwartung von Eltern dies nur in 89.3 \% der Fälle mit der Zuweisung tut. In der Stichprobe der Jugendlichen mit spätem Übertritt korrespondiert die Schulniveauerwartung der Lehrperson im 5. Schuljahr in $73.5 \%$ der 
Fälle mit dem Schulniveau im 7. Schuljahr, während die Schulniveauerwartung der Eltern in $69.3 \%$ der Fälle übereinstimmt.

Um die Validität der kommunizierten Schulniveauerwartung von Lehrpersonen zu überprüfen, wurden die Übereinstimmungen zwischen der Schulniveauerwartung der Lehrpersonen und Eltern zwischen den beiden Gruppen verglichen. Die Übereinstimmung ist gemäss Hypothese 3 mit $89.8 \%$ in der Gruppe früher Übertritt signifikant höher als in der Gruppe später Übertritt mit 64.1\% $\left(\mathrm{Chi}^{2}=38.8, d f=1\right.$, $p<.001)$. Schulniveauerwartungen von Lehrpersonen in der Gruppe früher Übertritt sind mit den Eltern mehrheitlich abgestimmt worden und kommuniziert. Damit wird die Operationalisierung gestützt, dass Schulniveauerwartungen je nach Stand des Übertrittsverfahrens unterschiedliche Bedeutungen haben.

Zur Prüfung der Hypothesen 4 und 5 wurde auf die Teilstichprobe der Kinder mit frühem Übertritt zurückgegriffen. In dieser Übertrittssituation korrespondieren die Schulniveauerwartungen mit den kommunizierten Schulniveauerwartungen von Lehrpersonen. Es wurden wiederum stufenweise Regressionsanalysen unter Kontrolle der Schülerintelligenz und des sozioökonomischen Status getrennt für Mathematik und Deutsch gerechnet. Gemäss Tabelle 5 und in Übereinstimmung mit Hypothese 4a erklärten kommunizierte Schulniveauerwartungen von Lehrpersonen in Bezug auf die Zuweisung in ein Schulniveau mit Grundansprüchen die Leistungsentwicklung in Mathematik beim Übergang vom 5. Schuljahr der Primarschule ins 6. Schuljahr der Sekundarstufe I signifikant und substanziell $\left(R^{2}\right.$ change $=10.6 \%$ ). Sowohl die Schulniveauerwartungen hoch (Bootstrapintervall -.13 bis .10) als auch die Schulniveauerwartungen tief (Bootstrapintervall -.10 bis .13) wurden gemäss Hypothese 5 a nicht durch leistungsbezogene Lehrpersonenerwartungen mediiert. Gemäss Hypothese $4 \mathrm{~b}$ erklärten kommunizierte hohe und tiefe Schulniveauerwartungen von Lehrpersonen auch die Leistungsentwicklung in Mathematik vom 5. ins 7. Schuljahr signifikant und substanziell $\left(R^{2}\right.$ change $\left.=8.8 \%\right)$. Wiederum wurden die Schulniveauerwartungen hoch (Bootstrapintervall -.11 bis .12) sowie die Schulniveauerwartungen tief (Bootstrapintervall -.12 bis .11) gemäss Hypothese $5 \mathrm{a}$ nicht durch die leistungsbezogenen Lehrpersonenerwartungen mediiert. 
Tab.5 Kommunizierte Lehrpersonenerwartungen und Schülerleistungen in Mathematik: Gruppe früher Übertritt

\begin{tabular}{|c|c|c|c|c|c|c|}
\hline \multirow[b]{2}{*}{ Modell } & \multicolumn{3}{|c|}{ Leistungen M 6. Sj } & \multicolumn{3}{|c|}{ Leistungen $\mathrm{M}$ 7. Sj } \\
\hline & M1 & M2 & M3 & M1 & M2 & M3 \\
\hline Leistungen M 5. Sj & $.46^{* * *}$ & $.41^{\star * *}$ & $.41^{\star * \star}$ & $.44^{* * *}$ & $.39^{* * *}$ & $.39^{* * *}$ \\
\hline IQ 5. Sj & $.33^{* * *}$ & $.27^{\star * \star}$ & $.27^{\star * *}$ & $.32^{\star * *}$ & $.26^{* * *}$ & $.26^{* * *}$ \\
\hline $\begin{array}{l}\text { E: Sozioökonomischer } \\
\text { Status (ISEI) }\end{array}$ & $.25^{* * *}$ & $.08^{*}$ & .08 & .19 & .04 & .04 \\
\hline Schulni Lp hoch $5 . \mathrm{Sj}$ & & .03 & .05 & & $.10^{\star * *}$ & $.11^{\star *}$ \\
\hline Schulni Lp tief $5 . S j$ & & $-.38^{\star \star \star}$ & $-.40^{\star * \star}$ & & $-.33^{\star \star \star}$ & $-.33^{* * *}$ \\
\hline L-Erw Lp 5. Sj & & & -.03 & & & -.02 \\
\hline$F, p$ & $158.4^{* * *}$ & $146.3^{\star * *}$ & $121.9^{* * *}$ & $128.6^{\star * *}$ & $110.4^{* * *}$ & $91.8^{* * *}$ \\
\hline$d f_{1} d f_{2}$ & 3,495 & 5,493 & 6,492 & 3,495 & 5,493 & 6,492 \\
\hline$R^{2} a d j$ & $48.7 \%$ & $59.3 \%$ & $59.3 \%$ & $43.5 \%$ & $52.3 \%$ & $52.2 \%$ \\
\hline
\end{tabular}

Legende: M: Mathematik; Schulni Lp: Schulniveauerwartungen Lehrperson; L-Erw Lp: Leistungserwartungen von Lehrpersonen; Sj: Schuljahr; ${ }^{* *}: p<.001 ;{ }^{* *}: p<.01 ;{ }^{*}: p<.05$

Die analogen Regressionsanalysen wurden erneut auch für das Fach Deutsch durchgeführt (Tabelle 6). Hohe und tiefe kommunizierte Schulniveauerwartungen von Lehrpersonen erklärten nach Kontrolle der Intelligenz und des sozioökonomischen Status die Leistungsentwicklung vom 5. zum 6. Schuljahr der Sekundarstufe I signifikant und substanziell $\left(R^{2}\right.$ change $\left.=10.9 \%\right)$. Die Schulniveauerwartungen hoch (Bootstrapintervall -.16 bis .06) und die Schulniveauerwartungen tief (Bootstrapintervall -.07 bis .16) wurden gemäss Hypothese $5 \mathrm{~b}$ nicht durch die leistungsbezogenen Lehrpersonenerwartungen mediiert. Tiefe kommunizierte Schulniveauerwartungen von Lehrpersonen erklärten gemäss Hypothese $4 \mathrm{~b}$ auch die Leistungsentwicklung vom 5. ins 7. Schuljahr der Sekundarstufe I signifikant und substanziell ( $R^{2}$ change $=9.0 \%$ ). Der Effekt wurde gemäss Hypothese $5 \mathrm{~b}$ durch die leistungsbezogenen Lehrpersonenerwartungen nicht mediiert (Bootstrapintervall -.04 bis .25). 
Tab. 6 Kommunizierte Lehrpersonenerwartungen und Schülerleistungen in Deutsch: Gruppe früher Übertritt

\begin{tabular}{|c|c|c|c|c|c|c|}
\hline \multirow[b]{2}{*}{ Modell } & \multicolumn{3}{|c|}{ Leistungen D 6. Sj } & \multicolumn{3}{|c|}{ Leistungen D 7. Sj } \\
\hline & M1 & M2 & M3 & M1 & M2 & M3 \\
\hline Leistungen D 5. Sj & $.42^{* * *}$ & $.34^{* * *}$ & $.35^{\star * *}$ & $.37^{\star * *}$ & $.27^{\star * *}$ & $.27^{\star * *}$ \\
\hline IQ $5 . \mathrm{Sj}$ & $.24^{* * *}$ & $.20^{* * *}$ & $.20^{* * *}$ & $.19^{\star * *}$ & $.16^{\star * *}$ & $.16^{* * *}$ \\
\hline $\begin{array}{l}\text { E: Sozioökonomischer } \\
\text { Status (ISEI) }\end{array}$ & $.28^{* * *}$ & $.13^{\star * *}$ & $.13^{\star * *}$ & $.24^{\star * *}$ & $.10^{*}$ & $.10^{\star *}$ \\
\hline Schulni Lp hoch $5 . \mathrm{Sj}$ & & $-.06^{\star}$ & -.05 & & .04 & 0.07 \\
\hline Schulni Lp tief $5 . S j$ & & $-.39^{* * *}$ & $-.41^{\star * *}$ & & $-.36^{* * *}$ & $-.40^{* * *}$ \\
\hline L-Erw Lp 5. Sj & & & -.04 & & & -0.06 \\
\hline$F, p$ & $148.3^{* * *}$ & $137.8^{\star * *}$ & $114.8^{\star * \star}$ & $88.3^{* * *}$ & $77.7^{\star * *}$ & $65.0^{\star * *}$ \\
\hline$d f_{1} d f_{2}$ & 3,495 & 5,493 & 6,492 & 3,495 & 5,493 & 6,492 \\
\hline$R^{2} a d j$ & $47.0 \%$ & $57.9 \%$ & $57.8 \%$ & $34.5 \%$ & $43.5 \%$ & $43.50 \%$ \\
\hline
\end{tabular}

Legende: D: Deutsch; Schulni Lp: Schulniveauerwartungen Lehrperson; L-Erw Lp: Leistungserwartungen von Lehrpersonen; $\mathrm{Sj}$ : Schuljahr; ${ }^{* *}: p<.001 ;{ }^{* *}: p<.01 ;{ }^{*}: p<.05$

Die Ergebnisse waren für Mathematik und Deutsch auf der Konzeptebene nahezu analog. Sie zeigten eine hohe Varianzaufklärung der kommunizierten Schulniveauerwartungen von Lehrpersonen. Überdies werden die kommunizierten Schulniveauerwartungen von Lehrpersonen durch die leistungsbezogenen Lehrpersonenerwartungen nicht mediiert.

\section{Diskussion}

Nicht kommunizierte Schulniveauerwartungen von Lehrpersonen beeinflussen die Leistungsverläufe von Schülerinnen und Schülern in Mathematik und Deutsch in der Primarschule und beim Übergang in die Sekundarstufe I, weil damit unterschiedliche, nicht kommunizierte Leistungserwartungen von Lehrpersonen verbunden sind. Die nicht kommunizierten Schulniveauerwartungen haben auf der Konzeptebene in Mathematik einen stärkeren Effekt als in Deutsch, sowohl bei Verbleib bei der gleichen Klassenlehrperson als auch beim Übertritt in die Sekundarstufe I. Je nach Analyse sind die Effekte der Erwartung auf ein Schulniveau mit hohen bzw. tiefen Ansprüchen aber stärker. Diese Befunde lassen sich gut in die Forschung zu den Erwartungseffekten von Lehrpersonen einordnen (Brophy, 1985; Rubie-Davis et al., 2015) und differenzieren das Konzept der Lehr- 
personenerwartung nach dem Objekt Schulniveau und Leistung. Die Wirkung von nicht kommunizierten Schulniveauerwartungen auf die Leistungen wird durch die leistungsbezogenen Lehrpersonenerwartungen vermittelt, ausser für die Leistungsentwicklung vom 5. zum 7. Schuljahr in Mathematik. Offenbar werden im Fach Mathematik im Unterschied zu Deutsch die leistungsbezogenen Lehrpersonenerwartungen von den Schülerinnen und Schülern nicht verinnerlicht, weshalb sie bei einem Lehrpersonenwechsel keine Wirkungen auf die Leistungen haben. Möglicherweise vergeben Lehrpersonen während des Selektionsverfahrens in Mathematik je nach zugewiesenem Schulniveau unterschiedlich anspruchsvolle Aufgaben, so dass die Erwartungseffekte überlagert werden. Viele Lehrpersonen richten ihren Mathematikunterricht an Aufgaben aus, deren Auswahl an die Fähigkeiten der Schülerinnen und Schüler angepasst wird (Reusser \& Pauli, 2003). Diese Art von adaptivem Unterricht ist im Deutschunterricht womöglich seltener, weshalb sich verinnerlichte leistungsbezogene Erwartungseffekte selbst nach dem Schulübertritt nachweisen lassen.

Neu ist die Unterscheidung zwischen den nicht kommunizierten Schulniveauerwartungen und kommunizierten Schulniveauerwartungen. Sie lassen sich mit zwei unterschiedlichen Prozessen erklären. Während der Effekt der nicht kommunizierten Schulniveauerwartungen durch implizite Leistungserwartungen von Lehrpersonen erklärt wird, konnte dieser Effekt bei kommunizierten Schulniveauerwartungen von Lehrpersonen auf Schülerleistungen nicht gefunden werden. Hier spielen vermutlich geplante, anreichernde Unterrichtsstrategien eine Rolle. Erstmals wird gezeigt, dass sich die Leistungsentwicklung von Schülerinnen und Schülern je nach Schulniveauerwartung bereits vor dem Übergang in die Sekundarstufe I unterscheiden. Die Ergebnisse zeigen in die Richtung, dass bereits beim Eintritt in ein Niveau der Sekundarstufe I ein Schereneffekt der Leistungsentwicklung in Deutsch und Mathematik beobachtet werden kann.

Die kommunizierten Schulniveauerwartungen von Lehrpersonen erklären mehr Varianz der Leistungsveränderung in Deutsch und Mathematik als die nicht kommunizierten Schulniveauerwartungen. Dies ist interessant, weil die Schülerinnen und Schüler, die mit kommunizierten Schulniveauerwartungen konfrontiert worden sind, früher die Lehrperson wechselten als die Schülerinnen und Schüler, an die nicht kommunizierte Schulniveauerwartungen gerichtet werden. Offenbar wirken sich explizite Schulniveauerwartungen, begleitet durch entsprechende didaktische Strategien der Lehrperson und von damit korrespondierendem Fähigkeitsselbstkonzept und Leistungsmotivation des Schülers bzw. der Schülerin, stärker auf die Leistungsentwicklung aus als nicht kommunizierte Schulniveauerwartungen.

Einschränkend muss angemerkt werden, dass aufgrund der fehlenden Mediationseffekte nicht erklärt werden kann, wie kommunizierte Schulniveauerwartun- 
gen konkret auf die Leistungsentwicklung wirken. Es wurden dazu didaktische Erklärungsansätze vorgelegt, die gesondert überprüft werden müssen. Auch wurden allfällige Interaktionen von Lehrpersonen- und Elternerwartungen nicht analysiert. Es wäre sinnvoll, in weiterführenden Analysen Effekte der Lehrpersonenerwartungen auf die Motivation und das Fähigkeitsselbstkonzept zu untersuchen. Diese Fragen können in Folgeanalysen bearbeitet werden. Mit der Differenzierung von verschiedenen Arten von Lehrpersonenerwartungen und mit der Vorhersage der kurz- und mittelfristigen Veränderung von Leistungen vor und nach einem Schulübergang - repliziert in zwei Fächern im Schweizer Schulsystem - wurde illustriert, wie Lehrpersonen wirksam die Leistungsentwicklung von Schülerinnen und Schülern beeinflussen.

\section{Literatur}

Baron, R. M., \& Kenny, D. A. (1986). The moderator-mediator variable distinction in Social Psychological Research: Conceptual, strategic, and statistical considerations. Journal of Personality and Social Psychology, 51, 1173-1182.

Baumert, J., Stanat, P., \& Watermann, R. (2006). Schulstruktur und die Entstehung differenzieller Lern- und Entwicklungsmilieus. In J. Baumert, P. Stanat \& R. Waterman (Hrsg.), Herkunftsbedingte Disparitäten im Bildungswesen: Differenzielle Bildungsprozesse und Probleme der Verteilungsgerechtigkeit (S.95-188). Wiesbaden: VS-Verlag.

Brophy, J. E. (1985). Teacher-student interaction. In J. B. Dusek (Ed.), Teacher expectancies (pp. 303-328). Hillsdale, NJ: Erlbaum.

Ditton, H., \& Krüsken, J. (2009). Bildungslaufbahnen im differenzierten Schulsystem Entwicklungsverläufe von Laufbahnempfehlungen und Bildungsaspirationen in der Grundschulzeit. Zeitschrift für Erziehungswissenschaft, 12(1), 74-102.

Dusek, J. B., \& Joseph, A. (1983). The bases of teacher expectation: A meta-analysis. Journal of Educational Psychology, 75(3), 327-346.

Eccles, J., Adler, T. F., Futterman, R., Goff, S. B., Kaczala, C. M., Meece, J. L., \& Midgley, C. (1983). Expectancies, values, and academic behaviors. In J. T. Spence (Ed.), Achievement and achievement motives (pp. 75-146). San Francisco: Freeman.

Eccles, J. S., \& Roeser, R. W. (2011). Schools as developmental contexts during adolescence. Journal of Research on Adolescence, 21(1), 225-241.

Eccles, J. S., Wigfield, A., \& Schiefele, U. (1998). Motivation to succeed. In W. Damon \& N. Eisenberg (Eds.), Handbook of child psychology: Social, emotional, and personality development (Vol. 3, pp. 1017-1095). New York: Wiley.

Englund, M. M., Egeland, B., \& Collins, W. A. (2008). Exceptions to High School dropout predictions in a low-income sample: Do adults make a difference? Journal of Social Issue, 64(1), 77-94. 
Friedrich, A., Flunger, B., Nagengast, B., Jonkmann, K., \& Trautwein, U. (2015). Pygmalion effects in the classroom: Teacher expectancy effects on students' math achievement. Contemporary Educational Psychology, 41, 1-12. doi:10.1016/j.cedpsych.2014.10.006

Ganzeboom, H. B. G., \& Treiman, D. J. (2010). International stratification and mobility file: Conversion tools. Amsterdam: Department of Social Research Methodology.

Glock, S., Kneer, J., \& Kovacs, C. (2013). Preservice teachers' implicit attitudes toward students with and without immigration background: A pilot study. Studies in Educational Evaluation, 39(4), 204-210. doi:10.1016/j.stueduc.2013.09.003

Haberman, S. J. (2009). Linking parameter estimates derived from an item response model through separate calibrations. Princeton, NJ: Educational Testing Service.

Harris, M. J., \& Rosenthal, R. (1985). Mediation of interpersonal expectancy effects: 31 meta-analyses. Psychological Bulletin, 97, 363-386.

Jussim, L., \& Harber, K. D. (2005). Teacher expectations and self-fulfilling prophecies: Knowns and unknowns, resolved and unresolved controversies. Personality and Psychology Review, 9(2), 131-155.

Jussim, L., Eccles, J., \& Madon, S. (1996). Social perception, social stereotypes, and teacher expectations: Accuracy and the quest for the powerful self-fulfilling prophecy. Advances in Experimental Social Psychology, 28, 281-388.

Jussim, L., Harber, K. D., Crawford, J. T., Cain, T. R., \& Cohen, F. (2005). Social reality makes the social mind: Self-fulfilling prophecy, stereotypes, bias, and accuracy. Interaction Studies, 6(1), 85-102.

Jussim, L., Robustelli, S., \& Cain, T. R. (2009). Teacher expectations and self-fulfilling prophecies. Handbook of motivation at school, 349-380.

Ludwig, P. H. (1998). Pygmalioneffekt. In D. H. Rost (Hrsg.), Handwörterbuch Pädagogische Psychologie (S. 415-419). Weinheim: Beltz.

McKown, C., \& Weinstein, R. S. (2008). Teacher expectations, classroom context, and the achievement gap. Journal of School Psychology, 46(3), 235-261. doi:10.1016/j.jsp.2007.05.001

Moser, U., Buff, A., Angelone, D., \& Hollenweger, J. (2011). Nach sechs Jahren Primarschule. Deutsch, Mathematik und motivational-emotionales Befinden am Ende der 6. Klasse. Zürich: Bildungsdirektion Kanton Zürich.

Neuenschwander, M. P. (2014). Selektionsentscheidungen beim Übergang in die Sekundarstufe I und in den Arbeitsmarkt im Vergleich. In M. P. Neuenschwander (Hrsg.), Selektion in Schule und Arbeitsmarkt (S. 63-98). Zürich: Rüegger.

Neuenschwander, M. P., Rottermann, B., Rösselet, S., \& Niederbacher, E. (2015). Bedingungen von erwartungswidrigen Schulniveauzuweisungen beim Übergang in die Sekundarstufe I. Zeitschrift für Soziologie der Erziehung und Sozialisation, 35(3), 285-303.

Preacher, K. J., \& Hayes, A. F. (2008). Asymptotic and resampling methods for estimating and comparing indirect effects. Behavior Research Methods, 40(3), 879-891.

Reusser, K., \& Pauli, C. (2003). Mathematikunterricht in der Schweiz und in weiteren sechs Ländern (TIMSS-Video Studies). Zürich: Universität Zürich.

Rosenthal, R., \& Jacobson, L. (1974). Pygmalion im Unterricht. Weinheim: Belz.

Rosenthal, R. (1968). Experimenter expectancy and the reassuring nature of the null hypothesis decision procedure. Psychological Bulletin Monograph Supplement, 70(6, part 2), 30-47.

Rubie-Davies, C. M., Peterson, E. R., Sibley, C. G., \& Rosenthal, R. (2015). A teacher expectation intervention: Modelling the practices of high expectation teachers. Contemporary Educational Psychology, 40, 72-85. doi:10.1016/j.cedpsych.2014.03.003 
Tenenbaum, H. R., \& Ruck, M. D. (2007). Are teachers' expectations different for racial minority than for European American students? A meta-analysis. Journal of Educational Psychology, 99(2), 253-273. doi:10.1037/0022-0663.99.2.253.

Trouilloud, D. O., Sarrazin, P. G., Martinek, T. J., \& Guillet, E. (2002). The influence of teacher expectations on student achievement in physical education classes: Pygmalion revisited. European Journal of Social Psychology, 32, 591-607.

Warm, T. A. (1989). Weighted likelihood estimation of ability in item response theory. Psychometrika, 54(3), 427-450.

Weiss, R. H. (2008). CFT 20-R. Grundintelligenztest Skala 2 - Revision - (CFT 20-R) mit Wortschatztest und Zahlenfolgentest - Revision (WS/ZF-R). Göttingen: Hogrefe.

Yen, W. M., \& Fitzpatrick, A. R. (2006). Item response theory. In R. L. Brennan (Ed.), Educational Measurement (pp. 111-154). Westport: Praeger Publisher.

Open Access Dieses Kapitel wird unter der Creative Commons Namensnennung 4.0 International Lizenz (http://creativecommons.org/licenses/by/4.0/deed.de) veröffentlicht, welche die Nutzung, Vervielfältigung, Bearbeitung, Verbreitung und Wiedergabe in jeglichem Medium und Format erlaubt, sofern Sie den/die ursprünglichen Autor(en) und die Quelle ordnungsgemäß nennen, einen Link zur Creative Commons Lizenz beifügen und angeben, ob Änderungen vorgenommen wurden.

Die in diesem Kapitel enthaltenen Bilder und sonstiges Drittmaterial unterliegen ebenfalls der genannten Creative Commons Lizenz, sofern sich aus der Abbildungslegende nichts anderes ergibt. Sofern das betreffende Material nicht unter der genannten Creative Commons Lizenz steht und die betreffende Handlung nicht nach gesetzlichen Vorschriften erlaubt ist, ist für die oben aufgeführten Weiterverwendungen des Materials die Einwilligung des jeweiligen Rechteinhabers einzuholen.

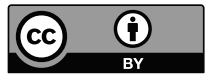




\section{Anpassungsprozesse beim Übergang in die Sekundarstufe I}

Markus P. Neuenschwander

\section{Zusammenfassung}

Die wahrgenommene Passung zwischen Unterrichtsangeboten und Bedürfnissen bzw. Vorwissen der Kinder begünstigt Lern- und Entwicklungsprozesse. Aufgrund der Veränderungsprozesse wird vermutet, dass sich beim Übertritt in die Sekundarstufe I die Passungswahrnehmung, aber auch die Motivations- und Leistungsindikatoren, verschlechtern. In der Längsschnittstudie Wirkungen der Selektion (WiSel) wurden 498 Kinder im 5., 6. und 7. Schuljahr aus 4 Kantonen mit Übertritt nach dem 5. bzw. 6. Schuljahr befragt. Kovarianzanalysen bestätigen die Hypothesen: Passungswahrnehmung, Lern- und Leistungszielorientierung, Noten in Deutsch und Mathematik nehmen bei einem frühen Übertritt in die Sekundarstufe I stärker ab als bei einem späten Übertritt. Die Leistungszunahme in Leistungstests in Deutsch und Mathematik ist beim Übertritt nach dem 5 . Schuljahr geringer als beim Übertritt nach dem 6. Schuljahr. Beim Übertritt korreliert die Stärke der Abnahme der Passungswahrnehmung mit der Veränderung der Noten und der Lern- und Leistungsziele. In Übereinstimmung mit Eccles et al. (1993) wird vermutet, dass sich die Schulorganisation der Sekundarstufe I mit verschiedenen Leistungsniveaus, mit einem Fachlehrpersonensystem und mit stufenspezifischen Lehrplankonzepten weniger günstig auf die Motivations- und Leistungsindikatoren auswirkt als diejenige der Primarstufe ohne Leistungsniveaus und Klassenlehrpersonensystem.

\section{Schlagworte}

Transition, Passung, Leistungen, Noten, Lernzielorientierung, Leistungszielorientierung, Sekundarstufe I 


\section{Ausgangslage}

Der Übertritt in die Sekundarstufe I ist in den Deutschschweizer Kantonen eine normative Schultransition, bei der Kinder in einem standardisierten Selektionsprozess einem Schulniveau mit unterschiedlich hohen schulischen Anforderungen zugewiesen werden. Damit werden erste Entscheidungen für den Bildungsverlauf und den Berufseintritt gefällt. Dieser Übertritt beginnt mit einem Selektionsprozess und endet mit einem Anpassungsprozess der Kinder. Während die Selektionsprozesse beim Übertritt in die Sekundarstufe I seit vielen Jahren intensiv untersucht worden sind (z. B. Maaz, Hausen, McElvany, Baumert, 2006; Neuenschwander \& Malti, 2009), wurden die Anpassungsprozesse nach Eintritt in die Sekundarstufe I, d.h. die Prozesse der Auseinandersetzung der Lernenden mit der neuen Situation in der Sekundarstufe I, weniger intensiv bearbeitet (z. B. Eccles et al., 1993). Dies ist erstaunlich, weil diese Anpassungsprozesse den weiteren Lern- und Entwicklungsprozess der Schülerinnen und Schüler wesentlich steuern. Diese Anpassungsprozesse werden im Folgenden ins Zentrum gestellt. Im vorliegenden Beitrag wird der Frage nachgegangen, wie sich Schülerinnen und Schüler nach dem Übertritt in die Sekundarstufe I an den neuen Kontext anpassen.

\section{$2 \quad$ Passung}

Frühere Forschung zeigte, dass eine hohe Passung zwischen dem Unterricht und den individuellen Bedürfnissen der Kinder eine hohe Motivation und hohe Leistungen in den Schulfächern begünstigt (Zimmer-Gembeck, Chipuer, Hanisch, Creed, McGregor, 2006; Eccles \& Roeser, 2009). Das Ausmass der Passung kann daher als Merkmal von Schulqualität gelten. Passung kann in Weiterführung zu Kristof-Brown, Zimmermann \& Johnson (2005) objektiv als Korrespondenz zwischen den schulischen Angeboten und dem individuellen Lernstand verstanden werden oder schülersubjektiv als wahrgenommene Korrespondenz zwischen dem Unterricht und den eigenen Bedürfnissen und Kompetenzen. Diese subjektive Passung, die im vorliegenden Beitrag im Zentrum steht, drückt sich in der Wahrnehmung aus, die eigenen Stärken im Unterricht gut einbringen zu können, motiviert zu sein, interessante Aufgaben zu erhalten und die eigenen Fähigkeiten zu verbessern. Eine hohe Passungswahrnehmung zwischen Person und Unterricht zeigt sich also darin, dass die Lehrangebote als auf die individuellen Fähigkeiten und Interessen abgestimmt wahrgenommen werden. 
Die Kontexte, welche Jugendliche in Bildungsverläufen durchschreiten, passen unterschiedlich gut zu ihrem Entwicklungsstand. Diese These wurde von Eccles et al. (1993; 2004) mit dem Konzept Stage-environment-fit im US-amerikanischen Raum umschrieben. Eccles (2004) meinte damit, dass die entwicklungsspezifischen Bedürfnisse, Interessen und Kompetenzen (stage) mit der schulischen Umwelt bzw. mit dem Unterricht (environment) korrespondieren sollten, damit sich das Kind optimal entwickeln kann. Diese Passung ist hoch, wenn die schulischen Angebote und Anforderungen optimal auf die alterstypische Situation passen.

Aus einer theoretischen Perspektive beschreibt Passung eine grundlegende Intelligenzleistung im Sinne von Piaget (1947) und Sternberg (1986), um das Überleben eines Menschen zu sichern. Intelligenz meint hier, dass sich Menschen so an eine Umwelt anpassen müssen, dass sie sich optimal entwickeln können. Bezogen auf den Schulkontext schliesst dies einerseits die Veränderung der eigenen Erwartungen an die Anforderungen der Schule, andererseits die Auswahl der passenden Schule sowie der Versuch, die Schule nach den eigenen Bedürfnissen zu gestalten, ein. Passung ist nach dieser Konzeption dynamisch und muss laufend neu hergestellt werden, weil sich sowohl das Individuum als auch die Schule ständig verändern. Mit Passung sind also nicht gemeint, dass Schülerinnen und Schüler konform und gehorsam sind und sich Autoritäten unterordnen.

Das Schaffen einer Passung ist aber auch eine Aufgabe der Schule bzw. des Unterrichts (Eccles et al., 1993). Lehrpersonen stehen im Spannungsfeld, einerseits die Ziele des Lehrplans zu verfolgen und sich andererseits an den Voraussetzungen der Schülerinnen und Schüler zu orientieren. Lehrpersonen unterrichten wirksam, wenn ihre Lernangebote auf die Bedürfnisse und Kompetenzen der Schülerinnen und Schüler abgestimmt sind (vgl. adaptiver Unterricht, Übersicht in Reusser, Pauli, Waldis, 2010). Lehrprozesse erzeugen maximalen Lernerfolg, wenn sie ideal an den Voraussetzungen der Schülerinnen und Schüler ansetzen. Entsprechend forderten Eccles et al. (1993), dass Schulen nicht ausschliesslich Bildung vermitteln, sondern ein Lernumfeld anbieten, das die entwicklungsspezifischen Bedürfnisse und das Wissen der Kinder berücksichtigt (Adaptivität).

\section{$3 \quad$ Lern- und Leistungszielorientierung, Leistung und Noten}

Neben der subjektiven Passungswahrnehmung sind auch Motivation und Leistungen Indikatoren für gelungene Anpassungsprozesse der Kinder an die schulischen Anforderungen. Im Folgenden wird auf die Lern- und Leistungsziele, die als Aspekte 
der Motivation verstanden werden und in den letzten Jahren hohe Aufmerksamkeit erhalten haben, fokussiert. Pintrich, Conley und Kempler (2003) beschrieben die Lern- und Leistungszielorientierung als kognitive Repräsentation davon, was Individuen leisten wollen. Sie sind im Unterschied zu Lebenszielen eher situationsund aufgabenspezifisch. Leistungszielorientierung wird oft in Lernziele (mastery goals) und Leistungsziele (performance goals) differenziert. Lernziele fokussieren auf den Erwerb von Kompetenzen und das Verstehen von Aufgaben. Leistungsziele beschreiben hingegen das Zeigen von Kompetenzen und das Überlegensein gegenüber anderen in der Gruppe (vgl. Neuenschwander, im Druck). Während die Korrelationen zwischen Wichtigkeit von Lernzielen (d.h. Lernzielorientierung) bzw. Leistungszielen (d.h. Leistungszielorientierung) und Leistungstestergebnissen bzw. Noten in der Regel klein sind oder fehlen, erklären Kombinationen von Lern- und Leistungszielorientierung im Jugendalter Leistungstestergebnisse und Noten bedeutsam (Conley, 2012). Die Lern- und die Leistungszielorientierung beschreiben also zwei Aspekte von leistungsrelevanten Einstellungen von Schülerinnen und Schülern, deren Kombination motivationale Haltungen ausdrückt und Leistungsunterschiede der Schülerinnen und Schüler erklärt.

Neben der Passungswahrnehmung und der Lern- und Leistungszielorientierung von Kindern bilden auch die Leistungen von Kindern in Deutsch und Mathematik Indikatoren für den Erfolg von Anpassungsprozessen. Leistungen beschreiben die Ergebnisse von fachspezifischen Leistungstests und sind von Noten, die Leistungsrückmeldungen von Lehrpersonen gegenüber den Kindern widerspiegeln und sich in der Regel am Leistungsniveau der Klasse abbilden, abzugrenzen (z. B. Neuenschwander, 2016). Es wird angenommen, dass Kinder bessere Leistungen in Deutsch und Mathematik erbringen und dadurch bessere Noten in Deutsch und Mathematik erreichen, wenn sie sich an die Angebote und Anforderungen im Unterricht anpassen können.

\section{$4 \quad$ Übertritt in die Sekundarstufe I}

Eccles (2004) berichtete aufgrund einer Analyse von US-amerikanischen Schulen, dass beim Übertritt in die Sekundarstufe I die Passung zwischen dem Kind und dem Unterricht abnimmt, weil die Beziehungen in grossen Schulen anonymer sind und weil die Leistungsanforderungen bei Eintritt in die Highschool zunehmen. Sie postulierte daher eine geringere Passung zwischen den altersspezifischen Bedürfnissen der Kinder und dem schulischen Umfeld in der Sekundarstufe I im Vergleich zur Primarschule. Entsprechend nimmt Eccles (2004) an, dass die 
Lern -und Leistungsorientierung während des Übertritts in die Sekundarstufe I besonders abnehmen und die Leistungszuwächse aufgrund der abnehmenden Passungswahrnehmung geringer werden. Diese These korrespondiert mit Befunden von Alspaugh \& Harting (1995), Alspaugh (1998), Whitley, Lupart \& Beran (2007) sowie van Ophuysen (2008).

Weil die Jugendlichen in der Schweiz mit dem Übertritt in die Sekundarstufe I in ein Schulniveau mit leistungshomogeneren Schulklassen übertreten, ist nicht klar, ob diese These auf die Schule in der Schweiz übertragen werden kann. In der Deutschschweiz treten die Kinder nach der Primarschule in die gegliederte Sekundarstufe I über. Die Kinder werden aufgrund ihrer Leistungen, Elternerwartungen und des Gesamteindrucks der Lehrperson einem Schulniveau zugewiesen. Die Selektionskriterien und der Übertrittszeitpunkt unterscheiden sich zwischen den Kantonen. Während in den Kantonen Aargau und Basel-Landschaft ${ }^{1}$ der Übertritt nach dem 5. Schuljahr stattfindet, vollzieht er sich in den Kantonen Bern und Luzern nach dem 6. Schuljahr.

Analog zur USA sind die Schweizer Schulen in der Sekundarstufe I in der Regel grösser und anonymer als diejenigen der Primarschule. Aufgrund des Fachgruppenlehrpersonensystems in der Sekundarstufe I rücken die persönlichen Beziehungen zwischen Lehrpersonen und Schülerinnen und Schülern im Vergleich zu den Inhalten eher in den Hintergrund. Die Schülerinnen und Schüler müssen sich in einer neuen Schulklasse eine Position/Stellung erwerben (Rösselet \& Neuenschwander, in diesem Band). In der Sekundarstufe I wird der Unterricht weniger individualisiert und stärker lehrerzentriert durchgeführt (Ludwig-Tauber, Wild-Naef, Vouets, 2000; Pauli, Reusser, Waldis, Grob, 2003; Götz, Lohmann, Ganser, Haag, 2005). Daher ist der Übertritt für die Kinder eine Herausforderung, wenn auch in der Regel keine Überforderung (Sirsch, 2000). Es werden die Hypothesen überprüft, wonach die Passungswahrnehmung, Lern- und Leistungszielorientierung abnehmen, die Leistungen und Noten in Deutsch und Mathematik vom 5. zum 7. Schuljahr weniger stark zunehmen. Dabei wird angenommen, dass die Abnahme beim Übertritt in die Sekundarstufe I besonders markant auftritt. Zudem wird die Hypothese getestet, dass die Veränderung der Passungswahrnehmung beim Übertritt in die Sekundarstufe I mit der Veränderung der Lern- und Leistungszielorientierung sowie der Leistungen und Noten in Deutsch und Mathematik zusammenhängt.

1 Der Übergangszeitpunkt wird zukünftig in den Kantonen Aargau und Basel-Landschaft am Ende des 6. Schuljahrs stattfinden. 


\section{$5 \quad$ Methode}

\subsection{Forschungsdesign}

Die Hypothesen werden unter Beizug von Daten aus dem Forschungsprojekt Wirkungen der Selektion ${ }^{2}$ (kurz: WiSel) bearbeitet. Das Projekt wurde in den Kantonen Aargau, Basel-Landschaft, Bern und Luzern durchgeführt. Die vier ausgewählten Kantone unterschieden sich im Übertrittszeitpunkt: In den Kantonen Aargau und Basel-Landschaft war der Übertritt in die Sekundarstufe I nach dem 5. Schuljahr, in den Kantonen Bern und Luzern nach dem 6. Schuljahr (Tabelle 1). Aufgrund dieses quasi-experimentellen Designs kann der Einfluss des Übertritts in die Sekundarstufe I auf die Passungswahrnehmung, die Lern- und Leistungszielorientierung sowie die Leistungen und Noten in Deutsch und Mathematik losgelöst vom Alter untersucht werden.

Tab. 1 Stichprobendesign und Stichprobengrössen

\begin{tabular}{|c|c|c|c|c|c|}
\hline & 5. Klasse & 6. Klasse & 7. Klasse & $N$ (weibl.) & $N$ (männl.) \\
\hline $\begin{array}{l}\text { Früher Übertritt } \\
\text { (Kt AG/BL) }\end{array}$ & Primar & Sek I & Sek I & 117 & 181 \\
\hline $\begin{array}{l}\text { Später Übertritt } \\
\text { (Kt BE/LU) }\end{array}$ & Primar & Primar & Sek I & 95 & 205 \\
\hline
\end{tabular}

Legende: Kt. AG/BL: Kantone Aargau/Basel-Landschaft, Kt. BE/LU: Kantone Bern/Luzern

\subsection{Stichprobe}

Im Jahr 2011 wurden insgesamt 99 Schulklassen mit 1802 Jugendlichen des 5. Schuljahres aus vier Kantonen zufällig ausgewählt. Die Kinder waren durchschnittlich 11-jährig. Diese Jugendlichen wurden im 6. und 7. Schuljahr erneut kontaktiert. Die Längsschnittstichprobe mit Daten zum 5., 6. und 7. Schuljahr umfasst 598 Jugendliche (Tabelle 1). Es wurden keine systematischen Rücklaufverzerrungen gefunden (Neuenschwander, Rösselet, Niederbacher, Rottermann \& Scheffler, 2014).

2 Ich danke dem Schweizerischen Nationalfonds für den finanziellen Beitrag an die Studie (Projektnummer 100013_13459471). Ich danke Dr. Benno Rottermann, Stephan Rösselet und Edith Niederbacher für die Arbeit bei der Durchführung der Studie. 


\subsection{Instrumente}

Es wurden standardisierte Schülerfragebogen und Leistungstests in Deutsch und Mathematik eingesetzt. Es folgt die Beschreibung der Skalen im Schülerfragebogen:

Das Geschlecht wurde mit der folgenden Frage erfasst: „Bitte gib dein Geschlecht an." Als Antwortkategorien dienten (1) weiblich und (2) männlich. Die Schichtzugehörigkeit (ISEI) wurde anhand der Berufe der ersten und der zweiten Bezugspersonen (Erziehungsberechtigte) zum ersten Messzeitpunkt ermittelt. Die Berufe wurden gemäß der International Standard Occupation Classification (ISCO08) codiert (International Labour Organization ILO, 2008). Anschließend wurde jedem Beruf anhand der ISCO-Codierung ein zweistelliger kontinuierlicher Wert basierend auf dem Standard International Socio-Economic Index of Occupational Status (ISEI-08) zugewiesen (Ganzeboom, 2010). Der jeweils höhere Wert der beiden Bezugspersonen wurde verwendet.

Die Passungswahrnehmung wurde mit acht Items operationalisiert, die auf einer 6-Punkte Skala mit den Polen 1 ,stimmt überhaupt nicht' und 6 , stimmt voll und ganz beantwortet werden konnten. Die Eingangsfrage lautete: „Wie sehr stimmen folgende Aussagen zum Unterricht in deiner Klasse für dich?" Die Items begannen mit: „Im Unterricht...“ Sie wurden fortgesetzt zum Beispiel mit „... bin ich sehr motiviert" oder „... erhalte ich interessante Aufgaben“ (Gesamte Stichprobe Welle 1: $\alpha=.82, M=4.84, S D=.65)$. Die Faktorenanalyse ergab einen Faktor, der $38.0 \%$ der Varianz aufklärte.

Die Lernzielorientierung als Indikator der Motivation wurde mit drei Items von Midgley, Maehr, Hruda et al. (2000) gemessen wie zum Beispiel „Mir ist es wichtig, die im Unterricht behandelten Themen vollständig zu verstehen“. Es wurde eine Ratingskala mit 6 Punkten ( 1 , trifft überhaupt nicht zu' und 6 , trifft voll und ganz $\mathrm{zu}$ ) verwendet $(M=5.162, S D=.67, \alpha=.62)$.

Die Leistungszielorientierung als Indikator der Motivation wurde mit drei Items von Midgley et al. (2000) gemessen wie zum Beispiel „Es ist mir wichtig, im Vergleich mit den anderen in meiner Klasse klug zu wirken". Es wurde eine Ratingskala mit 6 Punkten ( 1 , trifft überhaupt nicht zu' bis 6 , trifft voll und ganz zu') verwendet ( $M$ $=3.62, S D=1.19, \alpha=.80$ ).

Die Noten in Deutsch und Mathematik wurden mit der folgenden Frage erfasst: „Wie sind deine Noten? Unten steht eine Liste mit verschiedenen Fächern. Gib bitte für diejenigen Fächer, die du im letzten Jahr besucht hast, deine letzten Zeugnisnoten an. Kreuze bei allen anderen Fächern bitte „keine Note“ an." Es folgte eine Liste mit Schulfächern. Die Noten wurden nach der Schweizer Notenskala erfasst mit den Extremen 1 (sehr schlecht)und 6 (sehr gut), wobei Noten unterhalb von 4 als 
ungenügend bewertet werden. Die Noten wurden zu allen drei Messzeitpunkten rückblickend auf das vergangene Schuljahr erfragt.

Der Mathematiktest der ersten Welle enthielt sieben Konzepte mit insgesamt 39 Items, die dichotom kodiert wurden (falsch: 0 Punkte, richtig: 1 Punkt). Die Split-half-Reliabilitäten erste Testhälfte vs. zweite Testhälfte war $r_{\mathrm{SH}}=.80$, gerade vs. ungerade Items $r_{\mathrm{SH}}=.87$ (Berechnung mit Gesamtstichprobe zu $\mathrm{t}_{1}$ ). Der Mathematiktest der zweiten Welle umfasste zehn Konzepte mit 45 Items. Die Split-half-Reliabilitäten erste Hälfte gegen zweite Hälfte $r_{\mathrm{SH}}=.82$, gerade vs. ungerade Items $r_{\mathrm{SH}}=.91$, Gesamtstichprobe $t_{2}$ waren gut. Der Mathematiktest der dritten Welle bestand aus acht Konzepten mit 55 Items. Die Split-half-Reliabilitäten erste Hälfte gegen zweite Hälfte $r_{\mathrm{SH}}=.77$, gerade vs. ungerade Items $r_{\mathrm{SH}}=.91$, Gesamtstichprobe $\mathrm{t}_{3}$ waren gut (Neuenschwander, Rottermann, Niederbacher, Rösselet \& Scheffler, 2014).

Der Deutschtest der ersten Welle umfasste sechs Konzepte mit insgesamt 83 Items. Analog zum Mathematiktest wurden die Items ebenfalls dichotom mit 0 und 1 kodiert. Die Split-half-Reliabilitäten erste Testhälfte vs. zweite Testhälfte $\underline{\underline{r}}_{\underline{H}}=.80$ und gerade vs. ungerade Items $r_{\mathrm{SH}}=.92$, aus der Gesamtstichprobe Welle 1 waren gut. Der in der zweiten Welle eingesetzte Deutschtest enthielt zehn Konzepte mit insgesamt 85 Items. Die Split-half-Reliabilitäten erste Hälfte gegen zweite Hälfte $r_{\mathrm{SH}}=.82$, gerade vs. ungerade Items $r_{\mathrm{SH}}=.95$, der Gesamtstichprobe $\mathrm{t}_{2}$ waren gut. Der Deutschtest der dritten Welle bestand aus elf Aufgaben mit insgesamt 97 Items. Split-half-Reliabilitäten erste Hälfte gegen zweite Hälfte $r_{\mathrm{SH}}=.85$, gerade vs. ungerade Items $r_{\mathrm{SH}}=.95$, der Gesamtstichprobe $\mathrm{t}_{3}$ waren gut.

In den Tests beider Fächer wurde in einem Ankeritem-Design eine Teilmenge der Items des früheren Messzeitpunkts zum jeweils folgenden Messzeitpunkt vorgelegt. Basierend auf der Item Response-Theorie (IRT; Yen \& Fitzpatrick, 2006) wurde mit der Software R die Linking-Methode von Haberman (2009) angewendet, mit welcher die Schülerleistungen auf einer Metrik abgebildet werden, wodurch die tatsächliche Veränderung der Kompetenzwerte über die drei Messzeitpunkte beschrieben werden kann. Als Kompetenzwerte der Schülerinnen und Schüler wurden sogenannte Weighted-likelihood-Werte (Warm, 1989) ermittelt (Mittelwerte: $t_{1}$ : Mathematik $M=.03, S D=1.09, N=1499$, Deutsch $M=.04, S D=1.04, N=1491 ; \mathrm{t}_{2}$ : Mathematik $M=.51$, $S D=.99, N=1820$, Deutsch $M=.25, S D=1.13, N=1818$; $\mathrm{t}_{3}$ : Mathematik: $M=.60, S D=.96$, $N=1499$, Deutsch $M=.52, S D=1.18, N=1493)$. 


\section{$6 \quad$ Ergebnisse}

\subsection{Veränderung der Passungswahrnehmung}

Um die Hypothese zu prüfen, dass die Passungswahrnehmung mit einem frühen Übertritt von der Primarschule in die Sekundarstufe I stärker sinkt als bei Gleichaltrigen, die später in die Sekundarstufe I übertreten, wurden Kovarianzanalysen mit Messwiederholung gerechnet. Es wurde der Verlauf der Passungswahrnehmung vom 5. zum 7. Schuljahr zwischen der Gruppe mit einem Übertritt in die Sekundarstufe I nach dem 6. Schuljahr (später Übertritt) und der Gruppe mit einem Übertritt in die Sekundarstufe I nach dem 5. Schuljahr (früher Übertritt) verglichen. Ein signifikanter Interaktionseffekt zeigt, dass sich die Verläufe zwischen den zwei Gruppen unterscheiden. Ein linearer Interaktionseffekt zeigt, dass sich die Mittelwertverläufe je nach Übertrittszeitpunkt unterscheiden. Ein quadratischer Interaktionseffekt indiziert, dass sich die Mittelwerte vor bzw. nach dem Übertritt stärker bzw. schwächer als während des Übertritts und im Vergleich zur anderen Gruppe verändern. D.h. , der Übertritt führt zu einer spezifischen $\mathrm{Zu}$ - oder Abnahme eines Merkmals. Es werden die linearen und die quadratischen Effekte ausgewiesen, um die Art der Veränderung des jeweiligen Merkmals präziser beschreiben zu können.

Die beiden Gruppen können sich aufgrund kantonsspezifischer Merkmale unterscheiden, so dass Effekte nicht eindeutig als Effekte des Übertrittszeitpunkts interpretiert werden können. Daher werden die Daten der Jugendlichen aus je zwei Kantonen, in denen die Jugendlichen zum gleichen Zeitpunkt übertreten, zusammengefasst ausgewertet. Überdies werden Kovariaten einbezogen, welche einen Einfluss auf die abhängige Variable haben können und in denen sich die beiden Gruppen unterscheiden können. Konkret werden die Kovariaten Geschlecht, Schicht der Eltern, Nationalität des Kindes sowie die vom Jugendlichen eingeschätzte Wichtigkeit der Schule kontrolliert. Mit diesen Kovariaten wird ein allfälliger Störeffekt von dritten Variablen auf diese Verläufe kontrolliert.

Tab. 2 Veränderung der Passungswahrnehmung: Geschätzte Mittelwerte mit Standardfehlern

\begin{tabular}{lcc}
\hline & früher Übertritt $(N=171)$ & später Übertritt $(N=293)$ \\
\hline 5. Klasse & $4.82(.04)$ & $4.84(.03)$ \\
6. Klasse & $4.73(.05)$ & $4.80(.04)$ \\
7. Klasse & $4.56(.05)$ & $4.72(.04)$ \\
\hline
\end{tabular}

Kovariaten: Geschlecht, Schicht der Eltern, Nationalität des Kindes, vom Jugendlichen eingeschätzte Wichtigkeit der Schule 
Der Haupteffekt Übertrittszeitpunkt war nicht signifikant ( $F$ übertrittszeitpunkt $(1$, $458)=3.1)$, der lineare Zeiteffekt war signifikant $\left(F_{\text {linear Zeit }}(1,458)=21.5, p<.001, F\right.$ quadr Zeit $(1,458)=2.4, n s)$, der lineare Interaktionseffekt war auch signifikant $\left(F_{\text {linear }}\right.$ Interaktion $\left.(1,458)=5.0, p<.05, F_{\text {quadr Interaktion }}(1,458)=0.09, n s\right)$. Die Mittelwerte in Tabelle 2 zeigen in beiden Gruppen ein deutlicheres Sinken der Passungswahrnehmung beim Übertritt und nach Eintritt in die Sekundarstufe I als in der Primarschule. Während die Passungswahrnehmung bei der Gruppe mit spätem Übertritt vom 5. zum 6. Schuljahr in der Primarschule wenig abnimmt, nimmt sie bei den gleichaltrigen Jugendlichen in der Gruppe mit frühem Übertritt stärker ab. Auch vom 6. zum 7. Schuljahr ist die Abnahme in der Gruppe später Übertritt, wenn die Kinder den Übertritt in die Sekundarstufe I vollziehen, kleiner als in der Gruppe der Gleichaltrigen mit frühem Übertritt, welche sich in der Sekundarstufe I befinden. Weil die Jugendlichen mit dem Übertritt in die Sekundarstufe I in unterschiedliche Schulniveaus übertreten, wurde in Kontrollanalysen ein Effekt des Schulniveaus überprüft. Diese Analysen zeigen für die Passungswahrnehmung keinen signifikanten Effekt. Die Ergebnisse stützen die formulierte Hypothese, dass die Passungswahrnehmung in der Gruppe mit frühem Übertritt in die Sekundarstufe I stärker sinkt als in der Gruppe mit spätem Übertritt.

\subsection{Veränderung der Lern- und Leistungszielorientierung}

Im nächsten Schritt wird die Hypothese überprüft, ob analog zur Passungswahrnehmung die Lernziel- und die Leistungszielorientierung in der Gruppe mit frühem Übertritt stärker sinken als in der Gruppe mit spätem Übertritt. Es wurden einfaktorielle Kovarianzanalysen mit Messwiederholung gerechnet. Als Kovariaten wurden Geschlecht, Schicht der Eltern, Nationalität des Kindes sowie die vom Jugendlichen eingeschätzte Wichtigkeit der Schule verwendet. Bei der Lernzielorientierung war der Haupteffekt Übertrittszeitpunkt nicht signifikant $\left(F_{\text {Übertrittszeitpunkt }}(1,451)=1.5, n s\right)$, der lineare und der quadratische Zeiteffekt waren signifikant $\left(F_{\text {linear Zeit }}(1,451)=8.3, p<.01 ; F_{\text {quadr Zeit }}(1,451)=6.5, p<.05\right)$ und der lineare Interaktionseffekt war auch signifikant $\left(F_{\text {linear Interaktion }}(1,451)=7.1, p<.01\right.$; $\left.F_{\text {quadr Interaktion }}(1,451)=.02, n s\right)$. Gemäss der Mittelwerte in Tabelle 3 nimmt analog zur Passungswahrnehmung die Lernzielorientierung in der Gruppe mit frühem Übertritt stärker ab als in der Gruppe mit spätem Übertritt. 
Tab. 3 Veränderung der Leistungen in Deutsch und Mathematik (Mittelwerte und Standardfehler)

\begin{tabular}{lcccc}
\hline & \multicolumn{2}{c}{$\begin{array}{c}\text { Lernzielorientierung } \\
\text { früher Übertritt } \\
N=169\end{array}$} & $\begin{array}{c}\text { später Übertritt } \\
N=288\end{array}$ & $\begin{array}{c}\text { Leistungszielorientierung } \\
\text { früher Übertritt } \\
N=169\end{array}$ \\
$\begin{array}{lcccc}\text { später Übertritt } \\
N=286\end{array}$ \\
\hline 5. Klasse & $5.14(.04)$ & $5.10(.03)$ & $3.51(.09)$ & $3.41(.07)$ \\
6. Klasse & $5.02(.05)$ & $5.09(.04)$ & $3.25(.10)$ & $3.31(.07)$ \\
7. Klasse & $4.78(.05)$ & $4.94(.04)$ & $3.23(.10)$ & $2.98(.07)$ \\
\hline
\end{tabular}

Kovariaten: Geschlecht, Schicht der Eltern, Nationalität des Kindes, vom Jugendlichen eingeschätzte Wichtigkeit der Schule

Bei der Leistungszielorientierung war der Haupteffekt Übertrittszeitpunkt nicht signifikant $\left(F_{\text {Úbertrittszeitpunkt }}(1,447)=1.0, n s\right)$, der lineare und quadratische Zeiteffekt war nicht signifikant $\left(F_{\text {linear Zeit }}(1,447)=.1, n s ; F_{\text {quadr Zeit }}(1,447)=.6\right.$, ns), der lineare Interaktionseffekt war nicht signifikant $\left(F_{\text {linear Interaktion }}(1,447)=1.4 n s\right)$, aber der quadratische Interaktionseffekt war signifikant $\left(F_{\text {quadratisch Interaktion }}(1,447)=5.6\right.$, $p<.05)$. Kontrollanalysen zeigen, dass die Ergebnisse dieser Analysen unabhängig vom Schulniveau sind, das die Schülerinnen und Schüler in der Sekundarstufe I besuchen. Die Mittelwerte in Tabelle 3 zeigen, dass die Leistungszielorientierung in der Gruppe mit frühem Übertritt vor allem vom 5. zum 6. Schuljahr, das heisst beim Übertritt in die Sekundarstufe I, abnimmt und dass sie in der Gruppe später Übertritt vor allem vom 6. zum 7. Schuljahr abnimmt, d. h. beim Übertritt von der Primarschule in die Sekundarstufe I. Die Abnahme der Leistungszielorientierung trat also bei gleichem Alter der Schülerinnen und Schüler vor allem beim Übertritt von der Primarschule in die Sekundarstufe I auf. Analog zur Passungswahrnehmung nahmen auch die Lern- und Leistungszielorientierung besonders beim Übertritt in die Sekundarstufe I ab.

\subsection{Veränderung der Leistungen und der Noten}

Im nächsten Schritt wurde überprüft, ob sich analog zur Passungswahrnehmung die Leistungen in Deutsch und Mathematik veränderten. Wiederum wurden Kovarianzanalysen mit Messwiederholung gerechnet. Als Kovariaten wurden Geschlecht, Schicht der Eltern, Nationalität des Kindes sowie die vom Jugendlichen eingeschätzte Wichtigkeit der Schule verwendet. Für die Leistungen in Deutsch war der Haupteffekt nicht signifikant $\left(F_{\text {Ubbertrittszeitpunkt }}(1,436)=.0, n s\right)$, der lineare Zeiteffekt war nicht signifikant $\left(F_{\text {linear Zeit }}(1,436)=3.3, n s ; F_{\text {quadr Zeit }}(1,436)=.2, n s\right)$, der lineare 
Interaktionseffekt war signifikant $\left(F_{\text {linear Interaktion }}(1,446)=9.0, p<.01 ; F_{\text {quadr Interaktion }}(1\right.$, 446)=1.8, ns). Gemäss Tabelle 4 nahmen die Testleistungen Deutsch in der Gruppe mit spätem Übertritt deutlich stärker zu als in der Gruppe mit frühem Übertritt.

Tab. 4 Veränderung der Leistungen in Deutsch und Mathematik (Mittelwerte und Standardfehler)

\begin{tabular}{|c|c|c|c|c|}
\hline & \multicolumn{2}{|c|}{ Deutsch } & \multicolumn{2}{|c|}{ Mathematik } \\
\hline & $\begin{array}{c}\text { früher Übertritt } \\
\qquad N=158\end{array}$ & $\begin{array}{c}\text { später Übertritt } \\
\qquad N=285\end{array}$ & $\begin{array}{c}\text { früher Übertritt } \\
\qquad N=160\end{array}$ & $\begin{array}{c}\text { später Übertritt } \\
N=286\end{array}$ \\
\hline 5. Kl. & $.31(.07)$ & $.15(.06)$ & $.30(.08)$ & $-.01(.06)$ \\
\hline 6. Kl. & $.31(.08)$ & $.38(.06)$ & $.53(.07)$ & $.56(.05)$ \\
\hline 7. Kl. & $.59(.09)$ & $.70(.06)$ & $.58(.08)$ & $.68(.06)$ \\
\hline
\end{tabular}

Kovariaten: Geschlecht, Schicht der Eltern, Nationalität des Kindes, vom Jugendlichen eingeschätzte Wichtigkeit der Schule

Für die Leistungen in Mathematik war der Haupteffekt nicht signifikant ( $F$ übertrittszeitpunkt $(1,439)=.5, n s)$, der lineare Zeiteffekt war signifikant $\left(F_{\text {linear Zeit }}(1,439)=5.4\right.$, $\left.p<.05 ; F_{\text {quadr Zeit }}(1,439)=.6, n s\right)$ und der lineare und quadratische Interaktionseffekt war auch signifikant $\left(F_{\text {linear Interaktion }}(1,439)=20.3, p<.001 ; F_{\text {quadr Interaktion }}(1,439)=4.8\right.$, $p<.05)$. Kontrollanalysen zeigen allerdings, dass die Veränderung der Leistungen in Deutsch und Mathematik vom Schulniveau, das die Schülerinnen und Schüler in der Sekundarstufe I besuchen, abhängt. Die Leistungszunahme ist beim Übertritt in ein Schulniveau der Sekundarstufe I mit hohen Anforderungen grösser als beim Übertritt in ein Schulniveau mit Grundanforderungen. Auch nach Kontrolle des Schulniveaus nehmen die Leistungen in Deutsch und Mathematik in der Gruppe mit spätem Übertritt deutlich stärker zu als in der Gruppe mit frühem Übertritt. Die Leistungszunahme in der Primarschule war grösser als beim Übertritt in die Sekundarstufe I und die Leistungszunahme beim Übertritt in die Sekundarstufe I war grösser als beim Verbleib in der Sekundarstufe I. Die Ergebnisse zeigen, dass die Leistungen für Deutsch und Mathematik im 7. Schuljahr bei den Jugendlichen mit spätem Übertritt höher sind als bei den Jugendlichen mit frühem Übertritt. Ein später Übertritt in die Sekundarstufe I begünstigt in Übereinstimmung mit den Hypothesen höhere Leistungen in Deutsch und Mathematik.

Um die Veränderung der Noten in Deutsch und Mathematik beim Übertritt in die Sekundarstufe zu beschreiben, wurden Kovarianzanalysen mit Messwiederholung gerechnet. Als Kovariaten wurden wiederum Geschlecht, Schicht der Eltern, Nationalität des Kindes sowie die vom Jugendlichen eingeschätzte Wichtigkeit 
der Schule verwendet. Die Noten liegen für das 4., 5. und 6. Schuljahr vor. Gemäss Tabelle 5 weisen die Schülerinnen und Schüler mit spätem Übertritt bessere Noten in Deutsch als die anderen Schülerinnen und Schüler auf ( $F$ Übertrittszeitpunkt (1, $435)=16.0, p<.001)$. Der Zeiteffekt ist nicht signifikant $\left(F_{\text {linear Zeit }}(1,435)=1.8\right.$, ns; $F$ quadr Zeit $(1,435)=.2, n s)$, die Interaktion Übertrittszeitpunkt mal Zeit ist signifikant $(F$ linear Interaktion $\left.(1,435)=13.2, p<.001 ; F_{\text {quadr Interaktion }}(1,435)=28.3, p<.001\right)$. Auch bei den Noten in Mathematik war der Übertrittszeitpunkteffekt signifikant ( $F_{\text {Ubertrittszeitpunkt }}$ $(1,437)=11.0, p<.001)$, der Zeiteffekt zeigte keine Abnahme der Noten in Mathematik $\left(F_{\text {linear Zeit }}(1,437)=.33, n s ; F_{\text {quadr Zeit }}(1,437)=1.0, n s\right)$. Die Interaktion belegte hypothesenkonform eine stärkere Abnahme der Noten nach dem Übertritt in die Sekundarstufe I in der 5. Klasse zur 6. Klasse als bei den Gleichaltrigen mit Verbleib in der Primarstufe $\left(F_{\text {linear Interaktion }}(1,437)=6.9, p<.01 ; F_{\text {quadr Interaktion }}(1,437)=27.9\right.$, $p<.001)$. Wird das besuchte Schulniveau in der Sekundarstufe I als zusätzlicher Faktor einbezogen, verändern sich diese Effekte bezüglich der Noten in Deutsch und Mathematik nicht. Die Interaktion Übertrittszeitpunkt mal Schulniveau mal Zeit wird in beiden Fächern signifikant. Beim Übertritt in ein Schulniveau mit tiefen Anforderungen nehmen die Noten zu, während sie beim Übertritt in ein Schulniveau mit höheren Anforderungen abnehmen. In der Gruppe mit frühem Übertritt zeigt sich dieser Effekt vom 5. zum 7. Schuljahr, während er bei der Gruppe mit spätem Übertritt nur vom 6. zum 7. Schuljahr auftritt.

Tab. 5 Veränderung der Noten in Deutsch und Mathematik (Mittelwerte und Standardfehler)

\begin{tabular}{lcccc}
\hline & \multicolumn{2}{c}{ Deutsch } & \multicolumn{2}{c}{ Mathematik } \\
& $\begin{array}{c}\text { früher Übertritt } \\
N=160\end{array}$ & $\begin{array}{c}\text { später Übertritt } \\
N=281\end{array}$ & $\begin{array}{c}\text { früher Übertritt } \\
N=161\end{array}$ & $\begin{array}{c}\text { später Übertritt } \\
N=282\end{array}$ \\
\hline $4 . \mathrm{Kl}$. & 4.99 & 5.12 & 4.95 & 5.11 \\
$5 . \mathrm{Kl}$. & 4.99 & 5.03 & 4.93 & 4.97 \\
$6 . \mathrm{K} 1$. & 4.72 & 5.04 & 4.59 & 4.90 \\
\hline
\end{tabular}

Kovariaten: Geschlecht, die Schicht der Eltern, die Nationalität des Kindes sowie die vom Jugendlichen eingeschätzte Wichtigkeit der Schule

\subsection{Korrelationen}

Um den Zusammenhang zwischen der Passungswahrnehmung und den Leistungsindikatoren zu testen, wurden Korrelationsanalysen gerechnet. Die Passungswahrnehmung korrelierte wie erwartet mit den Leistungen in Mathematik ( $r=.10$, 
$p<.05)$, mit den Noten im 5. Schuljahr in Deutsch $(r=.12, p<.01)$ und Mathematik $(r=.20, \mathrm{p}<.001)$ sowie mit der Lern- $(r=.15, p<.001)$ und Leistungszielorientierung $(r=.54, p<.001)$ signifikant. Einzig die Korrelation der Passungswahrnehmung mit den Leistungen in Deutsch war nicht signifikant $(r=.04, n s)$. Die Passungswahrnehmung hängt also erwartungsgemäss mit Indikatoren der Motivation und Leistungen zusammen.

Im nächsten Schritt wurde überprüft, ob das Ausmass der Veränderung der Passungswahrnehmung vom 5. zum 6. Schuljahr mit dem Ausmass der Veränderung der Leistungen in Mathematik und Deutsch, in den Noten in Mathematik und Deutsch und in den Motivationsindikatoren Leistungszielorientierung und Lernzielorientierung zusammenhingen. Wenn die präsentierten Veränderungen der Leistungen, Noten und Motivation beim Übertritt in die Sekundarstufe I durch die Passungswahrnehmung erklärt werden könnten, müsste es systematische Kovariationen in der Differenz der Passungswahrnehmung vom 5. zum 6. Schuljahr mit der Differenz der genannten Leistungsindikatoren vom 5. zum 6. Schuljahr geben. Weil die Noten im 7. Schuljahr nicht vorliegen, wurde diese Analyse nur für die Veränderung vom 5. zum 6. Schuljahr gerechnet, aber getrennt für die Gruppen mit Übertritt und ohne Übertritt. Die Ergebnisse sind in Tabelle 6 dargestellt.

Tab. 6 Pearson-Korrelationen: Veränderung der Passungswahrnehmung und Veränderung der Leistungen und Noten in Deutsch und Mathematik und der Leistungs- und Lernzielorientierung vom 5. zum 6. Schuljahr in den Gruppen früher Übertritt und später Übertritt.

\begin{tabular}{lcccccc}
\hline Passung & $\begin{array}{c}\text { Leistung } \\
\mathrm{M}\end{array}$ & $\begin{array}{c}\text { Leistung } \\
\mathrm{D}\end{array}$ & $\begin{array}{c}\text { Note } \\
\mathrm{M}\end{array}$ & $\begin{array}{c}\text { Note } \\
\mathrm{D}\end{array}$ & $\begin{array}{c}\text { Leistungs- } \\
\text { ziel }\end{array}$ & $\begin{array}{c}\text { Lernziel } \\
\text { Übertritt }\end{array}$ \\
$\begin{array}{l}(184<N<198) \\
\text { Kein Übertritt }\end{array}$ & .13 & -.12 & $.21^{* *}$ & $.20^{* *}$ & $.19^{* *}$ & $.39^{* * *}$ \\
$(322<N<330)$ & .10 & .04 & -.08 & .10 & .07 & $.28^{* * *}$ \\
\hline
\end{tabular}

Legende: ${ }^{* * *}: p<.001,{ }^{* *}: p<.01,{ }^{*}: p<.05$

Beim Übertritt von der Primarschule in die Sekundarstufe I gibt es hypothesenkonform signifikante positive Korrelationen zwischen der Veränderung der Passungswahrnehmung und der Veränderung der Noten in Mathematik und Deutsch, aber auch der Leistungszielorientierung und Lernzielorientierung. Es gibt aber keine signifikanten Korrelationen mit der Veränderung der Leistungen im Mathematikund Deutschtest. In der Gruppe ohne Übertritt vom 5. zum 6. Schuljahr findet sich nur eine signifikante Korrelation (Veränderung der Passungswahrnehmung 
mit Veränderung der Lernzielorientierung). Möglicherweise ist die Varianz der Differenzwerte in der Gruppe ohne Übertritt zu klein, weil sich die Leistungen, Noten und Lern- und Leistungszielorientierung vom 5. zum 6. Schuljahr kaum verändern (vgl. Tabellen 2-5).

Diese deskriptiven Analysen zeigen, dass die Veränderungen der Noten und der Lern- und Leistungszielorientierung beim Übertritt in die Sekundarstufe mit der Veränderung der Passungswahrnehmung systematisch kovariieren. Mit dem Absinken der Passungswahrnehmung in die Sekundarstufe I sinken auch die Lernund Leistungszielorientierung und der Noten.

\section{Schlussfolgerungen}

Die Ergebnisse zeigen in Übereinstimmung mit Eccles (2004), dass analog zur USA auch in der Schweiz die Passungswahrnehmung beim frühen Übertritt in die Sekundarstufe I bei gleichem Alter der Kinder stärker abnimmt als bei einem späten Übertritt. Damit korrespondieren die Befunde, dass beim Übertritt in die Sekundarstufe I auch die Lern- und die Leistungszielorientierung deutlich stärker abnehmen und die Leistungen in Deutsch und Mathematik weniger zunehmen. Das ist nicht primär ein Alterseffekt, sondern ein Effekt der institutionellen Strukturierung und der Lehrpraxis im Unterricht. Die geringere Leistungszunahme in der Sekundarstufe I im Vergleich zur Primarschule berichteten auch Angelone, Keller \& Moser (2013) sowie Moser, Oostlander, Tomasik (in diesem Band). Sie erklärten diese mit der unterschiedlichen Struktur der Lehrpläne in der Primarschule im Vergleich zur Sekundarstufe I.

Diese deskriptiven Ergebnisse lassen keine kausalen Erklärungen zu, wonach die Motivationsabnahme bzw. die geringere Leistungszunahme auf die geringere Passung zwischen individuellen Interessen und Wissen und den Unterrichtsangeboten zurückzuführen ist. Dennoch gibt es beim Übertritt in die Sekundarstufe I zwischen den Veränderungen der Passungswahrnehmung und der Leistungsindikatoren systematische Zusammenhänge. Dies stützt die Interpretation, dass die Veränderung der Noten und der Lern- und Leistungszielorientierung mit der Abnahme der Passungswahrnehmung beim Übertritt in die Sekundarstufe I erklärt werden kann.

Die Gründe für die Abnahme der Passungswahrnehmung sind unzureichend untersucht. Ausgehend von Eccles (2004) Analyse zu US-amerikanischen Schulen wird vermutet, dass in der Sekundarstufe I der Unterricht weniger entwicklungsangepasst ist als in der Primarstufe. Der Unterricht ist fachorientiert und berücksichtigt die individuellen Interessens- und Wissensvoraussetzungen weniger stark 
als in der Primarschule. Die häufigste Unterrichtsform auf der Sekundarstufe I ist der lehrerzentrierte Unterricht (Ludwig-Tauber et al., 2000), der vermutlich die individuellen Interessens- und Vorwissenslagen der Schülerinnen und Schüler unzureichend aufnimmt. Möglicherweise ist der Unterricht in der Sekundarstufe I weniger adaptiv an den Interessen und am Wissen der Kinder ausgerichtet als in der Primarschule. Es könnte sein, dass es in der Sekundarstufe I eine ausgewogenere Balance zwischen den fachlichen und pädagogischen Zielen der Lehrperson und den Bedürfnissen der einzelnen Schülerinnen und Schüler braucht.

Interessant ist, dass sich die Gruppierung der Kinder in leistungshomogene Schulklassen aufgrund der Einteilung in Schulniveaus nicht in einer höheren Passungswahrnehmung, Motivation und Leistungszunahme auswirkt. Die Hoffnung, die Lernbedingungen durch eine Leistungsgruppierung in Schulniveaus zu verbessern, wird innerhalb von 1.5 Jahren nach Eintritt in die Sekundarstufe I nicht erfüllt.

Einschränkend muss angemerkt werden, dass die formulierten Erklärungen zur Veränderung von Passung, Motivation und Leistungsindikatoren beim Übertritt in die Sekundarstufe I nicht spezifisch überprüft werden konnten. Die Ergebnisse belegen je nach Übertrittszeitpunkt unterschiedliche Verläufe, über deren Erklärung aber nur spekuliert werden kann. Die Erklärung dieser Übergangsmuster sollte in zukünftigen Studien überprüft werden. Die Befunde werden gleichwohl als wichtig erachtet, weil sie neu sind und das Verständnis des Übertritts in die Sekundarstufe I vertiefen.

\section{Literatur}

Alspaugh, J. W., \& Harting, R. D. (1995). Transition effects of school grade-level organization on student achievement. Journal of Research and Development in Education, 28, 145-149.

Alspaugh, J. W. (1998). Achievement loss associated with the transition to middle school and high school. Journal of Educational Research, 92, 20-26.

Angelone, D., Keller, F., \& Moser, U. (2013). Entwicklung schulischer Leistungen während der obligatorischen Schulzeit. Bericht zur vierten Zürcher Lernstandserhebung zuhanden der Bildungsdirektion des Kantons Zürich. Zürich: Institut für Bildungsevaluation.

Conley, A. M. (2012). Patterns of motivation beliefs: Combining achievement goal and expectancy-value perspectives. Journal of Educational Psychology, 104(1), 32-47. doi:10.1037/a0026042

Eccles, J. S., \& Roeser, R. W. (2009). Schools, academic motivation, and stage-environment fit. In R. M. Lerner \& L. Steinberg (Eds.), Handbook of adolescent psychology (3rd ed., pp. 404-434). Hoboken, N.J: John Wiley \& Sons. 
Eccles, J. S. (2004). Schools, academic motivation, and stage-environment fit. In R. M. Lerner \& L. Steinberg (Eds.), Handbook of adolescent psychology (2nd ed., Vol. 2, pp. 125-153). Hoboken, NJ: Wiley.

Eccles, J. S., Midgley, C., Wigfield, A., Buchanan, C. M., Reuman, D., Flanagan, C., \& Mac Iver, D. (1993). Development during adolescence: the impact of stage-environment fit on young adolescents' experiences in schools and in families. American Psychologist, 48(2), 90-101.

Ganzeboom, H. B. G., \& Treiman, D. J. (2010). International stratification and mobility file: Conversion Tools. Amsterdam: Department of Social Research Methodology.

Götz, T., Lohrmann, K., Ganser, B., \& Haag, L. (2005). Einsatz von Unterrichtsmethoden Konstanz oder Wandel? Empirische Pädagogik, 19(4), 342-360.

Haberman, S. J. (2009). Linking parameter estimates derived from an item response model through separate calibrations. ETS Research Report No. RR-09-40. Princeton, NJ: ETS.

Kristof-Brown, A. L., Zimmermann, R. D., \& Johnson, E. C. (2005). Consequences of individual's fit at work: A meta-analysis of person-job, person-organization, person-group, and person-supervisor fit. Personnel Psychology, 58, 1-342.

Ludwig-Tauber, M., Wild-Naef, M., \& Vouets, V. (2000). Merkmale des Berufsfeldes von Lehrerinnen und Lehrern der 7. bis 9. Klasse. Eine Studie im Auftrag der Erziehungsdirektion des Kantons Bern. Bern: Forschungsstelle für Schulpädagogik und Fachdidaktik, Universität Bern.

Midgley, C., Maehr, M., Hruda, L., Anderman, E., Anderman, L., Freeman, K., Gheen, M., Kaplan, A., Kumar, R., Middleton, M.J., Nelson, J., Roeser, R., \& Urdan, T. (2000). Manual for the patterns of adaptive learning scales. Ann Arbor, MI: University of Michigan.

Maaz, K., Hausen, C., McElvany, N., \& Baumert, J. (2006). Stichwort: Übergänge im Bildungssystem - Theoretische Konzepte und ihre Anwendung in der empirischen Forschung beim Übergang in die Sekundarschule. Zeitschrift für Erziehungswissenschaft, 9(3), 299-327.

Moser, U., Oostlander, J., \& Tomasik, M. J. (2017). Soziale Ungleichheiten im Leistungszuwachs und bei Bildungsübergängen. In M. P. Neuenschwander \& C. Nägele (Hrsg.), Bildungsverläufe von der Einschulung in die Erwerbstätigkeit: Theoretische Ansätze - Befunde - Beispiele (S. 59-77). Wiesbaden: Springer VS.

Neuenschwander, M. P. \& Malti, T. (2009). Selektionsprozesse beim Übergang in die Sekundarstufe I und II. Zeitschrift für Erziehungswissenschaft, 12(2), 216-232.

Neuenschwander, M. P. (2016). Bildungsungleichheit am Beispiel der Leistungsentwicklung in Deutsch und Mathematik beim Übertritt in die Sekundarstufe I. In B. Ziegler (Hrsg.), (Un-)Gleichheiten in der Demokratie (S. 95-118). Zürich: Schulthess-Verlag.

Neuenschwander, M. P. (2017). Lern- und Leistungszielorientierung beim Übergang in die Sekundarstufe I: Längsschnittliche Befunde zur Bedeutung von Belastungen und Erziehungsverhalten von Eltern. Schweizerische Zeitschrift für Bildungswissenschaften, 39(2).

Neuenschwander, M. P., Rottermann, B., Niederbacher, E., Rösselet, S., \& Scheffler, L. (2014). Wirkungen der Selektion WiSel. Dokumentation der Leistungstests Mathematik und Deutsch der Welle 3 2013/2014. Solothurn: Zentrum Lernen und Sozialisation der PH FHNW.

Neuenschwander, M. P., Rösselet, S., Niederbacher, E., Rottermann, B., \& Scheffler, L. (2014). Wirkungen der Selektion WiSel. Dokumentation des Fragebogens für Schülerinnen und Schüler der Welle 3 2013/2014. Solothurn: Zentrum Lernen und Sozialisation der PH FHNW.

Pauli, C., Reusser, K., Waldis, M., \& Grob, U. (2003). „Erweiterte Lehr- und Lernformen“ im Mathematikunterricht der Deutschschweiz. Unterrichtswissenschaft, 31(4), 291-320.

Piaget, J. (1947). La psychologie de l'intelligence (dt. Psychologie der Intelligenz. Stuttgart: Klett, Trans. 8 ed.). Paris: Colin. 
Pintrich, P. R., Conley, A. M., \& Kempler, T. M. (2003). Current issues in achievement goal theory and research. International Journal of Educational Research, 39, 319-337. doi:10.1016/j. ijer.2004.06.002

Reusser, K., Pauli, C., \& Waldis, M. (Hrsg.). (2010). Unterrichtsgestaltung und Unterrichtsqualität. Münster: Waxmann.

Rösselet, S., \& Neuenschwander, M. P. (2017). Akzeptanz und Ablehnung von Schülerinnen und Schülern beim Übertritt in die Sekundarstufe I. In M. P. Neuenschwander \& C. Nägele (Eds.), Bildungsverläufe von der Einschulung in die Erwerbstätigkeit: Theoretische Ansät$z e-$ Befunde - Beispiele (pp. 103-121). Wiesbaden: VS Verlag für Sozialwissenschaften.

Sirsch, U. (2000). Probleme beim Schulwechsel. Münster: Waxmann.

Sternberg, R. J. (1997). The concept of intelligence and its role in lifelong learning and success. American Psychologist, 52(2), 1030-1038.

Van Ophuysen, S. (2008). Zur Veränderung der Schulfreude von Klasse 4- bis 7. Eine Längsschnittanalyse schulformspezifischer Effekte von Ferien und Grundschulübergang. Zeitschrift für Pädagogische Psychologie, 22, 293-306.

Whitley, J., Lupart, J. L. \& Beran, T. (2007). Differences in achievement between adolescents who remain in a K-8 school and those who transition to a junior high school. Canadian Journal of Education/Revue canadienne de l'éducation, 649-669.

Yen, W. M. \& Fitzpatrick, A. R. (2006). Item response theory. In R. L. Brennan (Ed.), Educational Measurement (pp. 111-154). Westport: Praeger Publisher.

Zimmer-Gembeck, M. J., Chipuer, H. M., Hanisch, M., Creed, P. A. \& McGregor, L. (2006). Relationships at school and stage-environment fit as resources for adolescent engagement and achievement. Journal of Adolescent Health, 29(6), 911-933. doi:10.1016/j.adolescence.2006.04.008

Open Access Dieses Kapitel wird unter der Creative Commons Namensnennung 4.0 International Lizenz (http://creativecommons.org/licenses/by/4.0/deed.de) veröffentlicht, welche die Nutzung, Vervielfältigung, Bearbeitung, Verbreitung und Wiedergabe in jeglichem Medium und Format erlaubt, sofern Sie den/die ursprünglichen Autor(en) und die Quelle ordnungsgemäß nennen, einen Link zur Creative Commons Lizenz beifügen und angeben, ob Änderungen vorgenommen wurden.

Die in diesem Kapitel enthaltenen Bilder und sonstiges Drittmaterial unterliegen ebenfalls der genannten Creative Commons Lizenz, sofern sich aus der Abbildungslegende nichts anderes ergibt. Sofern das betreffende Material nicht unter der genannten Creative Commons Lizenz steht und die betreffende Handlung nicht nach gesetzlichen Vorschriften erlaubt ist, ist für die oben aufgeführten Weiterverwendungen des Materials die Einwilligung des jeweiligen Rechteinhabers einzuholen.

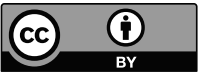


D

Übergänge in der Berufsbildung 


\section{Direkter oder verzögerter Ůbertritt? Das letzte Schuljahr zwischen Aspiration und Realisierung}

Albert Düggeli

\section{Zusammenfassung}

Jugendliche können ihren Übergang in die postobligatorische Ausbildung zeitlich mitgestalten. Während einige direkt und ohne Verzögerung in eine nachobligatorische Ausbildung eintreten, bevorzugen andere mehr Zeit für den Übergang. Die vorliegende Studie fokussiert auf Jugendliche, die trotz Direktaspiration verzögert übertreten. Es wird untersucht, inwiefern bei jenen, die einen direkten Übertritt anstreben, Merkmale des Leistungs- und Selbstsystems bzw. des Systems der sozialen Bezugnahme mit der tatsächlichen Umsetzung eines direkten oder verzögerten Anschlusses zusammenhängen. Aus einer laufenden Studie zur Bildungsmobilität (TIDES-Studie) liegen hierzu für den Kanton Basel-Stadt/CH entsprechende Daten vor. Analysiert wurden die Angaben von Jugendlichen aus den beiden Leistungszügen „Grundanforderungen“ bzw. „erweiterte Anforderungen“ der Sekundarstufe I ( $N=375)$. Es zeigte sich, dass bei Lernenden, die das Niveau mit erweiterten Anforderungen absolvieren, das kognitive Fähigkeitspotenzial und die am Ende der achten Klasse erreichte Deutschnote mit der Umsetzung einer Direktaspiration zusammenhängen. Alle anderen Merkmale, wie beispielsweise die Selbstwirksamkeit (Selbstsystem) oder die erfahrene Anerkennung von Eltern oder Lehrpersonen (soziales Bezugssystem), waren nicht mit der Realisierung einer Direktaspiration assoziiert. Bei der Abteilung mit Grundanforderungen zeigten sich keine substanziellen Zusammenhänge zwischen den untersuchten Merkmalen und der Umsetzung der Absicht, direkt in ein Anschlussangebot zu wechseln. Die Befunde werden mit Blick auf bildungssystemische und merkmalsspezifische Aspekte genauso diskutiert, wie sie auf der Folie der Berufswahlpraxis reflektiert werden. 


\section{Schlagworte}

Übergang in die berufliche Ausbildung; Berufswahl; Berufswunsch/Berufsrealisierung; Lokaler Ausbildungskontext

\section{Direkter oder verzögerter Übertritt? Das letzte Schuljahr zwischen Aspiration und Realisierung}

Gegen Ende der obligatorischen Schule planen die Heranwachsenden ihre nachobligatorische Ausbildung (Dreher \& Dreher, 1985; Häfeli, Neuenschwander \& Schumann, 2015; Stoll, Vanotti \& Schreiber, 2011; Düggeli \& Kinder, 2013). Dabei können sie nicht nur Ausbildungsinhalte oder Ausbildungsarten wählen, sondern auch den Übergang zeitlich mitgestalten. Möglich ist beispielsweise, dass sie einen Anschluss ohne zeitlichen Unterbruch anstreben, also direkt übertreten, oder den Übergang zeitlich dehnen und damit einen verzögerten Übergang bevorzugen. Direkte Pfade leiten nahtlos in weiterführende und in aller Regel zertifizierende Angebote über, verzögerte Wege führen über so genannte Übergangsausbildungen (Zwischenlösungen) ${ }^{1}$ und münden in Bildungsangebote, die gegenwärtig von 20 bis 25 Prozent der Lernenden gewählt werden (Landert \& Eberle, 2015; BFS. 2016). Diese Angebote scheinen hauptsächlich die Förderung und Unterstützung von Jugendlichen im Blick zu haben, also beispielsweise die Kompensation allfälliger schulischer oder allgemeiner Berufswahldefizite (vgl. z. B. Häfeli \& Schellenberg, 2009; BBG, 2002, Art. 12; Ratschinski \& Struck, 2012; Bayard Walpen, 2013). Damit zielen Übergangsausbildungen inhaltlich auf die Förderung positiver Entwicklungswege im schulbezogenen Leistungs- bzw. berufswahlbezogenen Selbstsystem sowie auf das berufswahlrelevante, soziale Bezugssystem von Heranwachsenden (vgl. z. B. Dedering, 2002; Stoll et al., 2011; Unterburger, 2012; Fend, 2003). In Abhängigkeit vom Entwicklungsstand bzw. von der Zielfokussierung kann es sich für einige vergleichsweise früh abzeichnen, verzögert einzusteigen. Für andere kristallisiert sich diese Möglichkeit vielleicht erst später heraus, allenfalls während des letzten Schuljahres und möglicherweise abweichend von ihrem ursprünglichen Wunsch, die berufliche Ausbildung direkt an die obligatorische Schulzeit anzuschliessen. Thematisch ist die vorliegende Untersuchung in diesem Feld situiert. Sie nimmt

1 Hierzu zählen beispielsweise so genannte 10. Schuljahre, Zentren für Brückenangebote oder Motivationssemester (vgl. Landert \& Eberle, 2015). Rechtlich sind diese Angebote im Artikel 12 des Berufsbildungsgesetzes abgestützt, BBG, 2002 (2016), Art. 12. 
Jugendliche in den Blick, die am Ende der achten Klasse eine direkte Anschlusslösung anstreben und diese ein Jahr später umsetzen bzw. fokussiert jene, die trotz Direktaspiration verzögert übertreten. Konkret wird die Frage untersucht, inwiefern bei Lernenden, die ein Jahr vor dem Übertritt einen direkten Anschlussweg anstreben, Merkmale des Leistungs-, des Selbstsystems bzw. des Systems der sozialen Bezugnahme mit der Realisierung eines direkten oder verzögerten Anschlusses zusammenhängen. Zunächst werden die Mittelwerte der untersuchten Merkmale zwischen den Direktumsetzenden und den verzögert Einsteigenden verglichen. Anschliessend zeigen regressionsanalytische Modellierungen Zusammenhänge zwischen Merkmalsausprägungen von Heranwachsenden und einer direkt oder indirekt realisierten Umsetzung einer Direktaspiration. Erwartet wird, dass die Befunde bestehendes Wissen zur berufswahlspezifischen Bedeutung des letzten Schuljahres erweitern und damit vorliegende längsschnittlich angelegte Übergangsstudien inhaltlich ausdifferenzieren (vgl. z. B. Bergman, Hupka-Brunner, Keller, Meyer \& Stalder, 2011; BFS. 2016).

\section{Direkte oder verzögerte Übergänge: Balancen zwischen Norm und Gestaltungsfreiheit}

Die Entscheidung, direkt oder verzögert in die nachobligatorische Ausbildung einzusteigen, kann unterschiedlich beurteilt werden. Gelten direkte Anschlüsse als anzustrebende Normalverläufe, erscheinen verzögerte Übergänge als davon abweichende und damit zu korrigierende Wege. Wird jedoch die Berufswahlfreiheit als Kriterium definiert, löst sich die Differenz zwischen Norm und Abweichung weitgehend auf. Berufswahlfreiheit identifiziert sämtliche, den Heranwachsenden offenstehenden Anschlussoptionen als gleichberechtigt und damit als frei wählbare Angebote, ohne eine normative Aufladung von direkten oder indirekten Anschlüssen. Zwischen dem erstgenannten, eher sozionormativ ausgerichteten Idealkonzept und dem zweitgenannten, eher die individuelle Handlungsfreiheit gewichtenden Freiheitskonzept, vermittelt der Fokus auf die Zielrealisierung als weitere Perspektive. Steht berufliche Gestaltungsfreiheit im Zentrum, und damit die Frage, inwiefern eine direkte oder zeitlich verzögerte Anschlusslösung dazu dient, das angestrebte Ausbildungsziel an einen möglichst auf dieses Ziel hinführenden Anfang zu binden, kann ein angestrebter und umgesetzter Direktanschluss der erforderliche und angemessene Weg sein. Genauso gut kann die Realisierung einer direkten Anschlussaspiration aber auch ein kritischer Weg sein, weil in die Direktumsetzung vielleicht grundlegende Aspekte der beruflichen Orientierung 
nicht ausreichend eingeflossen sind. In diesem Fall ist die Realisierung eines angestrebten Direkteinstiegs vielleicht ein zusätzlicher Schritt im Übergangsprozess, der bestehende Ideen nicht nur festigen, sondern auch destabilisieren kann. Unter dieser Bedingung ermöglichen reale Arbeitserfahrungen vielleicht erst zu erkennen, inwiefern die berufliche Eignung und das inhaltliche Interesse tatsächlich mit der zu erfüllenden Tätigkeit übereinstimmen. So betrachtet repräsentieren direkte Einstiege zeit- und realitätsnah geplante Umsetzungen einer veränderungsoffenen Ausbildungsabsicht.

Ähnlich wie die Realisierung einer Direktaspiration kann sich die Nichtrealisierung einer direkten Anschlussaspiration als produktiver Prozess oder als kritischer Verlauf erweisen. Produktiv - und damit gelingend - ist sie vielleicht dann, wenn sie hilft, eine angestrebte Ausbildung erreichen zu können, die im Moment noch nicht realisierbar ist, ein Jahr später aber mit höherer Wahrscheinlichkeit erreicht werden kann. In diesem Fall liegt allenfalls der Zeitpunkt der Entscheidung ungünstig, weil vielleicht zwischen der zeitlichen Taktung der Berufswahlphase, dem Berufswahlprozess selbst und den individuellen Entwicklungsverläufen Ungleichzeitigkeiten herrschen, deren Koordination wiederum Zeit erfordert (vgl. z. B. Düggeli, 2009). Unter dieser Perspektive gesteht ein verzögerter Einstieg der Berufswahl jene Zeiträume zu, die sie für ein möglichst gelingendes Ergebnis einfordert. Dies schliesst Optimierungsabsichten ein, also bspw. verzögerte Einstiege, die Heranwachsende dazu nutzen, um aus persönlichen Voraussetzungen bzw. vorhandenen Ausbildungsangeboten einen möglichst hohen Ausbildungswert zu generieren. Hierzu gehören Jugendliche, die gute Qualifikationen möglichst gewinnend spezifizieren und beispielsweise inhaltlich weiterentwickeln möchten. Es sind Ausbildungsoptimierende, die um den Wert der Ausbildung und um ihren eigenen Arbeitswert wissen und mit diesem auf dem Ausbildungsmarkt bewusst umgehen (vgl. auch Shell, 2015). Verzögerte Einstiege können sich aber auch als kritische Wege erweisen, weil sie beispielsweise inhaltlich nicht zielführend genutzt werden. In diesem Fall dienen sie Lernenden oft als Warteräume, in denen sie berufswahlbezogene Aktivitäten nur am Rande voranbringen, so dass ihre Berufswahlentwicklung nur wenig vorankommt. Dies kann mit mangelnder Selbstwirksamkeit oder mit der Überzeugung einhergehen, handlungsohnmächtig zu sein und bei den Lernenden zusehends das Gefühl von Inaktivität und Abkopplung auslösen (Düggeli, 2009; Grundmann, 2010). Diese unterschiedlichen Bewertungs- und Begründungszusammenhänge von direkten oder verzögerten Übertritten zeigen, dass die Wirkzusammenhänge entweder direkt oder verzögert einzusteigen, differenziert zu betrachten sind. Hierzu kann die Klärung der vorliegenden Forschungsfrage dienen, also zu erfahren, inwiefern bei Lernenden, die ein Jahr vor dem Übertritt einen direkten 
Anschlussweg anstreben, übertrittrelevante Merkmale mit der tatsächlichen Realisierung eines direkten Anschlusses zusammenhängen.

\section{$3 \quad$ Theoretische Einbettung und Modell}

Mit der Frage, direkt oder verzögert überzutreten, setzen sich Jugendliche in aller Regel handlungsplanend, handlungsumsetzend und ergebnisreflektierend auseinander (Düggeli \& Kinder, 2013). In diese Prozessfacetten fliessen mit Selbstwirksamkeit oder Leistungsfähigkeit Merkmale ein, die nicht nur die konkrete Anschlusswahl beeinflussen können, sondern auch als handlungsbezogene Aspekte in unterschiedlicher Weise in berufs- oder laufbahntheoretischen Modellierungen Eingang gefunden haben. So beispielsweise in einem von Herzog, Neuenschwander \& Wannak (2006) als Phasenkonzeption vorgestellten Konzept. Hinter den darin beschriebenen Phasen, also beispielsweise hinter der „Konkretisierung der Berufsorientierung" oder der "Suche nach einem Ausbildungsplatz“, stehen Prozessschritte des Handelns als zentrale Elemente der Berufswahl. Genauso zentral sind handlungsbezogene Prozessschritte aber auch in Modellen, die eine Selbstentwicklung durch die Berufswahl fokussieren, und zwar durch die Auseinandersetzung mit der Berufs- und Karrieregestaltung (Super, 1994; Savickas, 2005), oder durch den Aufbau einer Berufswahlkompetenz insgesamt (Ratschinski et al., 2012). Selbstbezogene Merkmale sind aber nicht alleinige Bezugsgrössen für die Frage, ob jemand eher einen direkten oder indirekten Anschluss wählt. Ebenso zentral sind fachliche Leistungen und damit Aspekte des Leistungssystems sowie Merkmale des sozialen Bezugssystems (vgl. z. B. Fend, 2003, vgl. Abb. 1). 


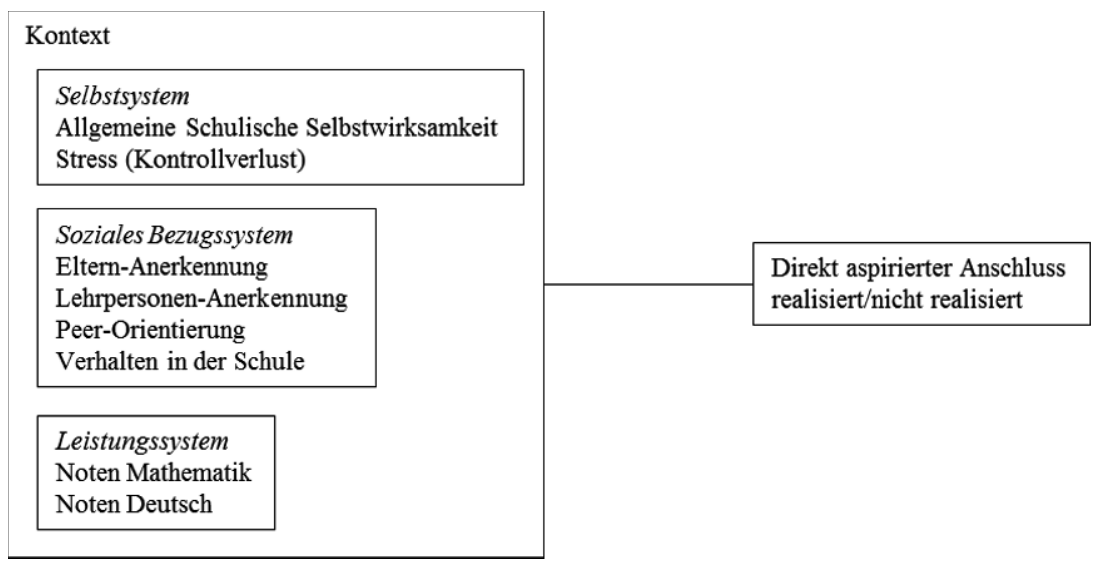

Abb. 1 Modellierung: Prozessdimensionen der Berufswahl und Anschlussrealisierung

Noten, im obigen Modell als Merkmale des schulischen Leistungssystems verortet, zählen zu den Kriterien mit grundlegender Bedeutung beim Übertritt in die postobligatorische Ausbildung (z. B. Moser, 2004). Reichen diese beispielsweise für eine angestrebte Ausbildung nicht aus, könnten sich Heranwachsende für eine Übergangsausbildung entscheiden und beabsichtigen, bestehende Lern- oder Wissenslücken aufzufüllen. Verzögerte Übertritte können aber auch durch selbstbezogene Aspekte verursacht werden, also beispielsweise durch eine gering ausgeprägte Selbstwirksamkeit oder durch ein hohes Stressempfinden, das Lernenden im Kontext der Berufswahl widerfahren kann, weil sie spezifische Kontrollverlusterfahrungen gemacht haben. Während eine geringe schulische Selbstwirksamkeit Lernenden erschweren kann, Handlungsanfänge zu initiieren oder Handlungsvollzüge mit Blick auf ein zu erreichendes Ziel durchzuhalten, könnte ein daraus resultierendes Stressempfinden Handlungsohnmacht hervorrufen (vgl. z. B. Flammer, 1990; Seligman, 1979). Ergänzend dazu erweitern Aspekte des sozialen Bezugssystems das Selbst- bzw. Leistungssystem. So spielt im Entwicklungskontext von Jugendlichen beispielsweise die Gleichaltrigengruppe eine zentrale Rolle. Mit Blick auf die Berufswahl ist diese Gruppe aber auch kritisch zu betrachten, weil sich ein hoher sozialer Partizipationsdruck auch ungünstig auf die Berufssuche auswirken könnte, beispielsweise weil Lernende dadurch davon abgehalten werden, ausreichend Zeit für ihre Berufswahlaktivitäten zu investieren. Soziale Erfahrungen machen Lernende aber auch, wenn sie beispielsweise von Lehrpersonen oder von den eigenen Eltern Anerkennung erfahren. Wenig Anerkennung kann als ungünstige Voraussetzung 
gelten, sich im zielorientierten Handeln positiv zu erfahren. Ganz im Gegensatz zu der Erfahrung, als Mensch grundsätzlich angenommen und damit anerkannt zu sein (vgl. z. B. Wischmann, 2010; Häfeli \& Schellenberg, 2009; Marty, Hirschi, Jungo, Jungo \& Zihlmann, 2011). Diese hier kurz zu den drei Bezugssystemen erwähnten Merkmale bilden den inhaltlichen Kern der vorliegenden Untersuchung.

\section{$4 \quad$ Untersuchung}

Zur Klärung der Frage, inwiefern bei Jugendlichen mit direkter Anschlussaspiration, Merkmale des Leistungs-, und des Selbstsystems bzw. des Systems der sozialen Bezugnahme mit der tatsächlichen Realisierung eines direkten Anschlusses zusammenhängen, wurden Daten aus der laufenden TIDES-Studie (Transitions in Different Educational Systems, 2012-2017) herangezogen. ${ }^{2}$ Diese Untersuchung analysiert die strukturelle Offenheit von Bildungssystemen bzw. die Nutzung von Durchlässigkeit beim Übergang in die postobligatorische Ausbildung in Deutschland (Baden-Württemberg) und der Schweiz (Basel-Stadt; Deutschfreiburg). Für Basel-Stadt stehen auf dieser Grundlage für die 8. und ein Jahr später für die 9. Klassenstufe Vollerhebungen zur Verfügung. Die vorliegende Untersuchung fokussiert auf die beiden niveaugestuften Schultypen (Weiterbildungsschule) der letzten beiden Schuljahre vor dem Übertritt. Das Niveau A (Abteilung A) der Weiterbildungsschule ist auf den Erwerb von Grundfertigkeiten ausgerichtet, das Niveau E (Abteilung E) strebt den Erwerb von erweiterten Fertigkeiten an. Da der Übertritt ins Gymnasium zum Erhebungszeitpunkt bereits erfolgt ist, steht für die Lernenden des Gymnasiums am Ende der neunten Klasse kein strukturell gegebener Übertritt an. Aus diesem Grund werden sie in den folgenden Analysen nicht berücksichtigt.

2 TIDES ist eine in Deutschland und der Schweiz durchgeführte Studie zur Analyse von struktureller Durchlässigkeit in unterschiedlichen Bildungssystemen (http://www. tides-study.ch/). Sie wird vom Schweizerischen Nationalfonds und der Deutschen Forschungsgemeinschaft unterstützt. 


\section{Stichprobe}

Herangezogen wurden die Daten aller Lernenden der Weiterbildungsschule (Abteilungen A bzw. E), die am Ende der achten Klasse einen direkten Übertritt anstrebten und von denen die gewählte Anschlusslösung (direkt oder verzögert) am Ende der neunten Klasse bekannt war (offizielle Schulabgangsstatistik ED Basel-Stadt, $N=375$ ). Von diesen Jugendlichen wurden soziostrukturelle und eine Reihe psychometrischer Merkmale am Ende der achten Klasse erfasst, so dass eine regressionsanalytische Modellierung mit Blick auf den definitiven Übertritt am Ende der 9. Klasse möglich wurde. Insgesamt haben 179 ihre Direktaspiration realisiert (47.7\%). 196 sind, entgegen ihrer Absicht, verzögert in die postobligatorische Ausbildung eingetreten (52.3\%). Bezogen auf die beiden Schultypen, ist dieses Bild nicht homogen. In der A-Abteilung (Grundanforderungen) realisieren 30 (25.2\%) die Direktaspiration und 89 (74.8\%) treten in ein Übergangsangebot ein. In der E-Abteilung (erweiterte Anforderungen) setzen mit 159 (56.8\%) im Vergleich zu 121 (43.2\%) mehr Jugendliche ihre Direktaspiration um. Wird zusätzlich das Geschlecht berücksichtigt, gibt es in der A-Abteilung 9 (30\%) Frauen bzw. 21 (70 \%) Männer, die einen aspirierten Direktanschluss realisieren. In dieser Gruppe treten, trotz direkter Aspiration, 40 (48.8\%) Frauen bzw. 42 (51.2\%) Männer verzögert in die nachobligatorische Ausbildung ein. In der E-Abteilung realisieren 86 (55.5\%) Frauen und 69 (44.5\%) Männer eine Direktaspiration, während in dieser Gruppe 64 (55.2\%) Frauen bzw. 52 (44.8\%) Männer verzögert übertreten, auch wenn sie einen direkten Anschluss angestrebt haben. Über beide Gruppen hinweg betrachtet, realisieren 96 (53.6\%) junge Frauen bzw. 83 (46.4\%) junge Männer eine Direktaspiration. 106 (54.1\%) heranwachsende Frauen setzen die Absicht, direkt überzutreten, nicht um, bei den Männern sind dies 90 (45.9\%).

\section{Gruppenvergleiche: Mittelwerte}

Der Blick auf die am Ende der achten Klasse erfassten Merkmale zeigt in der A-Abteilung keine nennenswerten Differenzen zwischen Jugendlichen mit direkter oder verzögerter Umsetzung der Direktaspiration. Auf der Basis der vorliegenden Daten kann also nicht davon ausgegangen werden, dass jene, die einen direkten Übergang anstreben, ein Jahr vor dem Übertritt beispielsweise über bessere Noten verfügen als ihre Mitlernenden mit nicht-realisierter Direktaspiration (vgl. Tab. 1). 
Tab. 1 Mittelwertvergleiche der untersuchten Merkmale zwischen den Gruppen mit realisierter bzw. nicht realisierter Direktanschlussaspiration; getrennt nach Abteilungen (Niveaustufen)

\begin{tabular}{|c|c|c|c|c|c|c|c|c|c|c|}
\hline \multirow[t]{3}{*}{ Variablen } & \multicolumn{5}{|c|}{ A-Abteilung } & \multicolumn{5}{|c|}{ E-Abteilung } \\
\hline & \multicolumn{2}{|c|}{$\begin{array}{l}\text { Aspiration } \\
\text { nicht reali- } \\
\text { siert }\end{array}$} & \multicolumn{2}{|c|}{$\begin{array}{l}\text { Aspiration } \\
\text { realisiert }\end{array}$} & & \multicolumn{2}{|c|}{$\begin{array}{l}\text { Aspiration } \\
\text { nicht reali- } \\
\text { siert }\end{array}$} & \multicolumn{2}{|c|}{$\begin{array}{l}\text { Aspiration } \\
\text { realisiert }\end{array}$} & \multirow[b]{2}{*}{ t-test } \\
\hline & M & SD & M & $\mathrm{SD}$ & t-test & M & SD & M & SD & \\
\hline \multicolumn{11}{|l|}{$\begin{array}{l}\text { Kontroll- } \\
\text { variablen }\end{array}$} \\
\hline $\begin{array}{l}\text { Sozioökono- } \\
\text { mischer Status } \\
\text { (HISEI) }\end{array}$ & 37.25 & 12.06 & 39.46 & 13.01 & -.85 & 43.27 & 15.70 & 47.51 & 16.39 & $-2.18^{\star *}$ \\
\hline $\begin{array}{l}\text { Kognitive } \\
\text { Fähigkeit } \\
\text { (KFT) }\end{array}$ & 14.42 & 4.66 & 14.68 & 5.37 & -.25 & 16.60 & 4.97 & 18.74 & 4.04 & $-3.86^{* *}$ \\
\hline \multicolumn{11}{|l|}{ Leistung } \\
\hline Note Deutsch & 4.23 & .70 & 4.29 & .61 & -.42 & 4.33 & .55 & 4.58 & .53 & $-3.8^{\star *}$ \\
\hline $\begin{array}{l}\text { Note Mathe- } \\
\text { matik }\end{array}$ & 3.86 & .93 & 4.14 & .95 & -1.40 & 4.09 & .73 & 4.43 & .78 & $-3.67^{\star *}$ \\
\hline \multicolumn{11}{|l|}{ Selbst } \\
\hline $\begin{array}{l}\text { Allg. schulische } \\
\text { Selbstwirksam- } \\
\text { keit }\end{array}$ & 2.88 & .46 & 2.95 & .41 & -.74 & 2.91 & .47 & 3.07 & .41 & $-3.08^{* *}$ \\
\hline $\begin{array}{l}\text { Stresswahrneh- } \\
\text { mung (Kon- } \\
\text { trollverlust) }\end{array}$ & 1.51 & 1.03 & 1.65 & 1.20 & -.62 & 1.86 & .95 & 1.65 & .99 & 1.76 \\
\hline \multicolumn{11}{|l|}{$\begin{array}{l}\text { Soziales } \\
\text { Bezugssystem }\end{array}$} \\
\hline $\begin{array}{l}\text { Anerkennung } \\
\text { der Eltern }\end{array}$ & 4.17 & .80 & 4.11 & .81 & .37 & 4.19 & .81 & 4.28 & .66 & -1.06 \\
\hline $\begin{array}{l}\text { Anerkennung } \\
\text { der Lehrper- } \\
\text { sonen }\end{array}$ & 4.00 & .98 & 3.93 & .76 & .36 & 3.87 & .91 & 3.87 & .76 & .03 \\
\hline $\begin{array}{l}\text { Extreme Peer- } \\
\text { Orientierung }\end{array}$ & 1.64 & .97 & 1.57 & 1.04 & .33 & 1.49 & 1.03 & 1.29 & .93 & 1.65 \\
\hline $\begin{array}{l}\text { Negatives } \\
\text { Verhalten } \\
\text { in der Schule }\end{array}$ & 3.25 & .60 & 3.22 & .55 & .23 & 3.34 & .54 & 3.40 & .51 & -.86 \\
\hline
\end{tabular}

Anmerkungen ${ }^{\star} \mathrm{p}<.05 .{ }^{* *} \mathrm{p}<.01$

HISEI= Indikator für den sozioökonomischen Status der Herkunftsfamilie; KFT = Kognitiver Fähigkeitstest 
Auch zeigt sich beispielsweise keine höhere Selbstwirksamkeit, bzw. berichten die Lernenden auch nicht über mehr erfahrene Anerkennung seitens ihrer Eltern oder ihrer Lehrpersonen. Gleichzeitig sind sie nicht weniger stark extremer Peerorientierung ausgesetzt bzw. finden sich zwischen diesen beiden Gruppen keine nennenswerten Unterschiede bezüglich ihres kognitiven Fähigkeitspotentials. Ebenso zeigen sich keine statistisch relevanten Unterschiede hinsichtlich ihres sozioökonomischen Status' ihrer Herkunftsfamilien oder mit Blick auf das Ausmass des auf Grund von Kontrollverlust erfahrenen Stresses. Und auch bezüglich negativer Verhaltensweisen in der Schule, unterscheiden sich die beiden Gruppen nicht bedeutsam voneinander.

Bei den Lernenden der E-Abteilung (erweiterte Anforderungen), präsentiert sich das Bild etwas anders. Hier verfügen die Direktaspirierenden mit realisierter Aspiration über ein höheres kognitives Fähigkeitspotential und über einen signifikant höheren sozioökonomischen Status ihrer Herkunftsfamilien. Lernende, die eine Direktaspiration realisieren, haben zudem ein Jahr vor dem Übertritt bessere Noten in Deutsch und Mathematik und weisen höhere Werte bezüglich der allgemeinen schulischen Selbstwirksamkeitserwartung aus. Die Mittelwerte der anderen Merkmale unterscheiden sich auch bei Lernenden dieser Abteilung nicht bedeutsam.

\section{$7 \quad$ Prädiktionsmodelle}

Hinsichtlich der Frage, inwiefern bei Lernenden, die einen direkten Anschluss anstreben, Zusammenhänge zwischen übertrittsrelevanten Merkmalen und der Umsetzung oder Nichtrealisierung ihrer direkten Anschlussaspiration bestehen, lassen sich bei der A- bzw. E- Abteilung unterschiedliche Muster erkennen (vgl. Tab. 2). Bei den Lernenden der Stufe mit Grundanforderungen (A-Abteilung) zeigen sich auf allen untersuchten Merkmalen keine statistisch abgesicherten Zusammenhänge (vgl. Modell A2). Selbst wenn in dieser Gruppe ausschliesslich das Geschlecht, der sozioökonomische Status und das kognitive Potential betrachtet werden, sind die Befunde statistisch nicht bedeutsam (vgl. Modell A1). Bezogen auf die E-Abteilung, also auf die Stufe mit erweiterten Anforderungen, erweist sich das kognitive Potential und die Deutschnote als bedeutsam dafür, die Absicht umzusetzen, direkt in eine Ausbildung auf der Sekundarstufe II überzutreten (vgl. Modell E2). Die kognitive Fähigkeit ist auch dann bedeutsam, wenn sie separat mit dem sozioökonomischen Status der Familie und dem Geschlecht betrachtet wird (vgl. Modell E1). Für Lernende, die den Übergang aus der Stufe mit erweiterten Anforderungen heraus gestalten, tragen also das ein Jahr vor dem Übertritt erfasste kognitive Leistungspotential sowie 
die Deutschnote dazu bei, einen erwünschten, direkten Anschluss zu realisieren. Bei Lernenden, welche die Schulstufe mit Grundanforderungen absolvieren, zeigt sich dieser Effekt nicht. Bei ihnen erweist sich auch kein anderes der erfassten Merkmale als bedeutsam, einen aspirierten Direktanschluss zu realisieren.

Tab. 2 Regressionsanalytische Zusammenhänge zwischen den erfassten Merkmalen und der Realisierung oder Verzögerung einer direkten Übertrittaspiration

\begin{tabular}{|c|c|c|c|c|c|c|c|c|c|c|}
\hline \multirow[b]{3}{*}{ Variablen } & \multicolumn{4}{|c|}{ A-Abteilung } & \multicolumn{6}{|c|}{ E-Abteilung } \\
\hline & \multicolumn{2}{|c|}{ Modell A1 } & \multicolumn{2}{|c|}{ Modell A2 } & \multicolumn{3}{|c|}{ Modell E1 } & \multicolumn{3}{|c|}{ Modell E2 } \\
\hline & B & SE B & B & SE B & B & SE B & & B & SE B & \\
\hline \multicolumn{11}{|l|}{ Kontrollvariablen } \\
\hline Geschlecht & .78 & .46 & .75 & .52 & -.08 & .26 & & -.03 & .30 & \\
\hline $\begin{array}{l}\text { Sozioökonomischer } \\
\text { Status (HISEI) }\end{array}$ & .02 & .02 & .01 & .02 & .01 & .00 & & .01 & .01 & \\
\hline $\begin{array}{l}\text { Kognitive Fähigkeit } \\
\text { (KFT) }\end{array}$ & .02 & .04 & -.00 & .05 & .10 & .03 & * & .08 & .03 & * \\
\hline \multicolumn{11}{|l|}{ Leistung } \\
\hline Note Deutsch & & & -.20 & .41 & & & & .78 & .27 & * \\
\hline Note Mathematik & & & .33 & .27 & & & & .32 & .20 & \\
\hline \multicolumn{11}{|l|}{ Selbst } \\
\hline $\begin{array}{l}\text { Allgemeine schu- } \\
\text { lische Selbstwirk- } \\
\text { samkeit }\end{array}$ & & & .06 & 65 & & & & .70 & .37 & \\
\hline $\begin{array}{l}\text { Stresswahrneh- } \\
\text { mung (Kontroll- } \\
\text { verlust) }\end{array}$ & & & .19 & .22 & & & & -.10 & .15 & \\
\hline \multicolumn{11}{|l|}{$\begin{array}{l}\text { Soziales } \\
\text { Bezugssystem }\end{array}$} \\
\hline $\begin{array}{l}\text { Anerkennung } \\
\text { der Eltern }\end{array}$ & & & -.07 & .31 & & & & -.07 & .21 & \\
\hline $\begin{array}{l}\text { Anerkennung der } \\
\text { Lehrpersonen }\end{array}$ & & & -.05 & .28 & & & & -.24 & .18 & \\
\hline $\begin{array}{l}\text { Extreme Peer- } \\
\text { Orientierung }\end{array}$ & & & -.15 & .28 & & & & -.21 & .15 & \\
\hline $\begin{array}{l}\text { Negatives Verhalten } \\
\text { in der Schule }\end{array}$ & & & -.20 & .45 & & & & -.06 & .30 & \\
\hline Pseudo $\mathrm{R}^{2}$ & & 05 & 0 & 9 & & & & .2 & & \\
\hline
\end{tabular}

Anmerkungen ${ }^{*} p<.05$

Referenzkategorie $=$ direkter Übertritt

HISEI= Indikator für den sozioökonomischen Status der Herkunftsfamilie; KFT = Kognitiver Fähigkeitstest 


\section{$8 \quad$ Interpretation}

Besonders interessant ist, dass sich bei Direktaspirierenden des Niveaus mit Grundanforderungen (A-Abteilung) kein statistisch abgesicherter Zusammenhang hinsichtlich der Leistungsaspekte (Noten) zeigt. Leistungsdefizite, die sich beispielsweise in ungenügenden Noten ausdrücken, werden oft als Grund für zeitlich verzögerte Einstiege genannt (vgl. Abschnitt 1). Vorliegend konnte dieser Effekt jedoch zumindest für diese Abteilung nicht gefunden werden. Zieht man zusätzlich in Betracht, dass in der A-Abteilung das niedrigste Anforderungsniveau besteht, könnten die Lernenden möglicherweise einer Art „negativem Kumulationseffekt“ ausgesetzt sein, der sich formal aus dem besuchten Schulniveau und inhaltlich aus den in diesem Anforderungsniveau erbrachten Leistungsmass (Noten) zusammensetzt (Solga, 2002). Vielleicht muss mit Blick auf die Noten aber auch darauf hingewiesen werden, dass die ein Semester später erhaltenen Noten ebenfalls für den Übergangsprozess ausschlaggebend sein könnten, also Leistungsmasse, die hier noch keinen Eingang gefunden haben. Und ebenso gilt es im Kontext des Leistungsaspekts von Lernenden dieser Abteilung darüber nachzudenken, inwiefern die Aspirationen auf der Folie eines in Basel-Stadt differenziert ausgebauten Übergangssystems zu lesen sind. So steht den Lernenden am Übergang eine Vielzahl von Ausbildungsmöglichkeiten dieses Systems zur Verfügung. Sie können also vielfältige Angebote wählen, um beispielsweise allfällige Defizite aufarbeiten oder angestrebte Entwicklungswege initiieren zu können.

Bei den Lernenden der E-Abteilung hingegen scheinen das kognitive Fähigkeitspotential und die Deutschnote bereits ein Jahr vor dem Übertritt die Umsetzung einer direkten Aspiration zu begünstigen. Analog zum bereits erwähnten „negativen Kumulationseffekt" für Lernende der A-Abteilung könnte hier umgekehrt von einem „positiven Kumulationseffekt“ mit entsprechender Signalwirkung gesprochen werden (vgl. hierzu auch Keller \& Moser, 2013). Weitere hier analysierte Merkmale fallen aber auch bei den Lernenden dieser Abteilung nicht ins Gewicht. Weder die allgemeine schulische Selbstwirksamkeitserwartung noch beispielsweise die von Eltern oder Lehrpersonen erfahrene Anerkennung oder auch der gegebenenfalls durch die Wahrnehmung von Kontrollverlust erfahrene Stress beeinflussen eine direkte oder indirekte Umsetzung einer Direktaspiration systematisch.

Vergleicht man nun die Befunde zwischen den beiden Abteilungen, zeigt sich relativ deutlich, dass die untersuchten Merkmale zur Erklärung einer direkten oder verzögerten Realisierung bei direkter Anschlussaspiration insgesamt nur begrenzt bedeutsam sind. Ebenfalls scheint die auf der Sekundarstufe I besuchte Abteilung im Kontext von direkt oder verzögert realisierten Anschlüssen bei einer Direktaspiration nicht unterschätzt werden zu dürfen. Somit lässt sich darüber 
nachdenken, ob eine Art Abkopplung und/oder Stigmatisierung der Lernenden dieses Schultyps generell besteht, die sich bei Heranwachsenden der Abteilung mit Grundanforderungen zusätzlich verschärft. Und nicht zuletzt machen die Ergebnisse deutlich, dass viele der Merkmale, von denen im Allgemeinen davon ausgegangen wird, dass sie wichtig für eine gelingende Berufswahl und damit übertrittsrelevant sind, unter diesem analytischen Blick nur als wenig bedeutsam erweisen. Welche anderen Faktoren oder allfälligen Interaktionseffekte zwischen ihnen eine Rolle spielen könnten oder ob auch situationsbezogene Aspekte mitmodelliert werden, ist offen, bleibt allenfalls ein thematischer Aspekt der nachfolgenden Diskussion.

\section{Diskussion}

Die vorliegenden Ergebnisse werden abschliessend unter einer forschungs- bzw. praxisorientierten Perspektive diskutiert. Dies erfolgt zunächst mit Blick auf eine föderale Bildungssteuerung, wie sie für die Schweiz grundlegend ist. Anschliessend erfolgt ein Fokus auf konkrete Handlungs- und Erfahrungszusammenhänge, so dass die handelnden Individuen besonders betrachtet werden. Eine dritte Perspektive versucht schliesslich, die Befunde hinsichtlich der Handlungspraxis von unterstützenden Personen zu verorten, beispielsweise von Lehr- oder Berufsberatungspersonen.

Die bildungssystemische Einordnung der Ergebnisse hat primär im Kontext des Referenzsystems zu erfolgen, in dem die Daten erhoben wurden. Es gilt also, die Wahl von Anschlussangeboten im Rahmen kantonaler Bildungssysteme zu betrachten. Wird eine systemübergreifende Einordnung angestrebt, sind auch solche Kriterien zu definieren. Inwiefern dies im schweizerischen Bildungskontext jedoch gelingt, ist eine offene Frage, die aber, je nach Erkenntnisabsicht von weiterführenden Untersuchungen, neu zu diskutieren ist. Ebenso gilt unter einem systemischen Fokus, dass Lernende bei diesem Übergang von einem obligatorischen und kantonal implementierten Schulsystem in ein nachobligatorisches Ausbildungssystem wechseln. Dies eröffnet ihnen beispielsweise die Möglichkeit, nicht nur die Ausbildungsart zu wählen, sondern auch den Ausbildungsort. Dieser kann ohne weiteres ausserhalb des Kantons liegen, in dem Lernende ihre obligatorische Schulzeit absolviert haben. Somit lassen sich die vorliegenden Befunde auch auf der Folie eines Zusammenspiels von obligatorischen und an kantonale Steuerungen gebundene Ausbildungen und einem frei spielenden, postobligatorischen Ausbildungssystem reflektieren. Inwiefern also die Wahl oder die Vergabe einer Lehrstelle mit dem während der obligatorischen Schulzeit besuchten Schulsystem zusammenhängt, 
ist eine wichtige Frage mit Blick auf direkte oder indirekte Übertritte. Während sich also systematisch Aspekte für die einen als hilfreich erweisen können, eine Direktaspiration zu realisieren, könnte für andere die Umsetzung eines direkten Anschlusswunsches deshalb erschwert sein, weil sie die obligatorische Schulzeit in einem anderen Bildungssystem absolviert haben. Vielleicht ist es unter dieser Bedingung angezeigt, eine Ausbildungsregion als Ganzes in den Blick zu nehmen, und zwar auch auf der Volksschulstufe.

An diesen Gedanken schliesst der zweite Diskussionspunkt an. Er stellt die untersuchten Merkmale mit spezifischem Blick auf kantonale Bildungssysteme ins Zentrum. Reflektiert wird die Frage, inwiefern die untersuchten Merkmale in allen Schulsystemen ähnlich relevant sind, bzw. inwiefern nicht systemspezifische Gewichtungen angezeigt wären. Vielleicht sind für Lernende aus Basel-Stadt andere Merkmale bedeutsam, damit sie eine Direktaspiration realisieren können, als beispielsweise für Lernende eines anderen Kantons. Und denkbar ist auf Grund der eher geringen Effekte zudem, dass die erfassten Merkmale den Bereich so genannter übergangsrelevanter Merkmale zu wenig ausreichend abdecken. Gerade mit Blick auf den konkreten Handlungsbezug und damit hinsichtlich konkreter Berufswahlerfahrungen werden hier eher wenig Aspekte berücksichtigt. Übergangsrelevante Merkmale sind also auch in ihrer situationsbezogenen und damit kontextuellen Bedeutung zu erfassen, so wie sie auch in ihrer phasenbezogenen Wichtigkeit einzubeziehen sind. So belegen beispielsweise schulische Noten in aller Regel über ein Semester hinweg, allenfalls sogar darüber hinausgehend, die erbrachten Leistungen. Das Stressempfinden hingegen ist vielleicht eher an konkrete Situationen gebunden, auch wenn es zweifelsohne Belastungen durch permanentes Stressempfinden gibt. Solche konkreten Handlungserfahrungen in ihrem Zeitbezug sind im Kontext der Realisierung eines direkten oder verzögerten Übergangs bei einer Direktaspiration möglichst einzubeziehen.

Abschliessend und noch etwas stärker auf die Berufswahlpraxis bezogen, werden die vorliegenden Befunde bzw. die dazu vorgenommenen Interpretations- und Diskussionsansätze bezüglich der Unterstützung von Jugendlichen im Berufswahlprozess betrachtet. Wenn die Berufswahlbegleitung auf eine möglichst optimale Einmündung in die Berufsausbildung zielt, kann dies bedeuten, gemeinsam mit Heranwachsenden den Zeitpunkt zu identifizieren, wann es beispielsweise sinnvoll ist, einem aspirierten Direktanschluss einen verzögerten Einstieg vorzuziehen, möglichst unter gleichzeitiger Kompensation von allfällig bestehenden Defiziten. Wenn aber davon auszugehen ist, dass Defizite im Leistungssystem nur wenig wahrscheinlich aufgeholt werden können, weil der Abstand zwischen Anforderung und individuellen Möglichkeiten als sehr gross erscheint, ist vielleicht ein Abweichen von einem angestrebten Direktanschluss nur wenig erfolgswahrscheinlich und 
damit nicht zielführend. Vielmehr könnte sich dann ein Wechsel ins Übergangssystem als Aufschub erweisen, der ein Jahr später zu der ernüchternden Einsicht führt, dass das angestrebte Ziel doch nicht realisiert werden kann. Solche Verläufe könnten psychische Belastungen auslösen oder verstärken, so dass vielleicht ein Direktanschluss der angemessenere und von weniger Risiken behaftete Weg wäre. Und auch bezüglich der hier untersuchten inhaltlichen Aspekte des Leistungs- bzw. Selbstbereichs bzw. des sozialen Bezugssystems, muss mit Blick auf die strukturell gegebenen Möglichkeiten differenziert werden. Während beispielsweise Anerkennungsfacetten situationsübergreifend und als Grundlage zwischenmenschlicher Begegnungen schlechthin gelebt werden, müssen vielleicht Leistungsmerkmale oder Wirksamkeitsaspekte stärker im Verhältnis von Aspiration, Handlungserfahrungen und real möglicher Umsetzungswahrscheinlichkeit betrachtet werden. Je nachdem deutet dieses Verhältnis darauf hin, eine direkte Anschlussaspiration durchzuhalten. Genauso gut kann dieses Verhältnis aber auch darauf verweisen, den geplanten Berufswahlweg zeitlich zu dehnen und ein Übergangsangebot zu wählen. Dabei ist jedoch wichtig zu sehen, dass ein Entscheid für mehr Zeit nicht gleichzeitig heisst, sich weniger aktiv im Berufswahlprozess zu engagieren. Auch wenn es angemessen ist, einen verzögerten Einstieg zu realisieren, gibt es beispielsweise wenig Gründe dafür, keine Schnupperlehren zu absolvieren oder keine Bewerbungstrainings oder individuelle Fähigkeitsanalysen zu machen. Ohne situatives Handeln brechen für Heranwachsende oft grundlegende Möglichkeiten weg, zentrale Bereiche ihrer Berufswahl- oder Laufbahngestaltungskompetenz zu entwickeln. 


\section{Literatur}

BBG Berufsbildungsgesetz (2002). Stand 1. Januar 2016, Art. 12.

Bayard Walpen, S. (2013). Obligatorischer Schulabschluss. Wie weiter? Zur Bedeutung von Kompetenzeinschätzungen für den Übertritt in eine nachobligatorische Ausbildung. Zürich: Seismo.

Bergman, M. M., Hupka-Brunner, S., Keller, A., Meyer, T., \& Stalder, B. E. (2011). Transitionen im Jugendalter: Ergebnisse der Schweizer Längsschnittstudie TREE. Zürich: Seismo.

BFS (2016). Längsschnittanalysen im Bildungsbereich. Der Übergang am Ende der obligatorischen Schule. Ausgabe 2016. Neuchâtel: BFS.

Dedering, H. (2002). Entwicklung der schulischen Berufsorientierung in der Bundesrepublik Deutschland. In J. Schudy (Hrsg.), Berufsorientierung in der Schule. Grundlagen und Praxisbeispiele (S. 17-31). Bad Heilbrunn: Julius Klinkhardt.

Dreher, E., \& Dreher, M. (1985). 'Entwicklungsaufgabe' - theoretisches Konzept und Forschungsprogramm. In R. Oerter (Hrsg.), Lebensbewältigung im Jugendalter (S. 30-61). Weinheim: Edition Psychologie.

Düggeli, A., \& Kinder, K. (2013). Ich trau mich schon und kann das auch: Jugendliche bewältigen Berufswahlbesorgnisse mit ihren Lehrpersonen. In T. Brüggemann \& S. Rahn (Hrsg.), Berufsorientierung (S.211-219). Münster: Waxmann.

Düggeli, A. (2009). Ressourcenförderung im Berufswahlunterricht / Interventionsstudie mit Lernenden der Sekundarstufe I, Niveau Grundanforderungen. Münster, München, Berlin [u.a. ]: Waxmann.

Fend, H. (2003). Entwicklungspsychologie des Jugendalters. Weinheim und Basel: Beltz Verlag.

Flammer, A. (1990). Erfahrung der eigenen Wirksamkeit / Einführung in die Psychologie der Kontrollmeinung. Berlin: Huber.

Grundmann, M. (2010). Handlungsbefähigung - eine sozialisationstheoretische Perspektive. In H.-U. Otto \& H. Ziegler (Hrsg.), Capabilities - Handlungsbefähigung und Verwirklichungschancen in der Erziehungswissenschaft (S. 131-141). Münster: Waxmann.

Häfeli, K., \& Schellenberg, C. (2009). Erfolgsfaktoren in der Berufsbildung bei gefährdeten Jugendlichen. Bern: Schweizerische Konferenz der kantonalen Erziehungsdirektoren (EDK).

Häfeli, K., Neuenschwander, M., \& Schumann, S. (Hrsg.). (2015). Berufliche Passagen im Lebenslauf. Berufsbildungs- und Transitionsforschung in der Schweiz. Wiesbaden: Springer VS.

Herzog, W., Neuenschwander, M. P., \& Wannack, E. (2006). Berufswahlprozess. Bern, Stuttgart, Wien: Haupt Verlag.

Keller, F., \& Moser, U. (2013). Schullaufbahnen und Bildungserfolg. Auswirkungen von Schullaufbahnen und Schulsystem auf den Übertritt ins Berufsleben. Zürich/Chur: Rüegger Verlag.

Landert, C. \& Eberli, D. (2015). Bestandsaufnahme der Zwischenlösungen an der Nahtstelle I. Bericht. Zürich: Sozialforschung, Evaluation, Konzepte.

Marty, R., Hirschi, A., Jungo, D., Jungo, M., \& Zihlmann, R. (2011). Berufswahlfreiheit. Ein Modell im Spannungsfeld zwischen Individuum und Gesellschaft. Bern: SDBB Verlag.

Moser, U. (2004). Jugendliche zwischen Schule und Berufsbildung. Eine Evaluation bei Schweizer Grossunternehmen unter Berücksichtigung des internationalen Schulleistungsvergleichs PISA. Bern: hep Verlag AG.

Ratschinski, G., \& Struck, P. (2012). Die entwicklungspsychologischen Grundlagen der Ausbildungsreife. In A. Bojanowski \& M. Eckert (Hrsg.), Black Box. Übergangssystem (S.171-182). Münster, New York: Waxmann. 
Savickas, M. L. (2005). The Theory and Practice of Career Construction. In S. D. Brown \& R. W. Lent (Eds.), Career Development and Counseling: Putting Theory and Research to Work (pp. 42-70). Hoboken New Jersey: Wiley.

Seligman, M. (1979). Erlernte Hilflosigkeit. München: Urban und Schwarzenberg.

Shell (Hrsg.) (2015). Jugend 2015: 17. Shell Jugendstudie. Frankfurt a. Main: Fisher Verlag.

Solga, H. (2002). Stigmatization by Negative Selection'. Explaining Less-Educated People's Decreasing Employment Opportunities. European Sociological Review, 18(2), 159-178.

Stoll, F., Vannotti, M., \& Schreiber, M. (2011). Einstieg in die Berufswelt. Rahmenbedingungen und Voraussetzungen einer gelingenden Berufswahl - eine empirische Studie. Glarus/ Chur: Rüegger Verlag.

Super, D. E. (1994). Der Lebenszeit-, Lebensraumansatz der Laufbahnentwicklung. In D. Brown \& L. Brooks (Hrsg.), Karriere- Entwicklung (S.211-280). Stuttgart: Klett-Cotta.

Unterburger, S. (2012). Berufswahlreife. Übersicht zur Theorienlage und Entwicklung eines Messinstruments. Saarbrücken: Akademiker Verlag.

Wischmann, A. (2010). Adoleszenz-Bildung - Anerkennung. Adoleszente Bildungsprozesse im Kontext sozialer Benachteiligung. Wiesbaden. VS Verlag.

Open Access Dieses Kapitel wird unter der Creative Commons Namensnennung 4.0 International Lizenz (http://creativecommons.org/licenses/by/4.0/deed.de) veröffentlicht, welche die Nutzung, Vervielfältigung, Bearbeitung, Verbreitung und Wiedergabe in jeglichem Medium und Format erlaubt, sofern Sie den/die ursprünglichen Autor(en) und die Quelle ordnungsgemäß nennen, einen Link zur Creative Commons Lizenz beifügen und angeben, ob Änderungen vorgenommen wurden.

Die in diesem Kapitel enthaltenen Bilder und sonstiges Drittmaterial unterliegen ebenfalls der genannten Creative Commons Lizenz, sofern sich aus der Abbildungslegende nichts anderes ergibt. Sofern das betreffende Material nicht unter der genannten Creative Commons Lizenz steht und die betreffende Handlung nicht nach gesetzlichen Vorschriften erlaubt ist, ist für die oben aufgeführten Weiterverwendungen des Materials die Einwilligung des jeweiligen Rechteinhabers einzuholen.

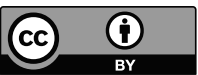




\section{Passung zum Beruf und die Wahl einer Aus- oder Weiterbildung}

Christof Nägele, Simone Frey und Markus P. Neuenschwander

\section{Zusammenfassung}

Eine hohe Passung zum Beruf wird als ein wesentlicher Faktor für eine hohe Zufriedenheit und Verbundenheit mit dem Beruf angesehen. Eine sich verändernde Passung sollte deshalb auch Laufbahnentscheidungen erklären. Dabei kann die Planung einer Aus- oder Weiterbildung dazu genutzt werden, die (veränderte) Passung zum Beruf zu erhöhen. Deshalb kann angenommen werden, dass Personen mit einer geringen Passung zum Beruf eher eine Ausoder Weiterbildung planen. Die Passung einer Person zu ihrem Beruf und zur Weiterbildung kann einerseits als wahrgenommene Passung sowie als auf dem Person-Umwelt-Modell von Holland basierende Kongruenz dargestellt werden.

Es kann gezeigt werden, dass die wahrgenommene Passung sowohl die Zufriedenheit und Verbundenheit mit dem Beruf, wie auch die Planung einer Aus- oder Weiterbildung vorhersagt. Die Kongruenz Person-Beruf vermag dies nicht. Personen, die eine Aus- oder Weiterbildung planen, tun dies in der Regel so, dass diese besser zu ihnen passt als der aktuelle Beruf. Dies gilt jedoch nur für Personen mit einer tiefen Passung zu ihrem Beruf. Die Ergebnisse legen eine wichtige Rolle der wahrgenommenen Passung in der Bewertung und Steuerung der beruflichen Laufbahn nahe, stellen aber die Nützlichkeit einer auf Interessen beruhenden Bestimmung der Kongruenz in Frage.

\section{Schlagworte}

Laufbahn, Berufsbildung, Weiterbildung, Zufriedenheit, Verbundenheit 


\section{$1 \quad$ Passung zum Beruf und die Wahl einer Aus- oder Weiterbildung}

Eine kontinuierliche Aus- und Weiterbildung ist wichtig, und die Frage stellt sich bereits zu Beginn der beruflichen Laufbahn nach Abschluss einer beruflichen Grundbildung. Die Entscheidung, eine Aus- oder Weiterbildung zu planen oder zu besuchen, wird durch motivationale Faktoren (Neuenschwander, Nägele, \& Frey, n.d.) oder die Wahrnehmung von Chancen und Hindernissen, zum Beispiel in Zusammenhang mit der Vereinbarkeit von Familie und Arbeit (Frey, Neuenschwander \& Nägele, 2016) beeinflusst. In diesem Kapitel wird den Fragen nachgegangen, welchen Einfluss eine hohe Passung zum Beruf auf die Zufriedenheit und Verbundenheit mit der Arbeit hat und wie die Passung zum aktuellen Beruf die Planung einer Aus- oder Weiterbildung beeinflusst.

Eine hohe subjektive Passung zum Beruf ist nicht nur ein wichtiger Prädiktor für einen positiven Einstieg in eine neue Organisation (Nägele \& Neuenschwander, 2015), sondern auch eine Voraussetzung für die Entwicklung von Zufriedenheit und Verbundenheit mit dem Beruf (Holland, 1997; Super, 1980). Verändert sich die Passung zum Beruf, kann dies Veränderungen und Transitionen auslösen (Kristof-Brown, Zimmerman, \& Johnson, 2005). Wird die Passung geringer, besteht eine Möglichkeit darin, diese durch proaktives Verhalten (Grant \& Ashford, 2008) oder Job-crafting (Tims, Bakker, \& Derks, 2012) wieder zu verbessern. Wenn die eigenen Bedürfnisse und Interessen im aktuellen Beruf jedoch nicht mehr erfüllt werden können und somit kein Need-satisfaction -fit erzielt wird (Kristof-Brown et al., 2005), kann eine weitere Aus- oder Weiterbildung eine Option sein, um sich für einen anderen, passenderen Beruf zu qualifizieren. Durch die Wahl einer weiteren Aus- oder Weiterbildung passt eine Person ihre Umwelt so an, dass sie ihr optimale Entwicklungsmöglichkeiten bietet, im Sinne der Stage-environement-fit-Theorien (Eccles et al., 1993). Als aktive Gestalter ihrer eigenen Entwicklung steuern Personen so ihre berufliche Entwicklung (Savickas, 2012).

Zusammenfassend lässt sich festhalten, dass die Passung einer Person mit ihrem Beruf wichtig für die Beurteilung der aktuellen beruflichen Situation und auch für die Planung oder den Besuch einer Aus- oder Weiterbildung ist. Eine geringe Passung kann sich negativ auf die Befindlichkeit auswirken und Bildungsaktivitäten auslösen. Dies mit dem Ziel, die Passung (wieder) zu erhöhen. 


\subsection{Wahrgenommene Passung und Kongruenz Person-Beruf}

Passung ist als Übereinstimmung von Eigenschaften der Person mit Eigenschaften ihrer Umwelt definiert (Kristof, 1996). Die Passung einer Person zu ihrem Beruf kann anhand subjektiver oder objektiver Massen dargestellt werden (Judge, 2007).

Die wahrgenommene Passung entsteht aufgrund eines Vergleichs beruflicher und individueller Werte (Cable \& Judge, 1996). Eine hohe wahrgenommene Passung von Person und Beruf führt zu einer höheren beruflichen Zufriedenheit und Einschätzung des beruflichen Erfolgs. Bei einer guten Passung entwickelt sich die berufliche Laufbahn positiver (Hansen \& Lee, 2007; Neuenschwander, Gerber, Frank, \& Rottermann, 2012). Personen mit einer hohen Passung verbleiben eher in einer Organisation, sind leichter zu motivieren und erbringen bessere Leistungen (Kristof, 1996; Taris, Feij, \& Capel, 2006). Eine hohe Passung ist insgesamt ein guter Indikator für eine erfolgreich verlaufene Anpassung an den Beruf und die Organisation (Bauer \& Erdogan, 2011). Personen mit einer tiefen Passung haben hingegen ein erhöhtes Fluktuationsrisiko, sowohl in der beruflichen Grundbildung als auch in der Erwerbsarbeit (Stalder \& Schmid, 2016). Die wahrgenommene Passung verändert sich aufgrund neuer Erfahrungen (Nägele \& Neuenschwander, 2015).

Ein teilobjektives Mass der Passung ist die Kongruenz. Nach dem Person-Umwelt-Modell von Holland (1997) und Parsons (1909) können sowohl Personen als auch Berufe Typen zugeordnet werden. Der Personentyp beruht auf der Darstellung der Interessen einer Person, unterschiedliche berufliche Tätigkeiten ausführen zu wollen. Interessen werden als Ausdruck individueller Vorlieben verstanden. Die Typisierung der Berufe erfolgt anhand objektiver Kriterien durch Expertinnen und Experten (Jörin Fux, Stoll, Bergmann, Eder, \& Hell, 2012), in welchem Ausmass diese Tätigkeiten in dem entsprechenden Beruf vorkommen. Die Kongruenz ist der Grad der Übereinstimmung des Personen- und Umwelttyps (Jörin Fux et al., 2012). Zur Bestimmung der Kongruenz wurde der C-Index nach Brown (1994) berechnet, der die Anordnung der drei am stärksten ausgeprägten Interessendimensionen gemäss dem Hexagonmodell von Holland (1997) berücksichtigt (Jörin Fux, 2005).

\subsection{Zufriedenheit und Verbundenheit}

Zwei wichtige Ergebnisse einer erfolgreichen Anpassung an den Beruf sind die Zufriedenheit und die Verbundenheit mit dem Beruf (Bauer, Bodner, Erdogan, Truxillo, \& Tucker, 2007; Kammeyer-Mueller \& Wanberg, 2003). Zahlreiche Studien belegen, dass eine hohe Zufriedenheit als Ergebnis eines erfolgreichen Anpassungs- 
prozesses und als Voraussetzung für eine positive weitere Entwicklung wichtig ist (Keller \& Semmer, 2013).

Die Verbundenheit mit dem Beruf (Commitment) ist eine affektive Reaktion auf die aktuelle Situation (Cohen, 2007), die eng mit der Entwicklung einer beruflichen Identität verbunden ist (Stalder \& Nägele, 2015). Personen mit einer hohen Verbundenheit sind weniger abwesend, engagieren sich mehr in der Arbeit (Freund, 2005), leisten mehr und tragen sich weniger mit dem Gedanken, die Arbeit (K. Lee, Carswell, \& Allen, 2000) oder den Beruf (Hackett \& Lapierre, 2001) wechseln zu wollen.

\subsection{Planung einer weiteren Aus- oder Weiterbildung}

In dieser Studie ist die Planung einer formalen oder branchenspezifischen, umfangreichen Aus- oder Weiterbildung von Interesse. Der Fokus liegt auf der Frage, ob durch Bildungsentscheidungen Korrekturen im Bildungsverlauf vorgenommen werden, im Sinne der Selektions- oder Gravitationsthese (Heinz, 2000).

\subsection{Hypothesen}

Personen, die einen Beruf gewählt haben oder ausüben, der besser passt, sind insgesamt zufriedener und auch stärker mit ihrem Beruf verbunden.

Hypothese 1 Je höher die Passung einer Person mit ihrem Beruf ist, desto höher ist die Zufriedenheit mit dem Beruf. Dies trifft für a) die wahrgenommene Passung und für b) die Kongruenz zu.

Hypothese 2 Je höher die Passung einer Person mit ihrem Beruf ist, desto höher ist die Verbundenheit. Dies trifft für a) die wahrgenommene Passung und auch für b) die Kongruenz zu.

Eine weitere Aus- oder Weiterbildung schafft die Voraussetzung, eine nicht passende Situation zu verbessern (Neuenschwander \& Düggeli, 2013). Entsprechend sind die folgenden Hypothesen formuliert.

Hypothese 3 Eine tiefe a) wahrgenommene Passung oder b) Kongruenz führt dazu, dass die Personen eher eine weitere Aus- oder Weiterbildung planen. 
Hypothese 4 Personen planen eine weitere Ausbildung so, dass diese besser als ihr aktueller Beruf zu ihren eigenen Interessen passt. Dies trifft für a) die wahrgenommene Passung und b) die Kongruenz zu.

\section{$2 \quad$ Methode}

Die Daten für diese Analysen stammen aus der Studie „Berufliche Entscheidungen und Entwicklungsverläufe im Jugendalter und jungen Erwachsenenalter“ (BEN). BEN ist eine auf die Zeitspanne von 2012 bis 2017 angelegte Längsschnittstudie. Die Teilnehmenden wurden mittels Online-Fragebogen befragt (Neuenschwander \& Düggeli, 2013).

Für die vorliegenden Analysen wurden Personen ausgewählt, die 2012 kurz vor Abschluss der beruflichen Grundbildung waren (Kohorte 2) und Personen, die 2012 bereits seit einigen Jahren im Erwerbsleben standen (Kohorte 3). Eine vollständige Beschreibung der Studie und aller befragten Kohorten findet sich im zweiten Zwischenbericht zum Projekt BEN (Neuenschwander, Düggeli, Nägele, \& Frey, 2015).

In Welle I liegen Antworten von 775 Personen aus Kohorte 2 und von 342 Personen aus Kohorte 3 vor. In Welle II wurden die Teilnehmenden aus Welle I wieder zur Befragung eingeladen. Zusätzlich wurden für beide Kohorten zusätzliche Teilnehmende rekrutiert, um die Stichprobe zu vergrössern. In Welle II liegen von 1'318 Personen aus Kohorte 2 und von 1'413 Personen aus Kohorte 3 Antworten vor.

Die Längsschnittstichprobe besteht aus 485 Personen. In Kohorte 2 stehen Daten von 355 Personen und in Kohorte 3 von 130 Personen zur Verfügung.

Die Personen in der Kohorte 2 waren im Jahr 2014 im Durchschnitt 22.4 Jahre alt $(S D=3.4$ Jahre), in der Kohorte 3 im Durchschnitt 28.1 Jahre alt $(S D=2.2$ Jahre).

\subsection{Messinstrumente}

Die wahrgenommene Passung wurde mit drei Items gemessen (Neuenschwander \& Frank, 2009), z. B. „Meine Arbeit passt zu meiner Person“, auf einer Antwortskale von 1 „stimmt überhaupt nicht“ bis 6 "stimmt voll und ganz". In Welle I wurde für Kohorte 2 anstelle von „Meine Arbeit..." nach „Meine Lehre..." gefragt. Die interne Konsistenz der Skala war in Welle 1 für die Passung mit der Lehre Cronbach's Alpha $=.90$, für die Passung mit dem Beruf Cronbach's Alpha $=.88$, in Welle II Cronbach's Alpha $=.88, N=1$ '781. In Welle II wurde zusätzlich die wahrgenommene Passung 
zur Aus- oder Weiterbildung erfasst. Die interne Konsistenz der Skala war in Welle II Cronbach's Alpha $=.81$.

Die Kongruenz zwischen Person und Beruf wurde mittels des C-Index nach Brown und Gore (1994) berechnet, der auf einem gewichteten Vergleich der beiden Primär-, Sekundär- und Tertiärtypen im Verhältnis zu deren Lage auf dem Hexagon beruht (Jörin Fux, 2005). Die Personentypen wurden mittels des Allgemeinen Interesse-Struktur-Tests (AIST-R) erhoben (Bergmann, 2004). Die Umwelttypen (Berufsprofile) wurden anhand der Expertenratings in Explorix (Jörin Fux et al., 2012) kodiert.

Als Outcomes werden in der vorliegenden Studie die berufliche Zufriedenheit und die Verbundenheit mit dem Beruf untersucht.

In Welle I wurde nach der Zufriedenheit mit der Lehre und der Berufsfachschule (Kohorte 2), resp. dem Betrieb und dem Beruf (Kohorte 3) gefragt, in Anlehnung an Neuenschwander et al. (2013a). Die berufliche Zufriedenheit ist der Durchschnittswert der je bereichspezifischen Zufriedenheiten. In Welle II wurde die Zufriedenheit für Kohorte 2 und Kohorte 3 für den Beruf und den Betrieb erfasst und zu einem Mass der beruflichen Zufriedenheit zusammengefasst. Die berufliche Zufriedenheit wurde in Welle I auf einer 6-stufigen Antwortskale von 1 „sehr unzufrieden“" bis 6 ,sehr zufrieden“ "erfasst. In Welle II wurde eine 7-stufige Antwortskale verwendet. Diese 7-stufige Antwortskala wurde für die Analysen in eine 6-stufige Antwortskala transformiert.

Die Verbundenheit mit dem Beruf (Commitment) wurde in Welle I mit drei Items gemessen, z.B. „Diese Ausbildung, resp. Arbeit zu haben ist wichtig“, auf einer Antwortskale von 1 „stimmt überhaupt nicht“ bis 6 „stimmt voll und ganz" (Neuenschwander, Gerber, Frank, Singer, \& Bosshard, 2013a). Die interne Konsistenz der Skala war Cronbach's Alpha $=.79$. In Welle II wurde die Verbundenheit mit dem Beruf (Commitment) in Anlehnung an Allen und Meyer (1990), in einer Adaptation von Nübling (2005) mit drei Items gemessen, z. B. „Stolz, diesem Betrieb anzugehören“, auf einer Antwortskale von 1 ,stimmt überhaupt nicht“ bis 6 ,stimmt voll und ganz". Die interne Konsistenz der Skala war Cronbach's Alpha $=.85$.

Die Planung einer Aus- oder Weiterbildung wurde in Welle I für die Kohorte 2 mit zwei Fragen erfasst (Antwortoptionen ja-nein): „Erwägen Sie eine Aus- oder Weiterbildung?" und „Haben Sie diese Aus- oder Weiterbildung verbindlich geplant?" (Neuenschwander, Hermann, Frank, \& Faschinger, 2013b). Die Planung einer weiteren Ausbildung wurde für Kohorte 3 in einer Frage mit den Antwortoptionen erfasst: „Im Moment habe ich keine konkreten Pläne, eine Aus- oder Weiterbildung zu beginnen“, „Ich plane eine Aus- oder Weiterbildung zu beginnen“ und „Ich werde sicher eine Aus- oder Weiterbildung beginnen "(Neuenschwander, Hermann, Frank, \& Faschinger, 2013b). Diese Variable wurde in zwei Kategorien zusammengefasst, 
1 „keine Planung“ und 2 „Planung oder beginnen“. In Welle II wurde die geplante weitere Ausbildung für beide Kohorten gleich erfragt wie bei Kohorte 3 in Welle I.

Bildungsentscheidungen werden durch das Geschlecht (Berweger, Kappler, Keck Frei, \& Bieri Buschor, 2015) und strukturelle Merkmale beeinflusst. Das über die Kohorte erfasste strukturelle Merkmal ist der aktuelle Stand der Laufbahn. Dies ist für Kohorte 2 der Übergang von der beruflichen Grundbildung in die berufliche Tätigkeit und für Kohorte 3 die allfällige Planung einer weiteren Aus- oder Weiterbildung.

\section{$3 \quad$ Resultate}

\subsection{Deskriptive Ergebnisse}

In Tabelle 1 sind die Mittelwerte, Standardabweichungen und Korrelationen für die Welle I, die Welle II und den Längsschnitt dokumentiert.

Die Mittelwerte der Personen, die sich an beiden Wellen beteiligten, unterscheiden sich von denen, die sich nur an Welle I, respektive nur an Welle II beteiligten, teilweise systematisch. Die Effektstärken sind jedoch gering (Cohen's $d<.30$ ). Die Personen der Längsschnittstichprobe waren mehr mit ihrem Beruf verbunden, hatten eine etwas höhere Kongruenz mit der Aus- oder Weiterbildung und waren auch etwas zufriedener mit der Arbeit als die übrigen Teilnehmenden in Welle I oder Welle II.

Tab. 1 Deskriptive Ergebnisse und Korrelationen

\begin{tabular}{|c|c|c|c|c|c|c|c|c|c|c|c|}
\hline Welle I/Welle II & MW & SD & 1 & 2 & 3 & 4 & 5 & 6 & 7 & 8 & 9 \\
\hline 1 Geschlecht & $1.49 / 1.42$ & $.50 / .49$ & 1 & -.01 & -.06 & $-.26^{* *}$ & $-.08^{*}$ & . & $-.12^{* *}$ & & . \\
\hline 2 Kohorte & $2.31 / 2.52$ & $.46 / .50$ & .02 & 1 & $.09^{* *}$ & -.20 & $.60^{* *}$ & . & $-.10^{* *}$ & & \\
\hline 3 Zufriedenheit & $4.76 / 5.10$ & $.75 / 1.18$ & .04 & -.02 & 1 & $.19^{* *}$ & $.09^{* *}$ & . & $.60^{* *}$ & & . \\
\hline 4 Verbundenheit & $5.04 / 4.37$ & $.89 / 1.04$ & .05 & -.01 & $.44^{* *}$ & 1 & $.73^{*}$ & . & $.22^{\star *}$ & & . \\
\hline 5 Planung & $1.41 / 1.46$ & $.49 / .50$ & $.05^{*}$ & $-.21^{* *}$ & -.04 & -.02 & 1 & . & -.03 & & . \\
\hline 6 Kongruenz &.$/ 10.91$ & .13 .66 & .02 & .03 & .04 & $.09^{* *}$ & -.02 & 1 & & & . \\
\hline $\begin{array}{l}7 \text { Wahrgenom- } \\
\text { mene Passung }\end{array}$ & $4.85 / 4.62$ & $.93 / 1.01$ & .02 & .03 & $.46^{* *}$ & $.67^{* *}$ & $-.08^{* *}$ & $.11^{* *}$ & 1 & & . \\
\hline 8 Differenz W & .1 .52 &.$/ 1.12$ & -.07 & .03 & $-.35^{* *}$ & $-.56^{* *}$ & .08 & $-.13^{*}$ & $-.78^{* *}$ & 1 & . \\
\hline 9 Differenz K & .1 .53 &.$/ 4.41$ & -.01 & -.07 & -.05 & -.04 & -.08 & $-.59^{* *}$ & -.08 & .01 & 1 \\
\hline
\end{tabular}

Bemerkung. Welle I oberhalb der Diagonale, N zwischen 985 und 1055; Welle II (kursiv) unterhalb der Diagonale $N$ zwischen 368 und 2635. ${ }^{\star} p<.05,{ }^{* *} p<.01$, ,." $=$ keine Daten verfügbar 


\begin{tabular}{lccccccccc}
\hline Längsschnitt & \multicolumn{1}{c}{ Welle II } \\
\hline Welle I & MW & \multicolumn{1}{c}{ SD } & 1 & 2 & 3 & \multicolumn{1}{c}{4} & \multicolumn{1}{c}{5} & \multicolumn{1}{c}{6} & \multicolumn{1}{c}{7} \\
\hline 1 Geschlecht & 1.45 & .50 & 1 & .09 & .03 & $.13^{*}$ & -.02 & .01 & $-.13^{* *}$ \\
2 Kohorte & 2.27 & .44 & .09 & 1 & -.03 & .05 & -.01 & .01 & .08 \\
3 Zufriedenheit & $4.76 / 5.28$ & $.70 / 1.06$ & -.02 & -.05 & $.21^{* *}$ & $.20^{* *}$ & -.05 & .01 & $.27^{* *}$ \\
4Verbundenheit & $5.11 / 4.27$ & $.81 / 1.02$ & $-.25^{* *}$ & -.04 & .04 & -.10 & .03 & -.13 & -.05 \\
5 Planung & $1.44 / 1.48$ & $.50 / .50$ & -.02 & $-.63^{* *}$ & -.02 & .05 & -.04 & .02 & .08 \\
6 Kongruenz &.$/ 11.42$ &.$/ 3.50$ &. &. &. &. &. &. &. \\
7 Wahrgenom- & $4.90 / 4.59$ & $.91 / .92$ & $-.10^{*}$ & $-.13^{* *}$ & .10 & .08 & -.04 & .17 & .01 \\
mene Passung & & & & & & & & & \\
\hline
\end{tabular}

Bemerkung. Kursiv = Welle II, N zwischen 995 und 116; Längsschnitt N zwischen 159 und 485. ${ }^{\star} \mathrm{p}<.05,{ }^{* *} \mathrm{p}<.01, .=$ keine Daten verfügbar

\subsection{Planung einer Aus- oder Weiterbildung}

In Tabelle 2 ist aufgeführt, wie viele Personen eine Aus- oder Weiterbildung in Welle I und Welle II planten. Auffällig ist, dass in Welle I in der Kohorte 2 deutlich mehr Personen keine Aus- oder Weiterbildung planten als erwartet $(N=611$, erwartet 459, adj. Residual -20.0). In Welle II planten hingegen mehr Personen der Kohorte 2 als erwartet eine Aus- oder Weiterbildung $(N=665$, erwartet 535, adj. Residual 5.6).

Tab. 2 Planung einer weiteren Aus- oder Weiterbildung

\begin{tabular}{llrrrr}
\hline & Planung & \multicolumn{2}{c}{ Welle I } & \multicolumn{2}{c}{ Welle II } \\
\hline Kohorte 2 & Nein & 611 & $79 \%$ & 512 & $44 \%$ \\
& Ja & 164 & $21 \%$ & 665 & $56 \%$ \\
Total & & 775 & $100 \%$ & $1^{\prime} 117$ & $100 \%$ \\
\hline Kohorte 3 & Nein & 51 & $15 \%$ & 817 & $65 \%$ \\
& Ja & 291 & $85 \%$ & 446 & $35 \%$ \\
\hline Total & & 342 & $100 \%$ & 1 '263 & $100 \%$ \\
\hline
\end{tabular}

Bemerkungen. Welle I: Chi-Quadrat $(1)=401.67, p<.01$. Welle II: Chi-Quadrat $(1)=110.27, p<.01$

In Welle I planten insgesamt mehr Frauen als erwartet eine weitere Aus- oder Weiterbildung $(N=236$, erwartet 217 , adj. Residual 2.4), was sich aber in Welle II umkehrte. Es gab in Welle II mehr Männer als erwartet, die eine weitere Aus- oder Weiterbildung planten ( $N=462$, erwartet 435, adj. Residual 2.3) (Tabelle 3). 
Tab. 3 Planung nach Geschlecht

\begin{tabular}{lllrrr}
\hline & Planung & Welle I & \multicolumn{3}{c}{ Welle II } \\
\hline \multirow{2}{*}{ Frauen } & nein & 294 & $56 \%$ & 792 & $56 \%$ \\
& ja & 236 & $44 \%$ & 613 & $44 \%$ \\
\hline \multirow{2}{*}{ Total } & & 530 & $100 \%$ & 1 '405 & $100 \%$ \\
\hline \multirow{2}{*}{ Männer } & nein & 322 & $63 \%$ & 493 & $52 \%$ \\
& ja & 190 & $37 \%$ & 462 & $48 \%$ \\
\hline Total & & 512 & $100 \%$ & 955 & $100 \%$ \\
\hline
\end{tabular}

Bemerkungen. Welle I: Chi-Quadrat $(1)=5.93, p<.02$. Welle II: Chi-Quadrat $(1)=110.27, p<.01$

\subsection{Prüfung der Hypothesen}

In Hypothese 1 ist formuliert, dass eine höhere Passung einer Person mit ihrem Beruf positiv mit der beruflichen Zufriedenheit zusammenhängt. Die Ergebnisse in Tabelle 4 zeigen, dass die berufliche Zufriedenheit in Welle I mit der wahrgenommenen Passung und der Kohorte zusammenhing. In Welle II zeigte sich ein Effekt der wahrgenommenen Passung, und im Längsschnitt war es die wahrgenommene Passung in

Tab. 4 Prädiktoren der beruflichen Zufriedenheit

\begin{tabular}{|c|c|c|c|c|c|c|c|c|c|c|c|c|c|c|c|}
\hline & \multicolumn{5}{|c|}{ Welle I } & \multicolumn{5}{|c|}{ Welle II } & \multicolumn{5}{|c|}{ Längsschnitt } \\
\hline & B & S.F. & Beta & $\mathrm{t}$ & sig. & B & S.F. & Beta & $\mathrm{t}$ & sig. & B & S.F. & Beta & $\mathrm{t}$ & sig. \\
\hline Geschlecht & .03 & .04 & .02 & .80 & .42 & .07 & .06 & .03 & 1.05 & .29 & .12 & .19 & .05 & .64 & .52 \\
\hline Kohorte & .25 & .04 & .15 & 5.93 & $<.01$ & -.01 & .06 & -.01 & -.22 & .83 & -.04 & .20 & -.02 & -.21 & .84 \\
\hline $\begin{array}{l}\text { Berufliche } \\
\text { Zufrieden- } \\
\text { heit WI }\end{array}$ & & & & & & & & & & & .36 & .18 & .22 & 1.98 & .05 \\
\hline $\begin{array}{l}\text { Wahrge- } \\
\text { nommene } \\
\text { Passung } \\
\text { WI }\end{array}$ & .50 & .02 & .62 & 24.61 & $<.01$ & & & & & & -.18 & .15 & -.14 & -1.23 & .22 \\
\hline $\begin{array}{l}\text { Kongruenz } \\
\text { WII }\end{array}$ & & & & & & -.01 & .01 & -.02 & -.63 & .53 & -.03 & .03 & -.10 & -1.29 & .20 \\
\hline $\begin{array}{l}\text { Wahrge- } \\
\text { nommene } \\
\text { Passung } \\
\text { WII }\end{array}$ & & & & & & .53 & .03 & .45 & 17.31 & $<.01$ & .41 & .11 & .33 & 3.87 & $<.01$ \\
\hline
\end{tabular}

Bemerkungen.; Welle I $N=983: F(3,979)=207.22, p<.01, \mathrm{R}^{2}=.39$. Für Welle I liegt kein Mass für die Kongruenz vor, die Interessen wurden ab Welle II erfasst. Welle II $N=1^{\prime} 213$ : $F(4,1208)=75.94, p<.01, \mathrm{R}^{2}=.20$. Längsschnitt $N=144: F(6,137)=4.72,<=.01, \mathrm{R}^{2}=.14$. S.F.: Standardfehler 
Welle II, welche zur Erklärung der Varianz in der beruflichen Zufriedenheit beitrug. Die Teilnehmenden der Kohorte 2 waren zum Zeitpunkt der Befragung kurz vor Abschluss ihrer Lehre. Dies kann erklären, weshalb die Kohorte einen Einfluss auf die berufliche Zufriedenheit hatte. Im Längsschnitt war ein wichtiger Prädiktor der beruflichen Zufriedenheit in Welle II die berufliche Zufriedenheit in Welle I.

Die Hypothese 1a kann nur teilweise bestätigt werden. Querschnittlich erklärt die wahrgenommene Passung die Zufriedenheit mit dem Beruf, längsschnittlich jedoch nicht. Die Kongruenz Person-Beruf hat keinen Einfluss auf die Zufriedenheit, Hypothese $1 \mathrm{~b}$ ist nicht bestätigt.

In Hypothese 2 ist formuliert, dass eine höhere Passung einer Person mit ihrem Beruf mit einer höheren Bindung an den Beruf (Commitment) zusammenhängt. Die Ergebnisse in Tabelle 5 zeigen, dass die Verbundenheit mit dem Beruf in Welle I mit der wahrgenommenen Passung und dem Geschlecht zusammenhing. Das negative Vorzeichen beim Beta des Geschlechts bedeutet, dass die Männer in Welle I eher mit ihrem Beruf verbunden waren als die Frauen. In Welle II hing die Verbundenheit mit dem Beruf mit der wahrgenommenen Passung zusammen, wie auch im Längsschnitt. Im Längsschnitt ist auffallend, dass keine Prädiktoren aus der Welle I zur Vorhersage der Verbundenheit mit dem Beruf signifikant wurde.

Tab. 5 Prädiktoren der Verbundenheit mit dem Beruf

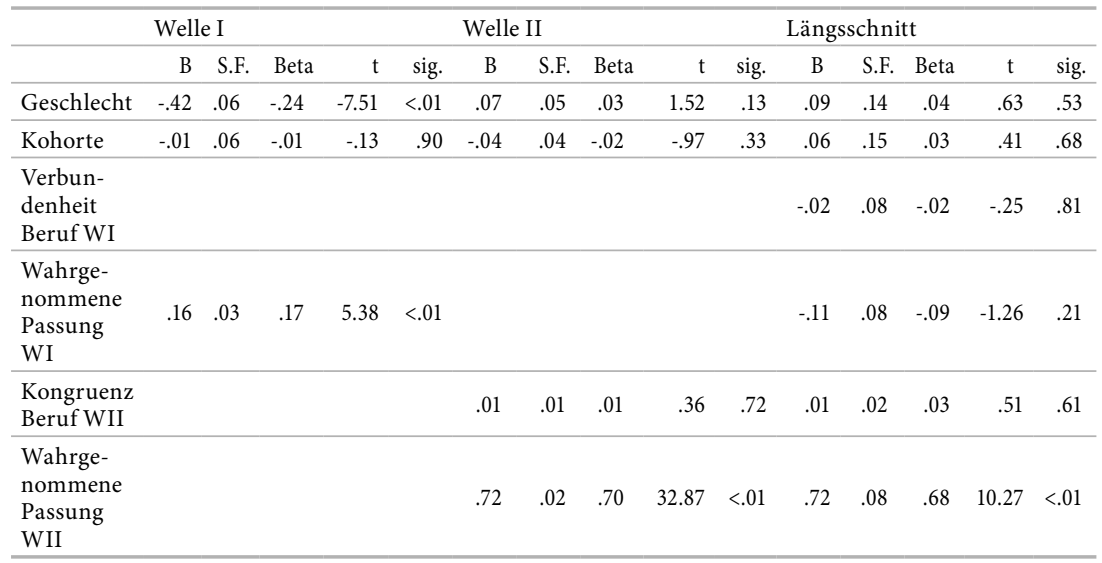

Bemerkung. Welle I $N=924: F(2,921)=33.46, p<.01, \mathrm{R}^{2}=.07$; Modell 2: $F(3,920)=32.62$, $p<.01, \mathrm{R}^{2}=.09$, Welle II $N=1$ '214: $F(4,1209)=275.51, p<.01, \mathrm{R}^{2}=.48$. Längsschnitt $N=142: F(6,135)=20.07,<=.01, \mathrm{R}^{2}=.45$. S.F.: Standardfehler 
Die Hypothese 2a kann nur teilweise bestätigt werden. Querschnittlich erklärt die wahrgenommene Passung die Verbundenheit mit dem Beruf, längsschnittlich jedoch nicht. Die Kongruenz Person-Beruf hat keinen Einfluss auf die Verbundenheit, Hypothese $2 \mathrm{~b}$ ist nicht bestätigt.

In Hypothese 3 ist formuliert, dass eine tiefe wahrgenommene Passung oder Kongruenz dazu führen, dass die Personen eher eine Aus- oder Weiterbildung planen. Die Ergebnisse in Tabelle 6 zeigen, dass in Welle I die Planung einer weiteren Aus- und Weiterbildung vor allem durch das Geschlecht bestimmt war. In Welle I planten Frauen mit einer höheren Wahrscheinlichkeit eine Aus- oder Weiterbildung als die Männer. In Welle II dreht sich dieser Geschlechtseffekt um. Nun waren es die Männer, die eher eine Aus- oder Weiterbildung planten. Zudem führte eine höhere wahrgenommene Passung dazu, eher keine Aus- oder Weiterbildung zu planen. Längsschnittlich kann kein Prädiktor einen Beitrag zur Erklärung der Planung leisten.

Tab. 6 Prädiktoren der Planung einer weiteren Aus- oder Weiterbildung

\begin{tabular}{|c|c|c|c|c|c|c|c|c|c|c|c|c|}
\hline & \multicolumn{4}{|c|}{ Welle I } & \multicolumn{4}{|c|}{ Welle II } & \multicolumn{4}{|c|}{ Längsschnitt } \\
\hline & $\operatorname{Exp}(B)$ & $\begin{array}{l}\text { CFI } \\
95 \%\end{array}$ & & sig. & $\operatorname{Exp}(B)$ & $\begin{array}{r}\text { CFI } \\
95 \%\end{array}$ & & sig. & $\operatorname{Exp}(B)$ & $\begin{array}{l}\text { CFI } \\
95 \%\end{array}$ & & sig. \\
\hline Geschlecht & 1.78 & 1.25 & 2.53 & $<.01$ & .77 & .60 & .98 & .03 & .52 & .25 & 1.06 & .07 \\
\hline Kohorte & .01 & .00 & .02 & $<.01$ & 2.44 & 1.92 & 3.08 & $<.01$ & 1.28 & .42 & 3.92 & .66 \\
\hline Planung WI & & & & & & & & & .76 & .27 & 2.13 & .60 \\
\hline Passung WI & 1.12 & .91 & 1.38 & .27 & & & & & .63 & .41 & .96 & .03 \\
\hline $\begin{array}{l}\text { Kongruenz } \\
\text { Beruf WII }\end{array}$ & & & & & .99 & .96 & 1.03 & .72 & 1.05 & .95 & 1.15 & .37 \\
\hline $\begin{array}{l}\text { Passung } \\
\text { WII }\end{array}$ & & & & & .88 & .78 & 1.03 & .04 & .98 & .98 & 1.43 & .89 \\
\hline
\end{tabular}

Bemerkung. Welle I $N=985:-2$ Log likelihood $=826.23$, Chi-Quadrat $(3)=517.85, p<.01$, Nagelkerke $\mathrm{R}^{2}=.55$. Welle II $N=1213:-2$ Log likelihood = 1605.93, Chi-Quadrat $(4)=64.73$, $p<.01$, Nagelkerke $\mathrm{R}^{2}=.07$. Längsschnitt II $N=1213:-2$ Log likelihood $=191.10$, Chi-Quadrat $(6)=8.27, p=.22$, Nagelkerke $\mathrm{R}^{2}=.07$

Hypothese 3a wurde teilweise bestätigt, da eine tiefere wahrgenommene Passung in Welle II die Wahrscheinlichkeit erhöhte, eine Aus- oder Weiterbildung zu planen. Hypothese $3 \mathrm{~b}$ kann nicht bestätigt werden, da die Kongruenz Person-Beruf keinen Einfluss auf die Planung einer Aus- oder Weiterbildung hatte.

In Hypothese 4 wird formuliert, dass Personen eine Aus- oder Weiterbildung so wählen, dass diese passender als der aktuelle Beruf ist. Dazu wurde ein Differenzwert der Passung zwischen der Aus- und Weiterbildung und der Passung mit 
dem Beruf berechnet. Je grösser diese Differenz ist, desto unähnlicher sind sich der Beruf und die Aus- oder Weiterbildung. Der Differenzwert wurde zuerst für die wahrgenommene Passung der Aus- oder Weiterbildung und der wahrgenommenen Passung zum Beruf bezüglich der geplanten Aus- und Weiterbildung berechnet. Der Differenzwert lag bei $M W=.52, S D=1.12, N=483$. Zweitens wurde zur Berechnung des Differenzwerts für die Kongruenz Person-Beruf die Kongruenz Person-besuchte Aus- oder Weiterbildung berechnet. Dieser Differenzwert liegt bei $M W=.53, S D=4.41, N=383$. Im Durchschnitt planten die befragten Personen also eher eine Aus- oder Weiterbildung mit einer höheren Kongruenz.

In Tabelle 7 sind zuerst die Ergebnisse zur Erklärung der Differenzwerte der wahrgenommenen Passung aufgeführt. Die Differenzwerte können durch das Geschlecht, die Kohorte und die Kongruenz Person-Beruf nicht vorhergesagt werden. Signifikant wird die wahrgenommene Passung. Personen mit einer tiefen Passung planen eine Aus- oder Weiterbildung, die besser zu ihnen passt. Dies gilt jedoch nur bis zu einem Grenzwert. Aufgrund der Regressionsgleichung planten Personen mit einer wahrgenommenen Passung von mehr als 5.21 eine Aus- oder Weiterbildung, die weniger gut passte.

In Tabelle 7 sind zweitens die Ergebnisse zur Erklärung der Differenz der Kongruenz der besuchten Aus- oder Weiterbildung und des aktuellen Berufs aufgeführt. Diese Differenz wird durch die Kongruenz Person-Beruf erklärt. Sie wird kleiner, je grösser die Kongruenz Person-Beruf ist. Personen mit einer tiefen Kongruenz planten also eine Aus- oder Weiterbildung, die deutlich kongruenter war als ihr Beruf. Aufgrund der Regressionsgleichung kann gezeigt werden, dass Personen mit einer Kongruenz Person-Beruf von weniger als 11.5 eine weitere Aus- oder Weiterbildung planten, die besser passte, bei einer Kongruenz von mehr als 11.5 aber eine weitere Aus- oder Weiterbildung, die weniger gut passte.

Tab. 7 Prädiktoren des Differenzwerts

\begin{tabular}{lcccccccccc}
\hline Welle II & \multicolumn{1}{c}{ Differenzwert Wahrgenommene Passung } & \multicolumn{5}{c}{ Differenzwert Kongruenz } \\
\hline & B & S.F. & Beta & $\mathrm{t}$ & sig. & B & S.F. & Beta & $\mathrm{t}$ & sig. \\
\hline Geschlecht & -.04 & .08 & -.02 & -.46 & .64 & .06 & .40 & .01 & .15 & .47 \\
\hline Kohorte & .10 & .01 & .05 & 1.27 & .20 & -.29 & .39 & -.03 & -.89 & .39 \\
\hline $\begin{array}{l}\text { Kongruenz } \\
\text { Beruf WII }\end{array}$ & .01 & .01 & .05 & 1.28 & .20 & -.68 & -.05 & -.55 & -12.59 & $<.01$ \\
\hline $\begin{array}{l}\text { Passung } \\
\text { Beruf WII }\end{array}$ & -.83 & .04 & -.78 & -21.44 & $<.01$ & -.17 & .19 & -.04 & -.87 & .39 \\
\hline Konstante & 3.96 & .29 & & 13.09 & $<.01$ & 9.20 & 1.48 & & 6.22 & $<.01$ \\
\hline
\end{tabular}

Bemerkung. Wahrgenommene Passung: $N=336: F(4,331)=118.29, p<.01, \mathrm{R}^{2}=.58$. Kongruenz $N=336: F(4,361)=41.22, p<.01, \mathrm{R}^{2}=.31$ 
Die Hypothesen 4a und 4b können teilweise bestätigt werden, da Personen eine weitere Aus- oder Weiterbildung wählten, die besser passte oder kongruenter war. Dies gilt jedoch nur bis zu einem bestimmten Grenzwert. Wird dieser überschritten, wählten die Personen weniger passende oder weniger kongruente Aus- oder Weiterbildungen.

\section{$4 \quad$ Diskussion}

Die auf dem Person-Umwelt-Modell (Holland, 1997; Super, 1980) basierende Annahme, dass eine hohe Kongruenz zu einer höheren Zufriedenheit und einer stärkeren Verbundenheit führt, konnte nicht bestätigt werden. Hingegen konnte ein deutlicher Zusammenhang mit der subjektiven, wahrgenommenen Passung (Kristof-Brown et al., 2005) gezeigt werden. Im Längsschnitt zeigte sich, dass die Zufriedenheit mit dem Beruf in Welle II von der Zufriedenheit in der Welle I abhing, was auf eine Stabilität der Zufriedenheit mit dem Beruf über die Zeit hinweist (Keller \& Semmer, 2013). Die berufliche Zufriedenheit und Verbundenheit scheinen sich also nicht aufgrund dispositionaler Faktoren (Interessen), sondern aufgrund einer subjektiven Einschätzung der Qualität des Anpassungsprozesses zu entwickeln (Nägele \& Neuenschwander, 2016). Der fehlende Einfluss der Verbundenheit mit dem Beruf in Welle I auf die Verbundenheit mit dem Beruf in Welle II ist durch die Veränderung des Messinstruments gut zu erklären.

Weder die wahrgenommene Passung noch die Kongruenz sind gute Prädiktoren der Planung einer weiteren Aus- oder Weiterbildung. Die Planung einer Aus- und Weiterbildung unterscheidet sich in Welle I und Welle II. In Welle I scheinen vor allem die Männer der Kohorte 2 die Planung der Aus- oder Weiterbildung aufzuschieben. Dies kann damit erklärt werden, dass einige junge Männer nach Abschluss der beruflichen Grundbildung zuerst den Militärdienst absolvieren und die Planung einer weiteren Aus- oder Weiterbildung aufschieben. Frauen finden sich eher in Gesundheitsberufen, in denen eine frühe und kontinuierliche formale Ausbildung einen höheren Stellenwert hat als in vielen handwerklichen Berufen (Trede, 2016). Wenn ein Effekt auf die Planung einer Aus- oder Weiterbildung auftritt, dann ist es ein Effekt der wahrgenommenen Passung, nicht aber der Kongruenz Person-Beruf. Je höher die Passung, desto weniger wahrscheinlich ist die Planung. In Welle II ist dieser Effekt der aktuellen Passung auf die Planung einer Aus- oder Weiterbildung deutlich zu sehen. Im Längsschnitt lässt sich die Planung nicht vorhersagen. Das heisst, dass vor allem eine aktuell als schlechte wahrgenommen Passung Planungsaktivitäten auslöst. 
Personen mit einer insgesamt tiefen Passung zum Beruf oder mit einer geringen Kongruenz Person-Beruf wählen eine Aus- oder Weiterbildung so, dass diese besser passt, resp. kongruenter ist. Personen mit einer bereits hohen wahrgenommenen Passung oder hohen Kongruenz wählen eine Aus- oder Weiterbildung, die eher weniger zu ihnen passte. Dies heisst, dass nur Personen mit einem unter einem bestimmten Grenzwert liegenden Niveau der Passung versuchen, eine geringe Passung Person-Beruf zu verbessern. Dieses Ergebnis kann aufgrund der vorliegenden Daten unterschiedlich interpretiert werden. So ist es möglich, dass Personen mit einer bereits sehr hohen Passung zum Beruf kaum eine Aus- oder Weiterbildung finden können, die noch besser passt. Es ist auch möglich, dass diese Personen aufgrund biographischer Ereignisse gezwungen sind, ihren Beruf zu wechseln. Dies zum Beispiel aus gesundheitlichen oder familiären Gründen. Weitere Analysen mit Daten zum Bildungsverlauf, die aktuell noch nicht vorliegen, werden hier Antworten ermöglichen.

Die querschnittlichen und längsschnittlichen Befunde unterscheiden sich teilweise. Da die beiden Erhebungszeitpunkte zwei Jahre auseinanderliegen, kann dies als Hinweis interpretiert werden, dass die Planung einer Aus- oder Weiterbildung bei den Jugendlichen und jungen Erwachsenen stark auch von aktuellen Ereignissen beeinflusst wird und nicht von Ereignissen und Einschätzungen von vor zwei Jahren.

Einschränkungen. Es liegen nicht zu allen Variablen Daten im Längsschnitt vor, so dass einige Ergebnisse auf querschnittlichen Analysen beruhen. Die befragten Personen bilden eine relativ homogene Gruppe mit einer im Durchschnitt hohen Passung und Kongruenz zu ihrem Beruf. Die Längsschnittstichprobe ist im Vergleich zur Stichprobe in Welle I und Welle II relativ klein, was geschlechts-, und kohortenspezifische Auswertungen erschwert. Deshalb werden in diesem Kapitel keine Hypothesen zu Geschlecht und Kohorte formuliert.

Zusammenfassung. Es scheint wichtig, der subjektiven Einschätzung der Passung zum Beruf einen hohen Stellenwert zu geben. Personen mit einer tiefen wahrgenommenen Passung sind weniger zufrieden und weniger an den Beruf gebunden, und sie planen eher eine weitere Aus- und Weiterbildung. Wenn Personen eine Aus- oder Weiterbildung planen, dann planen sie diese so, dass sie besser zu ihnen passt, sofern die Passung Person-Beruf tief ist. Die besuchte Weiterbildung passt besser zu den eigenen Interessen, wenn sich diese Interessen und der bisherige Beruf schlecht entsprechen. Bei einer hohen wahrgenommenen Passung oder Kongruenz Person-Beruf findet sich dieser Effekt nicht mehr. Die Planung einer Aus- und Weiterbildung ist von der aktuellen Dynamik im Beruf abhängig. Wenn eine Aus- oder Weiterbildung geplant wird, so erfolgt dies aufgrund zeitlich naher Ereignisse und Einschätzungen. Das heisst aber auch, dass so lange die Situation als subjektiv passend wahrgenommen wird, die Planung einer Aus- oder Weiterbildung 
weniger wahrscheinlich ist. Erst wenn die Situation als unpassend wahrgenommen wird, kommt es zu einer Planung. Bei Personen, die eine Aus- oder Weiterbildung umsetzen, wird diese so gewählt, dass sie kongruenter ist, das heisst, besser zu den Interessen passt. Das heisst, dass die Planung einer Aus- oder Weiterbildung wahrscheinlich durch eine subjektiv wahrgenommene Verschlechterung der Passung ausgelöst wird. Welche Aus- oder Weiterbildung gemacht wird, wird dann aber durch die Interessen mit beeinflusst.

Die Passung wird als ein wichtiger Faktor diskutiert, der nicht nur die Berufsfindung, sondern auch die Entwicklung im Beruf erklären soll. Die in diesem Kapitel präsentierten Ergebnisse zeigen, dass die wahrgenommene Passung, also die Selbsteinschätzung, ob man sich im passenden Beruf oder der passenden Ausbildung befindet, einen sehr grossen Einfluss auf die Zufriedenheit und die Verbundenheit mit dem Beruf hat. Die Kongruenz spielt dabei keine Rolle.

\section{Literatur}

Allen, N. J., \& Meyer, J. P. (1990). The measurement and antecedents of affective, continuance and normative commitment to the organization. Journal of Occupational Psychology, 63, 1-18.

Bauer, T. N., \& Erdogan, B. (2011). Organizational socialization: The effective onboarding of new employees. In S. Zedeck (Ed.), APA handbook of industrial and organizational psychology (Vol. III, pp. 51-64). Washington, DC: American Psychological Association. http://doi.org/10.1037/12171-002

Bauer, T. N., Bodner, T., Erdogan, B., Truxillo, D. M., \& Tucker, J. S. (2007). Newcomer adjustment during organizational socialization: A meta-analytic review of antecedents, outcomes, and methods. Journal of Applied Psychology, 92(3), 707-721. http://doi. org/10.1037/0021-9010.92.3.707

Bergmann, C. (2004). Berufswahl. In H. Schuler (Hrsg.), Organisationspsychologie - Grundlagen und Personalpsychologie (S. 343-388). Göttingen, D: Hogrefe Verlag für Psychologie.

Berweger, S., Kappler, C., Keck Frei, A., \& Bieri Buschor, C. (2015). Geschlechtsuntypische Laufbahnpläne. Schweizerische Zeitschrift für Bildungswissenschaften, 37(2), 321-339.

Brown, S. D., \& Gore, P. A., Jr. (1994). An evaluation of interest congruence indices: Distribution characteristics and measurement properties. Journal of Vocational Behavior, 45, 310-327.

Cable, D. M., \& Judge, T. A. (1996). Person-organization fit, job choice decisions, and organizational entry. Organizational Behavior and Human Decision Processes, 67, 294-311. http://doi.org/10.1037/0021-9010.92.5.1446

Cohen, A. (2007). Dynamics between occupational and organizational commitment in the context of flexible labor markets: A review of the literature and suggestions for a future research agenda. Bremen, D: Universität Bremen, Institut Technik und Bildung. 
Eccles, J. S., Midgley, C., Wigfield, A., Buchanan, C. M., Reuman, D., Flanagan, C., \& Mac Iver, D. (1993). Development during adolescence: The impact of stage-environment fit on young adolescents' experiences in schools and in families. American Psychologist, $48(2), 90-101$.

Freund, A. (2005). Commitment and job satisfaction as predictors of turnover intentions among welfare workers. Administration in Social Work, 29(2), 5-21. http://doi.org/10.1300/ J147v29n02_02

Frey, S., Neuenschwander, M. P., \& Nägele, C. (2016). Intention to participate in continuing education of young adults planning to have children concurrently Individual and situational aspects. Presented at the European Conference on Educational Resarch, Dublin, IE 23-26 August.

Grant, A. M., \& Ashford, S. J. (2008). The dynamics of proactivity at work. Research in Organizational Behavior, 28, 3-34.

Hackett, R. D., \& Lapierre, L. M. (2001). Understanding the links between work commitment constructs. Journal of Vocational Behavior, 58(3), 392-413. http://doi.org/10.1006/ jvbe. 2000.1776

Hansen, J.-I. C., \& Lee, W. V. (2007). Person-vocation fit. In S. G. Rogelberg (Ed.), Encyclopedia of Industrial and Organizational Psychology (pp. 621-623). Thousand Oaks, CA: SAGE Publications.

Heinz, W. R. (2000). Selbstsozialisation im Lebenslauf. Umrisse einer Theorie biografischen Handelns. In E. M. Hoerning (Hrsg.), Biografische Sozialisation (S. 165-186). Stuttgart, D: Lucius \& Lucius.

Holland, J. L. (1997). Making vocational choices. A theory of vocational personalities and work environments (3rd ed.). Odessa: Psychological Assessment Resources.

Jörin Fux, S. (2005). Persönlichkeit und Berufstätigkeit: Theorie und Instrumente von John Holland im deutschsprachigen Raum, unter Adaptation und Weiterentwicklung von Self-directed Search (SDS) und Position Classification Inventory (PCI). Göttingen, D: Cuvillier Verlag.

Jörin Fux, S., Stoll, F., Bergmann, C., Eder, F., \& Hell, B. (2012). EXPLORIX ${ }^{\oplus}$ - Das Werkzeug zur Berufswahl und Laufbahnplanung. Deutschsprachige Adaption und Weiterentwicklung des Self-Directed Search ${ }^{\varpi}$ (SDS) nach John L. Holland. Bern, CH: Verlag Hans Huber.

Judge, T. A. (2007). Perspectives on organizational fit. New York, NY: Psychology Press, Taylor \& Francis Group.

Kammeyer-Mueller, J. D., \& Wanberg, C. R. (2003). Unwrapping the organizational entry process: disentangling multiple antecedents and their pathways to adjustment. The Journal of Applied Psychology, 88(5), 779-794. http://doi.org/10.1037/0021-9010.88.5.779

Keller, A. C., \& Semmer, N. K. (2013). Changes in situational and dispositional factors as predictors of job satisfaction. Journal of Vocational Behavior, 83(1), 88-98. http://doi. org/10.1016/j.jvb.2013.03.004

Kristof, A. L. (1996). Person-organization fit: An integrative review of its conceptualizations, measurement, and implications. Personnel Psychology, 49(23), 1-49. http://doi. org/10.1007/978-3-531-90820-5

Kristof-Brown, A. L., Zimmerman, R. D., \& Johnson, E. C. (2005). Consequences of individuals' fit at work: A meta-analysis of person-job, person-organization, person-group, and person-supervisor fit. Personnel Psychology, 58, 281-342. 
Lee, K., Carswell, J. J., \& Allen, N. J. (2000). A meta-analytic review of occupational commitment: Relations with person- and work-related variables. Journal of Applied Psychology, 85(5), 799-811. http://doi.org/10.1037/0021-9010.85.5.799

Nägele, C., \& Neuenschwander, M. P. (2015). Passt der Beruf zu mir? Determinanten und Konsequenzen wahrgenommener Passung mit dem Lehrberuf beim Übergang in die Berufsbildung. In K. Häfeli, M. P. Neuenschwander, \& S. Schumann (Hrsg.), Berufliche Passagen im Lebenslauf (S.49-74). Wiesbaden, D: Springer Fachmedien. http://doi. org/10.1007/978-3-658-10094-0_3

Nägele, C., \& Neuenschwander, M. P. (2016). Apprentice-trainer relationship and work group integration in the first months of an apprenticeship. Empirical Research in Vocational Education and Training, 8(1), 4. http://doi.org/10.1186/s40461-016-0030-3

Neuenschwander, M. P., \& Düggeli, A. (2013). BEN Projektantrag: Berufliche Entscheidungen und Entwicklungsverläufe im Jugendalter und jungen Erwachsenenalter. Solothurn, $\mathrm{CH}$ : Fachhochschule Nordwestschweiz, Pädagogische Hochschule.

Neuenschwander, M. P., \& Frank, N. (2009). Familie-Schule-Beruf FASE B. Dokumentation der Schülerbefragung Erhebung 2008. Solothurn, CH: Fachhochschule Nordwestschweiz, Pädagogische Hochschule, Institut Forschung und Entwicklung, Zentrum Lernen und Sozialisation.

Neuenschwander, M. P., Düggeli, A., Nägele, C., \& Frey, S. (2015). Zweiter Zwischenbericht: Berufliche Entscheidungen und Entwicklungsverläufe im Jugendalter und jungen Erwachsenenalter (BEN). Solothurn, CH: Fachhochschule Nordwestschweiz, Pädagogische Hochschule, Institut Forschung und Entwicklung, Zentrum Lernen und Sozialisation.

Neuenschwander, M. P., Gerber, M., Frank, N., \& Rottermann, B. (2012). Schule und Beruf: Wege in die Erwerbstätigkeit. Wiesbaden, D: VS Verlag für Sozialwissenschaften.

Neuenschwander, M. P., Gerber, M., Frank, N., Singer, A., \& Bosshard, S. (2013a). (t1 - t5 L) Sozialisationsprozesse beim Übergang in den Lehrbetrieb (SoLe). Dokumentation der Lernendenbefragung. Zwischenerhebungen. Solothurn, $\mathrm{CH}$ : Pädagogische Hochschule der Fachhochschule Nordwestschweiz, Institut Forschung und Entwicklung, Zentrum Lernen und Sozialisation.

Neuenschwander, M. P., Hermann, M., Frank, N., \& Faschinger, S. (2013b). (W1 K4) Determinanten von Berufsbildungsentscheidungen beim Übergang in den Arbeitsmarkt (Ben). Dokumentation der Kohorte 4. Solothurn, CH: Fachhochschule Nordwestschweiz, Pädagogische Hochschule, Institut Forschung und Entwicklung, Zentrum Lernen und Sozialisation.

Neuenschwander, M. P., Nägele, C., \& Frey, S. (2016). Motivation to Participate in Formal Education during the School to Work Transition. Paper presented at the European Conference on Educational Research (ECER), 22.-26.8.2016, Dublin.

Nübling, M., Stößel, U., Hasselhorn, H. M., Michaelis, M., \& Hofmann, F. (2005). Methoden zur Erfassung psychischer Belastungen. Erprobung eines Messinstrumentes (COPSOQ). Dortmund, D, Berlin, D, Dresden, D: Bundesagentur für Arbeitsschutz und Arbeitsmedizin.

Parsons, F. (1909). Choosing a vocation. Boston, MA: Houghton Mifflin Company.

Savickas, M. L. (2012). Life design: A paradigm for career intervention in the 21 st century. Journal of Counseling \& Development, 90, 13-19.

Stalder, B. E., \& Nägele, C. (2015). Berufliche Identität, Commitment und Engagement. In M. Fischer, F. Rauner, \& Z. Zhao (Hrsg.), Kompetenzdiagnostik in der beruflichen Bildung - Methoden zum Erfassen und Entwickeln beruflicher Kompetenz (S. 259-273). Münster: LIT Verlag. 
Stalder, B. E., \& Schmid, E. (2016). Lehrvertragsauflösung und Ausbildungserfolg - kein Widerspruch. Bern, $\mathrm{CH}$ : hep verlag.

Super, D. E. (1980). A life-span, life-space approach to career development. Journal of Vocational Behavior, 16, 282-298.

Taris, T. W., Feij, J. A., \& Capel, S. (2006). Great expectations - and what comes of it: The effects of unmet expectations on work motivation and outcomes among newcomers. International Journal of Selection and Assessment, 14, 256-268.

Tims, M., Bakker, A. B., \& Derks, D. (2012). Development and validation of the job crafting scale. Journal of Vocational Behavior, 80(1), 173-186. http://doi.org/10.1016/j.jvb.2011.05.009

Trede, I. (2016). Laufbahnentscheidungen von Fachfrauen und Fachmännern Gesundheit nach Abschluss Ihrer Beruflichen Grundbildung. Bremen, D: Dissertation Universität Bremen.

Open Access Dieses Kapitel wird unter der Creative Commons Namensnennung 4.0 International Lizenz (http://creativecommons.org/licenses/by/4.0/deed.de) veröffentlicht, welche die Nutzung, Vervielfältigung, Bearbeitung, Verbreitung und Wiedergabe in jeglichem Medium und Format erlaubt, sofern Sie den/die ursprünglichen Autor(en) und die Quelle ordnungsgemäß nennen, einen Link zur Creative Commons Lizenz beifügen und angeben, ob Änderungen vorgenommen wurden.

Die in diesem Kapitel enthaltenen Bilder und sonstiges Drittmaterial unterliegen ebenfalls der genannten Creative Commons Lizenz, sofern sich aus der Abbildungslegende nichts anderes ergibt. Sofern das betreffende Material nicht unter der genannten Creative Commons Lizenz steht und die betreffende Handlung nicht nach gesetzlichen Vorschriften erlaubt ist, ist für die oben aufgeführten Weiterverwendungen des Materials die Einwilligung des jeweiligen Rechteinhabers einzuholen.

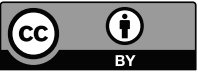


E

Ausblick 


\section{Hoffen auf Passung? \\ Homogenitätsfiktion und ihre Folgen}

Sandra Hupka-Brunner

\section{Zusammenfassung}

Zentral im Schweizerischen Bildungssystem ist der Glaube an die Wirksamkeit homogener Leistungsgruppen. Mit der Konstruktion leistungshomogener Gruppen (Selektion in Schultypen auf Sekundarstufe I oder durch pädagogische Massnahmen wie Klassenwiederholungen, Sonderbeschulung etc.) soll eine bessere Passung und dadurch eine erhöhte Motivation sowie bessere Leistungsentwicklung erreicht werden. Allerdings zeigt sich, dass diese pädagogische Hoffnung auf verschiedenen Ebenen (Leistung, Motivation, soziale Integration in die Klasse) enttäuscht wird. Die äussere Struktur scheinbar homogenisierter Lerngruppen scheint nicht automatisch dazu zu führen, dass die Jugendlichen eine erhöhte Passung wahrnehmen oder eine bessere Leistungsentwicklung erfahren. Die Tatsache, dass viele der Selektionsentscheide von der sozialen Herkunft der Betroffenen geprägt sind, wird anscheinend von vielen Akteuren und Akteurinnen im Bildungssystem ausgeblendet. Bildungspolitisch wird versucht, soziale Selektivität mittels Durchlässigkeit des Bildungssystems abzufedern. Es stellt sich aber die Frage, wie zielführend und effizient solche Massnahmen sind. Des Weiteren stellt sich die Frage, wie ein besserer Transfer wissenschaftlicher Ergebnisse in die Bildungspolitik und -praxis erfolgen kann. 


\section{$1 \quad$ Bildung im Lebensverlauf: Gesellschaftspolitische Zielsetzungen fürs Bildungssystem und wissenschaftliche Begleitung von Bildungsprozessen}

Bildungsverläufe von der Einschulung bis in die Erwerbstätigkeit - das vorliegende Buch umfasst eine lange Zeitspanne. Ziel des Buches ist es, diese unter einem psychologisch-erziehungswissenschaftlichen Blickwinkel zu untersuchen. Dafür entwickeln die Autoren einen Theorierahmen (Neuenschwander sowie Nägele und Stalder in diesem Buch), der sich für das Individuum in seinem Kontext interessiert und danach fragt, welchen Handlungsspielraum Menschen in einem gegebenen Kontext haben und wie sind in der Lage sind, sich immer wieder neuen Kontexten anzupassen. Bei diesem Passungs-Konzept wird davon ausgegangen, dass „sich Menschen optimal entwickeln, maximale Leistungen erbringen und zufrieden sind, wenn sie eine möglichst hohe Passung zwischen den individuellen Bedürfnissen und Zielen und den Anforderungen und Angeboten des Kontextes schaffen" (Neuenschwander in diesem Buch, S. 6).

Diese theoretischen Vorüberlegungen dienen während der weiteren Lektüre als roter Faden und verbinden somit die einzelnen Beiträge über die biographische Spanne hinweg. Insofern ist der theoretische Rahmen überzeugend und hilfreich. Dass potenziell stigmatisierende Konsequenzen bestimmter Ereignisse weniger ausführlich diskutiert werden, entspricht dem Anspruch und der Schwerpunktsetzung des Buches. Diese Aspekte werden im vorliegenden Beitrag punktuell ergänzt, wo es analytisch besonders sinnvoll erscheint.

Bildung wird als lebenslanger Prozess verstanden und schulische Bildung als Vorbereitung für das Leben konzipiert. Sie steht oftmals im Zentrum der Debatten, weil sie verschiedene, $z$. T. konfligierende Funktionen hat (Fend, 1981), die für eine Gesellschaft zentral sind: Die obligatorische Schule soll junge Menschen nicht nur fachlich qualifizieren, sondern sie auch auf ein Leben in einer demokratischen Gesellschaft vorbereiten (Integrations-/Akkulturationsfunktion). Darüber hinaus hat Schule eine Allokations- bzw. Selektionsfunktion, mit der sie zur gesellschaftlichen Platzierung der jungen Menschen beiträgt, indem sie Noten und Bildungszertifikate erteilt, die ihrerseits wiederum eine Berechtigung zu weiterführenden Studien implizieren oder den abnehmenden Institutionen (v. a. Lehrbetriebe, Gymnasien) eine Information über die schulische Leistungsfähigkeit der Schülerinnen liefern sollen.

Bereits in den 1960/70er Jahren wurden Bildungsdebatten unter dem Aspekt der Chancengleichheit geführt (Wenzel, 2010). Auch im Anschluss an PISA ${ }^{1}$ sind

1 Programme for International Student Assessment, siehe auch http://www.oecd.org/ berlin/themen/pisa-studie/ 
Bildungsdiskussionen durch ein Ringen um soziale Gerechtigkeit im Bildungssystem gekennzeichnet (OECD, 2002). Ebenso prägt die Sorge um die bessere „Ausschöpfung von Humanressourcen" bzw. um einen sich zuspitzenden Fachkräftemangel die aktuellen Debatten (EDK \& WBF, 2015; Sheldon, 2008). Dabei wurde und wird vor allem eine erhöhte Durchlässigkeit des Bildungssystems angestrebt (EDK \& WBF, 2015), um eine zu frühe Festlegung des Individuums auf einen einmal beschrittenen Bildungsweg zu verhindern und diesen möglichst lange flexibel (um)gestalten zu können ${ }^{2}$ (SKBF, 2014). Damit verbindet sich vor allem auch die Hoffnung auf ein höheres Mass an Chancengleichheit im Bildungssystem.

Vor diesem Hintergrund kommt längsschnittlichen Analysen von Bildungswegen besondere Bedeutung zu, denn nur mit ihnen ist es möglich, längerfristige Wirkungen von Bildungsstrukturen auf Bildungsverläufe zu untersuchen.

Im Beitrag von Moser, Oostlander und Tomasik (in diesem Buch) werden verschiedene Mechanismen genannt, die zur Entstehung von sozialer Ungleichheit beitragen: Zum einen wird das Entstehen von sozialen Ungleichheiten in Bildungsprozessen durch unterschiedliches Entscheidungsverhalten erklärt (Boudon, 1974; Breen \& Goldthorpe, 1997), zum anderen werden habituelle und ressourcenorientierte Ansätze zur Erklärung herangezogen (Bourdieu \& Passeron, 1971; Kramer \& Helsper, 2010). Moser et al. (ebd.) nennen des Weiteren die sogenannten Sommerloch-Studien, die zeigen, dass sich Leistungsdisparitäten zwischen sozialen Schichten während der Sommerferien erhöhen (Alexander, Entwisle, \& Olson, 2001; Verachtert, van Damme, Onghena, \& Ghesquière, 2009). Daraus folgern die Autoren, dass die Prozesse in der Schule eher disparitätsmindernde Eigenschaften hätten, die während der Sommerferien ausgesetzt werden, so dass schichtspezifische Unterschiede verstärkt zum Tragen kommen. Für Schulsysteme, in denen bereits früh versucht wird, leistungshomogene Schultypen („tracks“) einzuführen, ist darüber hinaus die Forschung zu spezifischen Entwicklungsmilieus interessant (Baumert, Stanat, \& Watermann, 2006; Moser, 2008). In solchen Schulsystemen (Deutschland und die Schweiz gelten als Systeme mit frühem und starkem Tracking) wird davon ausgegangen, dass der Unterricht in relativ homogenen Leistungsgruppen besser auf Voraussetzungen und Bedürfnisse der Schülerinnen und Schüler abgestimmt werden könne und dass vor allem schulisch schwache Schülerinnen und Schüler besser gefördert werden könnten. Dieser Gedanke entspricht in hohem Masse dem Konzept der Passung, das im vorliegenden Buch als Kerngedanke der theoretischen

2 Besonders deutlich wird das Bemühen um Durchlässigkeit am Slogan der Berufsbildungsreform 2004: „Kein Abschluss ohne Anschluss!“, für einen Überblick siehe auch https://www.sbfi.admin.ch/sbfi/de/home/themen/berufsbildung/berufsbildungsgesetz/ das-berufsbildungsgesetz--flexibel-fuer-neue-beduerfnisse.html Zugriff am 16.2.2017 
Rahmung anzusehen ist. Da Schulleistungen am Ende der Primarschulzeit aber mit der sozialen Herkunft der Kinder korrelieren, verbindet sich mit der Verteilung von Schülerinnen und Schülern auf institutionell getrennte Bildungsgänge immer auch eine soziale Trennung (Baumert et al., 2006). Baumert et al. (ebd.) betonen daher, dass soziale Segregation, verstanden als Trennung entlang der sozialen Herkunft der Kinder, als Kehrseite institutioneller Leistungsdifferenzierung zu sehen ist.

\section{Bildungsverläufe in der Schweiz - empirische Befunde}

Im Zentrum dieses Buches stehen Bildungsverläufe in der Schweiz. Das Schweizer Bildungssystem ist durch eine stark föderale Struktur geprägt (Volksschule ist Hoheitsbereich der Kantone), ein frühes und ausgeprägtes Tracking der Sekundarstufe I (Selektion von Schülerinnen und Schülern in möglichst leistungshomogene Schultypen nach der Primarschule) sowie eine Dominanz der dualen Berufsbildung auf Sekundarstufe II.

\subsection{Leistungshomogenität und Abweichungen von der Norm}

Bless (in diesem Buch) setzt bei der Leistungshomogenität an, deren Konstruktion durch verschiedene Selektionsmechanismen angestrebt wird. Damit verbindet sich die pädagogische Hoffnung, dass mit einer erhöhten Passung von Lernkontext und individuellen Möglichkeiten eine optimale Förderung aller Schülerinnen und Schüler (im Sinne einer besseren Leistungsentwicklung) zu verwirklichen ist. Dieser Glaube an die positiven Wirkungen solcher Massnahmen scheint bei vielen Akteuren im Schulsystem sehr ausgeprägt zu sein (Hofstetter, 2017).

\subsubsection{Nicht-intendierte Folgen pädagogischer Massnahmen}

Neben der Zuteilung auf verschiedene Schultypen seien auch Sonderbeschulungen sowie Klassenwiederholungen typische Massnahmen zur Homogenisierung der Lerngruppen. Bless (in diesem Buch) betont, dass viele dieser (gut gemeinten) Selektionsmassnahmen auch nicht-intendierte Nebenwirkungen haben. So zeigt er z.B. auf, dass für das Ziel einer möglichst leistungshomogenen Gruppe andere bedeutsame Heterogenitäten, z. B. hinsichtlich des Alters, in Kauf genommen werden (Bless in diesem Buch, Tabelle 1). Für die betroffenen Schülerinnen und Schüler muss allerdings davon ausgegangen werden, dass ein Abweichen vom erwarteten 
Alter wiederum z. B. bei der Lehrstellensuche negative Folgen haben dürfte (Imdorf, 2011), was seines Erachtens eher selten problematisiert werde. Wenn sich also mit pädagogischen Selektionsmassnahmen negative Folgen für die Schülerinnen und Schüler verbinden, muss geprüft werden, inwiefern die intendierte Wirkung erreicht wird. Der diesbezügliche Forschungsüberblick, den Bless (in diesem Buch) gibt, stimmt nachdenklich: So attestierte die Integrationsforschung der Aussonderung, insbesondere jener von Kindern mit Lernschwierigkeiten, eine geringe Effizienz. Zur Wirksamkeit der Typenwechsel auf der Sekundarstufe I sowie zur verspäteten Einschulung lägen für die Schweiz keine systematischen Untersuchungen vor. Hinsichtlich der pädagogischen Wirksamkeit von Klassenwiederholungen scheint sich aber zu zeigen, dass auch ihr Erfolg eher begrenzt ist: So werden zwar im ersten Wiederholungsjahr Leistungsverbesserungen erzielt, doch fallen viele der Repetierenden im zweiten Jahr nach der Repetition wieder zurück. Eine Klassenwiederholung scheint also nur kurzfristig eine positive Wirkung zu haben. Die damit verbundene Abweichung vom „Normverlauf“ bleibt jedoch bestehen und ist längerfristig sichtbar. Bless (in diesem Buch) bezeichnet die Langzeitwirkungen der Klassenwiederholungen als besorgniserregend, weil sie als Prädiktor für einen späteren negativen Verlauf der Schulkarriere (hohe Dropout-Rate, erhöhte Selektion in Schultypen mit geringerem Anspruchsniveau sowie Sonderschulung) gelten kann.

Warum sich Massnahmen ${ }^{3}$, die zu einem von der Norm abweichenden Verlauf führen, als problematisch erweisen, haben Gomolla \& Radtke (2002) für Deutschland untersucht. Sie konnten nachzeichnen, dass das Vorhandensein früherer Normabweichungen häufig als Argument für weitere Massnahmen verwendet wird. Dabei wird die "frühe Bedürftigkeit" eines Schülers oder einer Schülerin als Zeichen dafür gedeutet, dass auch zukünftig mit (Lern-)Problemen zu rechnen ist. Die Fördermassnahme (Qualifikationsfunktion der Schule) verliert an Wirkung, durch die Sichtbarkeit der Massnahme fliesst diese aber trotzdem in spätere Selektionsentscheide ein und trägt somit zur Erfüllung der Allokationsfunktion des Schulwesens bei. Hier zeigt sich, inwiefern pädagogisch gut gemeinte Massnahmen durch längerfristige nicht intendierte Nebenfolgen korrumpiert werden können. Dabei zeigt Bless (in diesem Buch) anhand der Analyse (z. T. kantonaler) schulstatistischer Daten, dass bis zum Ende der obligatorischen Schulzeit lediglich rund $50 \%$ der Jugendlichen eine „Normal-Schullaufbahn“ durchlaufen haben. Insofern müssen solche Phänomene ernst genommen werden. Bless (in diesem Buch) betont zudem, dass ein grosser Bedarf an längsschnittlichen Daten besteht, weil nur durch die Analyse von Längsschnittdaten das ganze Ausmass pädagogischer Massnahmen ersichtlich wird. Relevant ist auch, dass die Aussonderungsquoten

3 Z.B. Rückstellungen, Klassenwiederholungen, sonderpädagogische Massnahmen 
nicht zufällig erscheinen, sondern dass bestimmte Gruppen systematisch benachteiligt sind (Jugendliche mit Migrationshintergrund, Jungen). Dies verdeutlicht, dass neben Leistungsaspekten auch andere Faktoren ${ }^{4}$ dafür entscheidend sind, ob ein Kind pädagogische Sonderbehandlungen erfährt oder nicht.

\subsubsection{Prävention statt Problemzentriertheit?}

Ein interessantes Gegenbeispiel ist der FOKUS-Ansatz, dessen Evaluations-Ergebnisse in diesem Buch dargestellt werden (Benini et al. in diesem Buch). Ausgehend von der Erkenntnis, dass sich Unaufmerksamkeit, Hyperaktivität und Impulsivität als Indikatoren für einen erschwerten Bildungsverlauf erwiesen haben, versucht der FOKUS-Ansatz den schulischen Umgang mit diesen Phänomenen zu verbessern, indem u.a. an der Wahrnehmung der Lehrerinnen und Lehrer gearbeitet und z. B. auch für die Wirkung selbsterfüllender Prognosen sensibilisiert wird. Dieses pädagogische Massnahmenbündel unterscheidet sich von den bei Bless (in diesem Buch) diskutierten Massnahmen insofern, als es sich beim FOKUS-Ansatz um eine präventive Massnahme handelt, die ansetzt, bevor sich ein Problem verfestigt hat. Insofern verbindet sich damit auch keine Aussonderung der betroffenen Schülerinnen und Schüler. Stattdessen wird der Fokus auf verschiedene Interaktionsebenen gelegt, die normale Schulsituation (gleiche Klasse, selbe Lehrperson) aber beibehalten. Im Rahmen von Weiterbildungsveranstaltungen wurde an einer wirksamen Klassenführung (Klassenebene), dem Umgang mit einzelnen unaufmerksamen, hyperaktiven und impulsiven Kindern (individuelle Ebene) sowie der Zusammenarbeit mit den Eltern dieser Kinder gearbeitet. Der Vergleich mit einer entsprechenden Kontrollgruppe zeigt, dass sich nicht nur die Wahrnehmung der Lehrpersonen veränderte. Die meisten von ihnen wendeten die in der Weiterbildung erlernten Methoden im Unterricht an, was einen deutlichen positiven Einfluss auf die betroffenen Schüler hatte.

4 Vgl. z. B. Bless in diesem Buch: biologische Fragilität des männlichen Geschlechts, extrovertiertes Verhalten der Knaben und höhere Leistungserwartungen an sie. Bezüglich Migrationshintergrund: mangelnde Kenntnisse der Unterrichtssprache, kulturelle Eigenheiten, mangelnde familiäre Unterstützungsmöglichkeiten, niedriger sozioökonomischer Status, ungenügende Kenntnisse des Bildungssystems und Unterschätzung der Schulleistungen durch die Lehrpersonen. Bei Hofstetter (2017) sowie Kronig (2007) finden sich Hinweise, dass die regionale Organisation der Schulstrukturen die Bildungschancen der Kinder und Jugendlichen massgeblich beeinflusst. 


\subsubsection{Die Rolle der Wahrnehmung in pädagogischen Prozessen}

Wie wichtig die Wahrnehmung der Kinder (und ihrer Familien) durch die Lehrerinnen und Lehrer für die schulische Laufbahn sein kann, zeigt auch der Beitrag von Neuenschwander und Niederbacher (in diesem Buch). Demnach haben Lehrpersonen bestimmte Leistungserwartungen an Schülerinnen und Schüler, die u. a. auch von der sozialen Herkunft der Kinder und Jugendlichen beeinflusst werden. Dies ist insofern bedeutsam, als dass die Lehrererwartung an sich die Leistungsentwicklung der Kinder und Jugendlichen beeinflusst (Pygmalion-Effekt). Dabei können Leistungserwartungen implizit oder explizit sein und mehr oder weniger deutlich kommuniziert werden. Der Beitrag zeigt, dass die Lehrpersonen bereits zwei Jahre vor dem Übergang in die Sekundarstufe I eine Selektions-Erwartung haben $^{5}$, die auf einer impliziten Beurteilung der schulischen Leistungen und der Intelligenz sowie der familiären Unterstützung und der Erwartungen der Eltern an ihre Kinder beruht. Diese Erwartung wird nicht explizit kommuniziert, beeinflusst aber trotzdem die Leistungsentwicklung der nächsten zwei Jahre, die dann zum entscheidenden Kriterium für den Übergang in die Sekundarstufe I wird. Zudem kommunizieren Lehrerinnen und Lehrer kurz vor dem Übertritt in die Sekundarstufe I ihre Schulniveau-Erwartung („Übertrittsempfehlung“), was für Kinder eine sehr deutliche Form der Leistungsrückmeldungen darstellt. Konsequenterweise beeinflusst diese kommunizierte Leistungsprognose den weiteren Lernverlauf der Schülerinnen und Schüler (Neuenschwander und Niederbacher in diesem Buch). Lehrererwartungen beeinflussen also direkt und indirekt die Allokation auf die unterschiedlichen Leistungszüge der Sekundarstufe I.

Hinzu kommt, dass Kinder nicht als „unbeschriebene Blätter“ in die Schule kommen und sich dann gemäss Begabung, Fleiss und Unterrichtskonzeption entwickeln. Vielmehr unterscheiden sie sich (schichtspezifisch) bereits vor Schulbeginn hinsichtlich ihrer Kompetenzen (Moser et al. in diesem Buch) ${ }^{6}$. Die Autoren (ebd.) konnten nachzeichnen, dass die Primaschule nicht in der Lage ist, Anfangsunterschiede auszugleichen, sondern dass sich diese Disparitäten in den ersten drei Jahren sogar verstärken. Dabei gehen sie davon aus, dass die soziale Herkunft für

5 Zu ähnlichen Befunden kommt auch Hofstetter (2017).

6 Gemäss Angelone et al. (2012) sind soziale Herkunft, Geschlecht und fachspezifisches Vorwissen vor Schuleintritt Faktoren, die die Leistungsentwicklung in der Primarschule erklären. Für einen Überblick über den internationalen Forschungsstand zur Leistungsentwicklung in verschiedenen Schulmodellen siehe auch (Moser, 2008). 
die Leistungsentwicklung und den Übertritt ins Langzeitgymnasium ${ }^{7}$ besonders wichtig ist.

\subsection{Homogenitätsfiktion und Passung?}

Die Einteilung von Schülerinnen und Schülern in verschiedene, möglichst leistungshomogene Gruppen („tracks") im Anschluss an die Primarschule stellt die verbreitetste Massnahme zur Homogenisierung der Schülerschaft dar. Doch wie erfolgt die Zuteilung zu den verschiedenen Schultypen respektive Anforderungsniveaus?

\subsubsection{Selektionsmechanismen: Konstruktion von leistungshomogenen Lerngruppen auf Sekundarstufe I?}

Wenn auf verschiedene Schultypen selektioniert wird, um möglichst leistungshomogene Klassen zu schaffen, sollten Leistungsmerkmale das ausschlaggebende Selektions-Kriterium sein. Moser et al. (in diesem Buch) zeigen aber, dass Schulleistungen stark von der sozialen Herkunft abhängig sind und dass die Primarschule kaum kompensatorisch zu wirken scheint. Neuenschwander und Niederbacher (in diesem Buch) machen ihrerseits deutlich, dass Leistungen der Jugendlichen durch die Erwartungen der Lehrkräfte geprägt werden. Auch die Allokation in verschiedene Schultypen erfolgt nicht rein leistungsbasiert. So konnte z. B. Neuenschwander (2010) für die Deutschschweiz zeigen, dass je nach Struktur der Sekundarstufe I (Anzahl und Grösse der verschiedenen Schultypen) eine gruppenspezifische Benachteiligung unterschiedlichen Ausmasses nachweisbar ist, die allein aufgrund von Leistungskriterien nicht erklärt werden kann. Auch ein früherer Zeitpunkt des Übertritts in die Sekundarstufe I scheint mit einer höheren sozialen Selektivität einher zu gehen (Berger \& Combet, 2016; Büchler, 2016). Bis vor kurzem traten die Schülerinnen und Schüler in der Schweiz nach fünf oder sechs Jahren Primarschule in die Sekundarschule I über ${ }^{8}$. Des Weiteren existieren Bezugsgruppeneffekte, die dazu führen, dass je nach Zusammensetzung der Klasse Schülerinnen und Schüler bei gleichem Leistungsstand unterschiedliche Chancen auf eine bestimmte Schultyp-Empfehlung hatten (Gröhlich \& Guill, 2009; Trautwein \& Baeriswyl, 2007). Ein Blick in die verschiedenen kantonalen Übertrittsregelungen

7 Im Kanton Zürich besteht die Wahl zwischen dem Langgymnasium nach sechs Jahren Primarschule oder dem Kurzgymnasium ab der 2. oder 3. Sekundarklasse.

8 Mittlerweise wurde dies angeglichen, so dass in allen Kantonen (mit Ausnahme des Tessins) der Übertritt nach sechs Jahren erfolgt. 
zeigt, dass neben den Leistungsbeurteilungen auch das Arbeitsverhalten oder der Übertrittswunsch der Eltern bzw. der Kinder in den Entscheidungsprozess mit einfliesst. Letztere werden hinzugezogen, um eine möglichst präzise Prognose der Leistungsentwicklung zu treffen. Zudem scheinen Lehrerinnen und Lehrer bestrebt zu sein, Kinder nicht durch zu ambitiöse Schultyp-Empfehlungen zu überfordern (Hofstetter, 2017). Insbesondere die erwartete Elternunterstützung scheint aber mit dafür verantwortlich zu sein, dass dieser Übergang hoch sozial selektiv ist (Büchler, 2016; Neugebauer, 2010). Dabei kann die prognostische Qualität der Empfehlungen in Zweifel gezogen werden (Baeriswyl, Trautwein, Wandeler, \& Lüdtke, 2009; Schuchart \& Weishaupt, 2004). Gemäss Hofstetter (2017) glauben Deutschfreiburger Lehrerinnen und Lehrer in starkem Masse an die Wirksamkeit und Gerechtigkeit eines fairen Selektionsverfahrens. Sie bemühen sich sehr, den verschiedenen Anforderungen an ihren Beruf (Selektion vs. Förderung) gerecht zu werden (Streckeisen, Hänzi, \& Hungerbühler, 2007). Dabei deuten sie Misserfolge der Kinder oft in Begabungsdefizite oder mangelnde Anstrengungsbereitschaft um, ohne dabei die Umstände des Zustandekommens dieses Misserfolgs zu hinterfragen. Zentral erscheint dabei die „Prä-Kategorisierung“ der Schülerinnen und Schüler über Noten, wobei in Zweifelsfällen der soziale Vergleich innerhalb der Klasse mit beachtet wird. Hält man sich vor Augen (Kronig, 2007), wie stark auch Noten von der sozialen Herkunft geprägt sind, wird deutlich, in welch starkem Ausmass die soziale Ungleichheit die Entscheidungspraktiken der verschiedenen Akteure direkt und indirekt prägt.

Die vorhergehenden Abschnitte haben gezeigt, dass diese Selektion stark von der sozialen Herkunft geprägt ist, aber in individuelle Leistungen bzw. Leistungsdefizite umgedeutet wird. Dass sich die pädagogische Hoffnung auf eine „passende“ Förderung und geringere Überforderung der Schülerinnen und Schüler in den verschiedenen Schultypen nicht immer erfüllt, kann anhand der folgenden Beiträge dieses Buches nachgezeichnet werden.

\subsubsection{Bessere Passung durch homogene Leistungsgruppen?}

Neuenschwander (in diesem Buch) kann zeigen, dass in der Schweiz die Passungswahrnehmung nach dem Übertritt in die Sekundarstufe I abnimmt. Dies ist insofern bemerkenswert, als die Selektion in verschiedene Schultypen ja gerade leistungshomogenisierend und damit passungserhöhend wirken sollte. Neuenschwander (ebd.) geht davon aus, dass strukturelle Aspekte des Unterrichts (kein Klassenlehrerprinzip mehr, grössere Gruppen) diesen Effekt verursachen.

Um sich diesen Fragen zu nähern, hat Neuenschwander (in diesem Buch) Schülerinnen und Schüler untersucht, die den Übertritt von der Primar- in die Sekundarschule zu unterschiedlichen Zeitpunkten (5. bzw. 6. Jahrgangsklasse) 
vollziehen. Der Vorteil dieses Untersuchungsdesigns ist, dass für Alterseffekte kontrolliert werden kann. Dabei zeigt sich, dass diejenigen Schülerinnen und Schüler, die zu einem späteren Zeitpunkt wechseln, in Deutsch und Mathematik höhere Leistungen erzielen und motivationale Aspekte (Lern-/Leistungszielorientierung) besser bleiben. Dank des Untersuchungsdesigns dieser Studie lässt sich dabei schlussfolgern, dass es sich hier nicht um Alterseffekte handelt, sondern eher um Effekte der institutionellen Strukturierung und der Lernpraxis im Unterricht (Fachunterricht, der weniger stark an die Fähigkeiten der Jugendlichen angepasst wird und meist stärker lehrerzentriert erfolgt). Angelone et al. (2013) berichten im Vergleich zur Primarschule einen geringeren Leistungszuwachs während der Sekundarstufe I und verstehen diesen als eine Folge der unterschiedlichen Struktur der Lehrpläne in der Primarschule im Vergleich zur Sekundarstufe I. Neuenschwander (in diesem Buch) interpretiert die Befunde dahin gehend, dass die Veränderung der Noten und der Lern- und Leistungszielorientierung mit der Abnahme der Passungswahrnehmung beim Übergang in die Sekundarstufe I erklärt werden kann. Er kommt zu dem Schluss: „Interessant ist, dass sich die Gruppierung der Kinder in leistungshomogene Schulklassen aufgrund der Einteilung in Schulniveaus nicht in einer höheren Passungswahrnehmung, Motivation und Leistungszunahme auswirkt. Die Hoffnung, die Lernbedingungen durch eine Leistungsgruppierung in Schulniveaus zu verbessern, wird nicht innerhalb von 1.5 Jahren nach Eintritt in die Sekundarstufe I erfüllt." (Neuenschwander in diesem Buch, S. 158/159).

Wenn also die Einteilung in vermeintlich leistungshomogene Gruppen allein nicht ausreicht, um die erwünschte Passung zu erhöhen, stellt sich die Frage, ob sich der enorme Aufwand lohnt, oder ob andere Massnahmen nicht besser geeignet wären.

Oder gibt es andere Aspekte im Rahmen des Trackings, von denen die Schülerinnen und Schüler profitieren können? Immerhin scheinen Lehrerinnen und Lehrer in ihre Übertrittsempfehlungen auch Aspekte wie Selbstkonzepte und Wohlbefinden (beispielhaft: geringeres Überlastungsempfinden) zu berücksichtigen.

Im Sekundarschulalter wächst die Bedeutung der Peers. Es kann angenommen werden, dass die Beliebtheit in der Klasse zum bedeutenden Faktor für das individuelle Wohlbefinden und die Leistungsentwicklung wird. Insofern ist es nahe liegend, auch die Beliebtheitsentwicklung von Jugendlichen in Abhängigkeit ihrer Schullaufbahn zu analysieren.

Rösselet und Neuenschwander (in diesem Buch) können zeigen, dass eine hohe Akzeptanz bei den Peers am Ende der Primarschule mit guten Leistungen, Noten und hohem sozialen Selbstkonzept einhergehen. Sie zeigen aber auch, dass der Übertritt in die Sekundarstufe I nicht nur die motivationale und Leistungsentwicklung tangiert, sondern auch die Integration und Beliebtheit in der Klasse. Dabei lockert sich am Anfang der Sekundarstufe I die Koppelung von Beliebtheit, 
Leistung und Selbstkonzepten. Insbesondere die Beliebtheit erweist sich als weniger stabil für (gleichaltrige) Schülerinnen und Schüler, die bereits die Transition in die Sekundarstufe I vollzogen haben, wobei das Schulniveau und die Leistungsposition in der Klasse (in Mathematik) die Beliebtheit beeinflussen. Hier könnte man schlussfolgern, dass schulisch schwache Schülerinnen und Schüler vom Tracking auf Sekundarstufe I profitieren, weil ihr Selbstwert und ihre fachlichen Selbstkonzepte durch die Neu-Positionierung in der Leistungshierarchie entlastet werden können. Diese potenzielle Entlastung geht allerdings mit diversen möglichen Negativ-Folgen (ungünstigere Entwicklungsmilieus, Pygmalion, geringere Leistungsentwicklung etc.) einher, wie folgendes Beispiel zeigt:

Meine Nachhilfeschülerin nahm während der Primarschule eine schlechte Position in der Leistungshierarchie ihrer Klasse ein und litt darunter. Ihre Lernmotivation war gering, da sie oft ihr schulisches Scheitern antizipierte. Nach dem Übergang in die Sekundarstufe I besuchte sie dann das niedrigste Schulniveau. Zu Beginn des neuen Schuljahres wurde Grundschulstoff repetiert, und sie stellte fest, dass sie nun zu den besseren Schülerinnen und Schülern gehörte. Die neue Position in der Leistungshierarchie steigerte zuerst ihr Wohlbefinden, führte aber zwischenzeitlich auch zu geringerer Leistungsmotivation, weil zusätzliche Lernanstrengungen nicht notwendig erschienen. Gegen Ende der obligatorischen Schulzeit aber überwog das Gefühl, „nur eine Hauptschülerin“ zu sein. Zu diesem Zeitpunkt waren weniger die klasseninternen Bezugsgruppenvergleiche für das eigene Selbstwertgefühl und die fachlichen Selbstkonzepte ausschlaggebend, sondern vielmehr die Vergleiche mit anderen Schultypen. Zudem war das Bewusstsein für das schlechte Image eines Hauptschulabschlusses sehr ausgeprägt.

Zusammenfassend scheint es, als würde ein Grossteil der pädagogischen Hoffnungen, die sich an eine erhöhte Passung qua Selektion auf verschiedene Schultypen knüpfen, enttäuscht. Dafür muss mit nicht-intendierten Nebenfolgen und längerfristigen negativen Konsequenzen gerechnet werden.

\subsection{Nachobligatorische Bildungsverläufe}

Wie gestalten sich Bildungsverläufe nach der obligatorischen Schulzeit? Welche Rolle spielen der Schultyp und das Passungskonzept für nachgelagerte Bildungsaktivitäten?

\subsubsection{Lieber ein guter Realschüler als ein schlechter Sekundarschüler?}

Die Zuteilung zu leistungsgetrennten Schultypen entspricht dem gesellschaftlichen Auftrag der Schule. Wie eingangs erläutert soll der Schultyp dabei - ebenso 
wie die Noten - den abnehmenden Bildungsinstitutionen Informationen über die Leistungsfähigkeit der Schülerinnen und Schüler zur Verfügung stellen.

Konsequenterweise erweist sich der Schultyp der Sekundarstufe I als eines der zentralen Selektionskriterien der abnehmenden Institutionen auf Sekundarstufe II. Im Bereich der Berufsbildung sind Betriebe besonders bei hohem Bewerberandrang darauf angewiesen, auf Basis möglichst gut legitimierbarer Selektionskriterien eine Auswahl zu treffen. Neben den Noten ist daher der auf Sekundarstufe I besuchte Schultyp eines der wichtigsten Auswahlkriterien - selbst wenn die Aussagekraft des Schultyps als Leistungsindikator sehr unzuverlässig ist. Die Bedeutsamkeit des Einflusses des Schultyps auf die Lehrlingsselektion ist vielfach belegt (Häfeli, Neuenschwander, \& Schumann, 2015; Hupka-Brunner, Sacchi, \& Stalder, 2011; Imdorf, 2007; Moser, 2004; Sacchi, Hupka-Brunner, Stalder, \& Gangl, 2011).

Die z.T. gravierenden Probleme, mit denen sich Jugendliche bei der Lehrstellensuche konfrontiert sehen, sind mit ein Grund, warum sich in den letzten zwanzig Jahren Brückenangebote etabliert haben (Hupka-Brunner, Meyer, Stalder, \& Keller, 2011).

\subsubsection{Folgen früher Selektion: Durchlässigkeit und Weiterbildungen als mögliches Korrektiv?}

Diesem Thema widmet sich Düggeli (in diesem Buch), wenn er direkte und indirekte Übertritte in Basel untersucht und den ursprünglichen Aspirationen der Jugendlichen gegenüberstellt. Dabei thematisiert er, wie bedeutend der lokale Kontext ist, in dem die Jugendlichen sich zu orientieren haben, da die lokalen Strukturen die Optionen und damit auch ihre Wahlmöglichkeiten definieren. Düggeli folgert, dass es eine vorrangige Aufgabe der Berufswahlbegleitung sei, mit den Jugendlichen gemeinsam zu bestimmen, wann es sinnvoll sein könnte, von der ursprünglich angestrebten Lösung abzuweichen, und wann nicht. Im Zentrum der Überlegungen steht die Idee der möglichen Kompensation allfälliger Defizite. Dabei sei nicht nur die angenommene Erfolgswahrscheinlichkeit bedeutsam, sondern auch die subjektive Bedeutung eines Scheiterns. Die grosse Bedeutung des räumlich-institutionellen Kontextes für den Übergang in die Sekundarstufe II konnte in letzter Zeit mehrfach belegt werden (Glauser \& Becker, 2016; Sacchi \& Meyer, 2016). Neben diesem seien auch die soziale Herkunft, der absolvierte Schultyp, berufliche Orientierungsschwierigkeiten sowie die (familiäre und schulische) Unterstützung sowie das Geschlecht entscheidend dafür, ob eine Person ein Brückenangebot besuche oder nicht (Sacchi \& Meyer, 2016)2016. Sie gehen davon aus, dass nicht-kognitive Kompetenzen und motivationale Aspekte nur am Rande relevant seien, und dass sich für die Schulnoten und kognitiven Kompetenzen (PISA-Lesekompetenzen) kein eigenständiger Einfluss nachweisen lasse. Damit stehen ihre Befunde in einem 
deutlichen Gegensatz zu dem - auch von Düggeli kritisch thematisiertem - Defizit-Kompensations-Paradigma, welches die Gründe für einen verzögerten Einstieg in die Sekundarstufe II vor allem in Kompetenzdefiziten, ungünstigen Dispositionen und ungenügenden schulischen Leistungen der einzelnen Jugendlichen verortet.

Kompetenzdefizite können also nur zu einem geringen Anteil erklären, welche Jugendlichen ein Brückenangebot besuchen und welche nicht. Dies ist umso bedeutsamer, als der Besuch eines Brückenangebots (auch unter Kontrolle von Leistungsmerkmalen auf Sekundarstufe I) den weiteren Bildungsverlauf beeinflusst: Wie Scharenberg et al. (2016) zeigen konnten, erweisen sich Brückenangebote, aber auch der Besuch eines Schultyps mit Grundanforderungen, für alle Zertifikats-Typen auf Sekundarstufe II als wichtige Prädiktoren.

Angesichts der starken Folgewirkungen der frühen Selektion ist es wenig verwunderlich, dass die Bildungspolitik um erhöhte Durchlässigkeit bemüht ist. Insbesondere in der Berufsbildung wurden die Bestrebungen verstärkt, die Anschlussfähigkeit an Bildungsprogramme der nächsten Stufe zu verbessern („Kein Abschluss ohne Anschluss!").

Inwiefern diese Bestrebungen um eine erhöhte Durchlässigkeit allerdings wirklich in der Lage sind, Chancengerechtigkeit zu erhöhen, scheint fraglich. Zumindest zeigt die bisherige Forschung zur „Nachholenden Bildung“ (Schräder-Naef \& JörgFromm, 2005), dass nachträgliche Bildungskorrekturen sehr schwer zu realisieren sind. Dies kann damit erklärt werden, dass Ausbildungsverläufe, die - wie Bless in seinem Beitrag (in diesem Buch) verdeutlicht -zur Verlängerung der Bildungslaufbahn führen, Gefahr laufen, als normabweichend wahrgenommen und dadurch ein Stück weit entwertet zu werden, weil das Alter, in dem ein Zertifikat erworben wird, zum Stigma werden kann. Zudem benötigen diese Verläufe mehr Zeit und Ressourcen, die insbesondere benachteiligte Jugendliche nur selten zu mobilisieren im Stande sind. Ausserdem ist zu berücksichtigen, dass berufliche Lebenspläne oftmals mit anderen Lebensbereichen wie z. B. Familiengründung abgestimmt werden und sich gegenseitig bedingen, weil die individuellen Freiheitsgrade auch vom Grad der Verpflichtungen anderen gegenüber und/oder der erfahrenen Unterstützung abhängig sind.

Auch Weiterbildungen gelten als eine Massnahme, um Bildungsverläufe zu optimieren. Allerdings hängt die Teilnahme an Weiterbildungen stark von der zuvor erlangten Bildung sowie von externen Gelegenheitsstrukturen ${ }^{9} \mathrm{ab}^{10}$. Im Beitrag von

9 Angebot der Weiterbildungen, Unterstützung durch den Arbeitgeber etc.

10 „Weiterbildung führt also in der Tendenz nicht zu einem Ausgleich von sozialen Disparitäten, sondern zu zusätzlichen sozialen Ungleichheiten zwischen gering und gut qualifizierten Personen." (SKBF, 2014, S. 276) 
Nägele et al. (in diesem Buch) werden sie als eine mögliche Strategie von Individuen konzipiert, um die berufliche Passung zu erhöhen. Dabei wird zwar deutlich, dass die wahrgenommene Passungswahrnehmung einen sehr grossen Einfluss auf die Zufriedenheit und die Verbundenheit mit dem Beruf sowie die Planung von Weiterbildungstätigkeiten hat. Allerdings zeigt sich der Einfluss auf die geplanten Weiterbildungsaktivitäten vor allem für Personen, die eine sehr geringe Passung zu ihrem Beruf aufweisen. Damit erweist sich das Passungskonzept als zwar ein theoretischer Hintergrund, der auch in der Arbeitswelt erklärungswirksam ist. Allerdings scheint die Erklärungskraft dieses Ansatzes nicht für alle Gruppen zu gelten. Es stellt sich die Frage, inwiefern die zuvor angesprochene Verflechtung von verschiedenen Lebensbereichen, die damit verbundenen Ressourcen sowie die externen Gelegenheitsstrukturen nicht stärker zu berücksichtigen wären.

\section{Ausblick: Wissenschaftliche Erkenntnisse und pädagogische Praxis}

Das vorliegende Buch zeigt sehr überzeugend, dass das Passungskonzept eine wissenschaftlich ertragreiche Folie zur Analyse von Bildungswegen in der Schweiz darstellt: Es schärft den analytischen Blick, und die referierten Ergebnisse werfen weiterführende Fragen an die Bildungspraxis und Politik auf.

Es wird deutlich, dass das Schulsystem in der Schweiz mit einem starken Glauben daran arbeitet, dass leistungshomogene Gruppen chancengerecht sind: Zentral ist die Idee, dass leistungshomogene Gruppen eine höhere Passung von individuellen Fähigkeiten und Lernkontext ermöglichen, was insgesamt zu einer besseren Leistungsentwicklung führen sollte. Sie dienen also, so das vorherrschende Paradigma, der optimalen Förderung der Betroffenen und sind "chancenerhöhend“. Zudem zeigt sich bei den Akteuren des Systems die Überzeugung, dass die (homogenisierenden) Selektionspraktiken leistungsgerecht vollzogen würden, was empirisch so nicht haltbar ist.

Die Beiträge des vorliegenden Sammelbandes veranschaulichen sehr deutlich, dass diverse Aspekte (Leistungsentwicklung, Motivation, soziale Integration) dieser Hoffnung enttäuscht werden und dass allein die Selektion in verschiedene Gruppen anscheinend nicht zu einer ausreichenden Adaptivität der Lernumwelt an die Schülerinnen und Schüler führt. Dagegen mehren sich die Hinweise, dass mit dieser Einteilung negative Folgen verbunden sind, die aber nicht als „Kollateralschäden" der Selektionspraxis gedeutet werden, sondern als individuelles Defizit. Verschiedene pädagogische Massnahmen scheinen also entgegen ihrer Intention, 
Chancengleichheit nicht zu erhöhen, sondern im Gegenteil, soziale Ungerechtigkeiten im Bildungssystem zu verschärfen.

Auf bildungspolitischer Ebene wird das Prinzip der Durchlässigkeit des Bildungssystems als zentral erachtet, um frühere Bildungsentscheidungen auch nachträglich noch korrigieren zu können und Chancengleichheit zu gewährleisten. Dieses Vorgehen ist insofern sinnvoll und „pragmatisch“, als bei bestehenden Strukturen angesetzt wird. Hält man sich aber vor Augen, wie risikoreich, langwierig und schwierig unter Umständen die Korrekturen von Bildungswegen sind und wie bedeutsam das Auftreten, das Timing und die Dauer von Selektionsentscheiden für den weiteren Bildungsverlauf sein können, stellt sich die Frage, ob andere Massnahmen nicht effizienter wären.

Um dies zu entscheiden, bräuchte es neben einem intensiveren Dialog zwischen Wissenschaft und Bildungspraxis bzw. -politik vor allem vermehrt längsschnittliche Daten und Evaluationen von Massnahmen, um nicht nur kurzfristige Wirkungen, sondern auch längerfristige Folgen besser abschätzen zu können.

\section{Literatur}

Alexander, K. L., Entwisle, D. R., \& Olson, L. S. (2001). Schools, achievement, and inequality: A seasonal perspective. Educational Evaluation and Policy Analysis, 23, 171-191. doi:10.3102/01623737023002171

Angelone, D., Keller, F., \& Moser, U. (2013). Entwicklung schulischer Leistungen während der obligatorischen Schulzeit. Bericht zur vierten Zürcher Lernstandserhebung zuhanden der Bildungsdirektion des Kantons Zürich. Zürich: Universität Zürich.

Angelone, D., \& Ramseier, E. (2012). Die Kluft öffnet sich. Herkunftseffekte auf die schulischen Leistungen verstärken sich im Verlauf der Primarschule. Swiss Journal of Sociology, 38(2), 223-244.

Baeriswyl, F., Trautwein, U., Wandeler, C., \& Lüdtke, O. (2009). Wie gut prognostizieren subjektive Lehrerempfehlungen und schulische Testleistungen beim Übertritt die Mathematik- und Deutschleistung in der Sekundarstufe I? Zeitschrift für Erziehungswissenschaft, 12(7), 352-372.

Baumert, J., Stanat, P., \& Watermann, R. (2006). Schulstruktur und die Entstehung differenzieller Lern- und Entwicklungsmilieus. In J. Baumert, P. Stanat, \& R. Watermann (Hrsg.), Herkunftsbedingte Disparitäten im Bildungswesen: Differenzielle Bildungsprozesse und Probleme der Verteilungsgerechtigkeit (S.95-188). Wiesbaden: VS Verlag für Sozialwissenschaften.

Berger, J., \& Combet, B. (2016). Late school tracking, less class bias in educational decision-making? The uncertainty reduction mechanism and its experimental testing. European Sociological Review, 1-13. doi:10.1093/esr/jcw054 
Boudon, R. (1974). Education, opportunity, and social inequality. changing prospects in Western society. New York: John Wiley.

Bourdieu, P., \& Passeron, J.-C. (1971). Die Illusion der Chancengleichheit. Untersuchungen zur Soziologie des Bildungswesens am Beispiel Frankreichs. Stuttgart: Klett.

Breen, R., \& Goldthorpe, J. H. (1997). Explaining educational differentials: Towards a formal rational action theory. Rationality and Society, 9(3), 275-305.

Büchler, T. (2016). Schulstruktur und Bildungsungleichheit: Die Bedeutung von bundeslandspezifischen Unterschieden beim Übergang in die Sekundarstufe I für den Bildungserfolg. Kölner Zeitschrift für Soziologie und Sozialpsychologie, 68, 53-87. doi:10.1007/ s11577-015-0350-5

EDK, \& WBF (2015). Chancen optimal nutzen. Erklärung 2015 zu den gemeinsamen bildungspolitischen Zielen für den Bildungsraum Schweiz. Bern: EDK.

Fend, H. (1981). Theorie der Schule. München, Wien, Baltimore: Urban \& Schwarzenberg.

Glauser, D., \& Becker, R. (2016). VET or general education? Effects of regional opportunity structures on educational attainment in Germanspeaking Switzerland. Empirical Research in Vocational Education and Training, 8(8). doi:10.1186/s40461-016-0033-0

Gomolla, M., \& Radtke, F.-O. (2002). Institutionelle Diskriminierung. Die Herstellung ethnischer Differenz in der Schule. Opladen: Leske + Budrich.

Gröhlich, C., \& Guill, K. (2009). Wie stabil sind Bezugsgruppeneffekte der Grundschulempfehlung für die Schulformzugehörigkeit in der Sekundarstufe? Journal für Bildungsforschung Online, 1(1), 154-171.

Häfeli, H., Neuenschwander, M. P., \& Schumann, S. (2015). Berufliche Passagen im Lebenslauf. Berufsbildungs- und Transitionsforschung in der Schweiz. SpringerLink.com: Springer VS.

Hofstetter, D. (2017). Die schulische Selektion als soziale Praxis. Aushandlungen von Bildungsentscheidungen beim Übergang von der Primarschule in die Sekundarstufe I. Weinheim Basel: Beltz Juventa.

Hupka-Brunner, S., Meyer, T., Stalder, B. E., \& Keller, A. (2011). PISA-Kompetenzen und Übergangswege: Ergebnisse aus der Schweizer TREE-Studie. In E. M. Krekel \& T. Lex (Hrsg.), Neue Jugend, neue Ausbildung? Beiträge aus der Jugend- und Bildungsforschung (S. 173-188). Bonn: Bundesinstitut für Berufsbildung.

Hupka-Brunner, S., Sacchi, S., \& Stalder, B. (2011). Social origin and access to upper secondary education in Switzerland: A comparison of company-based apprenticeship and exclusively school based programmes. In M. M. Bergmann, S. Hupka-Brunner, A. Keller, T. Meyer, \& B. E. Stalder (Eds.), Transitionen im Jugendalter. Ergebnisse der Schweizer Längsschnittstudie TREE (Vol. 1, pp. 157-182). Zürich: Seismo.

Imdorf, C. (2007). Lehrlingsselektion in KMU. Kurzbericht März 2007. Freiburg (CH): Heilpädagogisches Institut der Universität Freiburg.

Imdorf, C. (2011). Zu jung oder zu alt für eine Lehre? Altersdiskriminierung bei der Ausbildungsplatzvergabe. Zeitschrift für Arbeitsmarktforschung, online first.

Kramer, R.-T., \& Helsper, W. (2010). Kulturelle Passung und Bildungsungleichheit - Potenziale einer an Bourdieu orientierten Analyse der Bildungsungleichheit. In H.-H. Krüger, U. Rabe-Kleberg, R.-T. Kramer, \& J. Budde (Hrsg.), Bildungsungleichheit revisited. Bildung und soziale Ungleichheit vom Kindergarten bis zur Hochschule (S. 103-125). Wiesbaden: VS Verlag für Sozialwissenschaften.

Kronig, W. (2007). Die systematische Zufälligkeit des Bildungserfolgs. Theoretische Erklärungen und empirische Untersuchungen zur Lernentwicklung und Leistungsbewertung in unterschiedlichen Schulklassen. Bern: Haupt. 
Moser, U. (2004). Jugendliche zwischen Schule und Berufsbildung. Eine Evaluation bei Schweizer Grossunternehmen unter Berücksichtigung des internationalen Schulleistungsvergleichs PISA. Bern: h.e.p. Verlag.

Moser, U. (2008). Schulsystemvergleich: Gelingensbedingungen für gute Schulleistungen. Expertise über die Bedeutung von Schulmodellen der Sekundarstufe I für die Entwicklung der Schulleistungen. Zürich: Universität Zürich, Institut für Bildungsevaluation.

Neuenschwander, M., \& Grunder, H.-U. (2010). Schulübergang und Selektion. Zürich: Rüegger Verlag.

Neugebauer, M. (2010). Bildungsungleichheit und Grundschulempfehlung beim Übergang auf das Gymnasium: Eine Dekomposition primärer und sekundärer Herkunftseffekte. Zeitschrift für Soziologie, 39(3), 202-214.

OECD (2002). Qualität und Chancengleichheit verbessern: Ergebnisse aus PISA2000. In OECD (Hrsg.), Bildungspolitische Analyse 2002 (S. 41-73). Paris: OECD.

Sacchi, S., Hupka-Brunner, S., Stalder, B., \& Gangl, M. (2011). The impact of social origin and migration background on transition into post compulsory education and training. In M. M. Bergman, S. Hupka-Brunner, A. Keller, T. Meyer, \& B. E. Stalder (Eds.), Youth Transitions in Switzerland: Results from the TREE Panel Study (Vol. Volume 1, pp. 120156). Zürich: Seismo.

Sacchi, S., \& Meyer, T. (2016). Übergangslösungen beim Eintritt in die Schweizer Berufsbildung: Brückenschlag oder Sackgasse? Schweizerische Zeitschrift für Soziologie, 42(1), 9-39.

Scharenberg, K., Hupka-Brunner, S., Meyer, T., \& Bergman, M. M. (Eds.). (2016). Transitionen im Jugend- und jungen Erwachsenenalter: Ergebnisse der Schweizer Längsschnittstudie TREE (Vol. 2). Zürich: Seismo.

Schräder-Naef, R., \& Jörg-Fromm, R. (2005). Eine zweite Chance für Ungelernte? Auswirkungen des nachgeholten Lehrabschlusses. Zürich/Chur: Rüegger.

Schuchart, C., \& Weishaupt, H. (2004). Die prognostische Qualität der Übergangsempfehlungen der niedersächsischen Orientierungsstufe. Zeitschrift für Pädagogik, 6, 882-902.

Sheldon, G. (2008). Die Rolle der Berufsbildung in der Bekämpfung des Fachkräftemangels. Schlussbericht zu einem Auftrag des Bundesamtes für Berufsbildung und Technologie. Basel: Forschungsstelle für Arbeitsmarkt- und Industrieökonomik (FAI), Universität Basel.

SKBF (2014). Bildungsbericht Schweiz 2014. Aarau: Schweizerische Koordinationsstelle für Bildungsforschung.

Streckeisen, U., Hänzi, D., \& Hungerbühler, A. (2007). Fördern und Auslesen. Deutungsmuster von Lehrpersonen zu einem beruflichen Dilemma. Wiesbaden: VS Verlag für Sozialwissenschaften.

Trautwein, U., \& Baeriswyl, F. (2007). Wenn leistungsstarke Klassenkameraden ein Nachteil sind. Referenzgruppeneffekte bei Übertrittsentscheidungen. Zeitschrift für Pädagogische Psychologie, 2, 119-133.

Verachtert, P., van Damme, J., Onghena, P., \& Ghesquière, P. (2009). A seasonal perspective on school effectiveness: Evidence from a Flemish longitudinal study. School Effectiveness and School Improvement, 20(2), 215-233. doi:10.1080/09243450902883896

Wenzel, H. (2010). Chancengleichheit in der Schule - eine nicht abgegoltene Forderung. In H. H. Krüger, U. Rabe-Kleberg, R.-T. Kramer, \& J. Budde (Hrsg.), Bildungsungleichheiten revisited. Bildung und soziale Ungleichheiten vom Kindergarten bis zur Hochschule (Vol. 1, S.. 57-68). Wiesbaden: VS Verlag für Sozialwissenschaften. 
Open Access Dieses Kapitel wird unter der Creative Commons Namensnennung 4.0 International Lizenz (http://creativecommons.org/licenses/by/4.0/deed.de) veröffentlicht, welche die Nutzung, Vervielfältigung, Bearbeitung, Verbreitung und Wiedergabe in jeglichem Medium und Format erlaubt, sofern Sie den/die ursprünglichen Autor(en) und die Quelle ordnungsgemäß nennen, einen Link zur Creative Commons Lizenz beifügen und angeben, ob Änderungen vorgenommen wurden.

Die in diesem Kapitel enthaltenen Bilder und sonstiges Drittmaterial unterliegen ebenfalls der genannten Creative Commons Lizenz, sofern sich aus der Abbildungslegende nichts anderes ergibt. Sofern das betreffende Material nicht unter der genannten Creative Commons Lizenz steht und die betreffende Handlung nicht nach gesetzlichen Vorschriften erlaubt ist, ist für die oben aufgeführten Weiterverwendungen des Materials die Einwilligung des jeweiligen Rechteinhabers einzuholen.

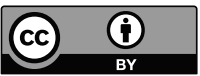




\section{Verzeichnis der Autorinnen und Autoren}

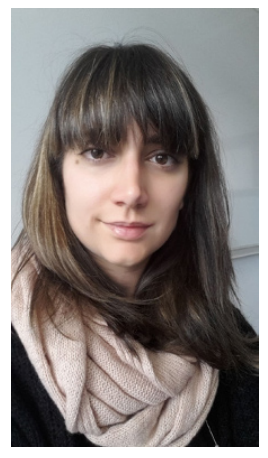

Benini, Sara, M Sc, Scuola universitaria professionale della Svizzera italiana (SUPSI), Dipartimento formazione e apprendimento (DFA), Locarno, sara.benini@supsi.ch Sara Benini ist Forscherin am DFA und ist am Departement für die nationalen und internationalen Beziehungen verantwortlich. Sie war zum Zeitpunkt der Erstellung dieses Artikels wissenschaftliche Mitarbeiterin am Zentrum Lernen und Sozialisation der PH FHNW, Solothurn. Sie war in der Konzipierung und Durchführung von Forschungsprojekten (insb. Interventionsstudien) tätig. Ihre Forschungsschwerpunkte sind schulische und kontextuelle Voraussetzungen bei der Förderung von Schülerinnen und Schülern im Bereich der obligatorischen Schule.

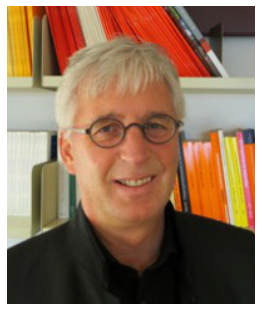

Bless, Gérard, Prof. Dr. phil., Heilpädagogisches Institut der Universität Freiburg i. Ue, gerard.bless@unifr.ch Gérard Bless ist Ordinarius für Sonderpädagogik an der Universität Freiburg. Gleichzeitig amtet er als Direktor des Heilpädagogischen Instituts und Leiter des Regionalen Schuldienstes. Fragestellungen zur schulischen Integration beziehungsweise Separation von Kindern mit sonderpädagogischem Förderbedarf, zur Klassenwiederholung, Bildungsstatistik, Lernbehinderung und zur Hörbehinderung bilden seine hauptsächlichen Forschungsschwerpunkte. 


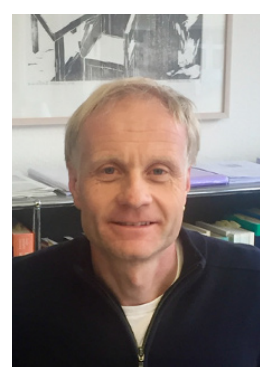

Düggeli, Albert, Prof. Dr., Pädagogische Hochschule FHNW, Basel, albert.dueggeli@fhnw.ch

Albert Düggeli ist Leiter der Professur für Entwicklungspsychologie und Pädagogik, Institut Sekundarstufe I \& II. Er forscht zu Resilienzentwicklung von Jugendlichen, die bildungsbiografisch riskante Transitionsprozesse durchlaufen. Ebenso gehören Fragen zur Nutzung von Durchlässigkeit in Bildungssystemen zu seinen Forschungsschwerpunkten, insbesondere mit Blick auf den Einfluss der familiären Herkunft beim Übergang von der Sekundarstufe I in die postobligatorische Ausbildung.

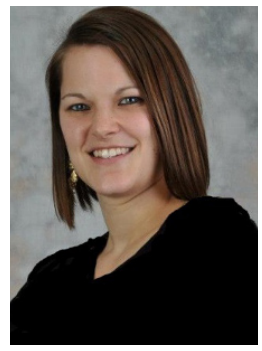

Fräulin, Jennifer C., M Sc, Pädagogische Hochschule FHNW, Solothurn, jennifer.fraeulin@fhnw.ch Jennifer C. Fräulin ist wissenschaftliche Mitarbeiterin am Zentrum Lernen und Sozialisation. Sie forscht und promoviert zum Thema Berufswahl von Jugendlichen und interessiert sich insbesondere für die kontextuellen Bedingungen der Berufsfindung zum Beispiel für die elterliche Unterstützung oder die Eltern-Lehrpersonen-Zusammenarbeit.

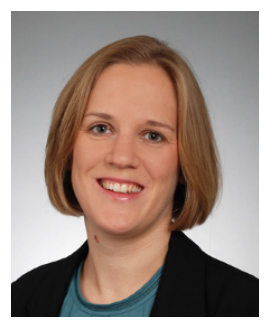

Frey, Simone, M Sc, Pädagogische Hochschule FHNW, Solothurn, simone.frey@fhnw.ch

Simone Frey ist wissenschaftliche Mitarbeiterin am Zentrum Lernen und Sozialisation. Sie betreut eine Längsschnittstudie zu Berufsbildungsverläufen. Sie forscht zu den Themen Laufbahnentwicklungen, Weiterbildungsentscheidungen und Vereinbarkeit von Familie und Beruf.

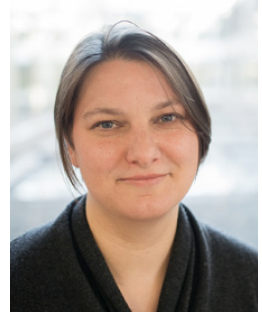

Hupka-Brunner, Sandra, Dr., Soziologisches Institut der Universität Bern, sandra.hupka@soz.unibe.ch Sandra Hupka-Brunner ist Ko-Leiterin des Projektes TREE (Transition von der Erstausbildung ins Erwerbsleben). Ihre Forschungsschwerpunkte sind Transitionen, soziale Ungleichheiten, Migration und Gender. 


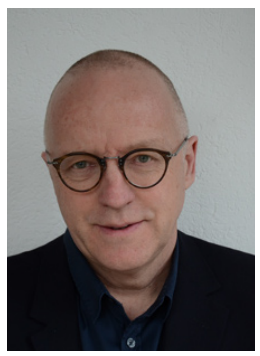

Moser, Urs, Prof. Dr., Institut für Bildungsevaluation, Assoziiertes Institut der Universität Zürich, urs.moser@ibe.uzh.ch Urs Moser ist Titularprofessor für das Gebiet Pädagogik mit Schwerpunkt empirische Bildungsforschung und Geschäftsleiter des Instituts für Bildungsevaluation. Seine Forschungsschwerpunkte umfassen Evaluationsforschung im Bildungsbereich, Methoden der Leistungsmessung, Schuleffektivitätsforschung und Schnittstellen des Bildungssystems.

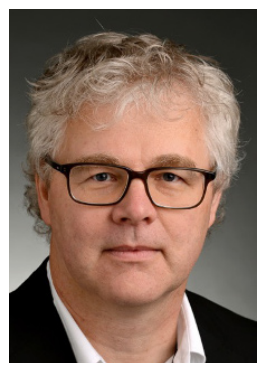

Nägele, Christof, Dr., Pädagogische Hochschule FHNW, Solothurn christof.naegele@fhnw.ch

Christof Nägele ist wissenschaftlicher Mitarbeiter und Projektleiter am Zentrum Lernen und Sozialisation. Er arbeitet zu folgenden Forschungsschwerpunkten: individuelle institutionelle und Bedingungen des Lernens, der schulischen und beruflichen Entwicklung unter besonderer Berücksichtigung sozialer Prozesse in der Gestaltung von Lehr- und Lernprozessen.

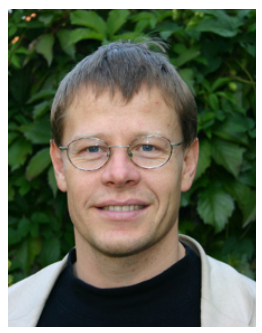

Neuenschwander, Markus P., Prof. Dr., Pädagogische Hochschule FHNW, Solothurn, markus.neuenschwander@ fhnw.ch

Markus Neuenschwander ist Professor für Pädagogische Psychologie, Leiter des Zentrums Lernen und Sozialisation und Mitglied der Institutsversammlung des Instituts für Bildungswissenschaften der Universität Basel. Seine Forschungsschwerpunkte sind Transitionsforschung, Sozialisation in Schule und Berufsbildung

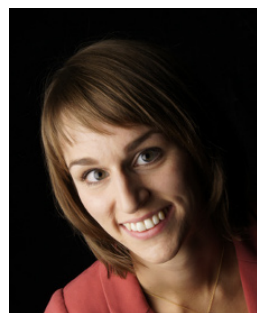

Niederbacher, Edith, Mag. phil., Pädagogische Hochschule FHNW, Solothurn, edith.niederbacher@fhnw.ch Edith Niederbacher ist wissenschaftliche Mitarbeiterin am Zentrum Lernen und Sozialisation. Sie forscht und promoviert zu den Themen Leistungsentwicklung und schulische Übergänge. Besonderen Fokus legt sie dabei auf die Rolle der familiären Unterstützung für Kinder und Jugendliche mit Migrationshintergrund. 


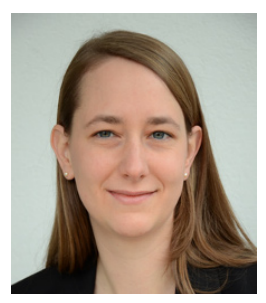

Oostlander, Jeannette, Dr. sc ETH, Institut für Bildungsevaluation, Assoziiertes Institut der Universität Zürich, jeannette.oostlander@ibe.uzh.ch Jeannette Oostlander ist wissenschaftliche Mitarbeiterin am Institut für Bildungsevaluation. Sie forscht zum Thema Schulleistungsmessung.

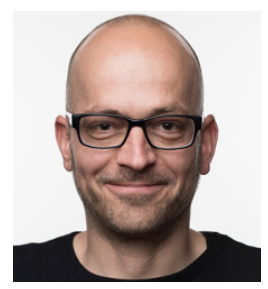

Rösselet, Stephan, lic. phil., Pädagogische Hochschule FHNW, Solothurn, stephan.roesselet@fhnw.ch / stephan. roesselet@bluewin.ch Stephan Rösselet war zum Zeitpunkt der Erstellung dieses Artikels wissenschaftlicher Mitarbeiter am Zentrum Lernen und Sozialisation. Er forscht zu den Themen Selbstkonzept und Bildungsdisparitäten.

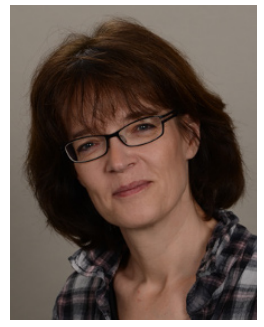

Stalder, Barbara E., Prof. Dr., Pädagogische Hochschule Bern, barbara.stalder@phbern.ch Barbara E. Stalder leitet den Bereich Erziehungs- und Sozialwissenschaften am Institut Sekundarstufe II der Pädagogischen Hochschule Bern und ist assoziierte Professorin an der Universität Neuenburg. Ihre Forschungsinteressen sind Laufbahnentwicklung, Lehrvertragsauflösungen, Ausbildungs- und Berufserfolg

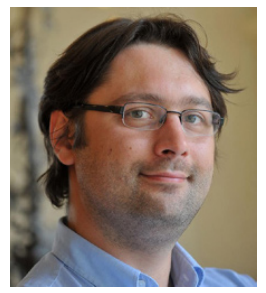

Tomasik, Martin, PD Dr., Institut für Bildungsevaluation, Assoziiertes Institut der Universität Zürich, martin. tomasik@ibe.uzh.ch

Martin Tomasik ist Oberassistent am Institut für Bildungsevaluation. Seine Forschungsschwerpunkte umfassen die Entwicklung im Jugend- und Erwachsenenalter, die Rolle von sozialen Kontexten sowie Bildungsübergänge im Jugendalter und jungem Erwachsenenalter. 